\title{
Using neighboring-group participation for acyclic stereocontrol in diastereoselective substitution reactions of acetals
}

\author{
Amanda Ramdular and K. A. Woerpel
}

Supplemental Information

Department of Chemistry, New York University, 100 Washington Square East, New York, NY 10003 (USA)

\section{Table of Contents}

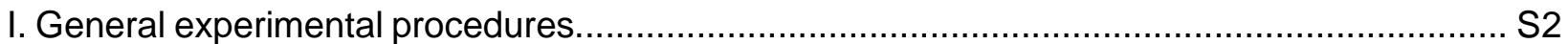

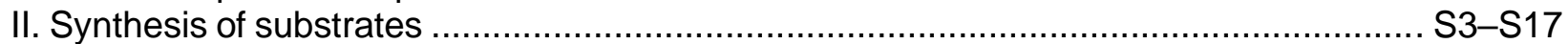



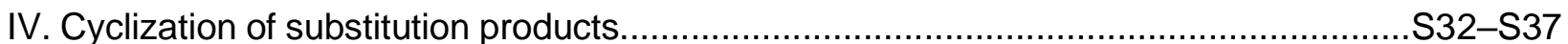

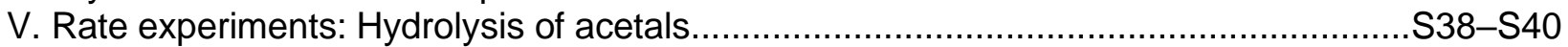



A. DPFGSE-nOe experiments........................................................ S41-S48

B. X-ray crystallography of 8c......................................................... S49-S54

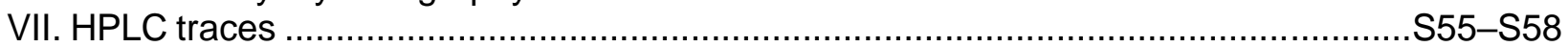

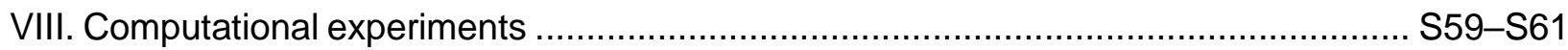



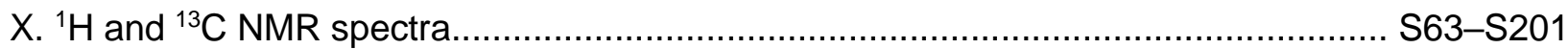

A. NMR spectra of purified compounds..................................................... S63-S191

B. NMR spectra of unpurified reaction mixtures ..................................... S192-S201 
I. General experimental procedures. ${ }^{1} \mathrm{H}$ and ${ }^{13} \mathrm{C}$ NMR spectra were obtained at room temperature using a Bruker AV-400 spectrometer at 400 and $100 \mathrm{MHz}$, or a Bruker AV-600 spectrometer at 600 and $150 \mathrm{MHz}$, as indicated. nOe data were obtained at room temperature using a Bruker AV-600 spectrometer at $600 \mathrm{MHz}$. The data are reported as follows: chemical shift in ppm referenced to residual solvent ( ${ }^{1} \mathrm{H} \mathrm{NMR}: \mathrm{CDCl}_{3} \delta 7.26 ;{ }^{13} \mathrm{C} \mathrm{NMR} \mathrm{CDCl}_{3} 77.16$;), multiplicity ( $\mathrm{br}=$ broad, $\mathrm{s}=$ singlet, $\mathrm{d}=$ doublet, $\mathrm{t}=$ triplet, $\mathrm{q}=$ quartet, $\mathrm{m}=$ multiplet), coupling constants $(\mathrm{Hz})$, and integration. Peak assignments were determined using HSQC, DEPT 135, HMBC, and COSY experiments. Infrared (IR) spectra were recorded using a Thermo Nicolet AVATAR Fourier Transform IR spectrometer using attenuated total reflectance (ATR). High resolution mass spectra (HRMS) were acquired on an Agilent 6224 Accurate-Mass time-of-flight LC/TOF spectrometer with atmospheric pressure chemical ionization (APCI) or electrospray (ESI) ionization source and were obtained by peak matching. Melting points were reported uncorrected. Analytical thin layer chromatography was performed on silica get $60 \AA$ F254 plates. Liquid chromatography was performed using forced flow (flash chromatography) of the indicated solvent mixture on Silicycle silica gel $\left(\mathrm{SiO}_{2}\right) 60$ (230-400 mesh). All reactions were performed under an atmosphere of nitrogen in glassware that had been flame-dried under vacuum unless otherwise stated. Non-deuterated solvents were purified via the Pure Solv-MD Standard Design Solvent Purification System before use. Aqueous solutions were prepared from nanopure water with a resistivity over $18 \mathrm{M} \Omega-\mathrm{cm}$. Unless otherwise stated, all reagents and substrates were commercially available. 


\section{Synthesis of substrates}

Scheme S1. General substrate synthesis.

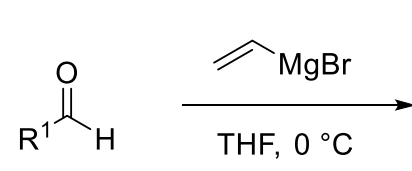

S1<smiles>[R]C(O)C=C</smiles>

S2

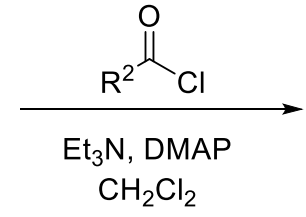

$\mathrm{R}$<smiles>[R]C(=O)OC([R])C(=O)O</smiles>

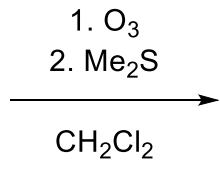

$\mathrm{CH}_{2} \mathrm{Cl}_{2}$<smiles>[R]C(=O)OC([R])C([R])=O</smiles><smiles>[R]C(=O)OC([R])C(OCC)OCCO</smiles>

S5

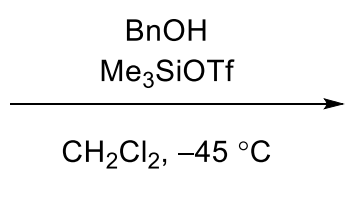<smiles>[R]C(=O)OC([R])C(O)OCc1ccccc1</smiles>

s6

General procedure for addition of vinylmagnesium bromide to aldehydes. Allylic alcohols were prepared from a procedure adapted from Cossy et. al. ${ }^{1}$ To a cooled $\left(0{ }^{\circ} \mathrm{C}\right)$ solution of aldehyde (1 equiv) in THF (1 M) was added vinylmagnesium bromide (1.0 M in $\mathrm{Et}_{2} \mathrm{O}, 1.5$ equiv). The reaction mixture was then warmed to room temperature. After $30 \mathrm{~min}, 5 \% \mathrm{HCl}$ aqueous solution $(20 \mathrm{~mL}$ per $\mathrm{mmol})$ was added, and the aqueous layer was extracted with EtOAc $(2 \times 20$ $\mathrm{mL}$ per mmol). The organic layers were combined, washed with brine (20 $\mathrm{mL}$ per mmol), dried over $\mathrm{Na}_{2} \mathrm{SO}_{4}$, filtered through cotton, and concentrated in vacuo to provide a yellow oil. The oil was subjected to flash column chromatography (10:90 EtOAc:hexanes) to provide the pure allylic alcohol.<smiles>CCCCC</smiles>

S7

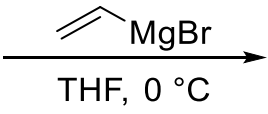

$\mathrm{THF}, 0^{\circ} \mathrm{C}$<smiles>C=C[15CH]C(O)C=C</smiles>

S8

Dec-1-en-3-ol (S8). Allylic alcohol S8 was prepared according to the general procedure for addition of vinylmagnesium bromide to aldehydes. Octanal $(6.23 \mathrm{~g}, 40.0 \mathrm{mmol})$ in THF $(40 \mathrm{~mL})$ was mixed with vinylmagnesium bromide $\left(1.0 \mathrm{M}\right.$ in $\left.\mathrm{Et}_{2} \mathrm{O}, 60 \mathrm{~mL}\right)$ to provide allylic alcohol $\mathbf{S 8}$ as a colorless oil $(6.01 \mathrm{~g}, 96 \%)$. The spectral data obtained are consistent with those reported: ${ }^{2}$

${ }^{1} \mathrm{H}$ NMR $\left(400 \mathrm{MHz}, \mathrm{CDCl}_{3}\right) \delta 5.88$ (ddd, $\left.J=17.2,10.6,6.2,1 \mathrm{H}\right), 5.23(\mathrm{dt}, J=18.1,1.5,1 \mathrm{H}), 5.11$ $(\mathrm{dt}, J=10.5,1.5,1 \mathrm{H}), 4.11(\mathrm{q}, J=6.2,1 \mathrm{H}), 1.51-1.56(\mathrm{~m}, 3 \mathrm{H}), 1.28-1.29(\mathrm{br} \mathrm{s}, 10 \mathrm{H}), 0.89(\mathrm{t}, J=$ 6.6, 3H);

${ }^{13} \mathrm{C}$ NMR (100 MHz, CDCl 3$) \delta 141.9(\mathrm{CH}), 115.1\left(\mathrm{CH}_{2}\right), 73.9(\mathrm{CH}), 37.7\left(\mathrm{CH}_{2}\right), 32.4\left(\mathrm{CH}_{2}\right), 30.1$ $\left(\mathrm{CH}_{2}\right), 29.8\left(\mathrm{CH}_{2}\right), 25.9\left(\mathrm{CH}_{2}\right), 23.2\left(\mathrm{CH}_{2}\right), 14.7\left(\mathrm{CH}_{3}\right)$;

IR (ATR) 3374, 2926, 1456, 1065, $919 \mathrm{~cm}^{-1}$.<smiles>O=CC1CCCCC1</smiles>

S9

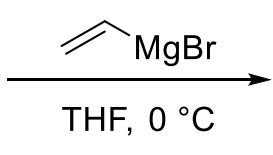

$\mathrm{THF}, 0^{\circ} \mathrm{C}$<smiles>C=CC(O)C1CCCCC1</smiles>

S10

1-Cyclohexylprop-2-en-1-ol (S10). Allylic alcohol S10 was prepared according to the general procedure for vinylmagnesium bromide addition to aldehydes. Cyclohexanecarboxaldehyde (1.62 



provide allylic alcohol $\mathbf{S 1 0}$ as a colorless oil $(2.00 \mathrm{~g}, 99 \%)$. The spectral data obtained are consistent with those reported: ${ }^{1}$

${ }^{1} \mathrm{H}$ NMR $\left(400 \mathrm{MHz}, \mathrm{CDCl}_{3}\right) \delta 5.88$ (ddd, $\left.J=16.9,10.3,6.7,1 \mathrm{H}\right), 2.21$ (dt, $\left.J=17.6,1.5,1 \mathrm{H}\right), 5.15$ $(\mathrm{dt}, J=10.2,1.5,1 \mathrm{H}), 3.86(\mathrm{t}, J=6.4,1 \mathrm{H}), 3.45(\mathrm{~d}, J=6.6,1 \mathrm{H}), 1.73-1.78(\mathrm{~m}, 3 \mathrm{H}), 1.67-1.70$ $(\mathrm{m}, 2 \mathrm{H}), 1.41-1.69(\mathrm{~m}, 3 \mathrm{H}), 0.96-1.68(\mathrm{~m}, 3 \mathrm{H})$;

${ }^{13} \mathrm{C}$ NMR $\left(100 \mathrm{MHz}, \mathrm{CDCl}_{3}\right) \delta 139.8(\mathrm{CH}), 115.5\left(\mathrm{CH}_{2}\right), 77.8(\mathrm{CH}), 43.5(\mathrm{CH}), 28.7\left(\mathrm{CH}_{2}\right), 28.4$ $\left(\mathrm{CH}_{2}\right), 26.5\left(\mathrm{CH}_{2}\right), 26.1\left(\mathrm{CH}_{2}\right), 26.0\left(\mathrm{CH}_{2}\right)$;

IR (ATR) 3578, 2987, 1264, 895, $804 \mathrm{~cm}^{-1}$;

HRMS (APCl) m/z: [[M + H] $\left.-\mathrm{H}_{2} \mathrm{O}\right]^{+}$Calcd for $\mathrm{C}_{9} \mathrm{H}_{15}$ 123.1168; Found 123.1170.

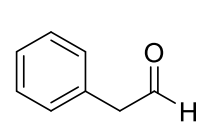

S11

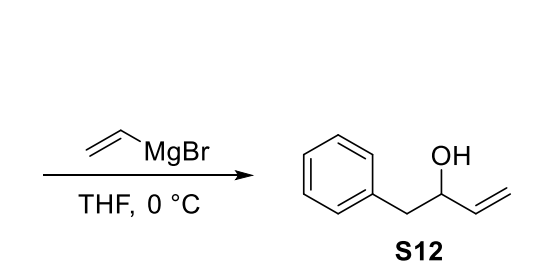

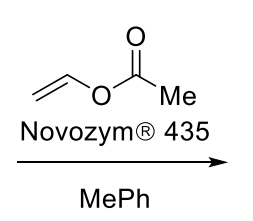

MePh

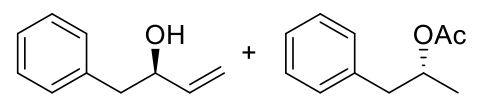

$(R)-\mathbf{S} 12$
(S)-S13

$(\boldsymbol{R})$-1-Phenylbut-3-en-2-ol ((R)-S12). Allylic alcohol $\mathbf{S 1 2}$ was prepared according to the general procedure for vinylmagnesium bromide addition to aldehydes. Phenylacetaldehyde $(2.4 \mathrm{~g}, 20$ $\mathrm{mmol})$ in THF $(20 \mathrm{~mL})$ was mixed with vinylmagnesium bromide $\left(1.0 \mathrm{M}\right.$ in $\left.\mathrm{Et}_{2} \mathrm{O}, 30 \mathrm{~mL}\right)$ to provide allylic alcohol $\mathbf{S 1 2}$ as a yellow oil $(2.96 \mathrm{~g}, 99 \%)$. The racemic mixture of allylic alcohol $\mathbf{S 1 2}$ was then subjected to kinetic resolution to obtain the $(R)$ enantiomer.

This procedure was adapted from that reported by Han et. al. ${ }^{3}$ To a solution of allylic alcohol $\mathbf{S 1 2}$ $(1.12 \mathrm{~g}, 10.0 \mathrm{mmol})$ in toluene $(10 \mathrm{~mL}, 0.10 \mathrm{M})$ was added vinyl acetate $(0.46 \mathrm{~mL}, 5.0 \mathrm{mmol})$ and Novozym® $435(200 \mathrm{mg})$. After $40 \mathrm{~h}$, the reaction mixture was filtered over a pad of silica and concentrated in vacuo to provide a yellow oil. The oil was purified by flash column chromatography (10:90 EtOAc:hexanes) to obtain the enantiomerically enriched allylic acetate (S)-S13 (0.594 g, $31 \%$, e.r. = 91:9) and allylic alcohol $(R)-\mathbf{S} 12(0.597 \mathrm{~g}, 40 \%$, e.r. $=91: 9)$, as determined by HPLC (Chiralcel OD-H, 10:90 $\mathrm{PrOH}$ :hexane, $220 \mathrm{~nm}$, flow rate $1 \mathrm{~mL} / \mathrm{min}$ ). The enriched allylic alcohol $(R)$-S12 and remaining racemic mixture were combined $(0.897 \mathrm{~g}, 6.00 \mathrm{mmol})$ and subjected to kinetic resolution to provide the allylic alcohol $(R)$-S12 as a single enantiomer $(0.65 \mathrm{~g}, 67 \%$, e.r. $=98: 2)$. The spectral data obtained are consistent with those reported: ${ }^{3}$

${ }^{1} \mathrm{H} \mathrm{NMR}\left(400 \mathrm{MHz}, \mathrm{CDCl}_{3}\right) \delta 7.30(\mathrm{~m}, 2 \mathrm{H}), 7.22-7.24(\mathrm{~m}, 3 \mathrm{H}), 5.92$ (ddd, $\left.J=16.5,10.5,5.9,1 \mathrm{H}\right)$, $5.22(\mathrm{dt}, J=17.2,1.5,1 \mathrm{H}), 5.12(\mathrm{dt}, J=10.6,1.5,1 \mathrm{H}), 4.33(\mathrm{q}, J=5.9,1 \mathrm{H}), 2.86(\mathrm{dd}, J=14.3$, $4.9,1 \mathrm{H}), 2.78(\mathrm{dd}, J=14.7,7.9,1 \mathrm{H}), 1.77(\mathrm{~s}, 1 \mathrm{H})$;

${ }^{13} \mathrm{C}$ NMR $\left(100 \mathrm{MHz}, \mathrm{CDCl}_{3}\right) \delta 140.3(\mathrm{C}), 137.8(\mathrm{CH}), 129.7(\mathrm{CH}), 128.6(\mathrm{CH}), 126.7(\mathrm{CH}), 115.0$ $\left(\mathrm{CH}_{2}\right), 73.7(\mathrm{CH}), 43.9\left(\mathrm{CH}_{2}\right)$;

IR (ATR) 3368, 2919, 1116, 921, $744 \mathrm{~cm}^{-1}$;

HRMS (APCl) m/z: [[M + H] $\left.-\mathrm{H}_{2} \mathrm{O}\right]^{+}$Calcd for $\mathrm{C}_{10} \mathrm{H}_{11}$ 131.0855; Found 131.0863.
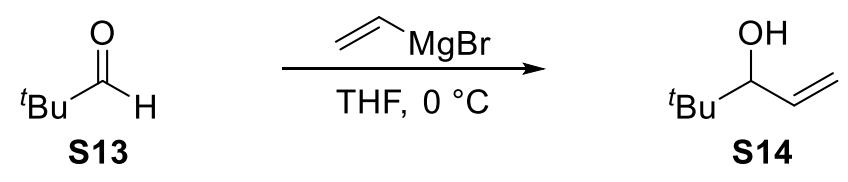

4,4-Dimethylpent-1-en-3-ol (S14). Allylic alcohol S14 was prepared according to the general procedure for vinylmagnesium bromide addition to aldehydes. Pivaldehyde $(2.17 \mathrm{~g}, 20.0 \mathrm{mmol})$ in THF $(20 \mathrm{~mL})$ was mixed with vinylmagnesium bromide $\left(1.0 \mathrm{M} \mathrm{in} \mathrm{Et}_{2} \mathrm{O}, 30 \mathrm{~mL}\right)$ to provide allylic alcohol S14 as a colorless oil (1.44 g, 63\%). The spectral data obtained are consistent with those reported: ${ }^{1}$

${ }^{1} \mathrm{H}$ NMR $\left(400 \mathrm{MHz}, \mathrm{CDCl}_{3}\right) \delta 5.92$ (ddd, $\left.J=16.5,10.5,6.6,1 \mathrm{H}\right), 5.24(\mathrm{~d}, J=17.6,1 \mathrm{H}), 5.18(\mathrm{~d}, J$ $=10.1,1 \mathrm{H}), 3.76(\mathrm{~d}, J=6.4,1 \mathrm{H}), 0.93(\mathrm{~s}, 10 \mathrm{H})$; 



IR (ATR) 3371, 2957, 2922, 1118, $1047 \mathrm{~cm}^{-1}$.<smiles>C=CC(O)c1ccc(C)cc1</smiles>

1-(p-Tolyl)prop-2-en-1-ol (S16). Allylic alcohol S16 was prepared according to the general procedure for vinylmagnesium bromide addition to aldehydes. Tolualdehyde $(12 \mathrm{~g}, 0.10 \mathrm{~mol})$ in THF $(0.2 \mathrm{~L})$ was mixed with vinylmagnesium bromide $\left(1.0 \mathrm{M}\right.$ in $\left.\mathrm{Et}_{2} \mathrm{O}, 0.15 \mathrm{~L}\right)$ to provide allylic alcohol $\mathbf{S 1 6}$ as a colorless oil (14 g, 98\%). The spectral data obtained are consistent with those reported:4

${ }^{1} \mathrm{H}$ NMR $\left(400 \mathrm{MHz}, \mathrm{CDCl}_{3}\right) \delta 7.22$ (d, $\left.J=7.8,2 \mathrm{H}\right), 7.13$ (d, $J=7.8,2 \mathrm{H}$ ), 5.99 (ddd, $J=16.9,10.4$, $6.8,1 \mathrm{H}), 5.29(\mathrm{dt}, J=17.7,1.5,1 \mathrm{H}), 5.14(\mathrm{dt}, J=10.6,1.5,1 \mathrm{H}), 5.10(\mathrm{br} \mathrm{s}, 1 \mathrm{H}), 2.32(\mathrm{~s}, 3 \mathrm{H}), 2.30$ (br s, $1 \mathrm{H})$;

${ }^{13} \mathrm{C}$ NMR (100 MHz, $\left.\mathrm{CDCl}_{3}\right)$ ס $140.5(\mathrm{C}), 139.8(\mathrm{C}), 137.4(\mathrm{CH}), 129.3(\mathrm{CH}), 126.4(\mathrm{CH}), 114.9$ $\left(\mathrm{CH}_{2}\right), 75.2(\mathrm{CH}), 21.2\left(\mathrm{CH}_{3}\right)$;

IR (ATR) 3343, 2921, 986, 814, $772 \mathrm{~cm}^{-1}$.<smiles>O=Cc1cccc2ccccc12</smiles>

S17

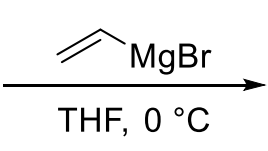<smiles>C=CC(O)c1cccc2ccccc12</smiles>

S18

1-(Naphthalen-1-yl)prop-2-en-1-ol (S18). Allylic alcohol S18 was prepared according to the general procedure for vinylmagnesium bromide addition to aldehydes. 1-Naphthaldehyde $(0.311$ $\mathrm{g}, 2.00 \mathrm{mmol})$ in THF (4 mL) was mixed with vinylmagnesium bromide $\left(1 \mathrm{M}\right.$ in $\left.\mathrm{Et}_{2} \mathrm{O}, 3 \mathrm{~mL}\right)$ to provide allylic alcohol $\mathbf{S 1 8}$ as a yellow oil $(0.365 \mathrm{~g}, 99 \%)$. The spectral data obtained are consistent with those reported: 5

${ }^{1} \mathrm{H}$ NMR $\left(400 \mathrm{MHz}, \mathrm{CDCl}_{3}\right) \delta 8.21(\mathrm{~d}, J=8.1,1 \mathrm{H}), 7.89(\mathrm{dd}, J=8.4,1.7,1 \mathrm{H}), 7.82(\mathrm{~d}, J=8.2$, $1 \mathrm{H}), 7.64(\mathrm{~d}, J=7.1,1 \mathrm{H}), 7.47-7.57(\mathrm{~m}, 3 \mathrm{H}), 6.27(\mathrm{ddd}, J=15.8,10.5,5.3,1 \mathrm{H}), 5.96(\mathrm{t}, J=1.5$, $1 \mathrm{H}), 5.47(\mathrm{dt}, J=17.4,1.5,1 \mathrm{H}), 5.30(\mathrm{dt}, J=10.8,1.5,1 \mathrm{H}), 2.09(\mathrm{~d}, J=4.4,1 \mathrm{H})$;

${ }^{13} \mathrm{C}$ NMR (100 MHz, $\mathrm{CDCl}_{3}$ ) $\delta 139.8(\mathrm{C}), 138.2(\mathrm{C}), 134.1(\mathrm{C}), 130.8(\mathrm{CH}), 128.9(\mathrm{CH}), 128.7$ $(\mathrm{CH}), 126.3(\mathrm{CH}), 125.8(\mathrm{CH}), 125.6(\mathrm{CH}), 124.1(\mathrm{CH}), 123.9(\mathrm{CH}), 115.8\left(\mathrm{CH}_{2}\right), 72.5(\mathrm{CH})$; IR (ATR) 3456, 2981, 1042, 991, $783 \mathrm{~cm}^{-1}$.

General procedure for esterification of allylic alcohols. Allylic esters were prepared from a procedure adapted from Wu et. al. ${ }^{4}$ To a cooled solution $\left(0^{\circ} \mathrm{C}\right)$ of the allylic alcohol in $\mathrm{CH}_{2} \mathrm{Cl}_{2}(1$ $\mathrm{M})$ was added $\mathrm{Et}_{3} \mathrm{~N}$ (2 equiv) and dimethylaminopyridine (0.1 equiv). After $30 \mathrm{~min}$, the corresponding acyl chloride ( 2 equiv) was added dropwise over $5 \mathrm{~min}$. After $8 \mathrm{~h}$, a $5 \% \mathrm{HCl}$ solution (20 $\mathrm{mL}$ per $10 \mathrm{mmol})$ was added, and the aqueous layer was extracted with $\mathrm{CH}_{2} \mathrm{Cl}_{2}(2 \times 30 \mathrm{~mL}$ per $10 \mathrm{mmol})$. The organic layers were combined, washed with brine $(20 \mathrm{~mL}$ per $10 \mathrm{mmol})$, dried over sodium sulfate, filtered through cotton, and concentrated in vacuo to provide a yellow oil. The oil was purified by column chromatography (5:95 EtOAc:hexanes) to provide the pure ester. 
<smiles>C=CC(O)CC</smiles>

S8

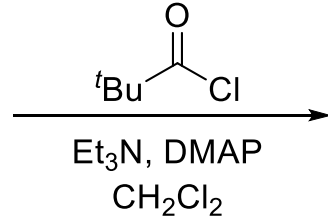

$\mathrm{CH}_{2} \mathrm{Cl}_{2}$<smiles>C=CC(CCCC)OC(=O)Br</smiles>

$\mathbf{S 1 9}$

Dec-1-en-3-yl pivaloate (S19). Following the general procedure for esterification, allylic alcohol S8 $(2.04 \mathrm{~g}, 13.0 \mathrm{mmol})$ in $\mathrm{CH}_{2} \mathrm{Cl}_{2}(13 \mathrm{~mL})$ was mixed with $\mathrm{Et}_{3} \mathrm{~N}$ (3.61 mL, $\left.26.0 \mathrm{mmol}\right)$, dimethylaminopyridine $(0.159 \mathrm{~g}, 1.30 \mathrm{mmol})$, and trimethylacetyl chloride $(2.21 \mathrm{~mL}, 26.0 \mathrm{mmol})$ to give ester $\mathbf{S 1 9}$ a colorless oil (3.15 g, 99\%):

${ }^{1} \mathrm{H}$ NMR $\left(400 \mathrm{MHz}, \mathrm{CDCl}_{3}\right) \delta 5.78$ (ddd, $\left.J=17.4,10.4,6.1,1 \mathrm{H}\right), 5.19-5.24(\mathrm{~m}, 2 \mathrm{H}), 5.13(\mathrm{dt}, J=$ 10.7, 1.5, 1H), 1.27 (br s, 6H), $1.23(\mathrm{~s}, 9 \mathrm{H}), 1.19-1.21(\mathrm{~m}, 6 \mathrm{H}), 0.89$ (t, $J=6.4,3 \mathrm{H})$;

${ }^{13} \mathrm{C}$ NMR $\left(100 \mathrm{MHz}, \mathrm{CDCl}_{3}\right) \delta 178.3(\mathrm{C}), 137.5(\mathrm{CH}), 116.4\left(\mathrm{CH}_{2}\right), 74.8(\mathrm{CH}), 39.4\left(\mathrm{CH}_{2}\right), 34.8$ (C), $32.3\left(\mathrm{CH}_{2}\right), 29.9\left(\mathrm{CH}_{2}\right), 27.79\left(\mathrm{CH}_{2}\right), 27.75\left(\mathrm{CH}_{3}\right), 25.6\left(\mathrm{CH}_{2}\right), 23.2\left(\mathrm{CH}_{2}\right), 14.7\left(\mathrm{CH}_{3}\right)$; IR (ATR) 2929, 1728, 1155, 1002, $737 \mathrm{~cm}^{-1}$.<smiles>C=CC(O)C1CCCCC1</smiles>

S10



$\mathrm{CH}_{2} \mathrm{Cl}_{2}$<smiles>C=CC(OC(=O)Br)C1CCCCC1=O</smiles>

1-Cyclohexylallyl pivaloate (S20). Following the general procedure for esterification, allylic alcohol S10 (2.02 g, $15.0 \mathrm{mmol})$ in $\mathrm{CH}_{2} \mathrm{Cl}_{2}(15 \mathrm{~mL})$ was mixed with $\mathrm{Et}_{3} \mathrm{~N}(3.05 \mathrm{~mL}, 22.0 \mathrm{mmol})$, dimethylaminopyridine $(0.134 \mathrm{~g}, 1.50 \mathrm{mmol})$, and trimethylacetyl chloride $(1.87 \mathrm{~mL}, 22.0 \mathrm{mmol})$ to give ester $\mathbf{S} 20$ as a colorless oil $(3.3 \mathrm{~g}, 97 \%)$. The spectral data obtained are consistent with those reported: 4

${ }^{1} \mathrm{H}$ NMR $\left(400 \mathrm{MHz}, \mathrm{CDCl}_{3}\right) \delta 5.76$ (ddd, $\left.J=17.1,10.4,6.4,1 \mathrm{H}\right), 5.19(\mathrm{dt}, J=13.4,1.4,1 \mathrm{H}), 5.16$ $(\mathrm{dt}, J=6.7,1.4,1 \mathrm{H}), 5.03(\mathrm{t}, J=6.4,1 \mathrm{H}), 1.66-1.77(\mathrm{~m}, 6 \mathrm{H}), 1.51-1.60(\mathrm{~m}, 2 \mathrm{H}), 1.22(\mathrm{~s}, 9 \mathrm{H})$, $0.97-1.06(\mathrm{~m}, 3 \mathrm{H})$;

${ }^{13} \mathrm{C}$ NMR $\left(100 \mathrm{MHz}, \mathrm{CDCl}_{3}\right) \delta 177.8(\mathrm{C}), 135.4(\mathrm{CH}), 116.8\left(\mathrm{CH}_{2}\right), 78.2(\mathrm{CH}), 41.7(\mathrm{CH}), 38.9$ $\left(\mathrm{CH}_{2}\right), 28.8\left(\mathrm{CH}_{2}\right), 28.1\left(\mathrm{CH}_{2}\right), 27.4\left(\mathrm{CH}_{2}\right), 26.5\left(\mathrm{CH}_{2}\right), 26.0(\mathrm{C}), 25.7\left(\mathrm{CH}_{3}\right)$;

IR (ATR) 2929, 2854, 1728, 1155, $940 \mathrm{~cm}^{-1}$;

HRMS (APCl) m/z: [[M + H] $\left.-\mathrm{C}_{5} \mathrm{H}_{10} \mathrm{O}_{2}\right]^{+}$Calcd for $\mathrm{C}_{9} \mathrm{H}_{15}$ 123.1168; Found 123.1170.<smiles>C=C[C@H](O)Cc1ccccc1</smiles>

(R)-S12

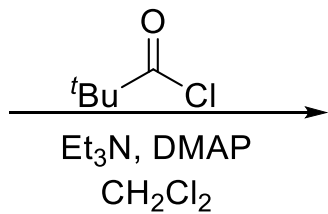

$\mathrm{CH}_{2} \mathrm{Cl}_{2}$

$(R)$-1-Phenylbut-3-en-2-yl pivaloate $((R)-S 21)$. Following the general procedure for esterification, allylic alcohol $(R)-\mathrm{S} 12(0.65 \mathrm{~g}, 4.4 \mathrm{mmol})$ in $\mathrm{CH}_{2} \mathrm{Cl}_{2}(9 \mathrm{~mL})$ was mixed with $\mathrm{Et}_{3} \mathrm{~N}(1.2$ $\mathrm{mL}, 8.8 \mathrm{mmol})$, dimethylaminopyridine $(0.054 \mathrm{~g}, 0.44 \mathrm{mmol})$, and trimethylacetyl chloride $(1.1 \mathrm{~mL}$, $8.8 \mathrm{mmol})$ to give ester $(R)$-S21 as a colorless oil $(0.95 \mathrm{~g}, 93 \%)$ :

${ }^{1} \mathrm{H}$ NMR $\left(400 \mathrm{MHz}, \mathrm{CDCl}_{3}\right) \delta 7.24(\mathrm{~d}, J=6.9,2 \mathrm{H}), 7.19(\mathrm{~d}, J=6.1,3 \mathrm{H}), 5.82$ (ddd, $J=16.7,10.4$, $5.8,1 \mathrm{H}), 5.45(\mathrm{q}, J=6.4,1 \mathrm{H}), 5.21(\mathrm{~d}, J=17.1,1 \mathrm{H}), 5.13(\mathrm{~d}, J=10.7,1 \mathrm{H}), 2.91(\mathrm{~d}, J=6.9,2 \mathrm{H})$, $1.11(\mathrm{~s}, 9 \mathrm{H})$;

${ }^{13} \mathrm{C}$ NMR (100 MHz, $\left.\mathrm{CDCl}_{3}\right) \delta 177.6(\mathrm{C}), 137.1(\mathrm{C}), 136.2(\mathrm{CH}), 129.7(\mathrm{CH}), 128.3(\mathrm{CH}), 126.6$ $(\mathrm{CH}), 116.4\left(\mathrm{CH}_{2}\right), 74.6(\mathrm{CH}), 41.1\left(\mathrm{CH}_{2}\right), 38.9(\mathrm{C}), 27.2\left(\mathrm{CH}_{3}\right)$; 
IR (ATR) 2974, 1728, 1147, 1000, $973 \mathrm{~cm}^{-1}$;

HRMS (APCl) m/z: [[M + H] $\left.-\mathrm{C}_{5} \mathrm{H}_{10} \mathrm{O}_{2}\right]^{+}$Calcd for $\mathrm{C}_{10} \mathrm{H}_{11}$ 131.0855; Found 131.0858 .<smiles>C=CC(O)Cc1ccccc1</smiles>

S12

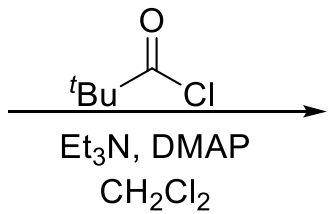

$\mathrm{CH}_{2} \mathrm{Cl}_{2}$<smiles>C=CC(Cc1ccccc1)OC(=O)C(C)(C)C</smiles>

S21

1-Phenylbut-3-en-2-yl pivaloate (S21). Following the general procedure for esterification, allylic alcohol $\mathrm{S} 12(0.52 \mathrm{~g}, 3.5 \mathrm{mmol})$ in $\mathrm{CH}_{2} \mathrm{Cl}_{2}(7 \mathrm{~mL})$ was mixed with $\mathrm{Et}_{3} \mathrm{~N}(0.9 \mathrm{~mL}, 7 \mathrm{mmol})$, dimethylaminopyridine $(0.043 \mathrm{~g}, 0.35 \mathrm{mmol})$, and trimethylacetyl chloride $(0.86 \mathrm{~mL}, 7.0 \mathrm{mmol})$ to give ester (rac)-S21 as a colorless oil (0.804 g, 99\%). The spectral data obtained are consistent with those reported above for the enantiomerically enriched compound.<smiles>C=CC(O)c1ccccc1</smiles>



.<smiles>C=CC(Br)(Br)OC(=O)CC(C)C</smiles>

S22

4,4-Dimethylpent-1-en-3-yl pivaloate (S22). Following the general procedure for esterification, allylic alcohol $\mathbf{S 1 4}(0.72 \mathrm{~g}, 6.3 \mathrm{mmol})$ in $\mathrm{CH}_{2} \mathrm{Cl}_{2}(6.5 \mathrm{~mL})$ was mixed with $\mathrm{Et}_{3} \mathrm{~N}(1.76 \mathrm{~mL}, 12.6$ $\mathrm{mmol})$, dimethylaminopyridine $(0.077 \mathrm{~g}, 0.63 \mathrm{mmol})$, and trimethylacetyl chloride $(1.54 \mathrm{~mL}, 12.6$ $\mathrm{mmol}$ ) to give ester $\mathbf{S 2 2}$ as a colorless oil $(1.15 \mathrm{~g}, 92 \%)$. The spectral data obtained are consistent with those reported:4

${ }^{1} \mathrm{H}$ NMR (400 MHz, $\mathrm{CDCl}_{3}$ ) $\delta 5.80$ (ddd, $\left.J=16.7,10.2,6.6,1 \mathrm{H}\right), 5.22(\mathrm{~d}, J=4.9,1 \mathrm{H}), 5.19(\mathrm{~d}, J$ $=1.1,1 \mathrm{H}), 4.95(\mathrm{~d}, J=6.4,1 \mathrm{H}), 1.24(\mathrm{~s}, 9 \mathrm{H}), 0.94(\mathrm{~s}, 9 \mathrm{H})$;

${ }^{13} \mathrm{C}$ NMR (100 MHz, CDCl $)$ $) 177.6(\mathrm{C}), 133.9(\mathrm{CH}), 117.8\left(\mathrm{CH}_{2}\right), 81.4(\mathrm{CH}), 39.2(\mathrm{C}), 34.5(\mathrm{C})$, $27.4\left(\mathrm{CH}_{3}\right), 25.9\left(\mathrm{CH}_{3}\right)$;

IR (ATR) 2973, 1729, 1480, 1199, $1042 \mathrm{~cm}^{-1}$.<smiles>C=CC(O)c1ccc(C)cc1</smiles>

S16

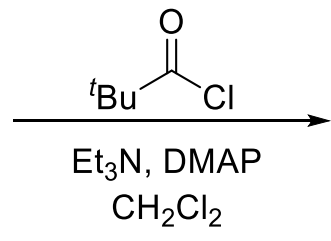

$\mathrm{CH}_{2} \mathrm{Cl}_{2}$<smiles>C=CC(OC(=O)CC(C)(C)C)c1ccc(C)cc1</smiles>

S23

1-(p-Tolyl)allyl pivaloate (S23). Following the general procedure for esterification, allylic alcohol S16 (3.01 g, $20.2 \mathrm{mmol})$ in $\mathrm{CH}_{2} \mathrm{Cl}_{2}(20 \mathrm{~mL})$ was mixed with $\mathrm{Et}_{3} \mathrm{~N}$ (5.61 mL, $\left.40.4 \mathrm{mmol}\right)$, dimethylaminopyridine $(0.247 \mathrm{~g}, 2.02 \mathrm{mmol})$, and trimethylacetyl chloride $(4.96 \mathrm{~mL}, 40.1 \mathrm{mmol})$ to give ester $\mathbf{S 2 3}$ as a colorless oil $(4.69 \mathrm{~g}, 99 \%)$ :

${ }^{1} \mathrm{H}$ NMR $\left(400 \mathrm{MHz}, \mathrm{CDCl}_{3}\right) \delta 7.23(\mathrm{~d}, J=7.8,2 \mathrm{H}), 7.15(\mathrm{~d}, J=7.8,2 \mathrm{H}), 6.19(\mathrm{~d}, J=5.5,1 \mathrm{H}), 5.98$ (ddd, $J=16.3,10.5,5.6,1 \mathrm{H}), 5.28(\mathrm{~d}, J=17.2,1 \mathrm{H}), 5.19(\mathrm{~d}, J=10.4,1 \mathrm{H}), 2.33(\mathrm{~s}, 3 \mathrm{H}), 1.22(\mathrm{~s}$, 9H);

${ }^{13} \mathrm{C}$ NMR (100 MHz, $\left.\mathrm{CDCl}_{3}\right) \delta 177.4(\mathrm{C}), 137.8(\mathrm{C}), 136.8(\mathrm{C}), 136.4(\mathrm{CH}), 129.3(\mathrm{CH}), 126.9$ $(\mathrm{CH}), 116.3\left(\mathrm{CH}_{2}\right), 75.8(\mathrm{CH}), 38.9(\mathrm{C}), 27.2\left(\mathrm{CH}_{3}\right), 21.3\left(\mathrm{CH}_{3}\right)$;

IR (ATR) 2975, 1729, 1103, 812, $771 \mathrm{~cm}^{-1}$;

HRMS (APCl) $m / z$ calcd for $\mathrm{C}_{10} \mathrm{H}_{11}\left((\mathrm{M}+\mathrm{H})-\mathrm{C}_{5} \mathrm{H}_{10} \mathrm{O}_{2}\right)^{+} 131.0855$, found 131.0852 . 
Anal. Calcd for $\mathrm{C}_{15} \mathrm{H}_{20} \mathrm{O}_{2}: \mathrm{C}, 77.55 ; \mathrm{H}, 8.68$. Found: $\mathrm{C}, 77.49 ; \mathrm{H}, 8.62$.<smiles>C=CC(O)c1cccc2ccccc12</smiles><smiles>CCO[N+](C)(C)CC(=O)Cl</smiles><smiles>C=CC(OC(=O)C(C)(C)C)c1c(Br)ccc2ccccc12</smiles>

1-(Naphthalen-1-yl)allyl pivaloate (S24). Following the general procedure for esterification, allylic alcohol S18 $(2.1 \mathrm{~g}, 15 \mathrm{mmol})$ in $\mathrm{CH}_{2} \mathrm{Cl}_{2}(15 \mathrm{~mL})$ was mixed with $\mathrm{Et}_{3} \mathrm{~N}(4.2 \mathrm{~mL}, 30 \mathrm{mmol})$, dimethylaminopyridine $(0.183 \mathrm{mg}, 1.50 \mathrm{mmol})$, and trimethylacetyl chloride $(2.55 \mathrm{~mL}, 30.0 \mathrm{mmol})$ to give ester S24 as a colorless oil $(2.71 \mathrm{~g}, 97 \%)$ :

${ }^{1} \mathrm{H} \mathrm{NMR}\left(400 \mathrm{MHz}, \mathrm{CDCl}_{3}\right) \delta 8.13(\mathrm{~d}, J=8.4,1 \mathrm{H}), 7.88(\mathrm{~d}, J=7.2,1 \mathrm{H}), 7.83(\mathrm{~d}, J=8.4,1 \mathrm{H}), 7.60$ (d, $J=7.1,1 \mathrm{H}), 7.46-7.55(\mathrm{~m}, 3 \mathrm{H}), 6.95(\mathrm{~d}, J=5.8,1 \mathrm{H}), 6.19(\mathrm{ddd}, J=15.8,10.6,5.2,1 \mathrm{H}), 5.30$ (ddt overlapped, $J=18.9,10.8,1.6,2 \mathrm{H}), 1.25(\mathrm{~s}, 9 \mathrm{H})$;

${ }^{13} \mathrm{C} \mathrm{NMR}\left(100 \mathrm{MHz}, \mathrm{CDCl}_{3}\right) \delta 177.7(\mathrm{C}), 136.5(\mathrm{C}), 135.2(\mathrm{C}), 134.3(\mathrm{C}), 131.1(\mathrm{CH}), 129.2(\mathrm{CH})$, $129.1(\mathrm{CH}), 126.5(\mathrm{CH}), 126.1(\mathrm{CH}), 125.6(\mathrm{CH}), 125.6(\mathrm{CH}), 124.3(\mathrm{CH}), 117.2\left(\mathrm{CH}_{2}\right), 73.8(\mathrm{CH})$, $39.3(\mathrm{C}), 27.5\left(\mathrm{CH}_{3}\right)$;

HRMS (APCl) m/z: [[M + H] $\left.-\mathrm{C}_{5} \mathrm{H}_{10} \mathrm{O}_{2}\right]^{+}$Calcd for $\mathrm{C}_{13} \mathrm{H}_{11}$ 167.0855; Found 167.0843.<smiles>C=CC(O)c1ccc(C)cc1</smiles><smiles>CCN(CC)CCO[N+](=O)[O-]</smiles><smiles>C=CC(OC(=O)c1ccc([N+](=O)[O-])cc1)c1ccc(C)cc1</smiles>

1-(p-Tolyl)allyl 4-nitrobenzoate (S25). Following the general procedure for esterification, allylic alcohol S16 $(0.50 \mathrm{~g}, 3.3 \mathrm{mmol})$ in $\mathrm{CH}_{2} \mathrm{Cl}_{2}(3.5 \mathrm{~mL})$ was mixed with $\mathrm{Et}_{3} \mathrm{~N}(0.940 \mathrm{~mL}, 6.75 \mathrm{mmol})$, dimethylaminopyridine $(0.04 \mathrm{~g}, 0.3 \mathrm{mmol})$, and p-nitrobenzoyl chloride $(1.25 \mathrm{~g}, 6.75 \mathrm{mmol})$ to provide ether $\mathbf{S} 25$ as a colorless oil $(1.02 \mathrm{~g}, 99 \%)$. The analytical data obtained are consistent with those reported: 6

${ }^{1} \mathrm{H}$ NMR $\left(400 \mathrm{MHz}, \mathrm{CDCl}_{3}\right) \delta 8.29(\mathrm{~d}, J=8.9,2 \mathrm{H}), 8.25(\mathrm{~d}, J=8.9,2 \mathrm{H}), 7.35(\mathrm{~d}, J=7.7,2 \mathrm{H}), 7.21$ $(\mathrm{d}, J=7.7,2 \mathrm{H}), 6.50(\mathrm{~d}, J=5.9,1 \mathrm{H}), 6.14$ (ddd, $J=16.9,10.7,6.2,1 \mathrm{H}), 5.36(\mathrm{~d}, J=19.2,1 \mathrm{H})$, $5.31(\mathrm{~d}, J=11.3,1 \mathrm{H}), 2.36(\mathrm{~s}, 3 \mathrm{H})$;

${ }^{13} \mathrm{C}$ NMR $\left(100 \mathrm{MHz}, \mathrm{CDCl}_{3}\right) \delta 163.8(\mathrm{C}), 150.7(\mathrm{C}), 138.6(\mathrm{C}), 135.9\left(\mathrm{CH}_{2}\right), 135.4(\mathrm{C}), 131.4(\mathrm{C})$, $130.9(\mathrm{CH}), 129.6(\mathrm{CH}), 127.4(\mathrm{CH}), 123.7(\mathrm{CH}), 116.7(\mathrm{CH}), 77.9(\mathrm{CH}), 22.8\left(\mathrm{CH}_{3}\right)$;

IR (ATR) 2976, 1725, 1526, 1344, $1268 \mathrm{~cm}^{-1}$.<smiles>C=CC(O)c1ccc(C)cc1</smiles><smiles>CCN(CC)CCOC</smiles><smiles>C=CC(OC(=O)c1ccc(OC)cc1)c1ccc(C)cc1</smiles> 
1-(p-Tolyl)allyl 4-methoxybenzoate (S26). Following the general procedure for esterification, allylic alcohol $\mathbf{S} 16(3.01 \mathrm{~g}, 20.2 \mathrm{mmol})$ in $\mathrm{CH}_{2} \mathrm{Cl}_{2}(20 \mathrm{~mL})$ was mixed with $\mathrm{Et}_{3} \mathrm{~N}(5.62 \mathrm{~mL}, 40.4$ $\mathrm{mmol})$, dimethylaminopyridine $(0.247 \mathrm{~g}, 2.02 \mathrm{mmol})$, and $p$-methoxybenzoyl chloride $(5.48 \mathrm{~mL}$, $40.4 \mathrm{mmol})$ to provide ether $\mathbf{S} 26$ as a colorless oil $(4.73 \mathrm{~g}, 83 \%)$ :

${ }^{1} \mathrm{H}$ NMR $\left(400 \mathrm{MHz}, \mathrm{CDCl}_{3}\right) \delta 8.06(\mathrm{~d}, J=8.8,2 \mathrm{H}), 7.35(\mathrm{~d}, J=7.8,2 \mathrm{H}), 7.19(\mathrm{~d}, J=7.8,2 \mathrm{H}), 6.93$ $(\mathrm{d}, J=8.8,2 \mathrm{H}), 6.47(\mathrm{~d}, J=6.8,1 \mathrm{H}), 6.12$ (ddd, $J=16.4,10.5,5.8,1 \mathrm{H}), 5.38(\mathrm{~d}, J=16.9,1 \mathrm{H})$, $5.28(\mathrm{dt}, J=10.3,1.4,1 \mathrm{H}), 3.87(\mathrm{~s}, 3 \mathrm{H}), 2.36(\mathrm{~s}, 3 \mathrm{H})$;

${ }^{13} \mathrm{C}$ NMR (100 MHz, CDCl $)$ $) 165.4(\mathrm{C}), 163.6(\mathrm{C}), 138.1(\mathrm{C}), 136.8(\mathrm{C}), 136.4(\mathrm{C}), 131.9(\mathrm{CH})$, $129.4(\mathrm{CH}), 127.3(\mathrm{CH}), 122.9(\mathrm{CH}), 116.7(\mathrm{CH}), 113.7\left(\mathrm{CH}_{2}\right), 76.4(\mathrm{CH}), 55.6\left(\mathrm{CH}_{3}\right), 21.3\left(\mathrm{CH}_{3}\right)$; IR (ATR) 1709, 1605, 1249, 1095, $767 \mathrm{~cm}^{-1}$;

HRMS (APCI) m/z: [[M + H] $\left.-\mathrm{C}_{8} \mathrm{H}_{8} \mathrm{O}_{3}\right]^{+}$Calcd for $\mathrm{C}_{10} \mathrm{H}_{11}$ 131.0855; Found 131.0852 .

Anal. Calcd for $\mathrm{C}_{18} \mathrm{H}_{18} \mathrm{O}_{3}$ : C, 76.57; $\mathrm{H}, 6.43$. Found: $\mathrm{C}, 76.32 ; \mathrm{H}, 6.29$.<smiles>C=CC(O)c1ccc(C)cc1</smiles>

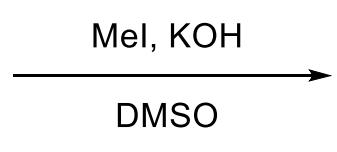<smiles>C=CC(OC)c1ccc(C)cc1</smiles>

1-(1-Methoxyallyl)-4-methylbenzene (S27). This procedure was adapted by that reported by Smith et. al. ${ }^{7}$ To a solution of alcohol $\mathbf{S 1 6}(0.50 \mathrm{~g}, 3.4 \mathrm{mmol})$ in DMSO $(6.75 \mathrm{~mL})$ was added crushed $\mathrm{KOH}$ pellets $(0.796 \mathrm{~g}, 13.5 \mathrm{mmol})$. After $15 \mathrm{~min}$, Mel $(0.42 \mathrm{~mL}, 6.8 \mathrm{mmol})$ was added dropwise over $20 \mathrm{~min}$. After $12 \mathrm{~h}$, the reaction mixture was diluted with $\mathrm{H}_{2} \mathrm{O}(20 \mathrm{~mL})$ and the aqueous layer was extracted with EtOAc $(2 \times 30 \mathrm{~mL})$. The combined organic layers were washed with brine, dried over $\mathrm{Na}_{2} \mathrm{SO}_{4}$, filtered through cotton, and concentrated in vacuo to provide a yellow oil. The oil was purified using column chromatography (5:95 EtOAc:hexanes) to provide ether S27 as a yellow oil $(0.53 \mathrm{~g}, 97 \%)$. Spectral data obtained are consistent with those reported: ${ }^{8}$ ${ }^{1} \mathrm{H}$ NMR $\left(400 \mathrm{MHz}, \mathrm{CDCl}_{3}\right) \delta 7.23(\mathrm{~d}, J=8.5,2 \mathrm{H}), 7.17(\mathrm{~d}, J=8.5,2 \mathrm{H}), 5.94$ (ddd, $J=17.1,10.3$, $6.7,1 \mathrm{H}), 5.28(\mathrm{dt}, J=17.2,1.5,1 \mathrm{H}), 5.20(\mathrm{dt}, J=9.6,1.5,1 \mathrm{H}), 4.60(\mathrm{~d}, J=6.4,1 \mathrm{H}), 3.33(\mathrm{~s}, 3 \mathrm{H})$, $2.36(\mathrm{~s}, 3 \mathrm{H})$;

${ }^{13} \mathrm{C}$ NMR (100 MHz, CDCl $\left.{ }_{3}\right) \delta 139.1(\mathrm{C}), 137.9(\mathrm{C}), 137.5(\mathrm{CH}), 129.3(\mathrm{CH}), 126.9(\mathrm{CH}), 116.2$ $\left(\mathrm{CH}_{2}\right), 84.7(\mathrm{CH}), 56.4\left(\mathrm{CH}_{3}\right), 21.3\left(\mathrm{CH}_{3}\right)$;

IR (ATR) 2959, 1731, 1152, 1068, $813 \mathrm{~cm}^{-1}$;

HRMS (APCl) m/z: [[M + H] $\left.-\mathrm{CH}_{4} \mathrm{O}\right]^{+}$Calcd for $\mathrm{C}_{10} \mathrm{H}_{11}$ 131.0855; Found 131.0857.

General procedure for ozonolysis of alkenes to aldehydes. This procedure was adapted from that reported by Saha et. al. ${ }^{8} \mathrm{~A}$ solution of the allylic ester (1 equiv) in $\mathrm{CH}_{2} \mathrm{Cl}_{2}(0.2 \mathrm{M})$ was cooled to $-78^{\circ} \mathrm{C}$ and then a steady stream of ozone was applied for 30 min until saturation and a purple color appeared. The reaction mixture was warmed to room temperature over $30 \mathrm{~min}$, purged with nitrogen, and then $\mathrm{Me}_{2} \mathrm{~S}$ was added (10 equiv). After $5 \mathrm{~h}$, the reaction mixture was concentrated in vacuo to provide the aldehyde as a clear yellow oil which was used without further purification because they decomposed on silica gel, deactivated silica gel, and basified silica gel. Because these aldehydes were not stable, they were not characterized and were subjected to the next reaction immediately.

General procedure for acetal protection of aldehydes. This procedure was adapted from that reported by Wu et. al. ${ }^{9}$ To a solution of the unpurified aldehyde (1 equiv) in triethyl orthoformate (10 equiv, trimethyl orthoformate could also be used) was added $p$-toluenesulfonic acid ( 0.5 equiv) and $\mathrm{EtOH}\left(0.1 \mathrm{M}\right.$, MeOH could also be used). After $8 \mathrm{~h}$, a saturated solution of $\mathrm{NaHCO}_{3}(20 \mathrm{~mL}$ per $20 \mathrm{mmol})$ was added and the aqueous layer was extracted with $\mathrm{CH}_{2} \mathrm{Cl}_{2}(2 \times 30 \mathrm{~mL}$ per 20 $\mathrm{mmol})$. The organic layers were combined, washed with brine $(20 \mathrm{~mL}$ per $20 \mathrm{mmol})$, dried over 
$\mathrm{Na}_{2} \mathrm{SO}_{4}$, filtered through cotton, and concentrated in vacuo. The residue was purified by flash column chromatography (3:97 EtOAc:hexanes) to provide the acetal as a colorless oil.

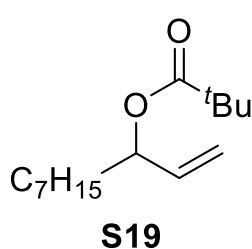

S19

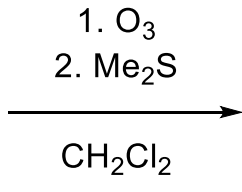

S28



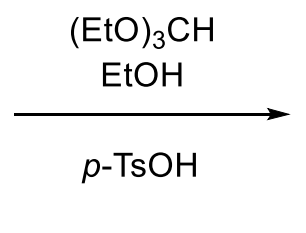<smiles>CCCCC(OC(=O)Br)C(OCC)OCC</smiles>

4b

1,1-Diethoxynonan-2-yl pivaloate (4b). Following the general procedure for ozonolysis, ester $\mathbf{S} 19(5.97 \mathrm{~g}, 25.0 \mathrm{mmol})$ was treated with $\mathrm{O}_{3}$ to provide aldehyde $\mathbf{S 2 8}$ as a yellow oil. Following the general procedure for acetal formation, aldehyde $\mathbf{S 2 8}(5.97 \mathrm{~g}, 25.0 \mathrm{mmol})$ was mixed with triethyl orthoformate $(41 \mathrm{~mL}, 0.25 \mathrm{~mol})$, $p$-toluenesulfonic acid $(2.35 \mathrm{~g}, 12.5 \mathrm{mmol})$, and $\mathrm{EtOH}(2.5$ $\mathrm{mL})$ to produce acetal $\mathbf{4 b}$ as a colorless oil $(4.28 \mathrm{~g}, 54 \%)$ :

${ }^{1} \mathrm{H}$ NMR $\left(400 \mathrm{MHz}, \mathrm{CDCl}_{3}\right) \delta$ 4.91-4.96 (m, 1H), $4.31(\mathrm{~d}, J=5.7,1 \mathrm{H}), 3.62-3.74(\mathrm{~m}, 2 \mathrm{H}), 3.51-$ $3.60(\mathrm{~m}, 2 \mathrm{H}), 1.27-1.26(\mathrm{~m}, 12 \mathrm{H}), 1.22(\mathrm{~s}, 9 \mathrm{H}), 1.13-1.21(\mathrm{~m}, 6 \mathrm{H}), 0.87(\mathrm{t}, J=7.2,3 \mathrm{H})$;

${ }^{13} \mathrm{C} \mathrm{NMR}\left(100 \mathrm{MHz}, \mathrm{CDCl}_{3}\right)$ ठ $178.1(\mathrm{C}), 102.5(\mathrm{CH}), 72.8(\mathrm{CH}), 63.4\left(\mathrm{CH}_{2}\right), 62.2\left(\mathrm{CH}_{2}\right), 38.9(\mathrm{C})$, $31.9\left(\mathrm{CH}_{2}\right), 29.5\left(\mathrm{CH}_{2}\right), 29.3\left(\mathrm{CH}_{2}\right), 29.1\left(\mathrm{CH}_{2}\right), 27.4\left(\mathrm{CH}_{2}\right), 27.3\left(\mathrm{CH}_{2}\right), 25.2\left(\mathrm{CH}_{3}\right), 22.7\left(\mathrm{CH}_{3}\right)$, $15.4\left(\mathrm{CH}_{3}\right), 14.2\left(\mathrm{CH}_{3}\right)$;

IR (ATR) 2927, 1729, 1118, 1065, $1033 \mathrm{~cm}^{-1}$;

HRMS (APCl) m/z: [[M + H] $\left.-\mathrm{C}_{2} \mathrm{H}_{6} \mathrm{O}\right]^{+}$Calcd for $\mathrm{C}_{16} \mathrm{H}_{31} \mathrm{O}_{3}$ 271.2268; Found 271.2287.<smiles>C=CC(OC(=O)CBr)C1CCCCC1</smiles>

S20

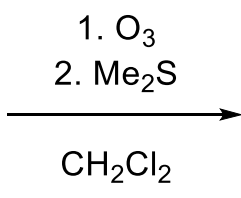

$\mathrm{CH}_{2} \mathrm{Cl}_{2}$<smiles>CC(C)(C)C(=O)OC(C=O)C1CCCCC1</smiles>

S29

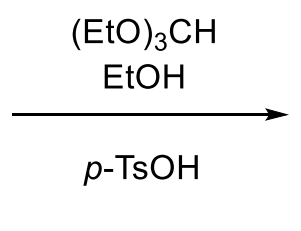

1-Cyclohexyl-2,2-diethoxyethyl pivaloate (4e). Following the general procedure for ozonolysis, ester $\mathbf{S} 20$ (2.50 g, $11.1 \mathrm{mmol})$ was treated with $\mathrm{O}_{3}$ to provide aldehyde $\mathbf{S 2 9}$ as a yellow oil. Following the general procedure for acetal formation, aldehyde $\mathbf{S 2 9}(2.50 \mathrm{~g}, 11.1 \mathrm{mmol})$ was mixed with triethyl orthoformate $(18 \mathrm{~mL}, 0.11 \mathrm{~mol})$, $p$-toluenesulfonic acid $(1.05 \mathrm{~g}, 5.50 \mathrm{mmol})$, and $\mathrm{EtOH}(1.0 \mathrm{~mL})$ to provide acetal $4 \mathrm{e}$ as a colorless oil $(0.65 \mathrm{~g}, 20 \%)$ :

${ }^{1} \mathrm{H}$ NMR $\left(400 \mathrm{MHz}, \mathrm{CDCl}_{3}\right) \delta 4.89(\mathrm{dd}, J=7.1,3.6,1 \mathrm{H}), 4.55(\mathrm{~d}, J=7.1,1 \mathrm{H}), 3.59-3.73(\mathrm{~m}, 2 \mathrm{H})$, 3.47-3.59 (m, 2H), 1.64-1.77 (m, 7H), $1.09(\mathrm{~m}, 1 \mathrm{H}), 1.23(\mathrm{~s}, 9 \mathrm{H}), 1.21(\mathrm{t}, J=7.1,3 \mathrm{H}), 1.15(\mathrm{t}, J$ $=7.1,3 \mathrm{H}), 0.86-1.03(\mathrm{~m}, 3 \mathrm{H})$;

${ }^{13} \mathrm{C} \mathrm{NMR}\left(100 \mathrm{MHz}, \mathrm{CDCl}_{3}\right) \delta 177.8(\mathrm{C}), 100.7(\mathrm{CH}), 74.8(\mathrm{CH}), 62.6\left(\mathrm{CH}_{2}\right), 61.3\left(\mathrm{CH}_{2}\right), 39.1(\mathrm{C})$, 38.3 (CH), 30.1 $\left(\mathrm{CH}_{2}\right)$, $27.4\left(\mathrm{CH}_{3}\right), 26.9\left(\mathrm{CH}_{2}\right), 26.6\left(\mathrm{CH}_{2}\right), 26.4\left(\mathrm{CH}_{2}\right), 26.3\left(\mathrm{CH}_{2}\right), 15.4\left(\mathrm{CH}_{3}, \mathrm{CH}_{3}\right.$ as determined by DEPT and HSQC);

IR (ATR) 2927, 1730, 1116, 1122, $1056 \mathrm{~cm}^{-1}$;

HRMS (APCl) m/z: [[M + H] $\left.-\mathrm{C}_{2} \mathrm{H}_{6} \mathrm{O}\right]^{+}$Calcd for $\mathrm{C}_{15} \mathrm{H}_{27} \mathrm{O}_{3}$ 255.1955; Found 255.1954.<smiles>C=CC(Cc1ccccc1)OC(=O)Br</smiles>

$(R)-\mathbf{S} 21$

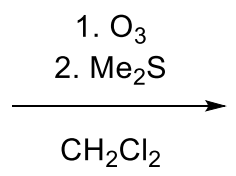

-3-phe<smiles>CC(=O)C(Cc1ccccc1)OC(=O)Br</smiles>

$(R)-\mathrm{S} 30^{\mathrm{H}}$

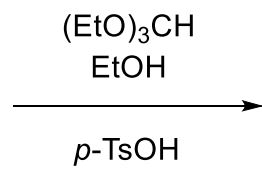

$p-\mathrm{TsOH}$

$(R)$-1,1-Diethoxy-3-phenylpropan-2-yl pivaloate $((R)-4 f)$. Following the general procedure for ozonolysis, ester $(R)$-S21 (0.95 g, $4.4 \mathrm{mmol}$ ) was treated with $\mathrm{O}_{3}$ to provide aldehyde $(\mathrm{R})$-S30 as 
a yellow oil. Following the general procedure for acetal formation, aldehyde $(R)$-S30 $(0.95 \mathrm{~g}, 4.4$ $\mathrm{mmol}$ ) was mixed with triethyl orthoformate $(7.0 \mathrm{~mL}, 0.044 \mathrm{~mol}), p$-toluenesulfonic acid $(0.42 \mathrm{~g}$, $2.2 \mathrm{mmol})$, and EtOH $(1 \mathrm{~mL})$ to provide acetal $(R)-4 \mathrm{f}$ as a colorless oil $(0.864 \mathrm{~g}, 65 \%)$ :

${ }^{1} \mathrm{H}$ NMR $\left(400 \mathrm{MHz}, \mathrm{CDCl}_{3}\right) \delta 7.26-7.17(\mathrm{~m}, 5 \mathrm{H}), 5.14-5.19(\mathrm{~m}, 1 \mathrm{H}), 4.47(\mathrm{~d}, J=5.5,1 \mathrm{H}), 3.67-$ $3.78(\mathrm{~m}, 2 \mathrm{H}), 3.53-3.61(\mathrm{~m}, 2 \mathrm{H}), 3.10(\mathrm{dd}, J=14.2,3.4,1 \mathrm{H}), 2.88(\mathrm{dd}, J=14.2,9.2,1 \mathrm{H}), 1.25$ (t, $J=7.1,3 \mathrm{H}), 1.19(\mathrm{t}, J=7.1,3 \mathrm{H}), 1.08(\mathrm{~s}, 9 \mathrm{H})$;

${ }^{13} \mathrm{C}$ NMR (100 MHz, $\left.\mathrm{CDCl}_{3}\right) \delta 177.7(\mathrm{C}), 137.7(\mathrm{C}), 129.7(\mathrm{CH}), 128.3(\mathrm{CH}), 126.4(\mathrm{CH}), 102.0$ $(\mathrm{CH}), 73.2(\mathrm{CH}), 63.7\left(\mathrm{CH}_{2}\right), 62.6\left(\mathrm{CH}_{2}\right), 38.8(\mathrm{C}), 35.4\left(\mathrm{CH}_{2}, \mathrm{CH}_{2}\right.$ as determined by DEPT and HSQC), $27.2\left(\mathrm{CH}_{3}\right), 15.5\left(\mathrm{CH}_{3}, \mathrm{CH}_{3}\right.$ as determined by DEPT and HSQC);

IR (ATR) 2975, 1728, 1157, 1063, $1031 \mathrm{~cm}^{-1}$;

HRMS (APCl) m/z: [[M + H] $\left.-\mathrm{C}_{2} \mathrm{H}_{6} \mathrm{O}\right]^{+}$Calcd for $\mathrm{C}_{16} \mathrm{H}_{23} \mathrm{O}_{3}$ 263.1642; Found 263.1649.

Anal. Calcd for $\mathrm{C}_{18} \mathrm{H}_{28} \mathrm{O}_{4}$ : C, 70.10; $\mathrm{H}, 9.15$. Found: $\mathrm{C}, 69.91 ; \mathrm{H}, 9.32$.

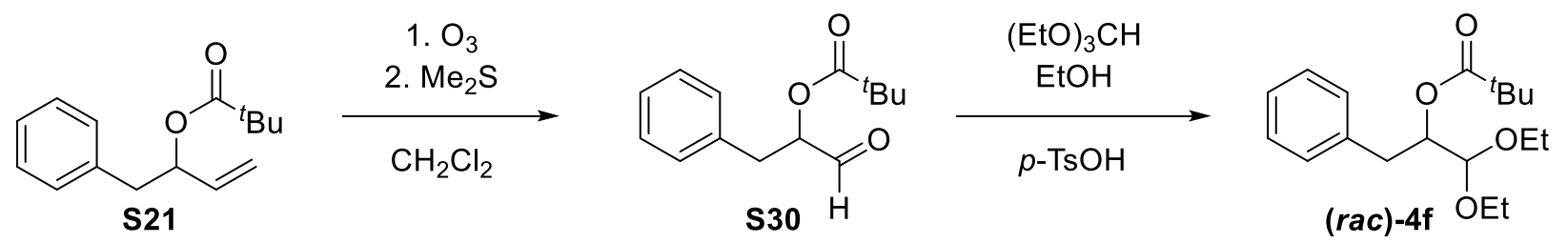

1,1-Diethoxy-3-phenylpropan-2-yl pivaloate ((rac)-4f). Following the general procedure for ozonolysis, ester ( $\mathrm{rac}$ )-S21 $\left(0.844 \mathrm{~g}, 3.89 \mathrm{mmol}\right.$ ) was treated with $\mathrm{O}_{3}$ to provide aldehyde $(\mathrm{rac})$ S30 as a yellow oil. Following the general procedure for acetal formation, aldehyde ( $\mathrm{rac}$ )-S30 $(0.844 \mathrm{~g}, 3.89 \mathrm{mmol})$ was mixed with triethyl orthoformate $(6.5 \mathrm{~mL}, 0.039 \mathrm{~mol}), p$-toluenesulfonic acid $(0.4 \mathrm{~g}, 0.002 \mathrm{mmol})$, and $\mathrm{EtOH}(1 \mathrm{~mL})$ to provide acetal $(\mathrm{rac})-\mathbf{4 f}$ as a colorless oil $(0.586 \mathrm{~g}$, $49 \%)$. Spectral data obtained are consistent with that reported above for the enantiomerically enriched compound.<smiles>C=CC(Br)(Br)OC(=O)CC(C)(C)C</smiles>

S22

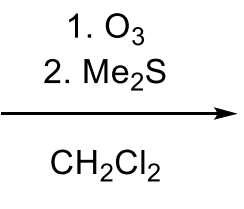

rac<smiles>O=CC(Br)(Br)C(=O)OC(Br)CBr</smiles>

S31

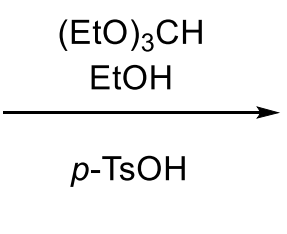
1,1-Diethoxy-3,3-dimethylbutan-2-yl pivaloate (4d). Following the general procedure for
ozonolysis, ester $\mathbf{S} 22(0.954 \mathrm{~g}, 4.10 \mathrm{mmol})$ was treated with $\mathrm{O}_{3}$ to provide aldehyde $\mathrm{S} 31$ as a yellow oil. Following the general procedure for acetal formation, aldehyde S31 was mixed with triethyl orthoformate $(6.8 \mathrm{~mL}, 41 \mathrm{mmol}), p$-toluenesulfonic acid $(0.387 \mathrm{~g}, 2.20 \mathrm{mmol})$, and EtOH $(0.5 \mathrm{~mL})$ to provide acetal $4 \mathrm{~d}$ as a colorless oil $(0.96 \mathrm{~g}, 86 \%)$ :

${ }^{1} \mathrm{H}$ NMR $\left(400 \mathrm{MHz}, \mathrm{CDCl}_{3}\right) \delta 4.78(\mathrm{~d}, J=5.9,1 \mathrm{H}), 4.52(\mathrm{~d}, J=5.9,1 \mathrm{H}), 3.66-3.71(\mathrm{~m}, 1 \mathrm{H}), 3.55-$ $3.62(\mathrm{~m}, 1 \mathrm{H}), 3.48-3.54(\mathrm{~m}, 2 \mathrm{H}), 1.24(\mathrm{~s}, 9 \mathrm{H}), 1.13-1.21(\mathrm{~m}, 6 \mathrm{H}), 0.98(\mathrm{~s}, 9 \mathrm{H})$;

${ }^{13} \mathrm{C}$ NMR $\left(100 \mathrm{MHz}, \mathrm{CDCl}_{3}\right) \delta 177.5(\mathrm{C}), 101.3(\mathrm{CH}), 76.8(\mathrm{CH}), 62.4\left(\mathrm{CH}_{2}\right), 61.1\left(\mathrm{CH}_{2}\right), 39.1(\mathrm{C})$, $34.0(\mathrm{C}), 27.5\left(\mathrm{CH}_{3}\right), 26.7\left(\mathrm{CH}_{3}\right), 15.4\left(\mathrm{CH}_{3}\right), 15.3\left(\mathrm{CH}_{3}\right)$;

IR (ATR) 2973, 1731, 1162, 1139, $999 \mathrm{~cm}^{-1}$;

HRMS (APCl) m/z: [[M + H] $\left.-\mathrm{C}_{2} \mathrm{H}_{6} \mathrm{O}\right]^{+}$Calcd for $\mathrm{C}_{13} \mathrm{H}_{25} \mathrm{O}_{3}$ 229.1798; Found 229.1805. 


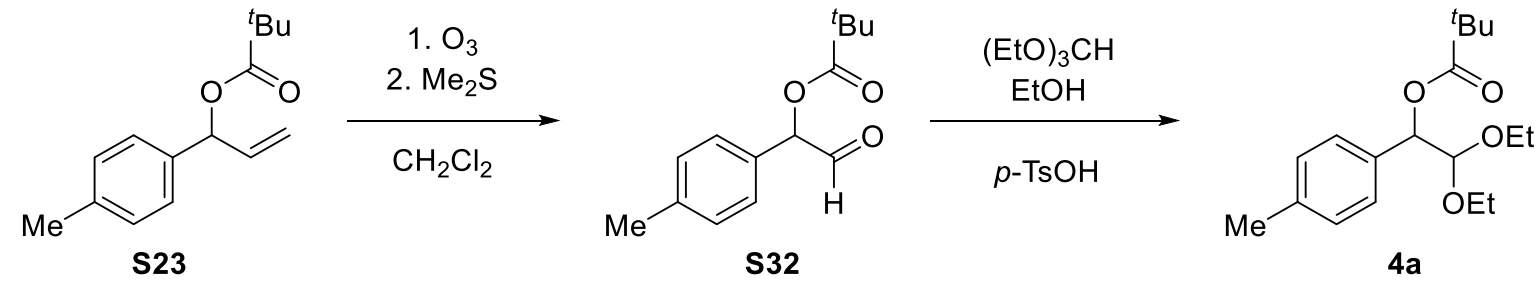

2,2-Diethoxy-1-(p-tolyl)ethyl pivaloate (4a). Following the general procedure for ozonolysis, ester $\mathbf{S} 23(4.96 \mathrm{~g}, 20.0 \mathrm{mmol})$ was treated with $\mathrm{O}_{3}$ to provide aldehyde $\mathbf{S} 32$ as a yellow oil. Following the general procedure for acetal formation, aldehyde $\mathbf{S} 32(4.96 \mathrm{~g}, 20.0 \mathrm{mmol})$ was mixed with triethyl orthoformate $(33 \mathrm{~mL}, 0.20 \mathrm{~mol})$, $p$-toluenesulfonic acid $(1.91 \mathrm{~g}, 10.0 \mathrm{mmol})$, and $\mathrm{EtOH}(2 \mathrm{~mL})$ to provide acetal $4 \mathrm{a}$ as a colorless oil $(3.92 \mathrm{~g}, 64 \%)$ :

${ }^{1} \mathrm{H}$ NMR $\left(400 \mathrm{MHz}, \mathrm{CDCl}_{3}\right) \delta 7.27(\mathrm{~d}, J=7.6,2 \mathrm{H}), 7.13(\mathrm{~d}, J=7.6,2 \mathrm{H}), 5.72(\mathrm{~d}, J=6.6,1 \mathrm{H}), 4.63$ $(\mathrm{d}, J=6.6,1 \mathrm{H}), 3.65-3.72(\mathrm{~m}, 1 \mathrm{H}), 3.58-3.65(\mathrm{~m}, 2 \mathrm{H}), 3.35(\mathrm{dq}, J=9.4,7.1,1 \mathrm{H}), 2.34(\mathrm{~s}, 3 \mathrm{H})$, $1.23(\mathrm{~s}, 9 \mathrm{H}), 1.21(\mathrm{t}, J=7.1,3 \mathrm{H}), 1.04(\mathrm{t}, J=7.1,3 \mathrm{H})$;

${ }^{13} \mathrm{C}$ NMR (100 MHz, $\left.\mathrm{CDCl}_{3}\right) \delta 177.1(\mathrm{C}), 137.7(\mathrm{C}), 134.3(\mathrm{C}), 128.9(\mathrm{CH}), 127.6(\mathrm{CH}), 103.3$ $(\mathrm{CH}), 74.6(\mathrm{CH}), 63.9\left(\mathrm{CH}_{2}\right), 62.3\left(\mathrm{CH}_{2}\right), 38.9(\mathrm{C}), 27.2\left(\mathrm{CH}_{3}\right), 21.3\left(\mathrm{CH}_{3}\right), 15.4\left(\mathrm{CH}_{3}\right), 15.2\left(\mathrm{CH}_{3}\right)$. IR (ATR) 2974, 1732, 1116, 1064, $811 \mathrm{~cm}^{-1}$;

HRMS (APCl) m/z: [[M + H] $\left.-\mathrm{C}_{2} \mathrm{H}_{6} \mathrm{O}\right]^{+}$Calcd for $\mathrm{C}_{16} \mathrm{H}_{23} \mathrm{O}_{3}$ 263.1642; Found 263.1635.

Anal. Calcd for $\mathrm{C}_{18} \mathrm{H}_{28} \mathrm{O}_{4}$ : C, 70.10; $\mathrm{H}, 9.15$. Found: $\mathrm{C}, 70.10 ; \mathrm{H}, 9.18$.<smiles>C=CC(OC(=O)CC(C)(C)C)c1cccc2ccccc12</smiles>

S24



$\mathrm{CH}_{2} \mathrm{Cl}_{2}$<smiles>CC(C)(C)C(=O)OC(C=O)c1cccc2ccccc12</smiles>

S33

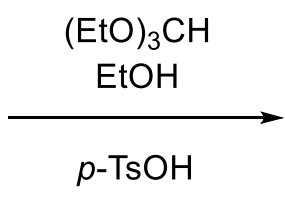

$4 c$

2,2-Diethoxy-1-(naphthalen-1-yl)ethyl pivaloate (4c). Following the general procedure for ozonolysis, ester $\mathbf{S} 24(2.4 \mathrm{~g}, 9.0 \mathrm{mmol})$ was treated with $\mathrm{O}_{3}$ to provide aldehyde $\mathbf{S} 33$ as a yellow oil. Following the general procedure for acetal formation, aldehyde S33 $(2.4 \mathrm{~g}, 9.0 \mathrm{mmol})$ was mixed with triethyl orthoformate $(15 \mathrm{~mL}, 90 \mathrm{mmol}), p$-toluenesulfonic acid $(0.856 \mathrm{~g}, 4.50 \mathrm{mmol})$, and $\mathrm{EtOH}(2 \mathrm{~mL})$ to provide acetal $4 \mathrm{c}$ as a colorless oil $(0.877 \mathrm{~g}, 28 \%)$ :

${ }^{1} \mathrm{H}$ NMR $\left(400 \mathrm{MHz}, \mathrm{CDCl}_{3}\right) \delta 8.31(\mathrm{~d}, J=8.5,1 \mathrm{H}), 7.84(\mathrm{~d}, J=7.7,1 \mathrm{H}), 7.81(\mathrm{~d}, J=7.2,1 \mathrm{H}), 7.60$ $(\mathrm{d}, J=6.9,1 \mathrm{H}), 7.44-7.54(\mathrm{~m}, 3 \mathrm{H}), 6.52(\mathrm{~d}, J=6.8,1 \mathrm{H}), 4.89(\mathrm{~d}, J=6.8,1 \mathrm{H}), 3.73-3.80(\mathrm{~m}, 1 \mathrm{H})$, 3.62-3.69 (m, 1H), 3.48-3.56 (m, 1H), 3.10-3.18 (m, 1H), $1.24(\mathrm{~s}, 9 \mathrm{H}), 1.23(\mathrm{t}, J=7.2,3 \mathrm{H}), 0.80$ (t, $J=7.2,3 \mathrm{H})$;

${ }^{13} \mathrm{C}$ NMR (150 MHz, $\left.\mathrm{CDCl}_{3}\right)$ ठ 177.2 (C), $133.82(\mathrm{C}), 133.79(\mathrm{C}), 131.7(\mathrm{C}), 128.8(\mathrm{CH}), 128.6$ $(\mathrm{CH}), 126.0(\mathrm{CH}), 125.8(\mathrm{CH}), 125.6(\mathrm{CH}), 125.1(\mathrm{CH}), 124.6(\mathrm{CH}), 103.8(\mathrm{CH}), 72.3(\mathrm{CH}), 64.3$ $\left(\mathrm{CH}_{2}\right), 62.5\left(\mathrm{CH}_{2}\right), 38.9(\mathrm{C}), 27.3\left(\mathrm{CH}_{3}\right), 15.4\left(\mathrm{CH}_{3}\right), 14.9\left(\mathrm{CH}_{3}\right)$;

IR (ATR) 2975, 1732, 1154, 1064, $776 \mathrm{~cm}^{-1}$;

HRMS (APCl) m/z: [[M + H] $\left.-\mathrm{C}_{2} \mathrm{H}_{6} \mathrm{O}\right]^{+}$Calcd for $\mathrm{C}_{19} \mathrm{H}_{23} \mathrm{O}_{3}$ 299.1624; Found 299.1620. 

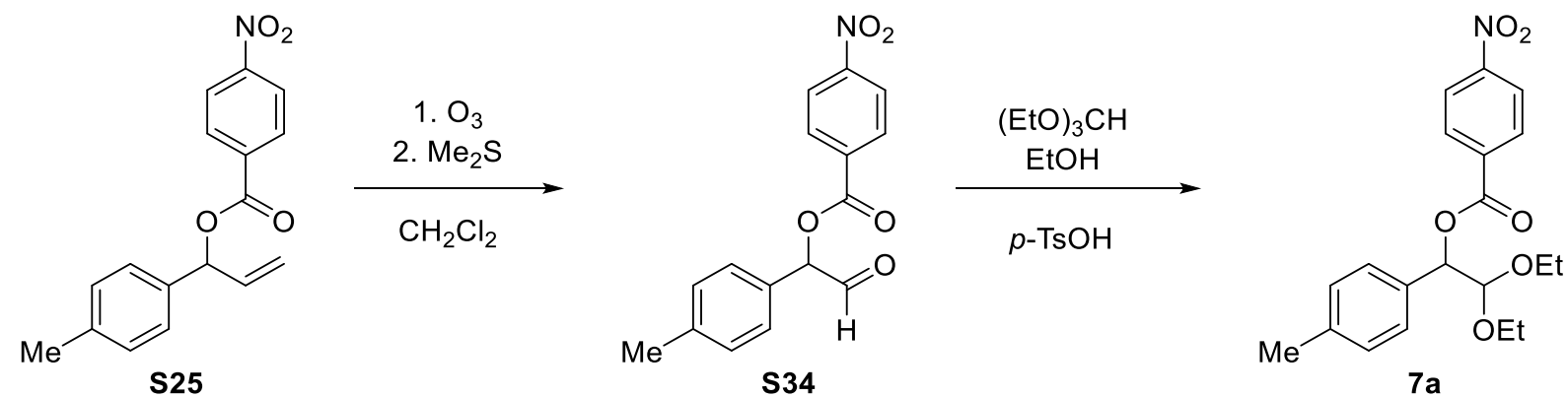

2,2-Diethoxy-1-(p-tolyl)ethyl 4-nitrobenzoate (7a). Following the general procedure for ozonolysis, ester $\mathbf{S} 25(1.2 \mathrm{~g}, 4.0 \mathrm{mmol})$ was treated with $\mathrm{O}_{3}$ to provide aldehyde $\mathbf{S} 34$ as a yellow oil. Following the general procedure for acetal formation, aldehyde S34 (1.2 g, $4.0 \mathrm{mmol})$ was mixed with triethyl orthoformate $(6.5 \mathrm{~mL}, 40 \mathrm{mmol})$, $p$-toluenesulfonic acid $(0.931 \mathrm{~g}, 5.01 \mathrm{mmol})$, and $\mathrm{EtOH}(0.5 \mathrm{~mL})$ to provide acetal $7 \mathrm{a}$ as a white solid $(0.356 \mathrm{~g}, 24 \%)$ :

$\mathrm{mp}=39-41^{\circ} \mathrm{C}$;

${ }^{1} \mathrm{H}$ NMR $\left(400 \mathrm{MHz}, \mathrm{CDCl}_{3}\right) \delta 8.30(\mathrm{~d}, J=8.7,2 \mathrm{H}), 8.25(\mathrm{~d}, J=8.7,2 \mathrm{H}), 7.38(\mathrm{~d}, J=7.8,2 \mathrm{H}), 7.18$ $(\mathrm{d}, J=7.8,2 \mathrm{H}), 6.01(\mathrm{~d}, J=6.1,1 \mathrm{H}), 4.80(\mathrm{~d}, J=6.3,1 \mathrm{H}), 3.69-3.80(\mathrm{~m}, 1 \mathrm{H}), 3.59-3.71(\mathrm{~m}, 2 \mathrm{H})$, 3.38-3.46 (m, 1H), $2.35(\mathrm{~s}, 3 \mathrm{H}), 1.19(\mathrm{t}, J=7.1,3 \mathrm{H}), 1.09(\mathrm{t}, J=7.1,3 \mathrm{H})$;

${ }^{13} \mathrm{C}$ NMR (100 MHz, CDCl $)$ $) 163.7(\mathrm{C}), 150.7(\mathrm{C}), 138.5(\mathrm{C}), 135.9(\mathrm{C}), 133.4(\mathrm{C}), 130.9(\mathrm{CH})$, $129.2(\mathrm{CH}), 127.8(\mathrm{CH}), 123.7(\mathrm{CH}), 103.2(\mathrm{CH}), 76.5(\mathrm{CH}), 64.1\left(\mathrm{CH}_{2}\right), 63.0\left(\mathrm{CH}_{2}\right), 21.4\left(\mathrm{CH}_{3}\right)$, $15.4\left(\mathrm{CH}_{3}\right), 15.2\left(\mathrm{CH}_{3}\right)$;

IR (ATR) 2974, 1727,1528, 1114, $1014 \mathrm{~cm}^{-1}$;

HRMS (APCl) m/z: [[M + H] $\left.-\mathrm{C}_{2} \mathrm{H}_{6} \mathrm{O}\right]^{+}$Calcd for $\mathrm{C}_{18} \mathrm{H}_{18} \mathrm{NO}_{5} 328.1179$; Found 328.1156.

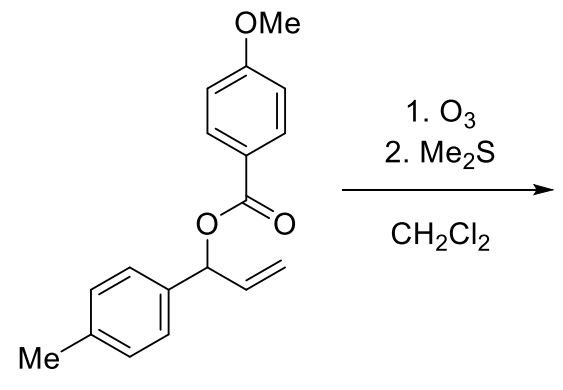

S26

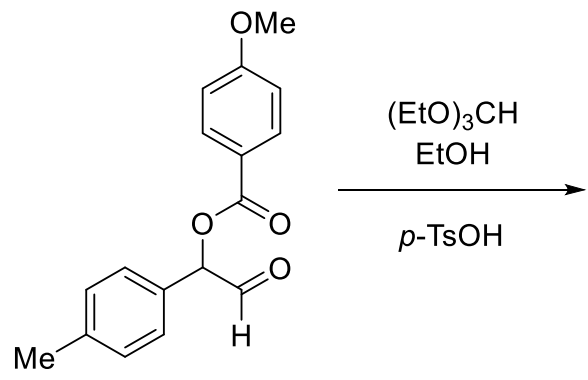

S35<smiles>CCOC(OCC)C(OC(=O)c1ccc(OC)cc1)c1ccc(C)cc1</smiles>

7b

2,2-Diethoxy-1-(p-tolyl)ethyl 4-methoxybenzoate (7b). Following the general procedure for ozonolysis, ester S26 $(5.68 \mathrm{~g}, 20.0 \mathrm{mmol})$ was treated with $\mathrm{O}_{3}$ to provide aldehyde $\mathbf{S} 35$ as a yellow oil. Following the general procedure for acetal formation, aldehyde S35 (5.68 g, $20.0 \mathrm{mmol})$ was mixed with triethyl orthoformate $(33 \mathrm{~mL}, 0.20 \mathrm{~mol}), p$-toluenesulfonic acid $(1.91 \mathrm{~g}, 10.0$ $\mathrm{mmol})$, and $\mathrm{EtOH}(2 \mathrm{~mL})$ to provide acetal $7 \mathrm{~b}$ as a colorless oil $(1.34 \mathrm{~g}, 20 \%)$ :

${ }^{1} \mathrm{H}$ NMR $\left(400 \mathrm{MHz}, \mathrm{CDCl}_{3}\right) \delta 8.06(\mathrm{~d}, J=8.9,2 \mathrm{H}), 7.37(\mathrm{~d}, J=8.1,2 \mathrm{H}), 7.15(\mathrm{~d}, J=8.1,2 \mathrm{H}), 6.93$ (d, $J=8.9,2 \mathrm{H}), 5.97(\mathrm{~d}, J=6.1,1 \mathrm{H}), 4.78(\mathrm{~d}, J=6.1,2 \mathrm{H}), 3.87(\mathrm{~s}, 3 \mathrm{H}), 3.61-3.79(\mathrm{~m}, 3 \mathrm{H}), 3.39-$ $3.48(\mathrm{~m}, 1 \mathrm{H}), 2.33(\mathrm{~s}, 3 \mathrm{H}), 1.19(\mathrm{t}, J=7.1,3 \mathrm{H}), 1.09(\mathrm{t}, J=7.1,3 \mathrm{H})$;

${ }^{13} \mathrm{C}$ NMR (100 MHz, $\left.\mathrm{CDCl}_{3}\right) \delta 165.3(\mathrm{C}), 163.5(\mathrm{C}), 137.9(\mathrm{C}), 134.2(\mathrm{C}), 131.9(\mathrm{CH}), 129.0(\mathrm{CH})$, $127.8(\mathrm{CH}), 122.9(\mathrm{C}), 113.7(\mathrm{CH}), 103.4(\mathrm{CH}), 75.3(\mathrm{CH}), 64.1\left(\mathrm{CH}_{2}\right), 62.8\left(\mathrm{CH}_{2}\right), 55.6\left(\mathrm{CH}_{3}\right)$, $21.4\left(\mathrm{CH}_{3}\right), 15.5\left(\mathrm{CH}_{3}\right), 15.2\left(\mathrm{CH}_{3}\right)$;

IR (ATR) 2973, 1713, 1606, 1097, $768 \mathrm{~cm}^{-1}$;

HRMS (APCl) m/z: [[M + H] $\left.-\mathrm{C}_{2} \mathrm{H}_{6} \mathrm{O}\right]^{+}$Calcd for $\mathrm{C}_{19} \mathrm{H}_{21} \mathrm{O}_{4} 313.1434$; Found 313.1443. 
<smiles>C=CC(OC)c1ccc(C)cc1</smiles>

S27

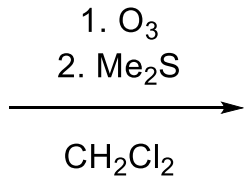

1. $\mathrm{O}_{3}$<smiles>COC(C=O)c1ccc(C)cc1</smiles>

S36<smiles>CCOCCO</smiles><smiles>CCOC(OC)C(OC)c1ccc(C)cc1</smiles>

1-(2,2-Diethoxy-1-methoxyethyl)-4-methylbenzene (6). Following the general procedure for ozonolysis, ester $\mathbf{S} 27(0.88 \mathrm{~g}, 5.0 \mathrm{mmol})$ was treated with $\mathrm{O}_{3}$ to provide aldehyde $\mathbf{S} 36$ as a yellow oil. Following the general procedure for acetal formation, aldehyde $\mathbf{S} 36(0.88 \mathrm{~g}, 5.0 \mathrm{mmol})$ was mixed with triethyl orthoformate $(8.32 \mathrm{~mL}, 15.0 \mathrm{mmol}), p$-toluenesulfonic acid $(0.482 \mathrm{~g}, 2.50$ $\mathrm{mmol})$, and $\mathrm{EtOH}(0.5 \mathrm{~mL})$ to provide acetal 6 as a colorless oil $(0.425 \mathrm{~g}, 35 \%)$ :

${ }^{1} \mathrm{H}$ NMR $\left(400 \mathrm{MHz}, \mathrm{CDCl}_{3}\right) \delta 7.24(\mathrm{~d}, J=8.2,2 \mathrm{H}), 7.16(\mathrm{~d}, J=8.2,2 \mathrm{H}), 4.47(\mathrm{~d}, J=6.2,1 \mathrm{H}), 4.15$ $(\mathrm{d}, J=6.2,1 \mathrm{H}), 3.70-3.78(\mathrm{~m}, 1 \mathrm{H}), 3.52-3.66(\mathrm{~m}, 2 \mathrm{H}), 3.27(\mathrm{~s}, 3 \mathrm{H}), 3.19-3.25(\mathrm{~m}, 1 \mathrm{H}), 2.37(\mathrm{~s}$, $3 \mathrm{H}), 1.26$ (t, $J=7.4,3 \mathrm{H}), 0.99(\mathrm{t}, J=7.4,3 \mathrm{H})$;

${ }^{13} \mathrm{C}$ NMR (100 MHz, $\left.\mathrm{CDCl}_{3}\right) \delta 137.5(\mathrm{C}), 135.4(\mathrm{C}), 128.9(\mathrm{CH}), 128.1(\mathrm{CH}), 104.7(\mathrm{CH}), 84.5$ $(\mathrm{CH}), 63.9\left(\mathrm{CH}_{2}\right), 62.6\left(\mathrm{CH}_{2}\right), 57.1\left(\mathrm{CH}_{3}\right), 21.3\left(\mathrm{CH}_{3}\right), 15.5\left(\mathrm{CH}_{3}\right), 15.2\left(\mathrm{CH}_{3}\right)$;

IR (ATR) 2975, 1103, 1052, 813, $771 \mathrm{~cm}^{-1}$;

HRMS (APCl) m/z: [[M + H] $\left.-\mathrm{C}_{3} \mathrm{H}_{6} \mathrm{O}\right]^{+}$Calcd for $\mathrm{C}_{11} \mathrm{H}_{15} \mathrm{O}_{2}$ 179.1067; Found 179.1069.

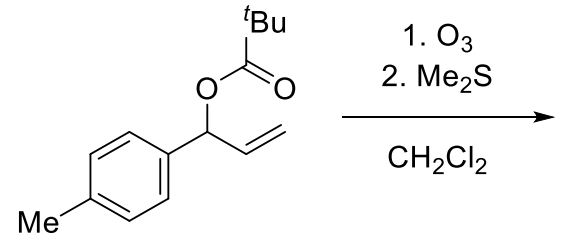

S23<smiles>Cc1ccc(C(OC(=O)CC(C)(C)C)C(=O)C(C)(C)C)cc1</smiles>

S32

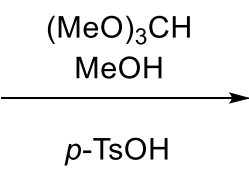<smiles>COC(Cc1ccc(C)cc1)OC</smiles>

18

2,2-Dimethoxy-1-(p-tolyl)ethyl pivaloate (18). Following the general procedure for ozonolysis, ester S23 (0.497 g, $2.14 \mathrm{mmol}$ ) was treated with $\mathrm{O}_{3}$ to provide aldehyde $\mathbf{S} 32$ as a yellow oil. Following the general procedure for acetal formation, aldehyde $\mathbf{S} 32(0.497 \mathrm{~g}, 2.14 \mathrm{mmol})$ was mixed with trimethyl orthoformate $(2.34 \mathrm{~mL}, 21.4 \mathrm{mmol}), p$-toluenesulfonic acid $(0.204 \mathrm{~g}, 1.07$ $\mathrm{mmol})$, and $\mathrm{MeOH}(0.5 \mathrm{~mL})$ to provide acetal 18 as a colorless oil $(0.429 \mathrm{~g}, 71 \%)$ :

${ }^{1} \mathrm{H}$ NMR $\left(400 \mathrm{MHz}, \mathrm{CDCl}_{3}\right) \delta 7.28(\mathrm{~d}, J=8.1,2 \mathrm{H}), 7.17(\mathrm{~d}, J=8.1,2 \mathrm{H}), 5.77(\mathrm{~d}, J=6.9,1 \mathrm{H}), 4.5$ $(\mathrm{d}, J=6.9,1 \mathrm{H}), 3.43(\mathrm{~s}, 3 \mathrm{H}), 3.32(\mathrm{~s}, 3 \mathrm{H}), 2.36(\mathrm{~s}, 3 \mathrm{H}), 1.24(\mathrm{~s}, 9 \mathrm{H})$;

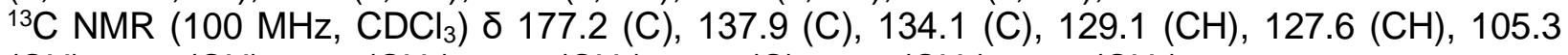
$(\mathrm{CH}), 74.0(\mathrm{CH}), 55.7\left(\mathrm{CH}_{3}\right), 54.0\left(\mathrm{CH}_{3}\right), 38.9(\mathrm{C}), 27.2\left(\mathrm{CH}_{3}\right), 21.4\left(\mathrm{CH}_{3}\right)$;

IR (ATR) 2973, 1713, 1606, 1251, $790 \mathrm{~cm}^{-1}$;

HRMS (ESI): m/z: [M + Na] ${ }^{+}$Calcd for $\mathrm{C}_{16} \mathrm{H}_{24} \mathrm{NaO}_{4}$ 303.1567; Found 303.1572.<smiles>CCOC(OCC)C(OC(=O)CC(C)(C)C)c1ccc(C)cc1</smiles><smiles>Cc1ccc(C(OC(=O)CC(C)(C)C)C(Cc2ccccc2)OCc2ccccc2)cc1</smiles>

2,2-Bis(benzyloxy)-1-(p-tolyl)ethyl pivaloate (19). To a $-45{ }^{\circ} \mathrm{C}$ solution of acetal $4 \mathrm{a}(0.25 \mathrm{~g}$, $0.80 \mathrm{mmol})$ in $\mathrm{CH}_{2} \mathrm{Cl}_{2}(8 \mathrm{~mL}, 0.1 \mathrm{M})$ was added $\mathrm{BnOH}(0.421 \mathrm{~mL}, 4.05 \mathrm{mmol})$. After $30 \mathrm{~min}$. trimethylsilyl trifluoromethanesulfonate $(0.30 \mathrm{~mL}, 1.6 \mathrm{mmol})$ was added. After $16 \mathrm{~h}$, a 1:1:1 (v/v/v) mixture of $\mathrm{CH}_{2} \mathrm{Cl}_{2}: \mathrm{EtOH}: \mathrm{Et}_{3} \mathrm{~N}$ was added, and the reaction mixture was warmed to room temperature over $30 \mathrm{~min}$. The reaction mixture was then washed with $5 \% \mathrm{HCl}(5 \mathrm{~mL})$ and the 
aqueous layer was extracted with $\mathrm{Et}_{2} \mathrm{O}(2 \times 10 \mathrm{~mL})$. The combined organic layers were washed with saturated $\mathrm{NaHCO}_{3}(10 \mathrm{~mL})$ and brine $(10 \mathrm{~mL})$, dried over $\mathrm{Na}_{2} \mathrm{SO}_{4}$, filtered through cotton, and concentrated in vacuo. The residue was purified by flash column chromatography using deactivated silica (pentanes to 3:97 $\mathrm{Et}_{2} \mathrm{O}$ :hexanes) to provide benzyl acetal 19 as a colorless oil $(0.255 \mathrm{~g}, 73 \%)$ containing traces of benzyl alcohol which could not be removed:

${ }^{1} \mathrm{H}$ NMR $\left(400 \mathrm{MHz}, \mathrm{CDCl}_{3}\right) \delta$ 7.27-7.34 (m, 10H), $7.15(\mathrm{~d}, J=8,2 \mathrm{H}), 7.01(\mathrm{~m}, 2 \mathrm{H}), 5.91(\mathrm{~d}, J=$ 7.8,1H), $4.86(\mathrm{~d}, J=6.4,1 \mathrm{H}), 4.67(\mathrm{~s}, 2 \mathrm{H}), 4.57(\mathrm{~d}, J=12.3,1 \mathrm{H}), 4.40(\mathrm{~d}, J=12.9,1 \mathrm{H}), 2.37(\mathrm{~s}$, $3 \mathrm{H}), 1.19(\mathrm{~s}, 9 \mathrm{H})$;

${ }^{13} \mathrm{C}$ NMR (100 MHz, CDCl ${ }_{3}$ ) 177.2 (C), 141.0 (C), 137.9 (C), 137.5 (C), 134.2 (C), 129.0 (, CH), $128.5(\mathrm{CH}), 128.4(\mathrm{CH}), 127.9(\mathrm{CH}), 127.82(\mathrm{CH}), 127.77(\mathrm{CH}), 127.76(\mathrm{CH}), 127.69(\mathrm{CH}), 102.1$ $(\mathrm{CH}), 74.6(\mathrm{CH}), 69.7\left(\mathrm{CH}_{2}\right), 67.9\left(\mathrm{CH}_{2}\right), 38.9(\mathrm{C}), 27.2\left(\mathrm{CH}_{3}\right), 21.4\left(\mathrm{CH}_{3}\right)$;

IR (ATR) 2972, 2932, 1734, 1153, $1027 \mathrm{~cm}^{-1}$;

HRMS (ESI) m/z: [[M + H] - $\left.\mathrm{C}_{5} \mathrm{H}_{10} \mathrm{O}_{2}\right]^{+}$Calcd for $\mathrm{C}_{23} \mathrm{H}_{23} \mathrm{O}_{2}$ 331.1893; Found 331.1883.

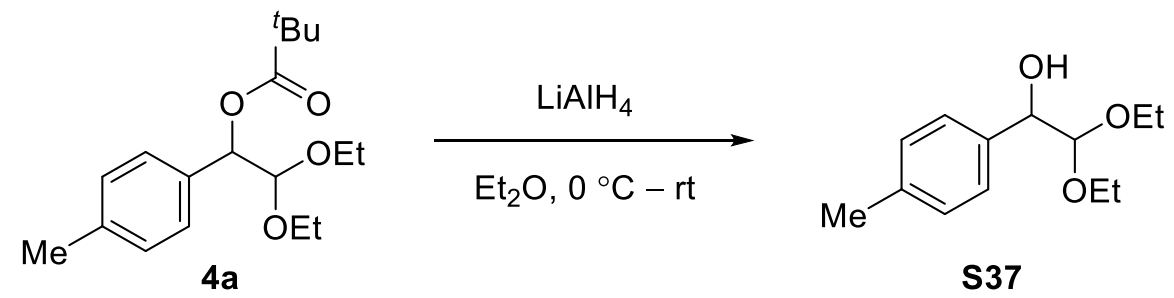

2,2-Diethoxy-1-(p-tolyl)ethan-1-ol (S37). This procedure was adapted from that reported by Rein et. al. ${ }^{10}$ To a $0{ }^{\circ} \mathrm{C}$ solution of acetal $4 \mathrm{a}(0.795 \mathrm{~g}, 2.50 \mathrm{mmol})$ in $\mathrm{Et}_{2} \mathrm{O}(25 \mathrm{~mL}, 0.10 \mathrm{M})$ was added $\mathrm{LiAlH}_{4}(0.142 \mathrm{~g}, 3.75 \mathrm{mmol})$. The reaction mixture was warmed to room temperature over $1 \mathrm{hr}$. After $1 \mathrm{~h}$, the reaction mixture was diluted with EtOAc $(10 \mathrm{~mL})$ and a 1:1 solution (v/v) of $\mathrm{H}_{2} \mathrm{O}: \mathrm{MeOH}(20 \mathrm{~mL})$ was added. The mixture was stirred for $5 \mathrm{~h}$ until it turned clear, and then the aqueous layer was extracted with EtOAc $(2 \times 20 \mathrm{~mL})$. The combined organic layers were washed with $\mathrm{H}_{2} \mathrm{O}(30 \mathrm{~mL})$, then brine $(20 \mathrm{~mL})$, dried over $\mathrm{Na}_{2} \mathrm{SO}_{4}$, filtered through cotton, and concentrated in vacuo. The residue was purified by flash column chromatography (10:90 EtOAc:hexanes) to provide alcohol S37 as a colorless oil $(0.515 \mathrm{~g}, 92 \%)$ :

${ }^{1} \mathrm{H}$ NMR $\left(400 \mathrm{MHz}, \mathrm{CDCl}_{3}\right) \delta 7.32(\mathrm{~d}, J=7.9,2 \mathrm{H}), 7.16(\mathrm{~d}, J=7.9,2 \mathrm{H}), 4.57(\mathrm{dd}, J=6.1,1.8$, $1 \mathrm{H}), 4.38(\mathrm{~d}, J=6.5,1 \mathrm{H}), 3.78-3.86(\mathrm{~m}, 1 \mathrm{H}), 3.52-3.63(\mathrm{~m}, 2 \mathrm{H}), 3.22-3.19(\mathrm{~m}, 1 \mathrm{H}), 2.71(\mathrm{~d}, J=$ $1.8,1 \mathrm{H}), 2.35(\mathrm{~s}, 3 \mathrm{H}), 1.27(\mathrm{t}, J=6.7,3 \mathrm{H}), 1.06(\mathrm{t}, J=6.7,3 \mathrm{H})$;

${ }^{13} \mathrm{C}$ NMR $\left(100 \mathrm{MHz}_{\mathrm{CDCl}}\right) \delta 137.5(\mathrm{C}), 136.6(\mathrm{C}), 128.9(\mathrm{CH}), 127.2(\mathrm{CH}), 106.2(\mathrm{CH}), 74.6$ $(\mathrm{CH}), 64.6\left(\mathrm{CH}_{2}\right), 63.7\left(\mathrm{CH}_{2}\right), 21.3\left(\mathrm{CH}_{3}\right), 15.5\left(\mathrm{CH}_{3}\right), 15.3\left(\mathrm{CH}_{3}\right)$;

IR (ATR) 3328, 2973, 2883, 1046, $904 \mathrm{~cm}^{-1}$;

HRMS (APCI) m/z: [[M + H] $\left.-\mathrm{C}_{2} \mathrm{H}_{6} \mathrm{O}\right]^{+}$Calcd for $\mathrm{C}_{11} \mathrm{H}_{15} \mathrm{O}_{2}$ 179.1067; Found 179.1063 .<smiles>CCOC(O)C(O)c1ccc(C)cc1</smiles>

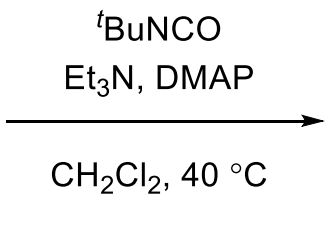<smiles>CCOC(OCC)C(OC(=O)NCC(C)C)c1ccc(C)cc1</smiles>

2,2-Diethoxy-1-(p-tolyl)ethyl tert-butylcarbamate (7d). Following the general procedure for esterification, a solution of alcohol $\mathrm{S} 37(0.6 \mathrm{~g}, 3 \mathrm{mmol})$ in $\mathrm{CH}_{2} \mathrm{Cl}_{2}(3 \mathrm{~mL})$ was mixed $\mathrm{Et}_{3} \mathrm{~N}(0.84$ $\mathrm{mL}, 6.0 \mathrm{mmol})$, dimethylaminopyridine $(0.04 \mathrm{~g}, 0.3 \mathrm{mmol})$, and tert-butyl isocyanate $(0.6 \mathrm{~mL}, 6$ $\mathrm{mmol}$ ) at reflux $\left(40^{\circ} \mathrm{C}\right.$, oil bath) for $24 \mathrm{~h}$ to provide acetal $7 \mathrm{~d}$ as a colorless oil $(0.625 \mathrm{~g}, 65 \%)$ : 
${ }^{1} \mathrm{H} \mathrm{NMR}\left(400 \mathrm{MHz}, \mathrm{CDCl}_{3}\right) \delta 7.26(\mathrm{~d}, J=7.8,2 \mathrm{H}), 7.13(\mathrm{~d}, J=7.8,2 \mathrm{H}), 5.72(\mathrm{~d}, J=5.5,1 \mathrm{H}), 5.32$ $(\mathrm{s}, 0.6 \mathrm{H}), 4.62(\mathrm{~d}, J=1.4,1 \mathrm{H}), 3.59-4.63(\mathrm{~m}, 3 \mathrm{H}), 3.33-3.58(\mathrm{~m}, 1 \mathrm{H}), 2.34(\mathrm{~s}, 3 \mathrm{H}), 1.22(\mathrm{~s}, 9 \mathrm{H})$, $1.19-1.21(\mathrm{t}, \mathrm{J}=6.4,3 \mathrm{H})$;

${ }^{13} \mathrm{C}$ NMR (100 MHz, $\left.\mathrm{CDCl}_{3}\right) \delta 176.9(\mathrm{C}), 137.6(\mathrm{C}), 134.2(\mathrm{C}), 136.4(\mathrm{CH}), 128.8(\mathrm{CH}), 127.5$ $(\mathrm{CH}), 74.5(\mathrm{CH}), 63.8\left(\mathrm{CH}_{2}\right), 62.1\left(\mathrm{CH}_{2}\right), 38.8(\mathrm{C}), 27.1\left(\mathrm{CH}_{3}\right), 21.2\left(\mathrm{CH}_{3}\right), 15.3\left(\mathrm{CH}_{3}\right), 15.0\left(\mathrm{CH}_{3}\right)$; IR (ATR) 2974, 1731, 1479, 1278, $1117 \mathrm{~cm}^{-1}$;

HRMS (APCl) m/z: [[M + H] - $\left.\mathrm{C}_{7} \mathrm{H}_{15} \mathrm{NO}_{2}\right]^{+}$Calcd for $\mathrm{C}_{11} \mathrm{H}_{15} \mathrm{O}_{2}$ 179.1067; Found 179.1067.<smiles>CCOC(OCC)C(O)c1ccc(C)cc1</smiles><smiles>CCCCCN(CC)CC</smiles><smiles>CCOC(OCC)C(OC(=O)c1ccccc1)c1ccc(C)cc1</smiles>

7c

2,2-Diethoxy-1-(p-tolyl)ethyl benzoate (7c). Following the general procedure for esterification, a solution of alcohol $\mathrm{S} 37(0.254 \mathrm{~g}, 1.13 \mathrm{mmol})$ in $\mathrm{CH}_{2} \mathrm{Cl}_{2}(2.5 \mathrm{~mL})$ was mixed with $\mathrm{Et}_{3} \mathrm{~N}(0.32 \mathrm{~mL}$, $2.3 \mathrm{mmol})$, dimethylaminopyridine $(0.14 \mathrm{~g}, 0.11 \mathrm{mmol})$, and benzoyl chloride $(0.264,2.27 \mathrm{mmol})$ to give ester $7 \mathrm{c}$ as a colorless oil $(0.338 \mathrm{~g}, 91 \%)$ :

${ }^{1} \mathrm{H}$ NMR $\left(400 \mathrm{MHz}, \mathrm{CDCl}_{3}\right) \delta 8.10(\mathrm{~d}, J=8.3,2 \mathrm{H}), 7.57(\mathrm{t}, J=7.9,1 \mathrm{H}), 7.45(\mathrm{t}, J=7.9,2 \mathrm{H}), 7.38$ $(\mathrm{d}, J=7.9,2 \mathrm{H}), 7.15(\mathrm{~d}, J=7.9,2 \mathrm{H}), 6.00(\mathrm{~d}, J=5.7,1 \mathrm{H}), 4.79(\mathrm{~d}, J=5.7,1 \mathrm{H}), 3.72-3.80(\mathrm{~m}$, $1 \mathrm{H}), 3.62-3.74(\mathrm{~m}, 2 \mathrm{H}), 3.39-3.48(\mathrm{~m}, 1 \mathrm{H}), 2.34(\mathrm{~s}, 3 \mathrm{H}), 1.19(\mathrm{t}, J=6.9,3 \mathrm{H}), 1.09(\mathrm{t}, J=7.1,3 \mathrm{H})$; ${ }^{13} \mathrm{C} \mathrm{NMR}\left(100 \mathrm{MHz}, \mathrm{CDCl}_{3}\right) \delta 165.5(\mathrm{C}), 138.0(\mathrm{C}), 134.1(\mathrm{C}), 133.1(\mathrm{C}), 130.6(\mathrm{CH}), 129.9(\mathrm{CH})$, $128.9(\mathrm{CH}), 128.5(\mathrm{CH}), 127.9(\mathrm{CH}), 103.4(\mathrm{CH}), 75.7(\mathrm{CH}), 64.1\left(\mathrm{CH}_{2}\right), 62.8\left(\mathrm{CH}_{2}\right), 21.4\left(\mathrm{CH}_{3}\right)$, $15.5\left(\mathrm{CH}_{3}\right), 15.2\left(\mathrm{CH}_{3}\right)$;

IR (ATR) 1787, 1269, 1210, 996, $814 \mathrm{~cm}^{-1}$;

HRMS (APCI) m/z: [[M + H] $\left.-\mathrm{C}_{2} \mathrm{H}_{6} \mathrm{O}\right)^{+}$Calcd for $\mathrm{C}_{19} \mathrm{H}_{19} \mathrm{O}_{3}{ }^{+}$283.1329; Found 283.1327.

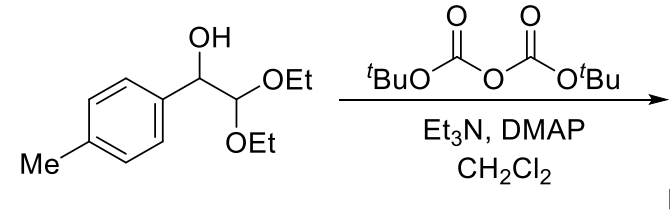

S37<smiles>CCOC(=O)OC(c1ccc(C)cc1)C(CC)OCC</smiles>

12<smiles>CCO[C@@H]1OC(=O)O[C@H]1c1ccc(C)cc1</smiles>

14<smiles>CCO[C@@H]1OC(=O)O[C@H]1c1ccc(C)cc1</smiles>

epi-14

tert-Butyl (2,2-diethoxy-1-(p-tolyl)ethyl) carbonate (12), $\left(4 R^{\star}, 5 R^{\star}\right)-4-e t h o x y-5-(p$-tolyl)-1,3dioxolan-2-one (14), $\left(4 R^{\star}, 5 S^{\star}\right)$-4-ethoxy-5-(p-tolyl)-1,3-dioxolan-2-one (epi-14). Following the general procedure for esterification, a solution of alcohol $\mathbf{S} 37(0.26 \mathrm{~g}, 1.2 \mathrm{mmol})$ in $\mathrm{CH}_{2} \mathrm{Cl}_{2}(2.5$ $\mathrm{mL})$ was mixed with $\mathrm{Et}_{3} \mathrm{~N}(0.335 \mathrm{~mL}, 2.40 \mathrm{mmol})$, dimethylaminopyridine $(0.015 \mathrm{~g}, 0.12 \mathrm{mmol})$, and di-tert-butyl dicarbonate $(0.225 \mathrm{~g}, 2.40 \mathrm{mmol})$. Purification by column chromatography using deactivated silica (3:97 EtOAc:hexanes) provided carbonate 12 as a colorless oil $(0.257 \mathrm{~g}, 66 \%)$ and cyclic carbonate 14 as a mixture of diastereomers (14:epi-14 =96:4) and as a colorless oil $(0.015 \mathrm{~g}, 5 \%)$. On the other hand, purification of carbonate 12 using $\mathrm{SiO}_{2}$ afforded carbonate 12 and cyclic carbonate 14 in a 37:63 mixture and cyclic carbonate 14 mixture of diastereomers (14:epi-14 = 76:24). 
Carbonate 12: ${ }^{1} \mathrm{H}$ NMR (400 MHz, $\left.\mathrm{CDCl}_{3}\right) \delta 7.30(\mathrm{~d}, J=7.8,2 \mathrm{H}), 7.15(\mathrm{~d}, J=7.8,2 \mathrm{H}), 5.54(\mathrm{~d}, J$ $=6.1,1 \mathrm{H}), 4.61(\mathrm{~d}, J=6.9,1 \mathrm{H}), 3.72-3.80(\mathrm{~m}, 1 \mathrm{H}), 3.56-3.64(\mathrm{~m}, 2 \mathrm{H}), 3.29-3.37(\mathrm{~m}, 1 \mathrm{H}), 2.34$ (s, 3H), $1.46(\mathrm{~s}, 9 \mathrm{H}), 1.23(\mathrm{t}, J=6.9,3 \mathrm{H}), 1.03(\mathrm{t}, J=6.9,3 \mathrm{H})$;

${ }^{13} \mathrm{C}$ NMR (100 MHz, CDCl $)$ ס $152.9(\mathrm{C}), 137.9(\mathrm{C}), 133.9(\mathrm{C}), 128.9(\mathrm{CH}), 127.8(\mathrm{CH}), 103.6$ $(\mathrm{CH}), 82.3(\mathrm{C}), 77.9(\mathrm{CH}), 63.8\left(\mathrm{CH}_{2}\right), 63.3\left(\mathrm{CH}_{2}\right), 27.9\left(\mathrm{CH}_{3}\right), 21.5\left(\mathrm{CH}_{3}\right), 15.4\left(\mathrm{CH}_{3}\right), 15.1\left(\mathrm{CH}_{3}\right)$; IR (ATR) 2976, 1743, 1371, 1063, $787 \mathrm{~cm}^{-1}$;

HRMS (APCl) m/z: [[M + 2H] - $\left.\mathrm{C}_{7} \mathrm{H}_{15} \mathrm{O}_{3}\right]^{+}$Calcd for $\mathrm{C}_{11} \mathrm{H}_{15} \mathrm{O}_{2}$ 179.1067; Found 179.1063.

Cyclic carbonates 14 and epi-14: ${ }^{1} \mathrm{H}$ NMR $\left(400 \mathrm{MHz}, \mathrm{CDCl}_{3}\right) \delta 7.26(\mathrm{~s}, 0.12 \mathrm{H}), 7.24$ (d, $J=7.8$, $2.12 \mathrm{H}), 7.20(\mathrm{~d}, J=7.8,2 \mathrm{H}), 5.69(\mathrm{~d}, J=7.2,0.06 \mathrm{H}), 5.58(\mathrm{~d}, J=5.3,0.06 \mathrm{H}), 5.42(\mathrm{~d}, J=2.6$, $1 \mathrm{H}), 5.27(\mathrm{~d}, J=2.6,1 \mathrm{H}), 3.89-3.98(\mathrm{~m}, 1 \mathrm{H}), 3.81-3.84(\mathrm{~m}, 0.12 \mathrm{H}), 3.64-3.71(\mathrm{~m}, 1 \mathrm{H}), 2.37(\mathrm{~s}$, $3 \mathrm{H}), 2.34(\mathrm{~s}, 0.18 \mathrm{H}), 1.29(\mathrm{t}, J=6.8,3 \mathrm{H}), 1.10(\mathrm{t}, J=7.3,0.18 \mathrm{H})$;

Peaks in ${ }^{13} \mathrm{C}$ NMR $\left(100 \mathrm{MHz}, \mathrm{CDCl}_{3}\right)$ attributed to the major diastereomer, 14: $\delta 153.9(\mathrm{C}), 139.9$ $(\mathrm{C}), 131.7(\mathrm{C}), 129.9(\mathrm{CH}), 125.7(\mathrm{CH}), 105.9(\mathrm{CH}), 83.4(\mathrm{CH}), 66.2\left(\mathrm{CH}_{2}\right), 21.3\left(\mathrm{CH}_{3}\right), 14.9\left(\mathrm{CH}_{3}\right)$; Peaks in ${ }^{13} \mathrm{C}$ NMR $\left(100 \mathrm{MHz}, \mathrm{CDCl}_{3}\right.$ ) attributed to the minor diastereomer, epi-14: $\delta 154.1$ (C), $139.3(\mathrm{C}), 131.4(\mathrm{C}), 129.0(\mathrm{CH}), 127.6(\mathrm{CH}), 101.2(\mathrm{CH}), 81.8(\mathrm{CH}), 66.1\left(\mathrm{CH}_{2}\right), 21.4\left(\mathrm{CH}_{3}\right), 14.7$ $\left(\mathrm{CH}_{3}\right)$;

IR (ATR) 2981, 1809, 1120, 904, $723 \mathrm{~cm}^{-1}$;

HRMS (APCl) m/z: [[M + H] - $\left.\mathrm{CO}_{2}\right]^{+}$Calcd for $\mathrm{C}_{11} \mathrm{H}_{15} \mathrm{O}_{2}$ 179.1067; Found 179.1064. 


\section{Acetal substitution reactions}

General procedure for acetal substitutions. To a cooled $\left(-45^{\circ} \mathrm{C}\right)$ solution of acetal (1 equiv) in solvent $(0.1 \mathrm{M})$ and activated molecular sieves (about $0.1 \mathrm{~g}$ per $\mathrm{mmol}$ ) was added nucleophile (4 equiv). After 30 min, trimethylsilyl trifluoromethanesulfonate (2 equiv) was added. After $16 \mathrm{~h}, \mathrm{a}$ $1: 1: 1(\mathrm{v} / \mathrm{v} / \mathrm{v})$ mixture of $\mathrm{CH}_{2} \mathrm{Cl}_{2}: \mathrm{EtOH}: \mathrm{Et}_{3} \mathrm{~N}(5 \mathrm{~mL}$ per mmol) was added, and then the reaction mixture was warmed to room temperature over $30 \mathrm{~min}$. The reaction mixture was washed with $5 \% \mathrm{HCl}\left(5 \mathrm{~mL}\right.$ per mmol) and the aqueous layer was extracted with $\mathrm{Et}_{2} \mathrm{O}(2 \times 10 \mathrm{~mL}$ per mmol). The combined organic layers were washed with a saturated solution of $\mathrm{NaHCO}_{3}(10 \mathrm{~mL}$ per $\mathrm{mmol}$ ), then brine (10 $\mathrm{mL}$ per $\mathrm{mmol}$ ), dried over $\mathrm{Na}_{2} \mathrm{SO}_{4}$, filtered through cotton, and concentrated in vacuo. The diastereomeric and product ratios were determined by NMR analysis $\left({ }^{1} \mathrm{H}\right.$ and $\left.{ }^{13} \mathrm{C}\right)$ of the unpurified mixture. The residue was purified by flash column chromatography (pentanes to 3:97 $\mathrm{Et}_{2} \mathrm{O}$ :hexanes) to provide the product as a colorless oil. Multiple purifications by flash column chromatography were required to remove excess tributylstannyl impurities.<smiles>CCOC(=O)OC(c1ccc(C)cc1)C(OCC)OCC</smiles>

<smiles>CCOC1OC(=O)O[C@H]1c1ccc(C)cc1</smiles><smiles>CCOC1OC(=O)O[C@H]1c1ccc(C)cc1</smiles>

$\left(4 R^{\star}, 5 R^{\star}\right)$-4-Ethoxy-5-( $p$-tolyl)-1,3-dioxolan-2-one (14) and $\left(4 R^{\star}, 5 S^{\star}\right)$-4-ethoxy-5-( $p$-tolyl)1,3-dioxolan-2-one (epi-14). Following the general procedure for acetal substitutions, acetal 12 $(0.050 \mathrm{~g}, 0.15 \mathrm{mmol})$ in $\mathrm{CH}_{2} \mathrm{Cl}_{2}(1.5 \mathrm{~mL})$ was mixed with allyltributylstannane $(0.204 \mathrm{~g}, 0.620$ $\mathrm{mmol})$ and trimethylsilyl trifluoromethanesulfonate $(0.056 \mathrm{~mL}, 0.31 \mathrm{mmol})$ to give cyclic carbonate 14 as a mixture of diastereomers (14:epi-14 = 76:24) and as a colorless oil $(0.003 \mathrm{~g}, 9 \%)$. The product was isolated as a 76:24 mixture of diastereomers and the spectral data obtained are consistent with those reported above.

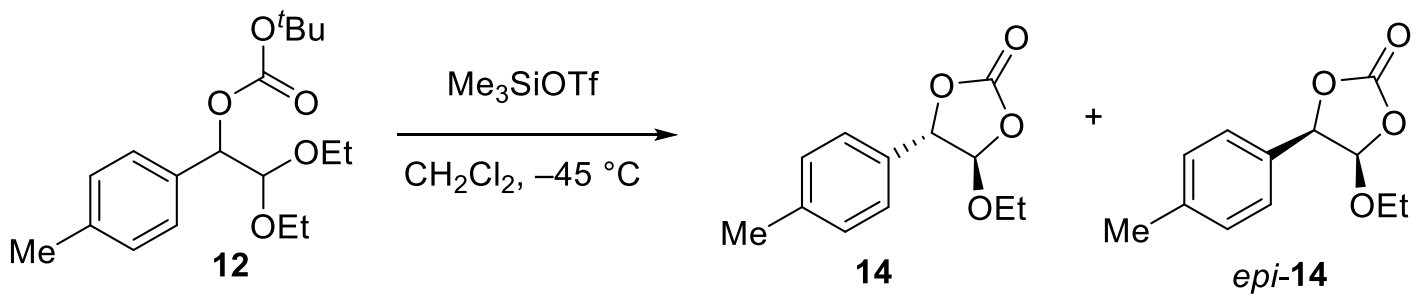

$\left(4 R^{\star}, 5 R^{\star}\right)$-4-Ethoxy-5-(p-tolyl)-1,3-dioxolan-2-one (14) and $\left(4 R^{\star}, 5 S^{\star}\right)$-4-ethoxy-5-(p-tolyl)1,3-dioxolan-2-one (epi-14). Following the general procedure for acetal substitutions, acetal 12 $(0.098 \mathrm{~g}, 0.30 \mathrm{mmol})$ in $\mathrm{CH}_{2} \mathrm{Cl}_{2}(3 \mathrm{~mL})$ was mixed with trimethylsilyl trifluoromethanesulfonate $(0.11 \mathrm{~mL}, 0.58 \mathrm{mmol})$ to give cyclic carbonate 14 as a mixture of diastereomers (14:epi-14=96:4) and as a colorless oil $(0.047 \mathrm{~g}, 71 \%)$. The spectral data obtained are consistent with those reported above.

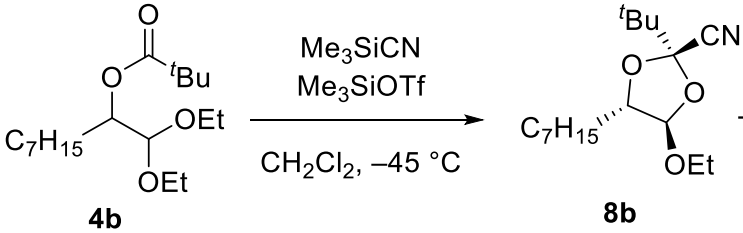

4b
$8 b$<smiles>CCOC1OC([18CH])(C#N)O[C@H]1CC</smiles>

epi-8b<smiles>CCO[C@@H]1O[C@](C)(C(C)(C)C)O[C@H]1[13CH3]</smiles>

epi-8b'

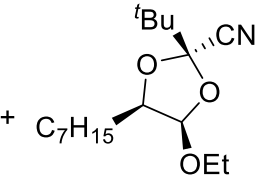

epi-8b" 
$\left(2 R^{\star}, 4 R^{\star}, 5 S^{\star}\right)-2-($ tert-Butyl)-4-ethoxy-5-heptyl-1,3-dioxolane-2-carbonitrile

$(8 b)$, $\left(2 R^{\star}, 4 R^{\star}, 5 R^{\star}\right)$-2-(tert-butyl)-4-ethoxy-5-heptyl-1,3-dioxolane-2-carbonitrile $\left(2 R^{\star}, 4 S^{\star}, 5 R^{\star}\right)$-2-(tert-butyl)-4-ethoxy-5-heptyl-1,3-dioxolane-2-carbonitrile $\left(2 R^{\star}, 4 S^{\star}, 5 S^{\star}\right)$-2-(tert-butyl)-4-ethoxy-5-heptyl-1,3-dioxolane-2-carbonitrile (epi-8b), $\left(e p i-8 b^{\prime}\right)$, and (epi-8b"). Following the general procedure for acetal substitutions, acetal $4 \mathbf{b}(0.158 \mathrm{~g}, 0.5 \mathrm{mmol})$ in $\mathrm{CH}_{2} \mathrm{Cl}_{2}$ $(5 \mathrm{~mL})$ was mixed with trimethylsilyl cyanide $(0.397 \mathrm{~mL}, 2.00 \mathrm{mmol})$ and trimethylsilyl trifluoromethanesulfonate $(0.182 \mathrm{~mL}, 1.00 \mathrm{mmol})$ to provide a 59:22:12:6 mixture of nitriles $\mathbf{8 b}$, epi-8b, epi-8b', and epi-8b" (corresponding to a 73:27 mixture of $\mathbf{8 b}$ :epi-8b, and a 67:33 mixture of epi-8b:epi-8b) as a colorless oil with minor impurities $(0.107 \mathrm{~g}, 72 \%)$ :

${ }^{1} \mathrm{H}$ NMR $\left(400 \mathrm{MHz}_{\mathrm{CDCl}}\right) \delta 5.23(\mathrm{~d}, J=4.5,0.37 \mathrm{H}), 5.15(\mathrm{~d}, J=5.2,0.1 \mathrm{H}), 5.12(\mathrm{~d}, J=5.2$, $0.2 \mathrm{H}), 4.88(\mathrm{~d}, J=4.5,1 \mathrm{H}), 4.15-4.19(\mathrm{~m}, 1 \mathrm{H}), 4.02-4.07(\mathrm{~m}, 0.37 \mathrm{H}), 3.85-3.98(\mathrm{~m}, 2 \mathrm{H}), 3.71-$ $3.82(\mathrm{~m}, 0.3 \mathrm{H}), 3.55-3.61(\mathrm{~m}, 1.4 \mathrm{H}), 1.24-1.31(\mathrm{~m}, 25 \mathrm{H}), 1.08(\mathrm{~s}, 11.6 \mathrm{H}), 1.06(\mathrm{~s}, 3.5 \mathrm{H}), 0.89(\mathrm{t}$, $J=6.6,5 \mathrm{H})$;

Peaks in ${ }^{13} \mathrm{C}$ NMR (100 MHz, $\mathrm{CDCl}_{3}$ ) attributed to 8b: $\delta 118.1(\mathrm{C}), 106.9(\mathrm{C}), 106.6(\mathrm{CH}), 83.6$ $(\mathrm{CH}), 64.7\left(\mathrm{CH}_{2}\right), 37.6(\mathrm{C}), 31.92\left(\mathrm{CH}_{2}\right), 31.7\left(\mathrm{CH}_{2}\right), 29.4\left(\mathrm{CH}_{2}\right), 29.24\left(\mathrm{CH}_{2}\right), 25.4\left(\mathrm{CH}_{2}\right), 23.93$ $\left(\mathrm{CH}_{3}\right), 22.78\left(\mathrm{CH}_{2}\right), 14.2\left(\mathrm{CH}_{3}, \mathrm{CH}_{3}\right.$ as determined by DEPT and HSQC);

Peaks in ${ }^{13} \mathrm{C} \mathrm{NMR}\left(100 \mathrm{MHz}, \mathrm{CDCl}_{3}\right.$ ) attributed to epi-8b: $\delta 117.5(\mathrm{C}), 106.7(\mathrm{C}), 102.1(\mathrm{CH}), 82.9$ $(\mathrm{CH}), 63.9\left(\mathrm{CH}_{2}\right), 38.8(\mathrm{C}), 31.88\left(\mathrm{CH}_{2}\right), 30.5\left(\mathrm{CH}_{2}\right), 29.22\left(\mathrm{CH}_{2}\right), 28.5\left(\mathrm{CH}_{2}\right), 26.0\left(\mathrm{CH}_{2}\right), 23.7$ $\left(\mathrm{CH}_{3}\right), 22.76\left(\mathrm{CH}_{2}\right), 15.2\left(\mathrm{CH}_{3}, \mathrm{CH}_{3}\right.$ as determined by DEPT and HSQC);

Peaks in ${ }^{13} \mathrm{C}$ NMR (100 MHz, $\mathrm{CDCl}_{3}$ ) attributed to epi-8b' and epi-8b": $\delta 118.5$ (C),118.4 (C), $108.8(\mathrm{C}), 108.4(\mathrm{C}), 106.3(\mathrm{CH}), 100.7(\mathrm{CH}), 84.7(\mathrm{CH}), 81.6(\mathrm{CH}), 64.9\left(\mathrm{CH}_{2}\right), 64.1\left(\mathrm{CH}_{2}\right), 38.4$ (C), $37.4(\mathrm{C}), 31.89\left(\mathrm{CH}_{2}\right), 31.8\left(\mathrm{CH}_{2}\right), 31.2\left(\mathrm{CH}_{2}\right), 29.6\left(\mathrm{CH}_{2}\right), 29.32\left(\mathrm{CH}_{2}\right), 29.29\left(\mathrm{CH}_{2}\right), 28.8$ $\left(\mathrm{CH}_{2}\right), 27.4\left(\mathrm{CH}_{2}\right), 25.7\left(\mathrm{CH}_{2}\right), 25.5\left(\mathrm{CH}_{2}\right), 24.1\left(\mathrm{CH}_{3}\right), 23.91\left(\mathrm{CH}_{3}\right), 23.0\left(\mathrm{CH}_{2}\right), 21.3\left(\mathrm{CH}_{2}\right), 15.13$ $\left(\mathrm{CH}_{3}\right), 15.09\left(\mathrm{CH}_{3}\right), 14.96\left(\mathrm{CH}_{3}\right), 14.92\left(\mathrm{CH}_{3}\right)$;

IR (ATR) 2960, 2928, 1146, 1107, $1059 \mathrm{~cm}^{-1}$;

HRMS (APCl) m/z: [[M + H] - CN] ${ }^{+}$Calcd for $\mathrm{C}_{16} \mathrm{H}_{31} \mathrm{O}_{3}$ 271.2268; Found: 271.2322.

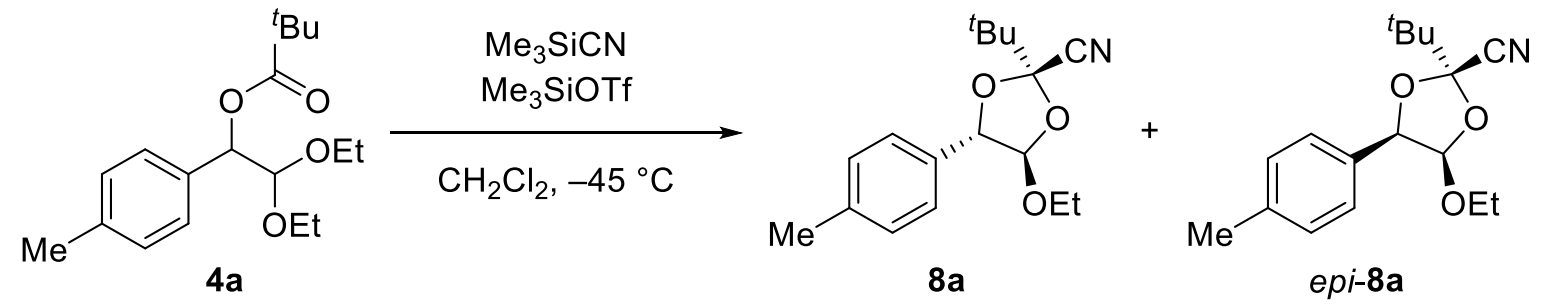

$\left(2 R^{\star}, 4 R^{\star}, 5 R^{\star}\right)-2$-(tert-Butyl)-4-ethoxy-5-(p-tolyl)-1,3-dioxolane-2-carbonitrile $\left(2 R^{\star}, 4 R^{\star}, 5 S^{\star}\right)-2-($ tert-butyl)-4-ethoxy-5-( $p$-tolyl)-1,3-dioxolane-2-carbonitrile

(8a) and (epi-8a). Following the general procedure for acetal substitutions, acetal $4 a(0.154 \mathrm{~g}, 0.500 \mathrm{mmol})$ in $\mathrm{CH}_{2} \mathrm{Cl}_{2}(5 \mathrm{~mL})$ was mixed with trimethylsilyl cyanide $(0.25 \mathrm{~mL}, 2.0 \mathrm{mmol})$ and trimethylsilyl trifluoromethanesulfonate $(0.17 \mathrm{~mL}, 1.0 \mathrm{mmol})$ to provide nitrile $8 \mathbf{a}$ as a mixture of diastereomers (8a:epi-8a $=97: 3)$ and as a clear yellow oil (0.125 g, 86\%). Nitrile $8 \mathbf{a}$ was isolated and characterized as a single diastereomer:

${ }^{1} \mathrm{H} \mathrm{NMR}\left(400 \mathrm{MHz}, \mathrm{CDCl}_{3}\right) \delta 7.26(\mathrm{~d}, J=8.3,2 \mathrm{H}), 7.20(\mathrm{~d}, J=8.3,2 \mathrm{H}), 5.12(\mathrm{~d}, J=4.5,1 \mathrm{H}), 5.08$ (d, $J=4.2,1 \mathrm{H}), 3.87-3.95(\mathrm{~m}, 1 \mathrm{H}), 3.55-3.63(\mathrm{~m}, 1 \mathrm{H}), 2.37(\mathrm{~s}, 3 \mathrm{H}), 1.29(\mathrm{t}, J=7.2,3 \mathrm{H}), 1.19(\mathrm{~s}$, 9H);

${ }^{13} \mathrm{C}$ NMR $\left(100 \mathrm{MHz} \mathrm{CDCl}_{3}\right) \delta 138.9(\mathrm{C}), 132.8(\mathrm{C}), 129.5(\mathrm{CH}), 126.7(\mathrm{CH}), 117.2(\mathrm{C}), 108.2(\mathrm{C})$, $107.2(\mathrm{CH}), 84.9(\mathrm{CH}), 65.3\left(\mathrm{CH}_{2}\right), 37.8(\mathrm{C}), 24.1\left(\mathrm{CH}_{3}\right), 21.3\left(\mathrm{CH}_{3}\right), 15.2\left(\mathrm{CH}_{3}\right)$;

IR (ATR) 2978, 1483, 1139, 1060, $815 \mathrm{~cm}^{-1}$;

HRMS (APCI) m/z: [[M + H] - HCN]+ Calcd for $\mathrm{C}_{16} \mathrm{H}_{23} \mathrm{O}_{3}$ 263.1642; Found 263.1636. 
<smiles>CCO[C@@H]1O[C@](C)(C(C)(C)C)O[C@H]1c1ccc(C)cc1</smiles>

$8 \mathbf{a}$

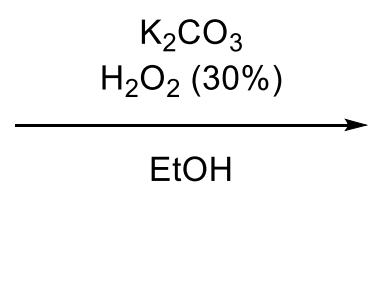<smiles>CCO[C@@H]1O[C@](CC(C)C)(C(N)=O)O[C@H]1c1ccc(C)cc1</smiles>

S38

$\left(2 R^{\star}, 4 R^{\star}, 5 S^{\star}\right)-2$-(tert-Butyl)-4-ethoxy-5-( $p$-tolyl)-1,3-dioxolane-2-carboxamide (S38). This procedure was adapted from that reported by Ao et. al. ${ }^{9}$ To a solution of nitrile $8 \mathbf{a}(0.03 \mathrm{~g}, 0.1$ $\mathrm{mmol})$ in $\mathrm{EtOH}$ was added $\mathrm{K}_{2} \mathrm{CO}_{3}\left(0.031 \mathrm{~g}\right.$ in $0.40 \mathrm{~mL}$ of $\mathrm{H}_{2} \mathrm{O}, 0.34 \mathrm{mmol}, 0.80 \mathrm{M}$ solution). $\mathrm{H}_{2} \mathrm{O}_{2}$ $\left(0.43 \mathrm{~mL}, 0.34 \mathrm{mmol}, 30 \%\right.$ solution in $\mathrm{H}_{2} \mathrm{O}$ ) was then added dropwise over $30 \mathrm{~min}$. After 36 hours, the reaction mixture was diluted with a saturated solution of $\mathrm{Na}_{2} \mathrm{~S}_{2} \mathrm{O}_{3}(10 \mathrm{~mL})$ and extracted with EtOAc $(2 \times 10 \mathrm{~mL})$. The combined organic layers were washed with brine $(10 \mathrm{~mL})$, dried over $\mathrm{Na}_{2} \mathrm{SO}_{4}$, filtered through cotton, and concentrated in vacuo. The resulting white solid was purified by flash column chromatography (10:90 to 40:60 EtOAc:hexanes) and then recrystallized using $\mathrm{Et}_{2} \mathrm{O}$ and $\mathrm{CH}_{2} \mathrm{Cl}_{2}$ to provide amide $\mathbf{S} 38$ as white needles $(0.03 \mathrm{~g}, 94 \%)$ :

$\mathrm{mp}=84-87^{\circ} \mathrm{C}$;

${ }^{1} \mathrm{H}$ NMR $\left(400 \mathrm{MHz}, \mathrm{CDCl}_{3}\right) \delta 7.29(\mathrm{~d}, J=8.1,2 \mathrm{H}), 7.19(\mathrm{~d}, J=8.1,2 \mathrm{H}), 6.56(\mathrm{br} \mathrm{s}, 1 \mathrm{H}), 5.51$ (br $\mathrm{s}, 1 \mathrm{H}), 5.02(\mathrm{~d}, J=4.1,1 \mathrm{H}), 4.85(\mathrm{~d}, J=4.1,1 \mathrm{H}), 3.86-3.96(\mathrm{~m}, 1 \mathrm{H}), 3.52-3.59(\mathrm{~m}, 1 \mathrm{H}), 2.36(\mathrm{~s}$, $3 \mathrm{H}), 1.22(\mathrm{t}, J=7.2,3 \mathrm{H}), 1.15(\mathrm{~s}, 9 \mathrm{H})$;

${ }^{13} \mathrm{C}$ NMR (100 MHz, $\left.\mathrm{CDCl}_{3}\right) 172.4(\mathrm{C}), 138.4(\mathrm{C}), 133.8(\mathrm{C}), 129.4(\mathrm{CH}), 126.6(\mathrm{CH}), 111.9(\mathrm{C})$, $108.1(\mathrm{CH}), 83.7(\mathrm{CH}), 65.2\left(\mathrm{CH}_{2}\right), 37.1(\mathrm{C}), 25.2\left(\mathrm{CH}_{3}\right), 21.4\left(\mathrm{CH}_{3}\right), 15.3\left(\mathrm{CH}_{3}\right)$;

IR (ATR) 3481, 2980, 1698, 1265, $1081 \mathrm{~cm}^{-1}$;

HRMS (ESI) m/z: [M + Na] ${ }^{+}$Calcd for $\mathrm{C}_{17} \mathrm{H}_{25} \mathrm{NaO}_{4}$ 330.1676; Found 330.1680.<smiles>CCOC(OCC)C(OC(=O)CC(C)C)c1cccc2ccccc12</smiles>

4c



$\mathrm{CH}_{2},-45^{\circ} \mathrm{C}$<smiles>CCO[C@@H]1O[C@](C#N)(C(C)(C)C)O[C@H]1c1cccc2ccccc12</smiles>

8c<smiles>CCO[C@@H]1O[C@](C)(C(C)(C)C)O[C@H]1c1cccc2ccccc12</smiles>

epi-8c

$\left(2 R^{\star}, 4 R^{\star}, 5 S^{\star}\right)-2-($ tert-Butyl)-4-ethoxy-5-(naphthalen-1-yl)-1,3-dioxolane-2-carbonitrile (8c) $\left(2 R^{\star}, 4 S^{\star}, 5 S^{\star}\right)$-2-(tert-butyl)-4-ethoxy-5-(naphthalen-1-yl)-1,3-dioxolane-2-carbonitrile (epi8c). Following the general procedure for acetal substitutions, acetal $4 \mathbf{c}(0.1 \mathrm{~g}, 0.3 \mathrm{mmol})$ in $\mathrm{CH}_{2} \mathrm{Cl}_{2}$ $(3 \mathrm{~mL})$ was mixed with trimethylsilyl cyanide $(0.145 \mathrm{~mL}, 1.16 \mathrm{mmol})$ and trimethylsilyl trifluoromethanesulfonate $(0.11 \mathrm{~mL}, 0.58 \mathrm{mmol})$ to give nitriles $8 \mathrm{c}$ and epi-8c as a $97: 3$ mixture of diastereomers. The mixture was purified by flash column chromatography (pentanes to 3:97 $\mathrm{Et}_{2} \mathrm{O}$ :hexanes) and then recrystallized from $\mathrm{Et}_{2} \mathrm{O}$ and $\mathrm{CH}_{2} \mathrm{Cl}_{2}$ to provide $\mathbf{8 c}$ as a yellow solid $(0.054$ g, 57\%):

$\mathrm{mp}=52-55^{\circ} \mathrm{C}$;

${ }^{1} \mathrm{H}$ NMR $\left(400 \mathrm{MHz}, \mathrm{CDCl}_{3}\right) \delta 8.18(\mathrm{~d}, J=9.7,1 \mathrm{H}), 7.91(\mathrm{dd}, J=7.1,2.2,1 \mathrm{H}), 7.86(\mathrm{~d}, J=8.2$, $1 \mathrm{H}), 7.68(\mathrm{~d}, J=7.1,1 \mathrm{H}), 7.54-7.57(\mathrm{~m}, 2 \mathrm{H}) 7.49(\mathrm{t}, J=7.6,1 \mathrm{H}), 5.94(\mathrm{~d}, J=3.4,1 \mathrm{H}), 5.18(\mathrm{~d}, J$ $=3.9,1 \mathrm{H}), 3.97(\mathrm{dq}, J=9.8,7.1,1 \mathrm{H}), 3.64(\mathrm{dq}, J=9.8,7.1,1 \mathrm{H}), 1.36(\mathrm{t}, J=7.2,3 \mathrm{H}), 1.27(\mathrm{~s}$, $9 \mathrm{H})$;

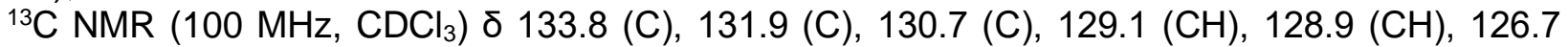
$(\mathrm{CH}), 126.2(\mathrm{CH}), 125.4(\mathrm{CH}), 123.6(\mathrm{CH}), 123.1(\mathrm{CH}), 117.3(\mathrm{C}), 107.6(\mathrm{CH}), 107.2(\mathrm{C}), 82.8$ $(\mathrm{CH}), 65.4\left(\mathrm{CH}_{2}\right), 38.0(\mathrm{C}), 24.4\left(\mathrm{CH}_{3}\right), 15.3\left(\mathrm{CH}_{3}\right)$;

IR (ATR) 2977, 2900, 2261, 1140, $1058 \mathrm{~cm}^{-1}$;

HRMS (APCl) m/z: [[M + H] $\left.-\mathrm{C}_{2} \mathrm{H}_{6} \mathrm{O}\right]^{+}$Calcd for $\mathrm{C}_{18} \mathrm{H}_{18} \mathrm{NO}_{2}$ 282.2489; Found 282.2489; 
HRMS (ESI) m/z: [M + Na] ${ }^{+}$Calcd for $\mathrm{C}_{20} \mathrm{H}_{23} \mathrm{NNaO}_{3}$ 348.1570; Found 348.1571.

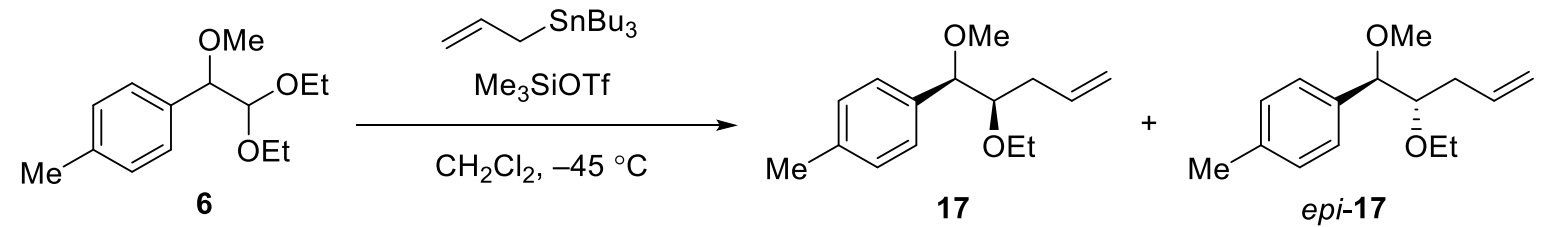


ethoxy-1-methoxypent-4-en-1-yl)-4-methylbenzene (epi-17). Following the general procedure for acetal substitutions, acetal $6(0.238 \mathrm{~g}, 1.00 \mathrm{mmol})$ in $\mathrm{CH}_{2} \mathrm{Cl}_{2}(10 \mathrm{~mL})$ was mixed with allyltributylstannane $(1.22 \mathrm{~mL}, 4.00 \mathrm{mmol})$ and trimethylsilyl trifluoromethanesulfonate $(0.364 \mathrm{~mL}$, $2.00 \mathrm{mmol}$ ) to provide 17 and epi-17 as a 61:39 mixture of diastereomers and as a colorless oil $(0.197 \mathrm{~g}, 85 \%)$. The diastereomers were separated by column chromatography, and the major diastereomer was characterized with an unidentifiable impurity. HRMS data for 17 and epi-17 could not be obtained because they are not stable.

Major diastereomer (17): ${ }^{1} \mathrm{H}$ NMR $\left(400 \mathrm{MHz}, \mathrm{CDCl}_{3}\right) \delta 7.22$ (d, $\left.J=7.9,2 \mathrm{H}\right), 7.16(\mathrm{~d}, J=7.9,2 \mathrm{H})$, 5.83- $5.90(\mathrm{~m}, 1 \mathrm{H}), 5.02-5.11(\mathrm{~m}, 2 \mathrm{H}), 4.06(\mathrm{~d}, J=5.7,1 \mathrm{H}), 3.34-3.38(\mathrm{~m}, 2 \mathrm{H}), 3.22(\mathrm{~s}, 3 \mathrm{H}), 2.35$ (s, 3H), 2.28-2.31 (br d, 3H), $0.99(\mathrm{t}, J=6.9,3 \mathrm{H})$;

${ }^{13} \mathrm{C}$ NMR (100 MHz, CDCl $\left.{ }_{3}\right) \delta 137.3(\mathrm{C}), 136.4(\mathrm{C}), 135.7(\mathrm{CH}), 128.9(\mathrm{CH}), 127.8(\mathrm{CH}), 116.8$ $\left(\mathrm{CH}_{2}\right), 85.3(\mathrm{CH}), 82.9(\mathrm{CH}), 66.5\left(\mathrm{CH}_{2}\right), 57.1\left(\mathrm{CH}_{3}\right), 35.9\left(\mathrm{CH}_{2}\right), 21.3\left(\mathrm{CH}_{3}\right), 15.5\left(\mathrm{CH}_{3}\right)$; IR (ATR) 2977, 1105, 915, 820, $736 \mathrm{~cm}^{-1}$.

Minor diastereomer (epi-17): ${ }^{1} \mathrm{H}$ NMR $\left(400 \mathrm{MHz}, \mathrm{CDCl}_{3}\right) \delta 7.20(\mathrm{~d}, J=7.9,2 \mathrm{H}), 7.16(\mathrm{~d}, J=7.9$, $2 \mathrm{H}), 5.78-5.88(\mathrm{~m}, 1 \mathrm{H}), 5.01-5.05(\mathrm{~m}, 2 \mathrm{H}), 4.15(\mathrm{~d}, J=5.2,1 \mathrm{H}), 3.48-3.56(\mathrm{~m}, 2 \mathrm{H}), 3.42-3.45$ $(\mathrm{m}, 1 \mathrm{H}), 3.26(\mathrm{~s}, 3 \mathrm{H}), 2.36(\mathrm{~s}, 3 \mathrm{H}), 2.18-2.21(\mathrm{~m}, 1 \mathrm{H}), 2.03-2.11(\mathrm{~m}, 1 \mathrm{H}), 1.14(\mathrm{t}, \mathrm{J}=6.9,3 \mathrm{H})$; ${ }^{13} \mathrm{C}$ NMR (100 MHz, $\left.\mathrm{CDCl}_{3}\right) \delta 137.3(\mathrm{C}), 136.3(\mathrm{C}), 135.4(\mathrm{CH}), 128.9(\mathrm{CH}), 127.6(\mathrm{CH}), 116.8$ $\left(\mathrm{CH}_{2}\right), 85.3(\mathrm{CH}), 82.7(\mathrm{CH}), 66.8\left(\mathrm{CH}_{2}\right), 57.2\left(\mathrm{CH}_{3}\right), 35.9\left(\mathrm{CH}_{2}\right), 21.3\left(\mathrm{CH}_{3}\right), 15.6\left(\mathrm{CH}_{3}\right)$.

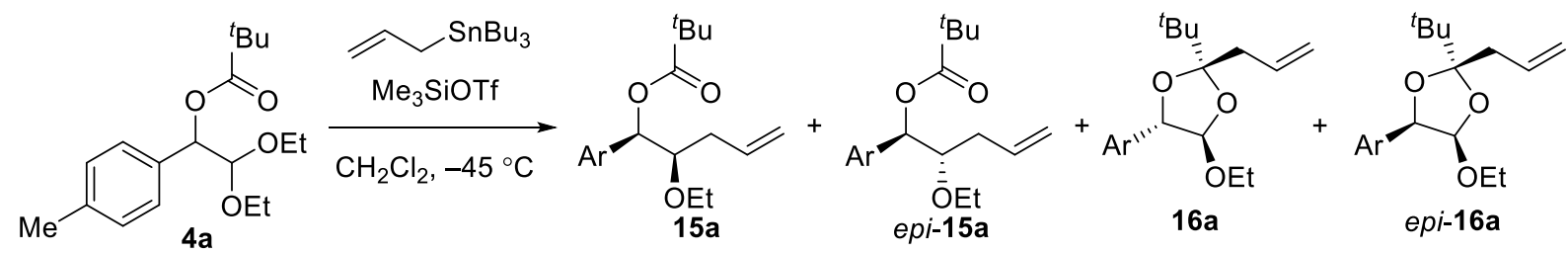

$\mathrm{Ar}=4-\mathrm{MeC}_{6} \mathrm{H}_{4}$

$\left(1 R^{\star}, 2 R^{\star}\right)$-2-Ethoxy-1-(p-tolyl)pent-4-en-1-yl pivaloate $\quad(15 a), \quad\left(1 R^{\star}, 2 S^{\star}\right)-2-$ ethoxy-1- $(p$ tolyl)pent-4-en-1-yl pivaloate (epi-15a), $\left(2 S^{\star}, 4 R^{\star}, 5 R^{\star}\right)-2$-allyl-2-(tert-butyl)-4-ethoxy-5-( $p$ tolyl)-1,3-dioxolane (16a), and $\left(2 S^{\star}, 4 R^{\star}, 5 S^{\star}\right)$-2-allyl-2-(tert-butyl)-4-ethoxy-5-( $p$-tolyl)-1,3dioxolane (epi-16a). Following the general procedure for acetal substitutions, acetal 4a (0.308 $\mathrm{g}, 1.00 \mathrm{mmol})$ in $\mathrm{CH}_{2} \mathrm{Cl}_{2}(10 \mathrm{~mL})$ was mixed with allyltributylstannane $(1.22 \mathrm{~mL}, 4.00 \mathrm{mmol})$ and trimethylsilyl trifluoromethanesulfonate $(0.364 \mathrm{~mL}, 2.00 \mathrm{mmol})$ to provide a 70:30 mixture ethers 15a and epi-15a to dioxolanes 16a and epi-16a. 15a and epi-15a were formed as an 88:12 mixture of diastereomers and as a colorless oil $(0.162 \mathrm{~g}, 53 \%)$, and the dioxolane 16a and epi-16a were formed as an 82:18 mixture of diastereomers and as a colorless oil ( $0.087 \mathrm{~g}, 28 \%) .21$ and epi15a were isolated as a 90:10 mixture of diastereomers and dioxolane 16a was isolated as a single diastereomer. 15a and epi-15a were characterized by ${ }^{1} \mathrm{H}$ and ${ }^{13} \mathrm{C}$ NMR using pure fractions that were obtained of each diastereomer, and IR, HRMS, and elemental analysis were obtained from the $90: 10$ mixture of diastereomers. 
Major diastereomer (15a): ${ }^{1} \mathrm{H}$ NMR $\left(400 \mathrm{MHz}, \mathrm{CDCl}_{3}\right) \delta 7.22(\mathrm{~d}, J=7.8,2 \mathrm{H}), 7.14(\mathrm{~d}, J=7.8$, 2H), 5.76-5.86 (ddt, $J=17.3,10.4,7.0,1 \mathrm{H}), 5.71(\mathrm{~d}, J=6.7,1 \mathrm{H}), 4.98-5.04(\mathrm{~m}, 2 \mathrm{H}), 3.53-3.63$ $(\mathrm{m}, 3 \mathrm{H}), 2.34(\mathrm{~s}, 3 \mathrm{H}), 2.10-2.17(\mathrm{~m}, 1 \mathrm{H}), 1.97-2.04(\mathrm{~m}, 1 \mathrm{H}), 1.23(\mathrm{~s}, 9 \mathrm{H}), 1.16(\mathrm{t}, \mathrm{J}=6.6,3 \mathrm{H})$;

${ }^{13} \mathrm{C}$ NMR (100 MHz, CDCl $\left.{ }_{3}\right)$ o $177.4(\mathrm{C}), 137.7(\mathrm{C}), 135.07(\mathrm{C}), 134.8(\mathrm{CH}), 129.1(\mathrm{CH}), 127.23$ $(\mathrm{CH}), 117.2\left(\mathrm{CH}_{2}\right), 81.3(\mathrm{CH}), 76.8(\mathrm{CH}), 66.7\left(\mathrm{CH}_{2}\right), 38.91(\mathrm{C}), 35.64\left(\mathrm{CH}_{2}\right), 27.28\left(\mathrm{CH}_{3}\right), 21.32$ $\left(\mathrm{CH}_{3}\right), 15.6\left(\mathrm{CH}_{3}\right)$;

IR (ATR) 2974, 1730, 1479, 1279, $813 \mathrm{~cm}^{-1}$;

HRMS (APCl) m/z: [[M + H] - $\left.\mathrm{C}_{5} \mathrm{H}_{10} \mathrm{O}_{2}\right]^{+}$Calcd for $\mathrm{C}_{14} \mathrm{H}_{19} \mathrm{O}$ 203.1430; Found 203.1427.

Minor diastereomer (epi-15a): ${ }^{1} \mathrm{H}$ NMR: $\left(400 \mathrm{MHz}, \mathrm{CDCl}_{3}\right) \delta 7.24(\mathrm{~d}, J=7.8,2 \mathrm{H}), 7.14(\mathrm{~d}, J=7.8$, $2 \mathrm{H}), 5.86(\mathrm{ddt}, J=17.6,10.8,6.9,1 \mathrm{H}), 5.74(\mathrm{~d}, J=5.5,1 \mathrm{H}), 5.04-5.09(\mathrm{~m}, 2 \mathrm{H}), 3.56(\mathrm{q}, J=5.5$, $1 \mathrm{H}), 3.45(\mathrm{q}, J=7.2,2 \mathrm{H}), 2.34(\mathrm{~s}, 3 \mathrm{H}), 2.26(\mathrm{t}, J=6.8,2 \mathrm{H}), 1.24(\mathrm{~s}, 9 \mathrm{H}), 1.08(\mathrm{t}, J=7.2,3 \mathrm{H})$;

${ }^{13} \mathrm{C}$ NMR $\left(100 \mathrm{MHz}, \mathrm{CDCl}_{3}\right) \delta 177.3(\mathrm{C}), 137.5(\mathrm{C}), 135.08$ (C, $\mathrm{CH}$ as determined by DEPT and HSQC), $128.9(\mathrm{CH}), 127.19(\mathrm{CH}), 117.1\left(\mathrm{CH}_{2}\right), 81.8(\mathrm{CH}), 75.9(\mathrm{CH}), 66.5\left(\mathrm{CH}_{2}\right), 38.94(\mathrm{C}), 35.63$ $\left(\mathrm{CH}_{2}\right), 27.29\left(\mathrm{CH}_{3}\right), 21.30\left(\mathrm{CH}_{3}\right), 15.52\left(\mathrm{CH}_{3}\right)$.

Dioxolane (16a): ${ }^{1} \mathrm{H}$ NMR $\left(400 \mathrm{MHz}, \mathrm{CDCl}_{3}\right) \delta 7.33(\mathrm{~d}, J=8.1,2 \mathrm{H}), 7.18(\mathrm{~d}, J=8.1,2 \mathrm{H}), 6.11$ (ddt, $J=17.1,10.1,6.8,1 \mathrm{H}), 5.15(\mathrm{~d}, J=17.1,1 \mathrm{H}), 5.09(\mathrm{~d}, J=10.3,1 \mathrm{H}), 4.96(\mathrm{~d}, J=5.7,1 \mathrm{H})$, $4.91(\mathrm{~d}, J=5.7,1 \mathrm{H}), 3.87(\mathrm{dq}, J=9.6,7.2,1 \mathrm{H}), 3.56(\mathrm{dq}, J=9.6,7.2,1 \mathrm{H}), 2.69-2.81(\mathrm{~m}, 2 \mathrm{H})$, $2.36(\mathrm{~s}, 3 \mathrm{H}), 1.25(\mathrm{t}, J=6.9,3 \mathrm{H}), 1.07(\mathrm{~s}, 9 \mathrm{H})$;

${ }^{13} \mathrm{C}$ NMR (100 MHz, $\left.\mathrm{CDCl}_{3}\right) 137.6(\mathrm{C}), 135.3(\mathrm{C}), 135.2(\mathrm{CH}), 129.2(\mathrm{CH}), 126.1(\mathrm{CH}), 116.7$ $\left(\mathrm{CH}_{2}\right), 115.9(\mathrm{C}), 108.2(\mathrm{CH}), 82.8(\mathrm{CH}), 65.1\left(\mathrm{CH}_{2}\right), 39.9(\mathrm{C}), 39.7\left(\mathrm{CH}_{2}\right), 25.9\left(\mathrm{CH}_{3}\right), 21.31\left(\mathrm{CH}_{3}\right)$, $15.53\left(\mathrm{CH}_{3}\right)$;

IR (ATR) 2976, 1516, 1392, 1082, $812 \mathrm{~cm}^{-1}$;

HRMS (APCl) m/z: [M + Na] $]^{+}$Calcd for $\mathrm{C}_{19} \mathrm{H}_{28} \mathrm{NaO}_{3}$ 327.1931; Found 327.1949.

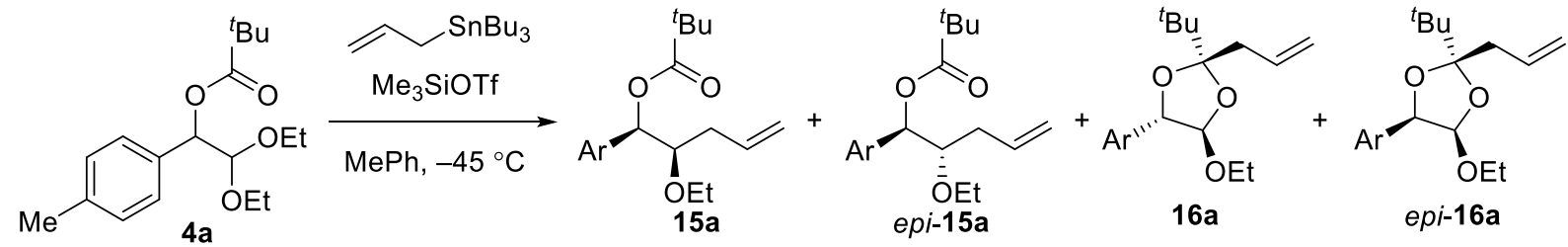
$\mathrm{Ar}=4-\mathrm{MeC}_{6} \mathrm{H}_{4}$

$\left(1 R^{\star}, 2 R^{\star}\right)$-2-Ethoxy-1-(p-tolyl)pent-4-en-1-yl pivaloate $\quad(15 \mathrm{a}), \quad\left(1 R^{\star}, 2 S^{\star}\right)-2-$ ethoxy-1- $(p$ tolyl)pent-4-en-1-yl pivaloate (epi-15a), $\left(2 S^{\star}, 4 R^{\star}, 5 R^{\star}\right)$-2-allyl-2-(tert-butyl)-4-ethoxy-5-( $p$ tolyl)-1,3-dioxolane (16a), and $\left(2 S^{\star}, 4 R^{\star}, 5 S^{\star}\right)$-2-allyl-2-(tert-butyl)-4-ethoxy-5-( $p$-tolyl)-1,3dioxolane (epi-16a). Following the general procedure for acetal substitutions, acetal 4 a $(0.308$ $\mathrm{mg}, 1.00 \mathrm{mmol})$ in toluene $(10 \mathrm{~mL})$ was mixed with allyltributylstannane $(1.22 \mathrm{~mL}, 4.00 \mathrm{mmol})$ and trimethylsilyl trifluoromethanesulfonate $(0.364 \mathrm{~mL}, 2.00 \mathrm{mmol})$. The resulting residue consisted of a 95:5 mixture of products, where 15a and epi-15a were formed as an 90:10 mixture of diastereomers as a colorless oil $(0.248 \mathrm{~g}, 82 \%)$, and dioxolane $16 \mathbf{a}$ and epi-16a were formed as a 64:46 mixture of diastereomers but was not isolated. 15a and epi-15a were isolated as a 90:10 mixture of diastereomers. The spectral data obtained are consistent with those reported above.<smiles>C=CC[C@@H](OC)C(C)(C)C</smiles> 
$\left(1 R^{\star}, 2 R^{\star}\right)$-2-Methoxy-1-(p-tolyl)pent-4-en-1-yl pivaloate $(20), \quad\left(1 R^{\star}, 2 S^{\star}\right)$-2-methoxy-1-( $p$ tolyl)pent-4-en-1-yl pivaloate (epi-20), $\left(2 S^{\star}, 4 R^{\star}, 5 R^{\star}\right)$-2,4-diallyl-2-(tert-butyl)-4-methoxy-5( $p$-tolyl)-1,3-dioxolane (22), and $\left(2 R^{\star}, 4 S^{\star}, 5 R^{\star}\right)$-2-allyl-2-(tert-butyl)-4-propyl-5-( $p$-tolyl)-1,3dioxolane (23). Following the general procedure for acetal substitutions, acetal $\mathbf{1 8}(0.28 \mathrm{~g}, 1.0$ $\mathrm{mmol})$ in $\mathrm{MePh}(10 \mathrm{~mL})$ was mixed with allyltributylstannane $(1.22 \mathrm{~mL}, 4.00 \mathrm{mmol})$ and trimethylsilyl trifluoromethanesulfonate $(0.364 \mathrm{~mL}, 2.00 \mathrm{mmol})$ to give a mixture of products $(\mathbf{2 0 : 2 2 : 2 3}=86: 7: 7) .20$ and epi-20 were formed as a mixture of diastereomers (20:epi-20 =76:24), the singly substituted dioxolane product $\mathbf{2 2}$ was formed as a mixture of diastereomers (22:epi-22 $=82: 18)$, and the disubstituted dioxolane product $\mathbf{2 3}$ was formed as a mixture of diastereomers (23:epi-23=53:47). 20 and epi-20 were isolated and characterized as an 83:17 mixture as a colorless oil $(0.226 \mathrm{~g}, 82 \%)$. Dioxolane 22 and epi-22 was isolated as a 93:7 mixture and as a colorless oil with tributylstannyl impurities $(0.016 \mathrm{~g}, 6 \%)$. Sufficient quantities of the minor diastereomer of the dioxolane product, epi-22, were not present to acquire a ${ }^{13} \mathrm{C}$ spectrum, and sufficiently pure material of $\mathbf{2 2}$ and epi-22 was not obtained to acquire HRMS data. The disubstituted dioxolane product 23 was obtained as a colorless oil $(0.018 \mathrm{~g}, 5 \%)$, but sufficient quantities of pure material were not obtained for complete characterization, and ${ }^{1} \mathrm{H}$ and ${ }^{13} \mathrm{C} N M R$ peak assignments were determined by comparing it to that of dioxolane 22.

20 and epi-20: ${ }^{1} \mathrm{H}$ NMR $\left(400 \mathrm{MHz}, \mathrm{CDCl}_{3}\right) \delta 7.22(\mathrm{~d}, J=7.9,2.4 \mathrm{H}), 7.13(\mathrm{~d}, J=7.9,2.4 \mathrm{H}), 5.77-$ $5.83(\mathrm{~m}, 1.2 \mathrm{H}), 5.74(\mathrm{~m}, 1.2 \mathrm{H}), 4.98-5.04(\mathrm{~m}, 2.4 \mathrm{H}), 3.44-3.49(\mathrm{~m}, 1.2 \mathrm{H}), 3.41(\mathrm{~s}, 3 \mathrm{H}), 3.32(\mathrm{~s}$, $0.6 \mathrm{H}), 2.31(\mathrm{~s}, 3.6 \mathrm{H}), 2.25(\mathrm{t}, J=6.7,0.4 \mathrm{H}), 1.98-2.18(\mathrm{~m}, 2 \mathrm{H}), 1.23(\mathrm{~s}, 2.5 \mathrm{H}), 1.22(\mathrm{~s}, 9 \mathrm{H})$;

Peaks in ${ }^{13} \mathrm{C} \mathrm{NMR} \mathrm{(100} \mathrm{MHz,} \mathrm{CDCl}_{3}$ ) attributed to the major diastereomer (20): 177.3 (C), 137.7 $(\mathrm{C}), 134.90(\mathrm{C}), 134.4(\mathrm{CH}), 129.0(\mathrm{CH}), 127.1(\mathrm{CH}), 117.3\left(\mathrm{CH}_{2}\right), 83.1(\mathrm{CH}), 76.3(\mathrm{CH}), 58.9$ $\left(\mathrm{CH}_{3}\right), 38.8(\mathrm{C}), 35.1\left(\mathrm{CH}_{2}\right), 27.18\left(\mathrm{CH}_{3}\right), 21.19\left(\mathrm{CH}_{3}\right)$;

Peaks in ${ }^{13} \mathrm{C}$ NMR (100 MHz, $\mathrm{CDCl}_{3}$ ) attributed to the minor diastereomer (epi-20): 177.1 (C), $137.5(\mathrm{C}), 134.86(\mathrm{C}), 134.7(\mathrm{CH}), 128.9(\mathrm{CH}), 127.0(\mathrm{CH}), 117.1\left(\mathrm{CH}_{2}\right), 83.5(\mathrm{CH}), 75.3(\mathrm{CH})$, $58.7\left(\mathrm{CH}_{3}\right), 38.9(\mathrm{C}), 34.9\left(\mathrm{CH}_{2}\right), 27.20\left(\mathrm{CH}_{3}\right), 21.20\left(\mathrm{CH}_{3}\right)$; IR (ATR) 2974, 1729, 1103, 838, $771 \mathrm{~cm}^{-1}$; HRMS (APCl) m/z: [[M + H] $\left.-\mathrm{C}_{5} \mathrm{H}_{10} \mathrm{O}_{2}\right]^{+}$Calcd for $\mathrm{C}_{13} \mathrm{H}_{17} \mathrm{O}$ 189.1274; Found 189.1273. Anal. Calcd for $\mathrm{C}_{18} \mathrm{H}_{26} \mathrm{O}_{3}: \mathrm{C}, 74.45 ; \mathrm{H}, 9.02$. Found: $\mathrm{C}, 74.03 ; \mathrm{H}, 9.01$.

Dioxolane (22): ${ }^{1} \mathrm{H}$ NMR $\left(400 \mathrm{MHz}, \mathrm{CDCl}_{3}\right) \delta 7.32(\mathrm{~d}, J=7.3,2 \mathrm{H}), 7.18(\mathrm{~d}, J=7.3,2 \mathrm{H}), 7.11(\mathrm{~d}$, $J=9.1,0.16 \mathrm{H}), 6.83(\mathrm{~d}, J=9.1,0.16 \mathrm{H}), 6.08(\mathrm{ddt}, J=17.2,10.1,7.3,1 \mathrm{H}), 5.81-5.89(\mathrm{~m}, 0.1 \mathrm{H})$, $5.14(\mathrm{~d}, J=17.9,1.08 \mathrm{H}), 5.14(\mathrm{~d}, J=10.2,1.08 \mathrm{H}), 4.97-5.02(\mathrm{~m}, 0.16 \mathrm{H}), 4.94(\mathrm{~d}, J=4.8,1 \mathrm{H})$, $4.81(\mathrm{~d}, J=4.8,1 \mathrm{H}), 3.80(\mathrm{~s}, 0.24 \mathrm{H}), 3.49(\mathrm{~s}, 3 \mathrm{H}), 2.64-2.80(\mathrm{~m}, 2.16 \mathrm{H}), 2.36(\mathrm{~s}, 3.3 \mathrm{H}), 1.09(\mathrm{~s}$, $0.5 \mathrm{H}), 1.07(\mathrm{~s}, 9 \mathrm{H})$;

${ }^{13} \mathrm{C}$ NMR (100 MHz, CDCl 3 ) 137.8 (C), $135.2(\mathrm{CH}), 135.1(\mathrm{C}), 129.3(\mathrm{CH}), 126.1(\mathrm{CH}), 116.8$ $\left(\mathrm{CH}_{2}\right), 116.2(\mathrm{C}), 109.4(\mathrm{CH}), 82.9(\mathrm{CH}), 56.8\left(\mathrm{CH}_{3}\right), 39.9(\mathrm{C}), 39.6\left(\mathrm{CH}_{2}\right), 25.9\left(\mathrm{CH}_{3}\right), 21.3\left(\mathrm{CH}_{3}\right)$; IR (ATR) 2966, 2922, 1054, 1032, $1015 \mathrm{~cm}^{-1}$.

Diallyl Dioxolane (23, characteristic peaks): ${ }^{1} \mathrm{H}$ NMR $\left(400 \mathrm{MHz}, \mathrm{CDCl}_{3}\right) \delta 7.20(\mathrm{~d}, J=8.1,2 \mathrm{H})$, $7.12(\mathrm{~d}, J=7.1,2 \mathrm{H}), 5.74-5.85(\mathrm{~m}, 2 \mathrm{H}), 5.71(\mathrm{~d}, J=6.2,1 \mathrm{H}), 5.00-5.02(\mathrm{~m}, 2 \mathrm{H}), 4.92-4.97(\mathrm{~m}$, 2H), 3.48-3.54 (m, 3H), $2.33(\mathrm{~s}, 3 \mathrm{H}), 1.22(\mathrm{~s}, 9 \mathrm{H})$;

${ }^{13} \mathrm{C} \mathrm{NMR}\left(100 \mathrm{MHz}, \mathrm{CDCl}_{3}\right) 138.9(\mathrm{C}), 137.5(\mathrm{C}), 134.6(\mathrm{CH}), 134.6(\mathrm{CH}), 128.9(\mathrm{CH}), 127.1(\mathrm{CH})$, 117.1 $\left(\mathrm{CH}_{2}\right), 114.3\left(\mathrm{CH}_{2}\right), 98.1(\mathrm{C}), 81.3(\mathrm{CH}), 76.5(\mathrm{CH}), 38.8(\mathrm{C}), 35.4\left(\mathrm{CH}_{2}\right), 33.7\left(\mathrm{CH}_{2}\right), 27.2$ $\left(\mathrm{CH}_{3}\right), 21.2\left(\mathrm{CH}_{3}\right)$. 

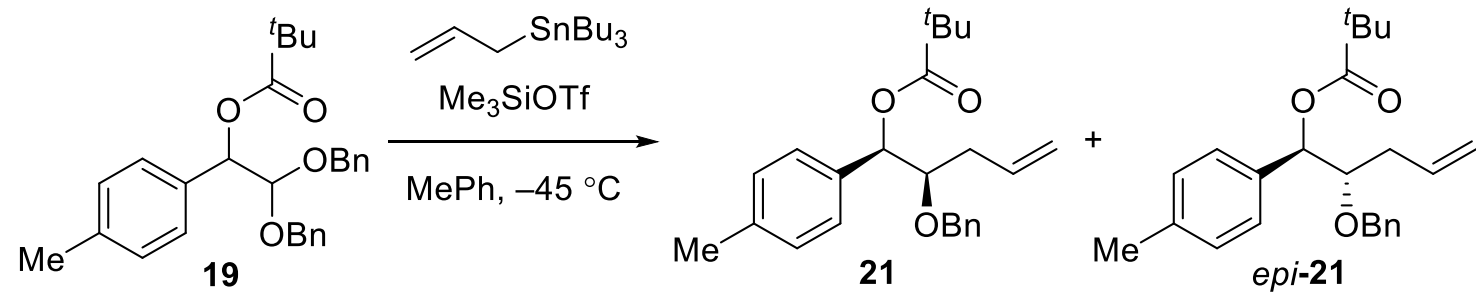

$\left(1 R^{\star}, 2 R^{\star}\right)$-2-(Benzyloxy)-1-(p-tolyl)pent-4-en-1-yl pivaloate

(21) and $\left(1 R^{\star}, 2 S^{\star}\right)-2-$ (benzyloxy)-1-(p-tolyl)pent-4-en-1-yl pivaloate (epi-21). Following the general procedure for acetal substitutions, acetal $19(0.263 \mathrm{~g}, 0.500 \mathrm{mmol})$ in $\mathrm{MePh}(5 \mathrm{~mL})$ was mixed with allyltributylstannane $(0.61 \mathrm{~mL}, 2.0 \mathrm{mmol})$ and trimethylsilyl trifluoromethanesulfonate $(0.182 \mathrm{~mL}$, $1.00 \mathrm{mmol})$ to give 21 and epi-21 as a mixture $(\mathbf{2 1}$ :epi-21 = 90:10) and as a colorless oil $(0.06 \mathrm{~g}$, 34\%). 21 and epi-21 were isolated and characterized as a 92:8 mixture with unidentifiable impurities:

${ }^{1} \mathrm{H}$ NMR $\left(400 \mathrm{MHz}, \mathrm{CDCl}_{3}\right)$ ठ 7.25-7.29 (m, 5H), 7.18-7.21 (m, 3H), 7.09-7.11 (m, 2.3H), 5.76$5.80(\mathrm{~m}, 2 \mathrm{H}), 5.76-5.80(\mathrm{~m}, 0.16 \mathrm{H}), 5.04-5.06(\mathrm{~m}, 0.16 \mathrm{H}), 4.95-5.01(\mathrm{~m}, 2 \mathrm{H}), 4.61(\mathrm{~d}, J=11.8$, $1 \mathrm{H}), 4.54(\mathrm{~d}, J=11.8,1 \mathrm{H}), 4.43(\mathrm{~d}, J=3.6,0.16 \mathrm{H}), 4.18-4.21(\mathrm{~m}, 0.1 \mathrm{H}), 3.71(\mathrm{q}, J=6.8,1 \mathrm{H})$, $2.3(\mathrm{~s}, 3.3 \mathrm{H}), 2.02-2.19(\mathrm{~m}, 2.16 \mathrm{H}), 1.21(\mathrm{~s}, 0.9 \mathrm{H}), 1.19(\mathrm{~s}, 9 \mathrm{H})$;

Peaks in ${ }^{13} \mathrm{C} \mathrm{NMR}\left(100 \mathrm{MHz}, \mathrm{CDCl}_{3}\right)$ attributed to the major diastereomer (21): $\delta 177.4(\mathrm{C}), 138.5$ $(\mathrm{C}), 137.8(\mathrm{C}), 135.0(\mathrm{C}), 134.5(\mathrm{CH}), 129.11(\mathrm{CH}), 128.4(\mathrm{CH}), 127.8(\mathrm{CH}), 127.6(\mathrm{CH}), 127.2$ $(\mathrm{CH}), 117.5\left(\mathrm{CH}_{2}\right), 81.1(\mathrm{CH}), 76.7(\mathrm{CH}), 72.9\left(\mathrm{CH}_{2}\right), 38.91(\mathrm{C}), 35.5\left(\mathrm{CH}_{2}\right), 27.3\left(\mathrm{CH}_{3}\right), 21.3$ $\left(\mathrm{CH}_{3}\right)$;

Peaks in ${ }^{13} \mathrm{C}$ NMR $\left(100 \mathrm{MHz}, \mathrm{CDCl}_{3}\right.$ ) attributed to the minor diastereomer (epi-21): $\delta 177.2$ (C), $138.3(\mathrm{C}), 137.6(\mathrm{C}), 134.79(\mathrm{C}), 134.75(\mathrm{CH}), 129.06(\mathrm{CH}), 128.9(\mathrm{CH}), 128.0(\mathrm{CH}), 127.5(\mathrm{CH})$, $127.3(\mathrm{CH}), 117.2\left(\mathrm{CH}_{2}\right), 81.3(\mathrm{CH}), 76.8(\mathrm{CH}), 72.7\left(\mathrm{CH}_{2}\right), 38.86(\mathrm{C}), 35.6\left(\mathrm{CH}_{2}\right), 29.1\left(\mathrm{CH}_{3}\right)$, $23.1\left(\mathrm{CH}_{3}\right)$;

IR (ATR) 2972, 2918, 1728, 1278, $1149 \mathrm{~cm}^{-1}$;

HRMS (ESI) m/z: [M + Na] ${ }^{+}$Calcd for $\mathrm{C}_{24} \mathrm{H}_{30} \mathrm{NaO}_{3}$ 389.2087; Found 389.2116.<smiles>CCOC(OCC)C(OC(=O)c1ccc(OC)cc1)c1ccc(C)cc1</smiles>

$7 b$

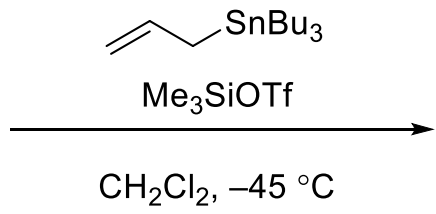

$\left(2^{\star} R, 4^{\star} \mathrm{S}, 5^{\star} \mathrm{R}\right)-2$-Allyl-4-ethoxy-2-(4-methoxyphenyl)-5-( $p$-tolyl)-1,3-dioxolane

Following the general procedure for acetal substitutions, acetal $7 \mathbf{b}(0.24 \mathrm{~g}, 0.66 \mathrm{mmol})$ in $\mathrm{CH}_{2} \mathrm{Cl}_{2}$ $(6.5 \mathrm{~mL})$ was combined with allyltributylstannane $(0.80 \mathrm{~mL}, 2.6 \mathrm{mmol})$ and trimethylsilyl trifluoromethanesulfonate $(0.239 \mathrm{~mL}, 1.31 \mathrm{mmol})$ to afford dioxolane $\mathbf{2 6} \mathbf{b}$ as a single diastereomer and as a colorless oil $(0.168 \mathrm{~g}, 72 \%)$ :

${ }^{1} \mathrm{H}$ NMR $\left(400 \mathrm{MHz}, \mathrm{CDCl}_{3}\right) \delta 7.41(\mathrm{~d}, J=8.5,2 \mathrm{H}), 7.10(\mathrm{~d}, J=8.2,2 \mathrm{H}), 7.01-7.03(\mathrm{~d}, J=8.2$, 2H), $6.82(\mathrm{~d}, J=8.5,2 \mathrm{H}), 5.61(\mathrm{ddt}, J=17.2,14.3,7.2,1 \mathrm{H}), 5.03(\mathrm{~d}, J=3.3,1 \mathrm{H}), 4.96(\mathrm{dd}, J=$ 10.1, 3.7, 3H), $3.85(\mathrm{dq}, J=9.5,7.1,1 \mathrm{H}), 3.75(\mathrm{~s}, 3 \mathrm{H}), 3.49(\mathrm{dq}, J=9.5,7.1,1 \mathrm{H}), 2.87-2.99(\mathrm{~m}$, $2 \mathrm{H}), 2.24(\mathrm{~s}, 3 \mathrm{H}), 1.20(\mathrm{t}, J=7.1,3 \mathrm{H})$; 
${ }^{13} \mathrm{C} \mathrm{NMR}\left(100 \mathrm{MHz}, \mathrm{CDCl}_{3}\right) \delta 159.4(\mathrm{C}), 137.9(\mathrm{C}), 135.6(\mathrm{C}), 134.4(\mathrm{C}), 133.2(\mathrm{CH}), 129.3(\mathrm{CH})$, $126.9(\mathrm{CH}), 126.6(\mathrm{CH}), 118.2(\mathrm{C}), 113.4\left(\mathrm{CH}_{2}\right), 112.1(\mathrm{CH}), 108.1(\mathrm{CH}), 84.3(\mathrm{CH}), 64.7\left(\mathrm{CH}_{2}\right)$, $55.4\left(\mathrm{CH}_{3}\right), 45.4\left(\mathrm{CH}_{2}\right), 21.3\left(\mathrm{CH}_{3}\right), 15.4\left(\mathrm{CH}_{3}\right)$;

IR (ATR) 2956, 2921, 1247, 1032, $973 \mathrm{~cm}^{-1}$;

HRMS (ESI) m/z: [M + Na] ${ }^{+}$Calcd for $\mathrm{C}_{22} \mathrm{H}_{26} \mathrm{NaO}_{4}$ 377.1723; Found 377.1735.

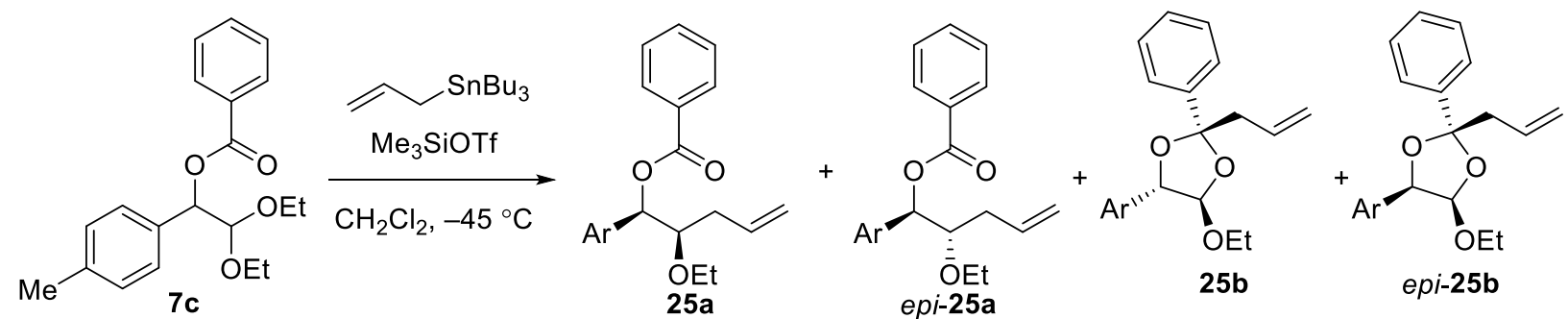

$\left(1 R^{\star}, 2 R^{\star}\right)$-2-Ethoxy-1-(p-tolyl)pent-4-en-1-yl benzoate $\quad(25 a), \quad\left(1 R^{\star}, 2 S^{\star}\right)-2$-ethoxy-1- $(p$ tolyl)pent-4-en-1-yl benzoate (epi-25a), $\left(2 S^{\star}, 4 R^{\star}, 5 R^{\star}\right)$-2-allyl-4-ethoxy-2-phenyl-5-(p-tolyl)1,3-dioxolane (25b), and $\left(2 S^{\star}, 4 R^{\star}, 5 S^{\star}\right)$-2-allyl-4-ethoxy-2-phenyl-5-(p-tolyl)-1,3-dioxolane (epi-25b). Following the general procedure for acetal substitutions, a solution of acetal 7c (0.36 $\mathrm{g}, 1.0 \mathrm{mmol})$ in $\mathrm{CH}_{2} \mathrm{Cl}_{2}(10 \mathrm{~mL})$ was mixed with allyltributylstannane $(1.22 \mathrm{~mL}, 4.00 \mathrm{mmol})$ and trimethylsilyl trifluoromethanesulfonate $(0.364 \mathrm{~mL}, 2.00 \mathrm{mmol})$ to provide $25 \mathrm{a}$ and epi-25a and 25b and epi-25b as a 79:21 mixture and as a colorless oil (0.194 g, 54\%). 25a and epi-25a were formed as an 85:15 mixture of diastereomers and dioxolane $\mathbf{2 5 b}$ and epi-25b were formed as a 67:33 mixture of diastereomers. Sufficient quantities of dioxolane $\mathbf{2 5 b}$ and epi-25b were not obtained for characterization. 25a and epi-25a were characterized as an 89:11 mixture of diastereomers:

${ }^{1} \mathrm{H}$ NMR $\left(400 \mathrm{MHz}, \mathrm{CDCl}_{3}\right) \delta 8.11(\mathrm{~d}, J=7.5,2.1 \mathrm{H}), 7.57(\mathrm{t}, J=7.4,1.12 \mathrm{H}), 7.47(\mathrm{t}, J=7.9$, $2.12 \mathrm{H}), 7.35(\mathrm{~d}, J=7.9,2.12 \mathrm{H}), 7.16(\mathrm{~d}, J=7.9,2.12 \mathrm{H}), 6.04(\mathrm{~d}, J=4.7,1 \mathrm{H}), 6.01(\mathrm{br} \mathrm{s}, 0.12 \mathrm{H})$, 5.86-5.93 (m, 1H), 5.75-5.77 (m, 0.12H), 5.09 (dd overlapped, $J=9.5,2 \mathrm{H}), 4.99(\mathrm{~d}, J=8.4$, $0.12 \mathrm{H}), 4.92(\mathrm{~d}, J=10.5,0.12 \mathrm{H}), 3.73(\mathrm{q}, J=5.3,1.12 \mathrm{H}), 3.51-3.59(\mathrm{~m}, 2.2 \mathrm{H}), 2.34(\mathrm{br} \mathrm{s}, 3.4 \mathrm{H})$, $1.13(\mathrm{t}, J=6.8,3 \mathrm{H}), 0.89(\mathrm{t}, J=6.8,0.4 \mathrm{H})$;

Peaks in ${ }^{13} \mathrm{C} \mathrm{NMR}\left(100 \mathrm{MHz}, \mathrm{CDCl}_{3}\right)$ attributed to the major diastereomer (25a): $\delta 165.6(\mathrm{C})$, $137.8(\mathrm{C}), 134.98(\mathrm{CH}), 134.76(\mathrm{C}), 133.1(\mathrm{C}), 130.5(\mathrm{CH}), 129.9(\mathrm{CH}), 129.1(\mathrm{CH}), 128.54$ $(\mathrm{CH}), 127.4(\mathrm{CH}), 117.4\left(\mathrm{CH}_{2}\right), 81.8(\mathrm{CH}), 76.9(\mathrm{CH}), 66.7\left(\mathrm{CH}_{2}\right), 35.7\left(\mathrm{CH}_{2}\right), 21.3\left(\mathrm{CH}_{3}\right), 15.6$ $\left(\mathrm{CH}_{3}\right)$;

Peaks in ${ }^{13} \mathrm{C}$ NMR $\left(100 \mathrm{MHz}, \mathrm{CDCl}_{3}\right)$ attributed to the minor diastereomer (25a): $\delta 165.7(\mathrm{C})$, $138.0(\mathrm{C}), 134.89(\mathrm{CH}), 134.6(\mathrm{C}), 133.0(\mathrm{C}), 130.6(\mathrm{CH}), 129.8(\mathrm{CH}), 129.2(\mathrm{CH}), 128.51(\mathrm{CH})$, $127.3(\mathrm{CH}), 117.2\left(\mathrm{CH}_{2}\right), 81.7(\mathrm{CH}), 77.4(\mathrm{CH}), 71.6\left(\mathrm{CH}_{2}\right), 33.8\left(\mathrm{CH}_{2}\right), 22.8\left(\mathrm{CH}_{3}\right), 14.3\left(\mathrm{CH}_{3}\right)$; IR (ATR) 2974, 1718, 1265, 1095, $1068 \mathrm{~cm}^{-1}$;

HRMS (APCl) m/z: [[M + H] - $\left.\mathrm{C}_{7} \mathrm{H}_{6} \mathrm{O}_{2}\right]^{+}$Calcd for $\mathrm{C}_{14} \mathrm{H}_{19} \mathrm{O}$ 203.1430; Found 203.1429.

<smiles>C=CC[C@H](OCC)[C@H](OC(=O)c1ccc([N+](=O)[O-])cc1)c1ccc(C)cc1</smiles><smiles>C=CC[C@H](OCC)[C@H](OC(=O)c1ccc([N+](=O)[O-])cc1)c1ccc(C)cc1</smiles> 
$\left(1 R^{\star}, 2 R^{\star}\right)$-2-Ethoxy-1-( $p$-tolyl)pent-4-en-1-yl 4-nitrobenzoate $(24 a)$ and $\left(1 R^{\star}, 2 S^{\star}\right)$-2-ethoxy1-(p-tolyl)pent-4-en-1-yl 4-nitrobenzoate (epi-24a). Following the general procedure for acetal substitutions, a solution of acetal $7 \mathrm{a}(0.092 \mathrm{~g}, 0.25 \mathrm{mmol})$ in $\mathrm{CH}_{2} \mathrm{Cl}_{2}(2.5 \mathrm{~mL})$ was mixed with allyltributylstannane $(0.31 \mathrm{~mL}, 1.0 \mathrm{mmol})$ and trimethylsilyl trifluoromethanesulfonate $(0.091 \mathrm{~mL}$, $0.50 \mathrm{mmol}$ ) to give 24a and epi-24a as a 78:22 mixture of diastereomers. The mixture was purified by flash column chromatography (5:95 to $20: 80 \mathrm{Et}_{2} \mathrm{O}$ :hexanes) to provide $24 \mathrm{a}$ and epi-24a as a 79:21 mixture of diastereomers and as a colorless oil $(0.04 \mathrm{~g}, 43 \%)$ :

${ }^{1} \mathrm{H}$ NMR $\left(400 \mathrm{MHz}, \mathrm{CDCl}_{3}\right) \delta 8.31(\mathrm{~m}, J=8.7,2.5 \mathrm{H}), 8.27(\mathrm{~m}, 2.5 \mathrm{H}), 7.34(\mathrm{~d}, J=7.9,2.5 \mathrm{H}), 7.18$ $(\mathrm{d}, J=7.9,2.5 \mathrm{H}), 6.07(\mathrm{~d}, J=6.6,0.25 \mathrm{H}), 6.01(\mathrm{~d}, J=6.6,1 \mathrm{H}), 5.80-5.91(\mathrm{~m}, 1.25 \mathrm{H}), 5.01-5.10$ $(\mathrm{m}, 2.5 \mathrm{H}), 3.73-3.77(\mathrm{~m}, 1.25 \mathrm{H}), 3.52-3.68(\mathrm{~m}, 2.5 \mathrm{H}), 2.5(\mathrm{~s}, 3.75 \mathrm{H}), 2.09-2.32(\mathrm{~m}, 2.5 \mathrm{H}), 1.13$ (t, $J=7.1,3.8 \mathrm{H})$;

Peaks in $\left.{ }^{13} \mathrm{C} \mathrm{NMR} \mathrm{(100} \mathrm{MHz,} \mathrm{CDCl}_{3}\right)$ attributed to the major diastereomer (24a): $\delta 163.9(\mathrm{C})$, $150.72(\mathrm{C}), 138.5(\mathrm{C}), 136.0(\mathrm{C}), 134.3(\mathrm{C}), 134.1(\mathrm{CH}), 130.87(\mathrm{CH}), 129.4(\mathrm{CH}), 127.5(\mathrm{CH})$, $123.70(\mathrm{CH}), 117.5\left(\mathrm{CH}_{2}\right), 81.4(\mathrm{CH}), 78.9(\mathrm{CH}), 67.1\left(\mathrm{CH}_{2}\right), 35.5\left(\mathrm{CH}_{2}\right), 27.9\left(\mathrm{CH}_{3}\right), 15.7\left(\mathrm{CH}_{3}\right)$; Peaks in ${ }^{13} \mathrm{C}$ NMR $\left(100 \mathrm{MHz}, \mathrm{CDCl}_{3}\right)$ attributed to the minor diastereomer (epi-24a): $\delta 163.8$ $(\mathrm{C}), 150.66(\mathrm{C}), 138.2(\mathrm{C}), 135.9(\mathrm{C}), 134.7(\mathrm{C}), 134.0(\mathrm{CH}), 130.92(\mathrm{CH}), 129.2(\mathrm{CH}), 127.4$ $(\mathrm{CH}), 123.75(\mathrm{CH}), 117.8\left(\mathrm{CH}_{2}\right), 81.6(\mathrm{CH}), 77.9(\mathrm{CH}), 66.7\left(\mathrm{CH}_{2}\right), 35.8\left(\mathrm{CH}_{2}\right), 27.8\left(\mathrm{CH}_{3}\right), 15.6$ $\left(\mathrm{CH}_{3}\right)$;

IR (ATR) 2956, 2921, 1247, 1032, $973 \mathrm{~cm}^{-1}$;

HRMS (APCl) m/z: [[M + H] $\left.-\mathrm{C}_{7} \mathrm{H}_{5} \mathrm{NO}_{4}\right]^{+}$Calcd for $\mathrm{C}_{14} \mathrm{H}_{19} \mathrm{O}$ 203.1430; Found 203.1428.<smiles>CCCNC(=O)OC(c1ccc(C)cc1)C(OCC)OCC</smiles>

7d

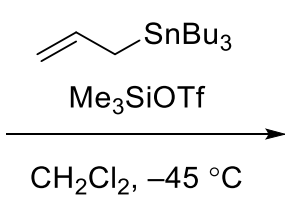

<smiles>C=CC[C@H](OCC)[C@H](OC(=O)NCCC)c1ccc(C)cc1</smiles>

$27 a$<smiles>C=CC[C@H](OCC)[C@H](OC(=O)NCCC)c1ccc(C)cc1</smiles>

epi-27a

$\left(1 R^{\star}, 2 R^{\star}\right)$-2-Ethoxy-1-(p-tolyl)pent-4-en-1-yl tert-butylcarbamate (27a) and $\left(1 R^{\star}, 2 S^{\star}\right)$-2ethoxy-1-(p-tolyl)pent-4-en-1-yl tert-butylcarbamate (epi-27a). Following the general procedure for acetal substitutions, acetal $7 \mathbf{d}(0.050 \mathrm{~g}, 0.15 \mathrm{mmol})$ in $\mathrm{CH}_{2} \mathrm{Cl}_{2}(1.5 \mathrm{~mL})$ was mixed with allyltributylstannane $(0.19 \mathrm{~mL}, 0.62 \mathrm{mmol})$ and trimethylsilyl trifluoromethanesulfonate $(0.056$ $\mathrm{mL}, 0.31 \mathrm{mmol}$ ) to give $27 \mathrm{a}$ and epi-27a as a 78:22 mixture of diastereomers. The mixture was purified by flash column chromatography (pentanes to 3:97 to 10:90 $\mathrm{Et}_{2} \mathrm{O}$ :hexanes) to provide 27a and epi-27a as a colorless oil $(0.042 \mathrm{~g}, 88 \%)$. 27a and epi-27a were characterized by ${ }^{1} \mathrm{H}$ and ${ }^{13} \mathrm{C}$ NMR using pure fractions that were obtained of each diastereomer, and IR and HRMS were obtained from a 78:22 mixture of diastereomers.

Major diastereomer (27a): ${ }^{1} \mathrm{H}$ NMR $\left(400 \mathrm{MHz}, \mathrm{CDCl}_{3}\right) \delta 7.23(\mathrm{~d}, J=7.6,2 \mathrm{H}), 7.14(\mathrm{~d}, J=7.6$, $2 \mathrm{H}), 5.76-5.86(\mathrm{~m}, 1 \mathrm{H}), 5.72(\mathrm{~d}, J=6.3,1 \mathrm{H}), 4.98-5.04(\mathrm{~m}, 2 \mathrm{H}), 3.56-3.61(\mathrm{~m}, 3 \mathrm{H}), 2.34(\mathrm{~s}, 3 \mathrm{H})$, 2.12-2.16 (m, 1H), 1.97-2.05 (m, 1H), $1.23(\mathrm{~s}, 9 \mathrm{H}), 1.16(\mathrm{t}, J=6.9,3 \mathrm{H})$;

${ }^{13} \mathrm{C}$ NMR (100 MHz, CDCl $\left.{ }_{3}\right) \delta 177.4(\mathrm{C}), 137.7(\mathrm{C}), 135.0(\mathrm{C}), 134.7(\mathrm{CH}), 129.1(\mathrm{CH}), 127.20$ $(\mathrm{CH}), 117.2\left(\mathrm{CH}_{2}\right), 81.3(\mathrm{CH}), 76.7(\mathrm{CH}), 66.7\left(\mathrm{CH}_{2}\right), 38.88(\mathrm{C}), 35.61\left(\mathrm{CH}_{2}\right), 27.2\left(\mathrm{CH}_{3}\right), 21.28$ $\left(\mathrm{CH}_{3}\right), 15.6\left(\mathrm{CH}_{3}\right)$;

IR (ATR) 2974, 1730, 1479, 1279, $813 \mathrm{~cm}^{-1}$;

HRMS (APCl) m/z: [[M + H] - $\left.\mathrm{C}_{5} \mathrm{H}_{11} \mathrm{NO}_{2}\right]^{+}$Calcd for $\mathrm{C}_{14} \mathrm{H}_{19} \mathrm{O}$ 203.1430; Found 203.1427.

Minor diastereomer (epi-27a): ${ }^{1} \mathrm{H}$ NMR: $\left(400 \mathrm{MHz}, \mathrm{CDCl}_{3}\right) \delta 7.24(\mathrm{~d}, J=7.8,2 \mathrm{H}), 7.14(\mathrm{~d}, J=7.8$, $2 \mathrm{H}), 5.81-5.91(\mathrm{~m}, 1 \mathrm{H}), 5.75(\mathrm{~d}, J=5.5,1 \mathrm{H}), 5.04-5.09(\mathrm{~m}, 2 \mathrm{H}), 3.56(\mathrm{q}, J=5.5,1 \mathrm{H}), 3.47(\mathrm{q}, J$ $=7.2,2 \mathrm{H}), 2.34(\mathrm{~s}, 3 \mathrm{H}), 2.26(\mathrm{t}, J=6.8,1 \mathrm{H}), 1.63-1.69(\mathrm{~m}, 1 \mathrm{H}), 1.24(\mathrm{~s}, 9 \mathrm{H}), 1.09(\mathrm{t}, J=7.2,3 \mathrm{H})$; 
${ }^{13} \mathrm{C}$ NMR $\left(100 \mathrm{MHz}, \mathrm{CDCl}_{3}\right) \delta 177.3(\mathrm{C}), 137.5(\mathrm{C}), 135.1(\mathrm{CH}, \mathrm{C}$ as determined by DEPT and HSQC), $128.9(\mathrm{CH}), 127.21(\mathrm{CH}), 117.1\left(\mathrm{CH}_{2}\right), 81.8(\mathrm{CH}), 75.9(\mathrm{CH}), 66.6\left(\mathrm{CH}_{2}\right), 38.96(\mathrm{C}), 35.65$ $\left(\mathrm{CH}_{2}\right), 27.9\left(\mathrm{CH}_{3}\right), 21.32\left(\mathrm{CH}_{3}\right), 15.5\left(\mathrm{CH}_{3}\right)$.<smiles>C=CC[C@H](OCC)[C@H](OC(=O)C(C)(C)C)c1ccc(C)cc1</smiles>

$\left(1 R^{\star}, 2 R^{\star}\right)$-2-Ethoxy-1-(p-tolyl)pent-4-en-1-yl pivaloate (15a) and $\left(1 R^{\star}, 2 S^{\star}\right)$-2-ethoxy-1-(ptolyl)pent-4-en-1-yl pivaloate (epi-15a). Following the general procedure for acetal substitutions, acetal $4 a(0.154 \mathrm{~g}, 0.500 \mathrm{mmol})$ in $\mathrm{CH}_{2} \mathrm{Cl}_{2}(5 \mathrm{~mL})$ was mixed with allyltrimethylsilane $(0.32 \mathrm{~mL}, 2.0 \mathrm{mmol})$ and trimethylsilyl trifluoromethanesulfonate $(0.182 \mathrm{~mL}, 1.00 \mathrm{mmol})$ to afford 15a and epi-15a as a 54:46 mixture of diastereomers and as a colorless oil $(0.132 \mathrm{~g}, 87 \%)$. The spectral data obtained are consistent with those reported above.



$\left(1 R^{\star}, 2 R^{\star}\right)$-2-ethoxy-4-methyl-1-( $p$-tolyl)pent-4-en-1-yl pivaloate (29) and $\left(1 R^{\star}, 2 S^{\star}\right)$-2-ethoxy4-methyl-1-(p-tolyl)pent-4-en-1-yl pivaloate (epi-29). Following the general procedure for acetal substitutions, acetal $4 \mathrm{a}(0.154 \mathrm{~g}, 0.500 \mathrm{mmol})$ in $\mathrm{CH}_{2} \mathrm{Cl}_{2}(5 \mathrm{~mL})$ was mixed with methallyltrimethylsilane $(0.35 \mathrm{~mL}, 2.0 \mathrm{mmol})$ and trimethylsilyl trifluoromethanesulfonate $(0.182$ $\mathrm{mL}, 1.00 \mathrm{mmol}$ ) to afford 29 and epi-29 as a 70:30 mixture of diastereomers and as a colorless oil $(0.142 \mathrm{~g}, 89 \%)$. The spectral data obtained are consistent with those reported below.



$$
\mathrm{Ar}=4-\mathrm{MeC}_{6} \mathrm{H}_{4}
$$

$\left(1 R^{\star}, 2 R^{\star}\right)$-2-Ethoxy-4-methyl-1-(p-tolyl)pent-4-en-1-yl pivaloate (29), $\left(1 R^{\star}, 2 S^{\star}\right)$-2-ethoxy-4methyl-1-( $p$-tolyl)pent-4-en-1-yl pivaloate (epi-29), and $\left(2 S^{\star}, 4 R^{\star}, 5 R^{\star}\right)-2$-(tert-butyl)-4ethoxy-2-(2-methylallyl)-5-(p-tolyl)-1,3-dioxolane (S39). Following the general procedure for acetal substitutions, acetal $4 \mathrm{a}(0.20 \mathrm{~g}, 0.65 \mathrm{mmol})$ in $\mathrm{MePh}(6.5 \mathrm{~mL})$ was mixed with methallyltributylstannane $(0.897 \mathrm{~g}, 2.60 \mathrm{mmol})$ and trimethylsilyl trifluoromethanesulfonate $(0.238$ $\mathrm{mL}, 1.30 \mathrm{mmol}$ ) to afford ether 29 and epi-29 and dioxolane S39 as a 95:5 mixture. 29 and epi29 were formed as a 93:7 mixture of diastereomers and as a colorless oil (0.093 g, 45\%), and dioxolane S39 was formed as a single diastereomer and as a colorless oil $(0.01 \mathrm{~g}, 3 \%)$. 29 and epi-29 were isolated as a 90:10 mixture of diastereomers and the dioxolane product, S39, was 
isolated as a single diastereomer. 29 and epi-29 were characterized by ${ }^{1} \mathrm{H}$ and ${ }^{13} \mathrm{C}$ NMR using pure fractions that were obtained of each diastereomer, and IR and HRMS were obtained from a 90:10 mixture of diastereomers. The minor diastereomer, epi-29, was characterized with tributylstannyl impurities.

Major diastereomer (29): ${ }^{1} \mathrm{H}$ NMR (400 MHz, $\left.\mathrm{CDCl}_{3}\right) \delta 7.22$ (d, $\left.J=7.7,2 \mathrm{H}\right), 7.12(\mathrm{~d}, J=7.7,2 \mathrm{H})$, $5.72(\mathrm{~d}, J=5.6,1 \mathrm{H}), 4.76(\mathrm{~s}, 1 \mathrm{H}), 4.69(\mathrm{~s}, 1 \mathrm{H}), 3.61-3.67(\mathrm{~m}, 2 \mathrm{H}), 3.51-3.59(\mathrm{~m}, 1 \mathrm{H}), 3.32(\mathrm{~s}$, $3 \mathrm{H}), 1.92-2.06(\mathrm{~m}, 2 \mathrm{H}), 1.71(\mathrm{~s}, 3 \mathrm{H}), 1.23(\mathrm{~s}, 9 \mathrm{H}), 1.13(\mathrm{t}, J=7.4,3 \mathrm{H})$;

${ }^{13} \mathrm{C}$ NMR (100 MHz, CDCl 3 ) ס $177.4(\mathrm{C}), 142.5(\mathrm{C}), 137.57(\mathrm{C}), 135.1(\mathrm{C}), 128.9(\mathrm{CH}), 127.12$ $(\mathrm{CH}), 112.9\left(\mathrm{CH}_{2}\right), 80.3(\mathrm{CH}), 76.5(\mathrm{CH}), 66.7\left(\mathrm{CH}_{2}\right), 39.5\left(\mathrm{CH}_{2}\right), 38.9\left(\mathrm{CH}_{3}\right), 27.3(\mathrm{C}), 22.96$ $\left(\mathrm{CH}_{3}\right), 21.27\left(\mathrm{CH}_{3}\right), 15.6\left(\mathrm{CH}_{3}\right)$;

IR (ATR) 2973, 1730, 1149, 812, $769 \mathrm{~cm}^{-1}$;

HRMS (APCl) m/z: [[M + H] $\left.-\mathrm{C}_{5} \mathrm{H}_{10} \mathrm{O}_{2}\right]^{+}$Calcd for $\mathrm{C}_{15} \mathrm{H}_{21} \mathrm{O} 217.1587$; Found 217.1585.

Minor diastereomer (epi-29): ${ }^{1} \mathrm{H}$ NMR $\left(400 \mathrm{MHz}, \mathrm{CDCl}_{3}\right) \delta 7.24(\mathrm{~d}, J=7.9,2 \mathrm{H}), 7.14(\mathrm{~d}, J=7.9$, $2 \mathrm{H}), 5.77(\mathrm{~d}, J=4.7,1 \mathrm{H}), 4.79(\mathrm{~s}, 1 \mathrm{H}), 4.73(\mathrm{~s}, 1 \mathrm{H}), 3.66-3.70(\mathrm{~m}, 1 \mathrm{H}), 3.41-3.54(\mathrm{~m}, 2 \mathrm{H}), 2.34$ $(\mathrm{s}, 3 \mathrm{H}), 2.16(\mathrm{~d}, J=6.1,2 \mathrm{H}), 1.75(\mathrm{~s}, 3 \mathrm{H}), 1.25(\mathrm{~s}, 9 \mathrm{H}), 1.09(\mathrm{t}, J=7.5,3 \mathrm{H})$;

${ }^{13} \mathrm{C} \mathrm{NMR}\left(100 \mathrm{MHz}, \mathrm{CDCl}_{3}\right) \delta 177.3(\mathrm{C}), 142.6(\mathrm{C}), 137.4(\mathrm{C}), 134.8(\mathrm{C}), 128.8(\mathrm{CH}), 127.13(\mathrm{CH})$, $112.5\left(\mathrm{CH}_{2}\right), 80.6(\mathrm{CH}), 76.1(\mathrm{CH}), 66.4\left(\mathrm{CH}_{2}\right), 39.3\left(\mathrm{CH}_{2}\right), 38.8\left(\mathrm{CH}_{3}\right), 27.2(\mathrm{C}), 22.94\left(\mathrm{CH}_{3}\right), 21.2$ $\left(\mathrm{CH}_{3}\right), 15.4\left(\mathrm{CH}_{3}\right)$.

Dioxolane (S39): ${ }^{1} \mathrm{H}$ NMR $\left(400 \mathrm{MHz}, \mathrm{CDCl}_{3}\right) \delta 7.33(\mathrm{~d}, J=7.9,2 \mathrm{H}), 7.18(\mathrm{~d}, J=7.9,2 \mathrm{H}), 4.95(\mathrm{~d}$, $J=5.7,1 \mathrm{H}), 4.89(\mathrm{~d}, J=5.3,2 \mathrm{H}), 4.86(\mathrm{~d}, J=5.6,1 \mathrm{H}), 3.85(\mathrm{dq}, J=9.7,7.2,1 \mathrm{H}), 3.56(\mathrm{dq}, J=$ 9.7, 7.2, 1H), 2.66 (br s, 2H), $2.36(\mathrm{~s}, 3 \mathrm{H}), 1.93(\mathrm{~s}, 3 \mathrm{H}), 1.24(\mathrm{t}, J=7.3,3 \mathrm{H}), 1.07(\mathrm{~s}, 9 \mathrm{H})$;

${ }^{13} \mathrm{C}$ NMR (100 MHz, CDCl 3 ) $\delta 143.2(\mathrm{C}), 137.58(\mathrm{C}), 135.3(\mathrm{C}), 129.2(\mathrm{CH}), 125.9(\mathrm{CH}), 116.5$ $(\mathrm{C}), 115.3\left(\mathrm{CH}_{2}\right), 107.9(\mathrm{CH}), 82.3(\mathrm{CH}), 65.3\left(\mathrm{CH}_{2}\right), 42.7\left(\mathrm{CH}_{2}\right), 40.5(\mathrm{C}), 25.7\left(\mathrm{CH}_{3}\right), 24.1\left(\mathrm{CH}_{3}\right)$, $21.31\left(\mathrm{CH}_{3}\right), 15.5\left(\mathrm{CH}_{3}\right)$;

IR (ATR) 2934, 2853, 1088, 1072, $1002 \mathrm{~cm}^{-1}$;

HRMS (APCl) m/z: [[M + H] $\left.-\mathrm{C}_{2} \mathrm{H}_{6} \mathrm{O}\right]^{+}$Calcd for $\mathrm{C}_{18} \mathrm{H}_{25} \mathrm{O}$ 273.2168; Found 273.2156.

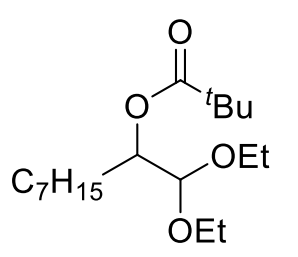

4b

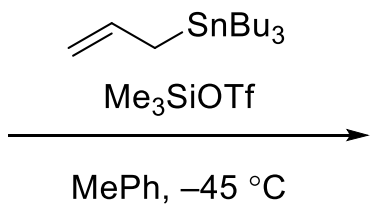

$15 b$<smiles>C=CC[C@@H](OCC)[C@@H]([18OH])OC(=O)C(C)(C)C</smiles>

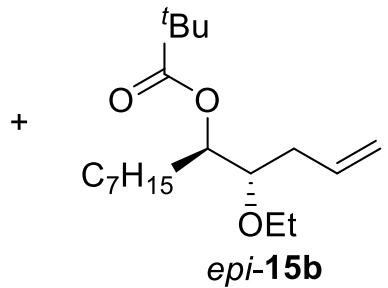

$\left(4 R^{\star}, 5 R^{\star}\right)$-4-Ethoxydodec-1-en-5-yl pivaloate $(15 \mathrm{~b})$ and $\left(4 R^{\star}, 5 S^{\star}\right)-4-e t h o x y d o d e c-1-e n-5$-yl pivaloate (epi-15b). Following the general procedure for acetal substitutions, acetal $\mathbf{4 b}(0.316 \mathrm{~g}$, $1.00 \mathrm{mmol})$ in MePh $(10 \mathrm{~mL})$ was mixed with allyltributylstannane $(1.22 \mathrm{~mL}, 4.00 \mathrm{mmol})$ and trimethylsilyl trifluoromethanesulfonate $(0.364 \mathrm{~mL}, 2.00 \mathrm{mmol})$ to give $15 \mathrm{~b}$ and epi-15b as a mixture of diastereomers (15b:epi-15b $=59: 41)$ as a colorless oil $(0.168 \mathrm{~g}, 55 \%)$. 15b and epi15b were isolated and characterized as an $87: 13$ mixture:

${ }^{1} \mathrm{H}$ NMR $\left(400 \mathrm{MHz}, \mathrm{CDCl}_{3}\right) \delta 5.78-5.88(\mathrm{~m}, 1.15 \mathrm{H}), 5.01-5.08(\mathrm{~m}, 2.3 \mathrm{H}), 4.92-4.96(\mathrm{~m}, 1.15 \mathrm{H})$, 3.54-3.63 (m, 2H), 3.45-3.51 (m, 0.3H), 3.31-3.38 (m, 1.15H), 2.20-2.25 (m, 2.3H), 1.54-1.61 $(\mathrm{m}, 2.3 \mathrm{H}), 1.25(\mathrm{br} \mathrm{s}, 13.8 \mathrm{H}), 1.20(\mathrm{~s}, 9 \mathrm{H}), 1.19(\mathrm{~s}, 1.17 \mathrm{H}), 1.15(\mathrm{t}, J=7.5,3.4 \mathrm{H}), 0.87(\mathrm{t}, J=6.8$, 3.4H);

Peaks in ${ }^{13} \mathrm{C}$ NMR $\left(100 \mathrm{MHz}, \mathrm{CDCl}_{3}\right)$ attributed to the major diastereomer (15b): $\delta 178.11(\mathrm{C})$, $135.3(\mathrm{CH}), 116.8\left(\mathrm{CH}_{2}\right), 79.5(\mathrm{CH}), 73.8(\mathrm{CH}), 65.86\left(\mathrm{CH}_{2}\right), 38.9(\mathrm{C}), 34.8\left(\mathrm{CH}_{2}\right), 31.9\left(\mathrm{CH}_{2}\right)$, $29.5\left(\mathrm{CH}_{2}\right), 29.2\left(\mathrm{CH}_{2}\right), 29.0\left(\mathrm{CH}_{2}\right), 27.29\left(\mathrm{CH}_{3}\right), 25.7\left(\mathrm{CH}_{2}\right), 22.7\left(\mathrm{CH}_{2}\right), 15.7\left(\mathrm{CH}_{3}\right), 14.17\left(\mathrm{CH}_{3}\right)$; 
Peaks in ${ }^{13} \mathrm{C} \mathrm{NMR}\left(100 \mathrm{MHz}, \mathrm{CDCl}_{3}\right)$ attributed to the minor diastereomer (epi-15b): $\delta 178.05(\mathrm{C})$, $135.1(\mathrm{CH}), 116.9\left(\mathrm{CH}_{2}\right), 80.4(\mathrm{CH}), 74.6(\mathrm{CH}), 65.95\left(\mathrm{CH}_{2}\right), 39.0(\mathrm{C}), 35.7\left(\mathrm{CH}_{2}\right), 31.8\left(\mathrm{CH}_{2}\right)$, $29.7\left(\mathrm{CH}_{2}\right), 29.4\left(\mathrm{CH}_{2}\right), 29.3\left(\mathrm{CH}_{2}\right), 27.27\left(\mathrm{CH}_{3}\right), 25.6\left(\mathrm{CH}_{2}\right), 22.5\left(\mathrm{CH}_{2}\right), 15.6\left(\mathrm{CH}_{3}\right), 14.15\left(\mathrm{CH}_{3}\right)$; IR (ATR) 2957, 2926, 1751, 911, $768 \mathrm{~cm}^{-1}$;

HRMS (APCl) m/z: [[M + H] $\left.-\mathrm{C}_{5} \mathrm{H}_{10} \mathrm{O}_{2}\right]^{+}$Calcd for $\mathrm{C}_{14} \mathrm{H}_{27} \mathrm{O} 211.2056$; Found 211.2053.

Anal. Calcd for $\mathrm{C}_{19} \mathrm{H}_{36} \mathrm{O}_{3}$ : C, 73.03; $\mathrm{H}, 11.61$. Found: $\mathrm{C}, 73.31 ; \mathrm{H}, 11.87$.<smiles>CCOC(OCC)C(OC(=O)Br)C1CCCCC1</smiles>

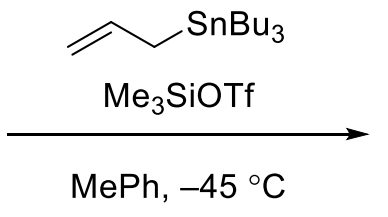<smiles>C=CC[C@H](OCC)C(OC(=O)CC(C)(C)C)C1CCCCC1</smiles><smiles>C=CC[C@H](OCC)C(OC(=O)CC(C)(C)C)C1CCCCC1</smiles>

$\left(1 R^{\star}, 2 R^{\star}\right)$-1-Cyclohexyl-2-ethoxypent-4-en-1-yl pivaloate (15e) and $\left(1 R^{\star}, 2 S^{\star}\right)-1$-cyclohexyl2-ethoxypent-4-en-1-yl pivaloate (epi-15e). Following the general procedure for acetal substitutions, acetal $4 \mathrm{e}(0.3 \mathrm{~g}, 1 \mathrm{mmol})$ in $\mathrm{MePh}(10 \mathrm{~mL})$ was mixed with allyltributylstannane $(1.22 \mathrm{~mL}, 4.00 \mathrm{mmol})$ and trimethylsilyl trifluoromethanesulfonate $(0.364 \mathrm{~mL}, 2.00 \mathrm{mmol})$ to give $15 \mathrm{e}$ and epi-15e as a mixture of diastereomers (15e:epi-15e $=82: 18)$ as a colorless oil $(0.172 \mathrm{~g}$, $58 \%$ ). 15e and epi-15e were isolated and characterized as a 75:25 mixture:

${ }^{1} \mathrm{H}$ NMR $\left(400 \mathrm{MHz}, \mathrm{CDCl}_{3}\right) \delta 5.75-5.83(\mathrm{~m}, 1.3 \mathrm{H}), 5.03$ (dd overlapped, $\left.J=15.7,8.7,2.7 \mathrm{H}\right), 4.88$ $(\mathrm{t}, J=5.8,0.3 \mathrm{H}), 4.75(\mathrm{t}, J=5.5,1 \mathrm{H}), 3.50-3.59(\mathrm{~m}, 1.3 \mathrm{H}), 3.38-3.49(\mathrm{~m}, 2.6 \mathrm{H}), 2.17-2.25(\mathrm{~m}$, 2.6H), 1.60-1.70 (br dd, $J=18.1,11.7,10 \mathrm{H}), 1.19(\mathrm{~s}, 9 \mathrm{H}), 1.18(\mathrm{~s}, 3 \mathrm{H}), 1.12(\mathrm{t}, J=6.9,3.75 \mathrm{H})$, 0.97-1.04 (m, 2.6H);

Peaks in ${ }^{13} \mathrm{C}$ NMR $\left(100 \mathrm{MHz}, \mathrm{CDCl}_{3}\right)$ attributed to the major diastereomer (15e): $\delta 177.9$ (C), $134.8(\mathrm{CH}), 117.1\left(\mathrm{CH}_{2}\right), 78.6(\mathrm{CH}), 77.3(\mathrm{CH}), 65.4\left(\mathrm{CH}_{2}\right), 39.03(\mathrm{C}), 38.2(\mathrm{CH}), 35.1\left(\mathrm{CH}_{2}\right)$, $29.97\left(\mathrm{CH}_{2}\right), 27.9\left(\mathrm{CH}_{2}\right), 27.39\left(\mathrm{CH}_{3}\right), 26.42\left(\mathrm{CH}_{2}\right), 26.16\left(\mathrm{CH}_{2}\right), 26.0\left(\mathrm{CH}_{2}\right), 15.6\left(\mathrm{CH}_{3}\right)$;

Peaks in ${ }^{13} \mathrm{C}$ NMR $\left(100 \mathrm{MHz}, \mathrm{CDCl}_{3}\right)$ attributed to the minor diastereomer (epi-15e): $\delta 177.8$ (C), $135.4(\mathrm{CH}), 116.6\left(\mathrm{CH}_{2}\right), 78.0(\mathrm{CH}), 76.1(\mathrm{CH}), 65.0\left(\mathrm{CH}_{2}\right), 39.01(\mathrm{C}), 38.4(\mathrm{CH}), 34.5\left(\mathrm{CH}_{2}\right)$, $29.91\left(\mathrm{CH}_{2}\right), 28.0\left(\mathrm{CH}_{2}\right), 27.36\left(\mathrm{CH}_{3}\right), 27.2\left(\mathrm{CH}_{2}\right), 26.44\left(\mathrm{CH}_{2}\right), 26.18\left(\mathrm{CH}_{2}\right), 15.5\left(\mathrm{CH}_{3}\right)$; IR (ATR) 2973, 2927, 1727, 911, $765 \mathrm{~cm}^{-1}$; HRMS (APCI) m/z: [[M + H] $\left.-\mathrm{C}_{5} \mathrm{H}_{10} \mathrm{O}_{2}\right]^{+}$Calcd for $\mathrm{C}_{13} \mathrm{H}_{23} \mathrm{O}$ 195.1743; Found 195.1741. Anal. Calcd for $\mathrm{C}_{18} \mathrm{H}_{32} \mathrm{O}_{3}$ : C, 72.93; $\mathrm{H}, 10.88$. Found: $\mathrm{C}, 73.16 ; \mathrm{H}, 11.00$.<smiles>CCOC(OCC)C(Br)OC(=O)Br</smiles>

4d

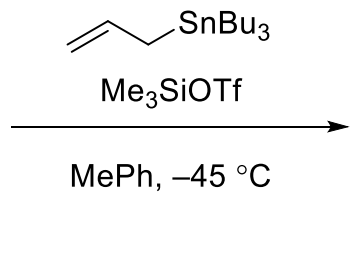<smiles>C=CCC(OC(C)(C)C)C(Br)OC(=O)CC</smiles>

$15 d$<smiles>C=CC[C@H](OC(=O)C(C)(C)C)[C@@H](CC=C)O[Ga]</smiles>
$(15 d), \quad\left(3 R^{\star}, 4 S^{\star}\right)-4-e t h o x y-2,2-$ dimethylhept-6-en-3-yl pivaloate $(e p i-15 \mathrm{~d})$, and $\left(2 R^{\star}, 4 R^{\star}, 5 S^{\star}\right)$-2-allyl-2,4-di-tert-butyl-5ethoxy-1,3-dioxolane (S40). Following the general procedure for acetal substitutions, acetal 4d $(0.274 \mathrm{~g}, 1.00 \mathrm{mmol})$ in $\mathrm{MePh}(10 \mathrm{~mL})$ was mixed with allyltributylstannane $(1.22 \mathrm{~mL}, 4.00 \mathrm{mmol})$ and trimethylsilyl trifluoromethanesulfonate $(0.364 \mathrm{~mL}, 2.00 \mathrm{mmol})$ to give an 80:20 mixture of 15d and epi-15d to S40, where 15d and epi-15d were formed as a mixture of diastereomers (15d:epi-15d = 85:15), and dioxolane $\mathbf{S 4 0}$ was formed as a single diastereomer. The products were isolated as a 77:23 mixture of $\mathbf{1 5 d}$, epi-15d and dioxolane $\mathbf{S 4 0}$ as a colorless oil $(0.186 \mathrm{~g}$, $68 \%$ ). 15d and epi-15d were characterized as a 94:6 mixture of diastereomers and dioxolane $\mathbf{S 4 0}$ 
was characterized as a single diastereomer. All analytical data was collected using the 77:23 mixture of products.

Peaks in ${ }^{1} \mathrm{H}$ NMR $\left(400 \mathrm{MHz}, \mathrm{CDCl}_{3}\right.$ ) attributed to $\mathbf{1 5 d}$ and epi-15d: $\delta 5.81$ (ddt, $J=17.2,10.1$, $7.1,1.06 \mathrm{H}), 5.00-5.09(\mathrm{~m}, 2.12 \mathrm{H}), 4.88(\mathrm{~d}, J=4.1,0.06 \mathrm{H}), 4.61(\mathrm{~d}, J=2.9,1 \mathrm{H}), 3.55-3.64(\mathrm{~m}$, $1.06 \mathrm{H}$ ), $3.41-3.56(\mathrm{~m}, 1.06 \mathrm{H}), 2.33$ (quint, $J=6.7,1.06 \mathrm{H}), 2.08$ (quint, $J=6.7,1.06 \mathrm{H}), 1.25(\mathrm{~s}$, $9.54 \mathrm{H}), 1.16(\mathrm{t}, J=7.3,3.18 \mathrm{H}), 0.96(\mathrm{~s}, 9.54 \mathrm{H})$;

Peaks in ${ }^{13} \mathrm{C}$ NMR $\left(100 \mathrm{MHz}, \mathrm{CDCl}_{3}\right)$ attributed to the major diastereomer (15d): $\delta 178.1$ (C), $135.2(\mathrm{CH}), 117.3\left(\mathrm{CH}_{2}\right), 79.4(\mathrm{CH}), 78.4(\mathrm{CH}), 64.9\left(\mathrm{CH}_{2}\right), 39.3(\mathrm{C}), 36.7\left(\mathrm{CH}_{2}\right), 32.6(\mathrm{C}), 27.5$ $\left(\mathrm{CH}_{3}\right), 27.1\left(\mathrm{CH}_{3}\right), 15.7\left(\mathrm{CH}_{3}\right)$;

Peaks in $\left.{ }^{13} \mathrm{C} \mathrm{NMR} \mathrm{(100} \mathrm{MHz,} \mathrm{CDCl}_{3}\right)$ attributed to the minor diastereomer (epi-15d): $\delta 177.5(\mathrm{C})$, $135.8(\mathrm{CH}), 114.1\left(\mathrm{CH}_{2}\right), 79.1(\mathrm{CH}), 79.0(\mathrm{CH}), 64.3\left(\mathrm{CH}_{2}\right), 39.2(\mathrm{C}), 35.6\left(\mathrm{CH}_{2}\right), 33.9(\mathrm{C}), 27.0$ $\left(\mathrm{CH}_{3}\right), 26.9\left(\mathrm{CH}_{3}\right), 15.5\left(\mathrm{CH}_{3}\right)$;

IR (ATR) 2960, 1727, 1480, 1281, $1074 \mathrm{~cm}^{-1}$;

HRMS (APCl) m/z: [[M + H] $\left.-\mathrm{C}_{5} \mathrm{H}_{10} \mathrm{O}_{2}\right]^{+}$Calcd for $\mathrm{C}_{11} \mathrm{H}_{21} \mathrm{O}$ 169.1587; Found 169.1584; $\mathrm{m} / \mathrm{z}$ : ((M $+\mathrm{H})-\mathrm{C}_{2} \mathrm{H}_{6} \mathrm{O}$ ) ${ }^{+}$Calcd for $\mathrm{C}_{14} \mathrm{H}_{25} \mathrm{O}_{2}$ 225.1849; Found 225.1849 (note: this peak could be formed from any of the three products).

Peaks in ${ }^{1} \mathrm{H}$ NMR $\left(400 \mathrm{MHz}, \mathrm{CDCl}_{3}\right)$ attributed to dioxolane (S40): $\delta 5.89-5.99(\mathrm{~m}, 0.3 \mathrm{H}), 4.95$ $(\mathrm{d}, J=10.2,0.6 \mathrm{H}), 4.79(\mathrm{~d}, J=5.1,0.3 \mathrm{H}), 3.78-3.86(\mathrm{~m}, 0.3 \mathrm{H}), 2.68(\mathrm{dq}, J=15.3,6.7,0.6 \mathrm{H})$, $1.60-1.68(\mathrm{~m}, 0.3 \mathrm{H}), 1.29-1.41(\mathrm{~m}, 0.3 \mathrm{H}), 1.17-1.21(\mathrm{~m}, 0.9 \mathrm{H}), 0.97(\mathrm{~s}, 2.7 \mathrm{H}), 0.94(\mathrm{~s}, 2.7 \mathrm{H})$;

Peaks in ${ }^{13} \mathrm{C} \mathrm{NMR}\left(100 \mathrm{MHz}, \mathrm{CDCl}_{3}\right)$ attributed to dioxolane (S40): $\delta 136.3(\mathrm{CH}), 116.5(\mathrm{C}), 115.3$ $\left(\mathrm{CH}_{2}\right), 102.9(\mathrm{CH}), 88.5(\mathrm{CH}), 53.5\left(\mathrm{CH}_{2}\right), 39.6(\mathrm{C}), 35.0\left(\mathrm{CH}_{2}\right), 34.6(\mathrm{C}), 26.3\left(\mathrm{CH}_{3}\right), 26.0\left(\mathrm{CH}_{3}\right)$, $13.7\left(\mathrm{CH}_{3}\right)$.<smiles>CCOC(OCC)[C@H](Cc1ccccc1)OC(=O)Br</smiles>

$(R)-\mathbf{4 f}$

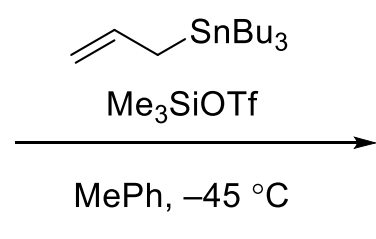

$(2 R, 3 R)-3-E t h o x y-1-p h e n y l h e x-5-e n-2-y$

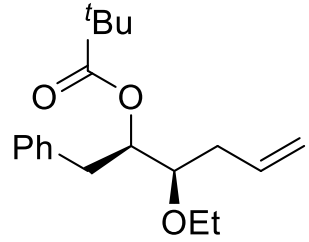

$(R)-15 f$



epi-(R)-15f phenylhex-5-en-2-yl pivaloate $(e p i-(R)-15 f)$. Following the general procedure for acetal substitutions, acetal $(R)-\mathbf{4 f}(0.31 \mathrm{~g}, 1.0 \mathrm{mmol})$ in MePh $(10 \mathrm{~mL})$ was combined with allyltributylstannane $(1.22 \mathrm{~mL}, 4.00 \mathrm{mmol})$ and trimethylsilyl trifluoromethanesulfonate $(0.364 \mathrm{~mL}$,

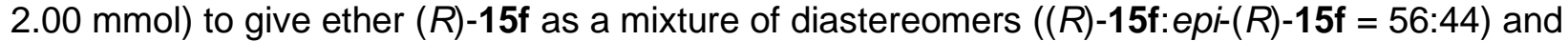
as a colorless oil $(0.117 \mathrm{~g}, 34 \%, 50 \% \mathrm{brsm})$. $(R)-\mathbf{1 5 f}$ and epi- $(R)-\mathbf{1 5 f}$ were isolated and characterized as a 62:38 mixture:

${ }^{1} \mathrm{H} \mathrm{NMR}\left(400 \mathrm{MHz}, \mathrm{CDCl}_{3}\right) \delta$ 7.23-7.27 (m, 3.2H), 7.15-7.21 (m, 5H), 5.78-5.92 (m, 1.6H), 5.15$5.19(\mathrm{~m}, 1.6 \mathrm{H}), 5.04-5.12(\mathrm{~m}, 3.2 \mathrm{H}), 3.49-3.66(\mathrm{~m}, 3.2 \mathrm{H}), 3.43-3.452(\mathrm{~m}, 0.6 \mathrm{H}), 3.36-3.41(\mathrm{~m}$, $1 \mathrm{H}), 2.77-3.06(\mathrm{~m}, 3.2 \mathrm{H}), 2.29-2.33(\mathrm{~m}, 3.2 \mathrm{H}), 1.21(\mathrm{dt}, J=10.3,6.8,4.8 \mathrm{H}), 1.09(\mathrm{~s}, 9 \mathrm{H}), 1.06$ $(\mathrm{s}, 6 \mathrm{H})$;

Peaks in ${ }^{13} \mathrm{C}$ NMR $\left(100 \mathrm{MHz}, \mathrm{CDCl}_{3}\right)$ attributed to the major diastereomer $((R)-15 f): \delta 177.8(\mathrm{C})$, $138.0(\mathrm{C}), 135.0(\mathrm{CH}), 129.4(\mathrm{CH}), 128.4(\mathrm{CH}), 126.42(\mathrm{CH}), 117.1\left(\mathrm{CH}_{2}\right), 78.7(\mathrm{CH}), 74.6(\mathrm{CH})$, $65.8\left(\mathrm{CH}_{2}\right)$, $38.84(\mathrm{C}), 35.5\left(\mathrm{CH}_{2}\right), 34.7\left(\mathrm{CH}_{2}\right), 27.18\left(\mathrm{CH}_{3}\right), 15.8\left(\mathrm{CH}_{3}\right)$;

Peaks in ${ }^{13} \mathrm{C}$ NMR $\left(100 \mathrm{MHz}, \mathrm{CDCl}_{3}\right)$ attributed to the minor diastereomer $(e p i-(R)-15): \delta 177.7$

$(\mathrm{C}), 137.9(\mathrm{C}), 134.7(\mathrm{CH}), 129.5(\mathrm{CH}), 128.3(\mathrm{CH}), 126.41(\mathrm{CH}), 117.2\left(\mathrm{CH}_{2}\right), 79.7(\mathrm{CH}), 75.3$

$(\mathrm{CH}), 66.0\left(\mathrm{CH}_{2}\right), 38.79(\mathrm{C}), 35.8\left(\mathrm{CH}_{2}\right), 35.6\left(\mathrm{CH}_{2}\right), 27.15\left(\mathrm{CH}_{3}\right), 15.7\left(\mathrm{CH}_{3}\right)$;

IR (ATR) 2973, 1726, 1107, 912, $745 \mathrm{~cm}^{-1}$;

$[\alpha]_{\mathrm{D}}^{\mathrm{rt}}=-2.2405^{\circ}\left(\mathrm{c}=1.00, \mathrm{CHCl}_{3}\right)$ 
HRMS (APCI) m/z: [[M + H] $\left.-\mathrm{C}_{2} \mathrm{H}_{6} \mathrm{O}\right]^{+}$Calcd for $\mathrm{C}_{17} \mathrm{H}_{24} \mathrm{O}_{2}$ 259.1693; Found 259.1690. Anal. Calcd for $\mathrm{C}_{19} \mathrm{H}_{28} \mathrm{O}_{3}$ : C, 74.96; $\mathrm{H}, 9.27$. Found: $\mathrm{C}, 74.80 ; \mathrm{H}, 9.36$.<smiles>CCOC(OCC)C(Cc1ccccc1)OC(=O)Br</smiles>

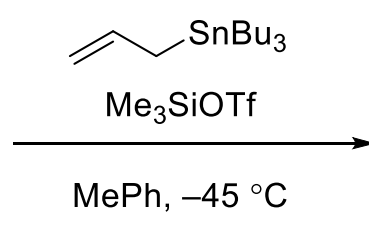<smiles>C=CC[C@H](OC(Br)(Br)Br)[C@H](Cc1ccccc1)OC(=O)CC</smiles>

$15 f$<smiles>C=CCC(OCC)C(Cc1ccccc1)OC(=O)CC(C)(C)C</smiles>

epi-15f

$\left(2 R^{\star}, 3 R^{\star}\right)$-3-Ethoxy-1-phenylhex-5-en-2-yl pivaloate $((\mathrm{rac})-15 \mathrm{f})$ and $\left(2 R^{\star}, 3 S^{\star}\right)$-3-ethoxy-1phenylhex-5-en-2-yl pivaloate (epi-15f). Following the general procedure for acetal substitutions, acetal (rac)-4f $(0.154 \mathrm{~g}, 0.500 \mathrm{mmol})$ in $\mathrm{MePh}(5 \mathrm{~mL})$ was combined with allyltributylstannane $(0.61 \mathrm{~mL}, 2.0 \mathrm{mmol})$ and trimethylsilyl trifluoromethanesulfonate $(0.182 \mathrm{~mL}$, $1.00 \mathrm{mmol})$ to give $(\mathrm{rac})-\mathbf{1 5 f}$ as a mixture of diastereomers $((\mathrm{rac})-\mathbf{1 5 f}$ : $(\mathrm{rac})$-epi-15f $=\mathbf{5 6 : 4 4 )}$ and as a colorless oil $(0.129 \mathrm{~g}, 85 \%)$. The spectral data obtained are consistent with those reported above for the enantioenriched compound.<smiles>CCOC(OCC)C(OC(=O)CC(C)C)c1cccc2ccccc12</smiles>

4c

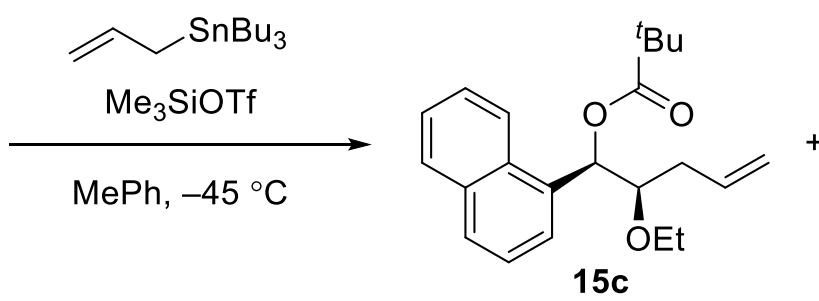

$15 \mathrm{c}$<smiles>C=CC[C@H](OCC)c1cccc2ccccc12</smiles>

epi-15c

$\left(1 R^{\star}, 2 R^{\star}\right)$-2-Ethoxy-1-(naphthalen-1-yl)pent-4-en-1-yl pivaloate $(15 \mathrm{c})$ and $\left(1 R^{\star}, 2 S^{\star}\right)-2-$ ethoxy-1-(naphthalen-1-yl)pent-4-en-1-yl pivaloate (epi-15c). Following the general procedure for acetal substitutions, acetal $4 \mathrm{c}(0.13 \mathrm{~g}, 0.34 \mathrm{mmol})$ in $\mathrm{MePh}(4 \mathrm{~mL})$ was mixed with allyltributylstannane $(1.22 \mathrm{~mL}, 4.00 \mathrm{mmol})$ and trimethylsilyl trifluoromethanesulfonate $(0.364 \mathrm{~mL}$, $2.00 \mathrm{mmol}$ ) to give $15 \mathrm{c}$ as a mixture of diastereomers (15c:epi-15c $=88: 12$ ) and as a colorless oil $(0.106 \mathrm{~g}, 83 \%)$. The diastereomers were separated by column chromatography and characterized by ${ }^{1} \mathrm{H}$ and ${ }^{13} \mathrm{C}$ NMR separately, and IR, HRMS, and elemental analysis are reported for the major diastereomer.

Major diastereomer (15c): ${ }^{1} \mathrm{H}$ NMR $\left(400 \mathrm{MHz}, \mathrm{CDCl}_{3}\right) \delta 8.27(\mathrm{~d}, J=8.4,1 \mathrm{H}), 7.87(\mathrm{~d}, J=8.4$, $1 \mathrm{H}), 7.82(\mathrm{~d}, J=8.4,1 \mathrm{H}), 7.45-7.60(\mathrm{~m}, 4 \mathrm{H}), 6.50(\mathrm{~d}, J=6.7,1 \mathrm{H}), 5.81$ (ddt, $J=17.2,10.3,7.1$, $1 \mathrm{H}), 5.03(\mathrm{~d}, J=10.1,1 \mathrm{H}), 4.98(\mathrm{~d}, J=16.8,1 \mathrm{H}), 3.87(\mathrm{q}, J=6.7,1 \mathrm{H}), 3.46-3.57(\mathrm{~m}, 2 \mathrm{H}), 2.16-$ $2.23(\mathrm{~m}, 1 \mathrm{H}), 2.07-2.13(\mathrm{~m}, 1 \mathrm{H}), 1.27(\mathrm{~s}, 9 \mathrm{H}), 1.11(\mathrm{t}, J=6.9,3 \mathrm{H})$;

${ }^{13} \mathrm{C}$ NMR (100 MHz, CDCl $\left.{ }_{3}\right) \delta 177.5(\mathrm{C}), 134.5(\mathrm{CH}), 134.4(\mathrm{C}), 133.9(\mathrm{C}), 131.0(\mathrm{C}), 128.93(\mathrm{CH})$, $128.7(\mathrm{CH}), 126.3(\mathrm{CH}), 125.70(\mathrm{CH}), 125.66(\mathrm{CH}), 125.2(\mathrm{CH}), 123.7(\mathrm{CH}), 117.5\left(\mathrm{CH}_{2}\right), 81.47$ $(\mathrm{CH}), 74.8(\mathrm{CH}), 67.2\left(\mathrm{CH}_{2}\right), 38.9(\mathrm{C}), 36.4\left(\mathrm{CH}_{2}\right), 27.28\left(\mathrm{CH}_{3}\right), 15.5\left(\mathrm{CH}_{3}\right)$;

IR (ATR) 2974, 1729, 1103, 914, $734 \mathrm{~cm}^{-1}$;

HRMS (APCl) m/z: [[M + H] $\left.-\mathrm{C}_{5} \mathrm{H}_{10} \mathrm{O}_{2}\right]^{+}$Calcd for $\mathrm{C}_{17} \mathrm{H}_{19} \mathrm{O} 239.1430$; Found 239.1430.

Anal. Calcd for $\mathrm{C}_{22} \mathrm{H}_{28} \mathrm{O}_{3}$ : $\mathrm{C}, 77.61 ; \mathrm{H}, 8.29$. Found: $\mathrm{C}, 77.37 ; \mathrm{H}, 8.36$.

Minor diastereomer (epi-15c): ${ }^{1} \mathrm{H}$ NMR $\left(400 \mathrm{MHz}, \mathrm{CDCl}_{3}\right) \delta 8.21(\mathrm{~d}, J=8.6,1 \mathrm{H}), 7.87(\mathrm{~d}, J=7.9$, $1 \mathrm{H}), 7.81(\mathrm{~d}, J=7.9,1 \mathrm{H}), 7.60(\mathrm{~d}, J=7.9,1 \mathrm{H}), 7.45-7.57(\mathrm{~m}, 3 \mathrm{H}), 6.65(\mathrm{~d}, J=5.4,1 \mathrm{H}), 5.81-$ $5.91(\mathrm{~m}, 1 \mathrm{H}), 5.02-5.06(\mathrm{~m}, 2 \mathrm{H}), 3.78-3.83(\mathrm{~m}, 1 \mathrm{H}), 3.33-3.45(\mathrm{~m}, 2 \mathrm{H}), 2.35-2.39(\mathrm{~m}, 2 \mathrm{H}), 1.28$ $(\mathrm{s}, 9 \mathrm{H}), 0.95(\mathrm{t}, J=6.8,3 \mathrm{H})$; 
${ }^{13} \mathrm{C} \mathrm{NMR}\left(100 \mathrm{MHz}, \mathrm{CDCl}_{3}\right) \delta 177.3(\mathrm{C}), 135.2(\mathrm{CH}), 134.6(\mathrm{C}), 133.8(\mathrm{C}), 131.1(\mathrm{C}), 128.91(\mathrm{CH})$, $128.5(\mathrm{CH}), 126.2(\mathrm{CH}), 125.69(\mathrm{CH}), 125.3(\mathrm{CH}), 124.9(\mathrm{CH}), 123.6(\mathrm{CH}), 116.9\left(\mathrm{CH}_{2}\right), 81.48$ $(\mathrm{CH}), 72.5(\mathrm{CH}), 66.2\left(\mathrm{CH}_{2}\right), 39.1(\mathrm{C}), 35.4\left(\mathrm{CH}_{2}\right), 27.34\left(\mathrm{CH}_{3}\right), 15.4\left(\mathrm{CH}_{3}\right)$.

\section{Cyclization of substitution products}

General procedure for ester reduction. This procedure was adapted from that reported by Rein et. al. ${ }^{10}$ To a cooled $\left(0{ }^{\circ} \mathrm{C}\right)$ solution of the ether (1 equiv) in $\mathrm{Et}_{2} \mathrm{O}(0.1 \mathrm{M})$ was added $\mathrm{LiAlH}_{4}(1.5$ equiv). The reaction mixture was warmed to room temperature over $1 \mathrm{~h}$. After $1 \mathrm{~h}$, the reaction mixture was diluted with EtOAc ( $2 \mathrm{~mL}$ per $0.05 \mathrm{mmol})$, and then a $1: 1$ solution $(\mathrm{v} / \mathrm{v})$ of $\mathrm{H}_{2} \mathrm{O}: \mathrm{MeOH}$ $(5 \mathrm{~mL}$ per $0.05 \mathrm{mmol}$ ) was added. The mixture was stirred for $5 \mathrm{~h}$ until it turned clear, and the aqueous layer was extracted with EtOAc $(2 \times 5 \mathrm{~mL}$ per $0.05 \mathrm{mmol})$. The organic layers were combined and washed with $\mathrm{H}_{2} \mathrm{O}(5 \mathrm{~mL}$ per $0.05 \mathrm{mmol})$ and then brine $(5 \mathrm{~mL}$ per $0.05 \mathrm{mmol})$, dried over $\mathrm{Na}_{2} \mathrm{SO}_{4}$, filtered through cotton, and concentrated in vacuo to provide the alcohol, which was used without further purification. Sufficient material to collect IR or HRMS data for some compounds was not obtained.

General procedure for cyclization of alcohols. This procedure was adapted by Tony et. al. ${ }^{10}$ To a solution of alcohol (1 equiv) in $\mathrm{CH}_{3} \mathrm{CN}$ was added NIS (4 equiv) and $\mathrm{AgOTf}$ ( 0.2 equiv). After $30 \mathrm{~min}$, a saturated solution of $\mathrm{Na}_{2} \mathrm{~S}_{2} \mathrm{O}_{3}$ was added $(5 \mathrm{~mL}$ per $0.05 \mathrm{mmol})$, and the aqueous layer was extracted with EtOAc $(2 \times 10 \mathrm{~mL}$ per $0.050 \mathrm{mmol})$. The combined organic layers were washed brine $(10 \mathrm{~mL}$ per $0.050 \mathrm{mmol})$, dried over $\mathrm{Na}_{2} \mathrm{SO}_{4}$, filtered through cotton, and concentrated in vacuo. The diastereomeric ratio was determined by NMR analysis $\left({ }^{1} \mathrm{H}\right.$ and $\left.{ }^{13} \mathrm{C}\right)$ of the unpurified products. The residue was purified by column chromatography (5:95 EtOAc:hexanes) to provide the product.



$\left(2 R^{\star}, 3 R^{\star}, 5 R^{\star}\right)$-3-Ethoxy-5-(iodomethyl)-2-(p-tolyl)tetrahydrofuran (S42) and $\left(2 R^{\star}, 3 R^{\star}, 5 S^{\star}\right)$ 3-ethoxy-5-(iodomethyl)-2-( $p$-tolyl)tetrahydrofuran (epi-S42). Following the general procedure for reduction, $15 \mathrm{a}(0.06 \mathrm{~g}, 0.2 \mathrm{mmol})$ in $\mathrm{Et}_{2} \mathrm{O}(2 \mathrm{~mL})$ was mixed with $\mathrm{LiAlH}_{4}(0.015 \mathrm{~g}$, $0.40 \mathrm{mmol})$ to provide alcohol $\mathbf{S 4 1}$ as a colorless oil $(0.039 \mathrm{~g}, 89 \%)$ :

${ }^{1} \mathrm{H}$ NMR $\left(400 \mathrm{MHz}, \mathrm{CDCl}_{3}\right) \delta 7.26$ (d, $\left.J=7.9,2 \mathrm{H}\right), 7.16$ (d, $\left.J=7.9,2 \mathrm{H}\right), 5.80$ (ddt, $J=17.1,7.7$, $6.9,1 \mathrm{H}), 5.02-5.07(\mathrm{~m}, 2 \mathrm{H}), 4.51(\mathrm{dd}, J=7.2,2.7,1 \mathrm{H}), 3.66-3.74(\mathrm{~m}, 1 \mathrm{H}), 3.41-3.51(\mathrm{~m}, 2 \mathrm{H})$, $3.05(\mathrm{~d}, J=2.5,1 \mathrm{H}), 2.35(\mathrm{~s}, 3 \mathrm{H}), 2.27-2.33(\mathrm{~m}, 1 \mathrm{H}), 2.05-2.12(\mathrm{~m}, 1 \mathrm{H}), 1.22(\mathrm{t}, J=6.9,3 \mathrm{H})$;

${ }^{13} \mathrm{C}$ NMR (100 MHz, CDCl $)$ ס $137.9(\mathrm{C}), 137.7(\mathrm{C}), 134.3(\mathrm{CH}), 129.2(\mathrm{CH}), 127.2(\mathrm{CH}), 117.5$ $\left(\mathrm{CH}_{2}\right)$, 83.6 $(\mathrm{CH}), 75.5(\mathrm{CH}), 66.1\left(\mathrm{CH}_{2}\right), 34.9\left(\mathrm{CH}_{2}\right), 21.3\left(\mathrm{CH}_{3}\right), 15.7\left(\mathrm{CH}_{3}\right)$;

IR (ATR) 3680, 2980, 2844, 1109, $1056 \mathrm{~cm}^{-1}$.

Following the general procedure for cyclization, alcohol $\mathbf{S} 41(0.01 \mathrm{~g}, 0.05 \mathrm{mmol})$ in $\mathrm{CH}_{3} \mathrm{CN}(4 \mathrm{~mL})$ was mixed with NIS $(0.041 \mathrm{~g}, 0.18 \mathrm{mmol})$ and AgOTf $(0.002 \mathrm{~g}, 0.008 \mathrm{mmol})$ to provide the product as a 60:40 mixture of diastereomers and as a colorless oil $(0.015 \mathrm{~g}, 94 \%)$. lodofurans $\mathbf{S 4 2}$ and epi-S42 were isolated and characterized as a 60:40 mixture, but the material decomposed before IR data was collected: 
${ }^{1} \mathrm{H}$ NMR $\left(600 \mathrm{MHz}, \mathrm{CDCl}_{3}\right) \delta 7.27-7.30(\mathrm{~m}, 3.12 \mathrm{H}), 7.13-7.15(\mathrm{~m}, 3.12 \mathrm{H}), 5.06(\mathrm{~d}, \mathrm{~J}=3.4,1 \mathrm{H})$, $4.87(\mathrm{~d}, J=3.8,0.6 \mathrm{H}), 4.40-4.46(\mathrm{~m}, 1 \mathrm{H}), 4.29-4.35(\mathrm{~m}, 0.6 \mathrm{H}), 4.05(\mathrm{t}, J=3.5,1 \mathrm{H}), 4.00(\mathrm{t}, J=$ $4.4,0.6 \mathrm{H}), 3.34-3.52(\mathrm{~m}, 3.2 \mathrm{H}), 3.17-3.26(\mathrm{~m}, 1.6 \mathrm{H}), 2.83-2.92(\mathrm{~m}, 1.6 \mathrm{H}), 2.37-2.44(\mathrm{~m}, 1.6 \mathrm{H})$, $2.36(\mathrm{~s}, 1.8 \mathrm{H}), 2.34(\mathrm{~s}, 3 \mathrm{H}), 2.16$ (dddd, $J=17.9,13.7,4.1,1.8,0.6 \mathrm{H}), 1.94-2.01(\mathrm{~m}, 1 \mathrm{H}), 1.96-$ $2.00(\mathrm{~m}, 1 \mathrm{H}), 0.92-0.94(\mathrm{~m}, 4.8 \mathrm{H})$;

Peaks in ${ }^{13} \mathrm{C}$ NMR $\left(100 \mathrm{MHz}, \mathrm{CDCl}_{3}\right.$ ) attributed to the major diastereomer (S42): $\delta 137.1$ (C), $134.7(\mathrm{C}), 128.6(\mathrm{CH}), 127.4(\mathrm{CH}), 85.2(\mathrm{CH}), 82.3(\mathrm{CH}), 77.3(\mathrm{CH}), 65.8\left(\mathrm{CH}_{2}\right), 40.6\left(\mathrm{CH}_{2}\right), 22.8$ $\left(\mathrm{CH}_{3}\right), 14.3\left(\mathrm{CH}_{3}\right), 11.9\left(\mathrm{CH}_{2}\right)$;

Peaks in ${ }^{13} \mathrm{C} \mathrm{NMR}\left(100 \mathrm{MHz}, \mathrm{CDCl}_{3}\right)$ attributed to the minor diastereomer (epi-S42): $\delta 137.2$ (C), $134.4(\mathrm{C}), 128.5(\mathrm{CH}), 127.6(\mathrm{CH}), 86.2(\mathrm{CH}), 80.9(\mathrm{CH}), 78.4(\mathrm{CH}), 65.6\left(\mathrm{CH}_{2}\right), 38.7\left(\mathrm{CH}_{2}\right), 21.4$ $\left(\mathrm{CH}_{3}\right), 15.2\left(\mathrm{CH}_{3}\right), 10.2\left(\mathrm{CH}_{2}\right)$;

HRMS (ESI) m/z: [M + Na] ${ }^{+}$Calcd for $\mathrm{C}_{14} \mathrm{H}_{19} \mathrm{INaO}_{2}$ 369.0340; Found 369.0346.

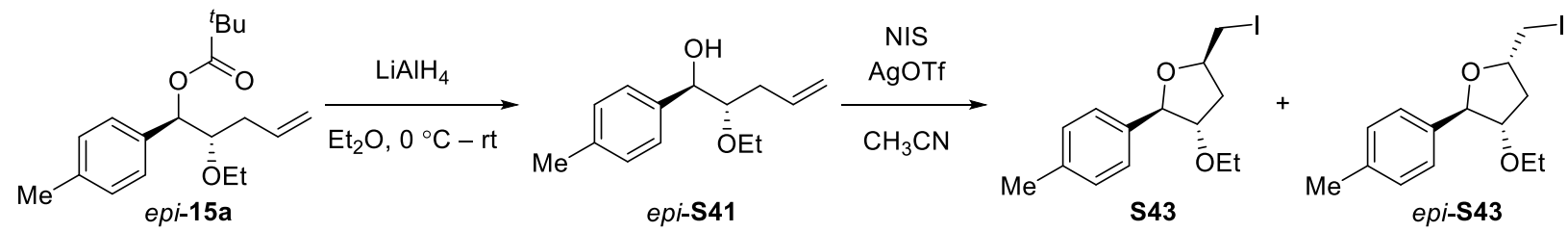

$\left(2 R^{\star}, 3 S^{\star}, 5 R^{\star}\right)$-3-Ethoxy-5-(iodomethyl)-2-( $p$-tolyl)tetrahydrofuran (S43) and $\left(2 R^{\star}, 3 S^{\star}, 5 S^{\star}\right)-3-$ ethoxy-5-(iodomethyl)-2-(p-tolyl)tetrahydrofuran (epi-S43). Following the general procedure for reduction, epi-15a $(0.01 \mathrm{~g}, 0.03 \mathrm{mmol})$ in $\mathrm{Et}_{2} \mathrm{O}(0.25 \mathrm{~mL})$ was mixed with $\mathrm{LiAlH}_{4}(0.002 \mathrm{~g}, 0.05$ $\mathrm{mmol})$ to provide alcohol epi-S41 as a colorless oil $(0.008 \mathrm{~g}, 99 \%)$ :

${ }^{1} \mathrm{H}$ NMR: $\left(400 \mathrm{MHz}, \mathrm{CDCl}_{3}\right) \delta 7.25(\mathrm{~d}, J=8.1,2 \mathrm{H}), 7.16(\mathrm{~d}, J=8.1,2 \mathrm{H}), 5.81$ (ddt, $J=16.9,10.2$, 6.8, 1H), 4.99-5.04 (m, 2H), $4.84(\mathrm{t}, J=3.3,1 \mathrm{H}), 3.56(\mathrm{q}, J=7.3,2 \mathrm{H}), 3.46-3.50(\mathrm{~m}, 1 \mathrm{H}), 2.46$ (br s, $1 \mathrm{H}), 2.35(\mathrm{~s}, 3 \mathrm{H}), 2.23-2.33(\mathrm{~m}, 1 \mathrm{H}), 1.18(\mathrm{t}, J=6.9,3 \mathrm{H})$;

${ }^{13} \mathrm{C}$ NMR (100 MHz, CDCl $\left.{ }_{3}\right) \delta 137.8(\mathrm{C}), 137.2(\mathrm{C}), 135.7(\mathrm{CH}), 129.1(\mathrm{CH}), 126.5(\mathrm{CH}), 116.7$ $\left(\mathrm{CH}_{2}\right)$, $83.3(\mathrm{CH}), 74.3(\mathrm{CH}), 65.9\left(\mathrm{CH}_{2}\right), 33.8\left(\mathrm{CH}_{2}\right), 21.3\left(\mathrm{CH}_{3}\right), 15.6\left(\mathrm{CH}_{3}\right)$.

Following the general procedure for cyclization, alcohol epi-S41 $(0.005 \mathrm{~g}, 0.02 \mathrm{mmol})$ in $\mathrm{CH}_{3} \mathrm{CN}$ $(2 \mathrm{~mL})$ was mixed with NIS $(0.021 \mathrm{~g}, 0.091 \mathrm{mmol})$ and AgOTf $(0.002 \mathrm{~g}, 0.005 \mathrm{mmol})$ to provide the product as a 61:39 mixture of diastereomers of the product as a colorless oil $(0.008 \mathrm{~g}, 99 \%)$. lodofurans S43 and epi-S43 were characterized by ${ }^{1} \mathrm{H}$ and ${ }^{13} \mathrm{C}$ NMR spectroscopy using pure fractions that were obtained of each diastereomer. A 61:39 mixture of diastereomers was used to obtain HRMS data, but the material decomposed before IR data was collected.

Major diastereomer (S43): ${ }^{1} \mathrm{H}$ NMR $\left(400 \mathrm{MHz}, \mathrm{CDCl}_{3}\right) \delta 7.30(\mathrm{~d}, J=8.3,2 \mathrm{H}), 7.16(\mathrm{~d}, J=7.8$, $2 \mathrm{H}), 4.91(\mathrm{~d}, J=3.4,1 \mathrm{H}), 4.21-4.27(\mathrm{~m}, 1 \mathrm{H}), 3.91-3.94(\mathrm{~m}, 1 \mathrm{H}), 3.34-3.55(\mathrm{~m}, 4 \mathrm{H}), 2.36(\mathrm{~s}, 3 \mathrm{H})$, $2.18-2.23(\mathrm{~m}, 1 \mathrm{H}), 1.85-1.92(\mathrm{~m}, 1 \mathrm{H}), 1.21(\mathrm{t}, J=6.4,3 \mathrm{H})$;

${ }^{13} \mathrm{C}$ NMR (100 MHz, $\left.\mathrm{CDCl}_{3}\right): \delta 137.9(\mathrm{C}), 137.29(\mathrm{C}), 129.1(\mathrm{CH}), 125.9(\mathrm{CH}), 86.8(\mathrm{CH}), 86.3$ $(\mathrm{CH}), 81.3(\mathrm{C}), 77.6(\mathrm{CH}), 65.1\left(\mathrm{CH}_{2}\right), 38.7\left(\mathrm{CH}_{2}\right), 21.1\left(\mathrm{CH}_{3}\right), 15.4\left(\mathrm{CH}_{3}\right), 9.5\left(\mathrm{CH}_{2}\right)$.

Minor diastereomer (epi-S43): ${ }^{1} \mathrm{H}$ NMR $\left(400 \mathrm{MHz} \mathrm{CDCl}_{3}\right) \delta 7.21(\mathrm{~d}, J=6.9,2 \mathrm{H}), 7.15(\mathrm{~d}, J=8.3$, $2 \mathrm{H}$ ), $5.07(\mathrm{~d}, J=4.1,1 \mathrm{H}$ ), 4.54 (quint, $J=9.7,1 \mathrm{H}$ ), $3.96(\mathrm{q}, J=8.3,1 \mathrm{H}$ ), $3.51(\mathrm{q}, J=6.5,2 \mathrm{H}$ ), $3.43(\mathrm{q}, J=6.5,2 \mathrm{H}), 2.34(\mathrm{~s}, 3 \mathrm{H}), 2.32-2.39(\mathrm{~m}, 1 \mathrm{H}), 2.06(\mathrm{dt}, J=13.7,4.3,1 \mathrm{H}), 1.21(\mathrm{t}, J=7.2$, $3 \mathrm{H})$;

${ }^{13} \mathrm{C}$ NMR (100 MHz, $\left.\mathrm{CDCl}_{3}\right) \delta 138.2(\mathrm{C}), 137.30(\mathrm{C}), 129.3(\mathrm{CH}), 125.5(\mathrm{CH}), 86.3(\mathrm{CH}), 85.7$ $(\mathrm{CH}), 81.2(\mathrm{C}), 79.4(\mathrm{CH}), 65.3\left(\mathrm{CH}_{2}\right), 36.7\left(\mathrm{CH}_{2}\right), 21.3\left(\mathrm{CH}_{3}\right), 15.6\left(\mathrm{CH}_{3}\right), 10.8\left(\mathrm{CH}_{2}\right)$; HRMS (ESI) m/z: [M + Na] ${ }^{+}$Calcd for $\mathrm{C}_{14} \mathrm{H}_{19} \mathrm{INaO}_{2} 369.0340$; Found 369.0348. 
$\overbrace{29}^{C_{\mathrm{OEt}}}$

$\left(2 R^{\star}, 4 R^{\star}, 5 R^{\star}\right)$-4-Ethoxy-2-(iodomethyl)-2-methyl-5-( $p$-tolyl)tetrahydrofuran $\left(2 R^{\star}, 4 S^{\star}, 5 S^{\star}\right)$-4-ethoxy-2-(iodomethyl)-2-methyl-5-( $p$-tolyl)tetrahydrofuran with $\mathrm{LiAlH}_{4}(0.004 \mathrm{~g}, 0.09 \mathrm{mmol})$ to provide alcohol $\mathbf{S 4 4}$ as a colorless oil $(0.014 \mathrm{~g}, 99 \%)$ :

${ }^{1} \mathrm{H}$ NMR: $\left(400 \mathrm{MHz}, \mathrm{CDCl}_{3}\right) \delta 7.25(\mathrm{~d}, J=7.5,2 \mathrm{H}), 7.15(\mathrm{~d}, J=7.5,2 \mathrm{H}), 4.78(\mathrm{t}, J=1.9,1 \mathrm{H}), 4.76$ (t, $J=1.9,1 \mathrm{H}), 4.50(\mathrm{q}, J=3.7,1 \mathrm{H}), 3.64(\mathrm{dq}, J=9.2,7.1,1 \mathrm{H}), 3.54(\mathrm{q}, J=5.9,1 \mathrm{H}), 3.44(\mathrm{dq}, J$ $=8.9,7.0,1 \mathrm{H}), 3.01(\mathrm{~d}, J=4.1,1 \mathrm{H}), 2.35(\mathrm{~s}, 3 \mathrm{H}), 2.16-2.18(\mathrm{~m}, J=3.6,2 \mathrm{H}), 1.72(\mathrm{~s}, 3 \mathrm{H}), 1.17$ (t, $J=7.2,3 \mathrm{H})$;

${ }^{13} \mathrm{C}$ NMR: (100 MHz, $\left.\mathrm{CDCl}_{3}\right) \delta 142.5(\mathrm{C}), 138.6(\mathrm{C}), 137.5(\mathrm{C}), 129.1(\mathrm{CH}), 126.9(\mathrm{CH}), 113.4$ $\left(\mathrm{CH}_{2}\right), 82.6(\mathrm{CH}), 75.6(\mathrm{CH}), 66.5\left(\mathrm{CH}_{2}\right), 39.7\left(\mathrm{CH}_{2}\right), 23.1\left(\mathrm{CH}_{3}\right), 21.3\left(\mathrm{CH}_{3}\right), 15.7\left(\mathrm{CH}_{3}\right)$.

Following the general procedure for cyclization, alcohol S44 $(0.014 \mathrm{~g}, 0.059 \mathrm{mmol})$ in $\mathrm{CH}_{3} \mathrm{CN}(6$ $\mathrm{mL})$ was mixed with NIS $(0.054 \mathrm{~g}, 0.24 \mathrm{mmol})$ and AgOTf $(0.003 \mathrm{~g}, 0.01 \mathrm{mmol})$ to provide the product as a $52: 48$ mixture of diastereomers as a colorless oil $(0.018 \mathrm{~g}, 86 \%)$. The major diastereomer (S45) was characterized by ${ }^{1} \mathrm{H}$ and ${ }^{13} \mathrm{C}$ NMR spectroscopy using pure fractions that were obtained. A 52:48 mixture of diastereomers was used to obtain ${ }^{1} \mathrm{H}$ and ${ }^{13} \mathrm{C}$ NMR spectra for the minor diastereomer (epi-S45), and to obtain IR and HRMS data.

Major diastereomer (S45): ${ }^{1} \mathrm{H}$ NMR $\left(400 \mathrm{MHz}, \mathrm{CDCl}_{3}\right) \delta 7.29(\mathrm{~d}, \mathrm{~J}=8.1,2 \mathrm{H}), 7.13(\mathrm{~d}, \mathrm{~J}=8.1$, $2 \mathrm{H}), 5.06(\mathrm{~d}, J=3.2,1 \mathrm{H}), 3.98(\mathrm{t}, J=3.6,1 \mathrm{H}), 3.45(\mathrm{~d}, J=9.9,1 \mathrm{H}), 3.34(\mathrm{~d}, J=10.9,1 \mathrm{H}), 3.19$ (quint, $J=7.2,1 \mathrm{H}$ ), 2.85 (quint, $J=7.2,1 \mathrm{H}$ ), $2.37(\mathrm{~d}, J=5.3,1 \mathrm{H}), 2.34(\mathrm{~s}, 3 \mathrm{H}), 2.16(\mathrm{br} \mathrm{d}, J=$ $13.9,1 \mathrm{H}), 1.68(\mathrm{~s}, 3 \mathrm{H}), 0.91(\mathrm{t}, J=7.3,3 \mathrm{H})$;

${ }^{13} \mathrm{C}$ NMR (100 MHz, $\left.\mathrm{CDCl}_{3}\right) \delta 137.2(\mathrm{C}), 134.4(\mathrm{C}), 128.6(\mathrm{CH}), 127.7(\mathrm{CH}), 85.5(\mathrm{CH}), 83.0(\mathrm{CH})$, $81.3(\mathrm{C}), 65.4\left(\mathrm{CH}_{2}\right), 44.4\left(\mathrm{CH}_{2}\right), 26.7\left(\mathrm{CH}_{3}\right), 21.4\left(\mathrm{CH}_{3}\right), 18.9\left(\mathrm{CH}_{2}\right), 15.2\left(\mathrm{CH}_{3}\right)$.

Minor diastereomer (epi-S45): ${ }^{1} \mathrm{H}$ NMR $\left(400 \mathrm{MHz} \mathrm{CDCl}_{3}\right) \delta 7.29(\mathrm{~d}, J=8.1,2 \mathrm{H}), 7.13(\mathrm{~d}, J=8.1$, $2 \mathrm{H}), 5.01(\mathrm{~d}, J=3.5,1 \mathrm{H}), 3.99(\mathrm{br} \mathrm{s}, 1 \mathrm{H}), 3.68(\mathrm{~d}, J=9.5,1 \mathrm{H}), 3.56(\mathrm{~d}, J=9.5,1 \mathrm{H}), 3.15-3.25$ $(\mathrm{m}, 1 \mathrm{H}), 2.80-2.90(\mathrm{~m}, 1 \mathrm{H}), 2.53(\mathrm{~d}, J=5.3,1 \mathrm{H}), 2.34(\mathrm{~s}, 3 \mathrm{H}), 2.09(\mathrm{dd}, J=13.9,5.1,1 \mathrm{H}), 1.54$ (s, 3H), $0.91(\mathrm{t}, \mathrm{J}=7.3,3 \mathrm{H})$;

${ }^{13} \mathrm{C} \mathrm{NMR}\left(100 \mathrm{MHz}, \mathrm{CDCl}_{3}\right) \delta 137.1(\mathrm{C}), 134.5(\mathrm{C}), 128.6(\mathrm{CH}), 127.6(\mathrm{CH}), 85.6(\mathrm{CH}), 82.3(\mathrm{CH})$, $81.5(\mathrm{C}), 65.5\left(\mathrm{CH}_{2}\right), 43.4\left(\mathrm{CH}_{2}\right), 27.3\left(\mathrm{CH}_{3}\right), 21.4\left(\mathrm{CH}_{3}\right), 17.8\left(\mathrm{CH}_{2}\right), 15.2\left(\mathrm{CH}_{3}\right)$;

IR (ATR) 2973, 2865, 1372, 1057, $1015 \mathrm{~cm}^{-1}$;

HRMS (ESI) m/z: [M + Na] ${ }^{+}$Calcd for $\mathrm{C}_{15} \mathrm{H}_{21} \mathrm{INaO}_{2}$ 383.0478; Found 383.0500.
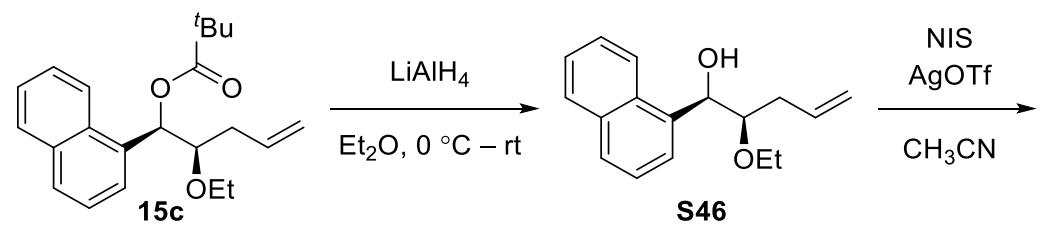

$\left(2 R^{\star}, 3 R^{\star}, 5 R^{\star}\right)-3-E$
$\left(2 R^{\star}, 3 R^{\star}, 5 S^{\star}\right)-3-e t h$
546

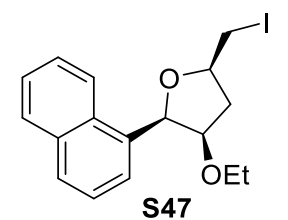

S47

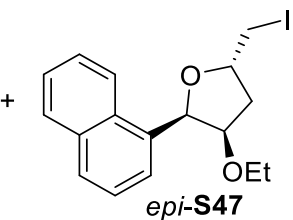

(S47) and

(epi-S47).

Following the general procedure for reduction, $15 \mathrm{c}(0.02 \mathrm{~g}, 0.006 \mathrm{mmol})$ in $\mathrm{Et}_{2} \mathrm{O}(0.6 \mathrm{~mL})$ was mixed with $\mathrm{LiAlH}_{4}(0.003 \mathrm{~g}, 0.009 \mathrm{mmol})$ to provide alcohol $\mathbf{S 4 6}$ as a colorless oil $(0.013 \mathrm{~g}, 87 \%)$ : ${ }^{1} \mathrm{H}$ NMR $\left(400 \mathrm{MHz}, \mathrm{CDCl}_{3}\right) \delta 8.11$ (dd, $\left.J=8.2,1.5,1 \mathrm{H}\right), 7.89(\mathrm{dd}, J=8.2,2.2,1 \mathrm{H}), 7.81(\mathrm{~d}, J=$ 8.2, $1 \mathrm{H}), 7.65(\mathrm{~d}, J=6.9,1 \mathrm{H}), 7.47-7.54(\mathrm{~m}, 3 \mathrm{H}), 5.85$ (ddt, $J=17.7,10.4,7.1,1 \mathrm{H}), 5.35$ (t, $J=$ 4.9, 1H), 5.09 (q, $J=1.4,1 \mathrm{H}), 5.05(\mathrm{dq}, J=7.2,1.5,1 \mathrm{H}), 3.75(\mathrm{q}, J=5.8,1 \mathrm{H}), 3.58(\mathrm{dq}, J=9.3$, 
7.1, $1 \mathrm{H}), 3.31(\mathrm{dq}, J=9.2,6.9,1 \mathrm{H}), 3.22(\mathrm{~d}, J=4.9,1 \mathrm{H}), 2.31-2.41(\mathrm{~m}, 1 \mathrm{H}), 2.24-2.31(\mathrm{~m}, 1 \mathrm{H})$, $1.13(\mathrm{t}, J=7.2,3 \mathrm{H})$;

${ }^{13} \mathrm{C}$ NMR (100 MHz, $\left.\mathrm{CDCl}_{3}\right) \delta 137.2(\mathrm{C}), 134.4(\mathrm{C}), 134.1(\mathrm{C}), 131.1(\mathrm{CH}), 129.1(\mathrm{CH}), 128.4$ $(\mathrm{CH}), 126.1(\mathrm{CH}), 125.6(\mathrm{CH}), 125.4(\mathrm{CH}), 124.9(\mathrm{CH}), 123.5(\mathrm{CH}), 117.9\left(\mathrm{CH}_{2}\right), 82.6(\mathrm{CH}), 72.3$ $(\mathrm{CH}), 66.7\left(\mathrm{CH}_{2}\right), 36.3\left(\mathrm{CH}_{2}\right), 15.6\left(\mathrm{CH}_{3}\right)$.

Following the general procedure for cyclization, alcohol $\mathbf{S 4 6}(0.013 \mathrm{~g}, 0.051 \mathrm{mmol})$ in $\mathrm{CH}_{3} \mathrm{CN}(5$ $\mathrm{mL})$ was mixed with NIS $(0.046 \mathrm{~g}, 0.21 \mathrm{mmol})$ and AgOTf $(0.003 \mathrm{~g}, 0.01 \mathrm{mmol})$ to provide the product as a $62: 38$ mixture of diastereomers and as a colorless oil $(0.016 \mathrm{~g}, 84 \%)$. $\mathbf{S 4 7}$ and epiS47 were characterized as a 77:23 mixture:

${ }^{1} \mathrm{H}$ NMR $\left(400 \mathrm{MHz}, \mathrm{CDCl}_{3}\right) \delta 7.96(\mathrm{~d}, J=8.1,1 \mathrm{H}), 7.93(\mathrm{~d}, J=8.1,0.3 \mathrm{H}), 7.88(\mathrm{~d}, J=7.5,1.3 \mathrm{H})$, 7.78-7.83 (m, 2.6H), 7.46-7.54 (m, 3.9H), $5.90(\mathrm{~d}, J=2.9,1 \mathrm{H}), 5.68(\mathrm{~d}, J=3.5,0.3 \mathrm{H}), 4.49-4.56$ $(\mathrm{m}, 1 \mathrm{H}), 4.42-4.46(\mathrm{~m}, 0.3 \mathrm{H}), 4.40(\mathrm{t}, J=3.7,1 \mathrm{H}), 4.36(\mathrm{t}, J=4.1,0.3 \mathrm{H}), 3.54(\mathrm{~d}, J=7.9,0.3 \mathrm{H})$, $3.49(\mathrm{~d}, J=5.4,1 \mathrm{H}), 2.98-3.07(\mathrm{~m}, 1.3 \mathrm{H}), 2.44-2.61(\mathrm{~m}, 2.6 \mathrm{H}), 2.23(\mathrm{ddd}, J=13.7,4.1,1.4$, $0.3 \mathrm{H}), 2.10-2.17(\mathrm{~m}, 1 \mathrm{H}), 0.62-0.66(\mathrm{~m}, 3.9 \mathrm{H})$;

Peaks in ${ }^{13} \mathrm{C}$ NMR $\left(100 \mathrm{MHz}, \mathrm{CDCl}_{3}\right)$ attributed to the major diastereomer (S47): $\delta 133.4$ (C), $133.32(\mathrm{C}), 130.8(\mathrm{C}), 129.1(\mathrm{CH}), 127.81(\mathrm{CH}), 125.90(\mathrm{CH}), 125.6(\mathrm{CH}), 125.26(\mathrm{CH}), 124.9$ $(\mathrm{CH}), 122.5(\mathrm{CH}), 82.3(\mathrm{CH}), 81.5(\mathrm{CH}), 76.9(\mathrm{CH}), 65.8\left(\mathrm{CH}_{2}\right), 41.2\left(\mathrm{CH}_{2}\right), 15.0\left(\mathrm{CH}_{3}\right), 11.9\left(\mathrm{CH}_{2}\right)$; Peaks in ${ }^{13} \mathrm{C}$ NMR $\left(100 \mathrm{MHz}, \mathrm{CDCl}_{3}\right)$ attributed to the minor diastereomer (epi-S47): $\delta 133.29$ $(\mathrm{C}), 133.1(\mathrm{C}), 130.7(\mathrm{C}), 128.9(\mathrm{CH}), 127.82(\mathrm{CH}), 125.88(\mathrm{CH}), 125.7(\mathrm{CH}), 125.23(\mathrm{CH}), 125.2$ $(\mathrm{CH}), 122.4(\mathrm{CH}), 83.2(\mathrm{CH}), 80.1(\mathrm{CH}), 78.2(\mathrm{CH}), 65.7\left(\mathrm{CH}_{2}\right), 39.4\left(\mathrm{CH}_{2}\right), 15.1\left(\mathrm{CH}_{3}\right), 10.2\left(\mathrm{CH}_{2}\right)$; IR (ATR) 2922, 2844, 1051, 1033, $906 \mathrm{~cm}^{-1}$;

HRMS (ESI) m/z: [M + Na] $]^{+}$Calcd for $\mathrm{C}_{17} \mathrm{H}_{19} \mathrm{INaO}_{2}$ 405.0322; Found 405.0324.
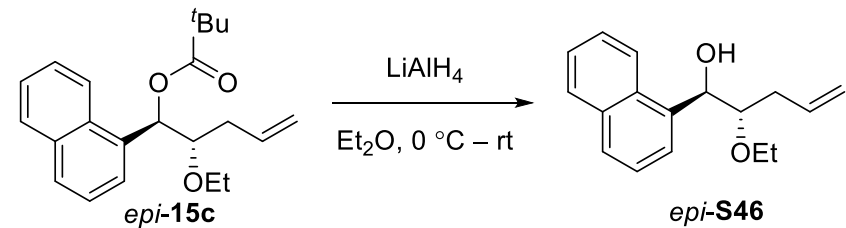

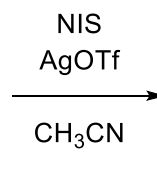

$\left(2 R^{\star}, 3 S^{\star}, 5 R^{\star}\right)-3-E t h o x y-5-($ iodomethyl)-2-(naphthalen-1-yl)tetrahydrofuran $\left(2 R^{\star}, 3 S^{\star}, 5 S^{\star}\right)$-3-ethoxy-5-(iodomethyl)-2-(naphthalen-1-yl)tetrahydrofuran

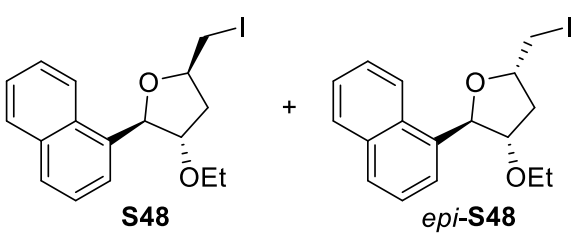

(S48) and (epi-S48).

Following the general procedure for reduction, epi-15c $(0.01 \mathrm{~g}, 0.003 \mathrm{mmol})$ in $\mathrm{Et}_{2} \mathrm{O}(0.3 \mathrm{~mL})$ was mixed with $\mathrm{LiAlH}_{4}(0.015 \mathrm{~g}, 0.44 \mathrm{mmol})$ to provide the alcohol epi-S46 as a colorless oil $(0.007 \mathrm{~g}$, 93\%):

${ }^{1} \mathrm{H}$ NMR $\left(400 \mathrm{MHz}, \mathrm{CDCl}_{3}\right) \delta 8.02(\mathrm{dd}, J=7.9,2.1,1 \mathrm{H}), 7.89(\mathrm{dd}, J=7.1,1.8,1 \mathrm{H}), 7.79-7.82(\mathrm{~m}$, 2H), 7.48-7.54 (m, 3H), 5.68-5.78 (m, 2H), 4.94-4.96 (m, 1H), $4.91(\mathrm{t}, J=1.2,1 \mathrm{H}), 3.76-3.81$ $(\mathrm{m}, 1 \mathrm{H}), 3.62-3.74(\mathrm{~m}, 2 \mathrm{H}), 3.47(\mathrm{q}, J=7.1,1 \mathrm{H}), 2.69(\mathrm{~d}, J=1.9,1 \mathrm{H}), 2.32-2.40(\mathrm{~m}, 1 \mathrm{H}), 1.21$ (t, $J=6.9,3 \mathrm{H})$;

${ }^{13} \mathrm{C}$ NMR (100 MHz, $\left.\mathrm{CDCl}_{3}\right) \delta 136.2(\mathrm{C}), 135.7(\mathrm{C}), 133.8(\mathrm{C}), 131.3(\mathrm{CH}), 129.2(\mathrm{CH}), 128.1$ $(\mathrm{CH}), 126.1(\mathrm{CH}), 125.7(\mathrm{CH}), 125.5(\mathrm{CH}), 124.3(\mathrm{CH}), 122.8(\mathrm{CH}), 116.6\left(\mathrm{CH}_{2}\right), 81.9(\mathrm{CH}), 70.9$ $(\mathrm{CH}), 65.9\left(\mathrm{CH}_{2}\right), 33.4\left(\mathrm{CH}_{2}\right), 15.7\left(\mathrm{CH}_{3}\right)$;

HRMS (ESI) m/z: [M + Na] ${ }^{+}$Calcd for $\mathrm{C}_{17} \mathrm{H}_{20} \mathrm{NaO}_{2}$ 257.2643; Found 257.2639.

Following the general procedure for cyclization, alcohol epi-S46 $(0.005 \mathrm{~g}, 0.02 \mathrm{mmol})$ in $\mathrm{CH}_{3} \mathrm{CN}$ $(2 \mathrm{~mL})$ was mixed with NIS $(0.018 \mathrm{~g}, 0.078 \mathrm{mmol})$ and AgOTf $(0.001 \mathrm{~g}, 0.004 \mathrm{mmol})$ to provide the product as a 52:48 mixture of diastereomers and as a colorless oil $(0.006 \mathrm{~g}, 86 \%)$. S48 and epi-S48 were characterized by ${ }^{1} \mathrm{H}$ and ${ }^{13} \mathrm{C}$ NMR using pure fractions that were obtained of each diastereomer. A 50:50 mixture of diastereomers was used to obtain IR and HRMS data. 
Major diastereomer (S48): ${ }^{1} \mathrm{H}$ NMR $\left(400 \mathrm{MHz}, \mathrm{CDCl}_{3}\right) \delta 8.07(\mathrm{~d}, J=8.2,1 \mathrm{H}), 7.88(\mathrm{~d}, J=8.2$, $1 \mathrm{H}), 7.78(\mathrm{~d}, J=8.2,1 \mathrm{H}), 7.44-7.57(\mathrm{~m}, 4 \mathrm{H}), 5.89(\mathrm{br} \mathrm{s}, 1 \mathrm{H}), 4.73-4.79(\mathrm{~m}, 1 \mathrm{H}), 4.11-4.14(\mathrm{~m}$, $1 \mathrm{H}), 3.46-3.68(\mathrm{~m}, 4 \mathrm{H}), 2.21-2.25(\mathrm{~m}, 2 \mathrm{H}), 1.29(\mathrm{t}, \mathrm{J}=6.9,3 \mathrm{H})$;

${ }^{13} \mathrm{C}$ NMR (100 MHz, CDCl ${ }_{3}$ ) 136.7 (C), $133.9(\mathrm{C}), 130.4(\mathrm{C}), 128.99(\mathrm{CH}), 128.1(\mathrm{CH}), 126.24$ $(\mathrm{CH}), 125.8(\mathrm{CH}), 125.53(\mathrm{CH}), 123.4(\mathrm{CH}), 122.3(\mathrm{CH}), 85.51(\mathrm{CH}), 84.9(\mathrm{CH}), 80.4(\mathrm{CH}), 65.1$ $\left(\mathrm{CH}_{2}\right), 35.0\left(\mathrm{CH}_{2}\right), 15.67\left(\mathrm{CH}_{3}\right), 10.8\left(\mathrm{CH}_{2}\right)$.

Minor diastereomer (epi-S48): ${ }^{1} \mathrm{H}$ NMR $\left(400 \mathrm{MHz} \mathrm{CDCl}_{3}\right) \delta 8.11(\mathrm{~d}, J=8.6,1 \mathrm{H}), 7.89(\mathrm{~d}, J=8.6$, $1 \mathrm{H}), 7.79(\mathrm{~m}, J=8.3,3.8,2 \mathrm{H}), 7.46-7.56(\mathrm{~m}, 3 \mathrm{H}), 5.72(\mathrm{br} \mathrm{s}, 1 \mathrm{H}), 4.33-4.39(\mathrm{~m}, 1 \mathrm{H}), 4.06(\mathrm{~d}, J$ $=5.5,1 \mathrm{H}), 3.51-3.65(\mathrm{~m}, 3 \mathrm{H}), 3.40-3.48(\mathrm{~m}, 1 \mathrm{H}), 2.30(\mathrm{dd}, J=13.2,4.9,1 \mathrm{H}), 1.75$ (dddd, $J=$ $16.1,10.3,5.8,2.9,1 \mathrm{H}), 1.29(\mathrm{t}, J=7.1,3 \mathrm{H})$;

${ }^{13} \mathrm{C}$ NMR (100 MHz, CDCl 3 ) $\delta 136.8(\mathrm{C}), 133.8(\mathrm{C}), 130.6(\mathrm{C}), 128.91(\mathrm{CH}), 128.0(\mathrm{CH}), 126.19$ $(\mathrm{CH}), 125.7(\mathrm{CH}), 125.54(\mathrm{CH}), 123.6(\mathrm{CH}), 122.9(\mathrm{CH}), 86.3(\mathrm{CH}), 85.54(\mathrm{CH}), 78.1(\mathrm{CH}), 65.2$ $\left(\mathrm{CH}_{2}\right), 38.1\left(\mathrm{CH}_{2}\right), 15.65\left(\mathrm{CH}_{3}\right), 8.6\left(\mathrm{CH}_{2}\right)$;

IR (ATR) 2922, 2844, 1051, 1033, $906 \mathrm{~cm}^{-1}$;

HRMS (ESI) m/z: [M + Na] ${ }^{+}$Calcd for $\mathrm{C}_{17} \mathrm{H}_{19} \mathrm{INaO}_{2} 405.0322$; Found 405.0323.

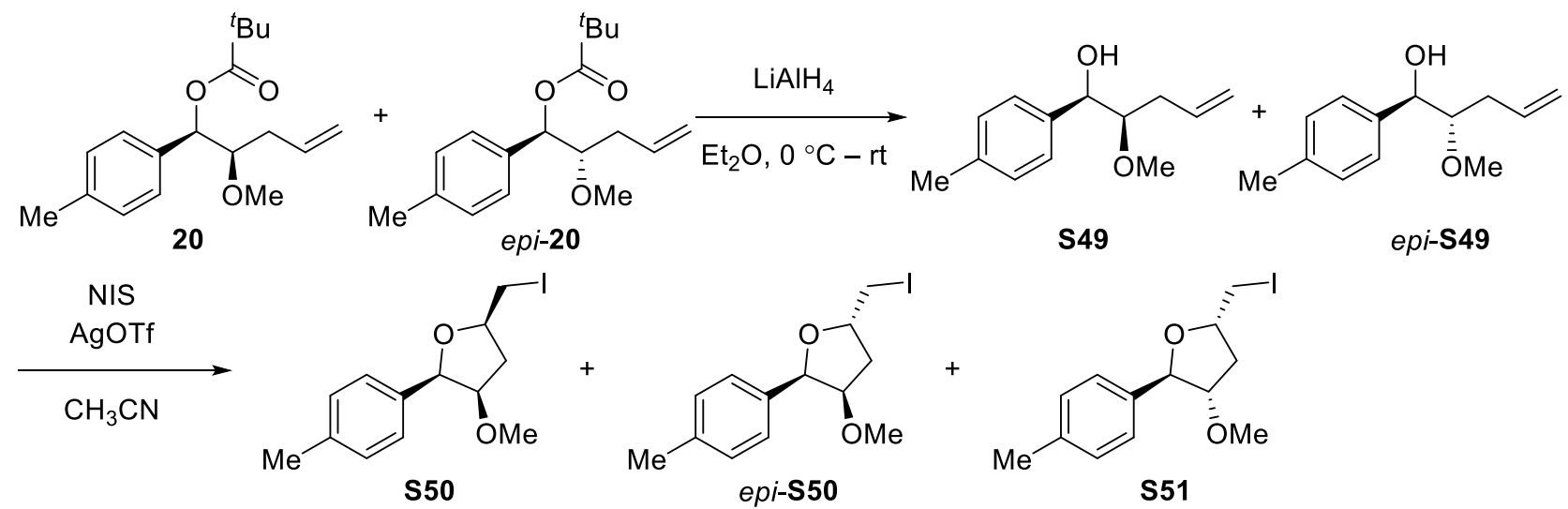

$\left(2 R^{\star}, 3 R^{\star}, 5 R^{\star}\right)-5$-(lodomethyl)-3-methoxy-2-(p-tolyl)tetrahydrofuran (S50), $\left(2 R^{\star}, 3 R^{\star}, 5 S^{\star}\right)-5$ (iodomethyl)-3-methoxy-2-( $p$-tolyl)tetrahydrofuran $\quad(e p i-S 50), \quad$ and $\quad\left(2 R^{\star}, 3 S^{\star}, 5 S^{\star}\right)-5$ (iodomethyl)-3-methoxy-2-(p-tolyl)tetrahydrofuran (S51). Following the general procedure for reduction, a 90:10 mixture of diastereomers of 20 and epi-20 (0.02 g, $0.07 \mathrm{mmol})$ in $\mathrm{Et}_{2} \mathrm{O}(0.7 \mathrm{~mL})$ was mixed with $\mathrm{LiAlH}_{4}(0.04 \mathrm{~g}, 0.1 \mathrm{mmol})$ to provide alcohols $\mathbf{S} 49$ and epi-S49 as mixture (S49:epi-S49 = 85:15) as a colorless oil $(0.015 \mathrm{~g}, 99 \%)$ :

${ }^{1} \mathrm{H}$ NMR $\left(400 \mathrm{MHz}, \mathrm{CDCl}_{3}\right) \delta 7.24(\mathrm{~d}, J=7.9,2.36 \mathrm{H}), 7.14(\mathrm{~d}, J=7.9,2.36 \mathrm{H}), 5.76-5.86(\mathrm{~m}$, $1.2 \mathrm{H}), 5.01-5.09(\mathrm{~m}, 2.36 \mathrm{H}), 4.87-4.89(\mathrm{~m}, 0.2 \mathrm{H}), 4.53(\mathrm{dd}, J=7.3,2.6,1 \mathrm{H}), 3.45(\mathrm{~s}, 3 \mathrm{H}), 3.42$ (s, 0.6H), 2.99 (br s, 1H), $2.35(\mathrm{~s}, 3.6 \mathrm{H}), 2.28-2.22(\mathrm{~m}, 1.16 \mathrm{H}), 2.05-2.12(\mathrm{~m}, 1.16 \mathrm{H})$;

Peaks in ${ }^{13} \mathrm{C}$ NMR (100 MHz, $\mathrm{CDCl}_{3}$ ) attributed to the major diastereomer (S49): $\delta 137.8(\mathrm{C})$, $137.7(\mathrm{C}), 134.1(\mathrm{CH}), 129.2(\mathrm{CH}), 127.2(\mathrm{CH}), 117.7\left(\mathrm{CH}_{2}\right), 85.1(\mathrm{CH}), 75.5(\mathrm{CH}), 58.3\left(\mathrm{CH}_{3}\right)$, $34.1\left(\mathrm{CH}_{2}\right), 21.31\left(\mathrm{CH}_{3}\right)$;

Peaks in ${ }^{13} \mathrm{C}$ NMR $\left(100 \mathrm{MHz}, \mathrm{CDCl}_{3}\right)$ attributed to the minor diastereomer (epi-S49): $\delta 137.6(\mathrm{C})$, $137.2(\mathrm{C}), 135.5(\mathrm{CH}), 129.1(\mathrm{CH}), 126.5(\mathrm{CH}), 116.9\left(\mathrm{CH}_{2}\right), 85.0(\mathrm{CH}), 73.6(\mathrm{CH}), 58.2\left(\mathrm{CH}_{3}\right)$, $33.4\left(\mathrm{CH}_{2}\right), 21.28\left(\mathrm{CH}_{3}\right)$;

HRMS (ESI) m/z: [[M + H] $\left.-\mathrm{H}_{2} \mathrm{O}\right]^{+}$Calcd for $\mathrm{C}_{13} \mathrm{H}_{17} \mathrm{O}$ 189.1274; Found 189.1287; m/z: $(\mathrm{M}+\mathrm{Na})^{+}$ Calcd for $\mathrm{C}_{13} \mathrm{H}_{18} \mathrm{NaO}_{2} 229.1199$; Found 229.1216.

Following the general procedure for cyclization, a 90:10 mixture of diastereomers of alcohols $\mathbf{S 4 9}$ and epi-S49 $(0.015 \mathrm{~g}, 0.073 \mathrm{mmol})$ in $\mathrm{CH}_{3} \mathrm{CN}(7 \mathrm{~mL})$ was mixed with NIS $(0.065 \mathrm{~g}, 0.29 \mathrm{mmol})$ and AgOTf $(0.005 \mathrm{~g}, 0.02 \mathrm{mmol})$ to provide iodofurans S50 and $\mathbf{S 5 1}$ as a mixture (S50:S51 = 
71:29), where S50 was formed as a 64:36 mixture of diastereomers and S51 was formed as a single diastereomer. lodofurans S50 and S51 were isolated as a colorless oil (0.024 g, 99\%). Further purification by column chromatography provided iodofuran $\mathbf{S} 50$ and epi-S50 as a mixture of diastereomers (S50:epi-S50 $=64: 36$ ) and iodofuran S51 as a single diastereomer. Sufficient quantities of pure iodofuran S51 were not obtained to acquire ${ }^{13} \mathrm{C}$ NMR, IR, or HRMS data.

lodofurans S50 and epi-S50: ${ }^{1} \mathrm{H}$ NMR $\left(400 \mathrm{MHz}, \mathrm{CDCl}_{3}\right)$ ס 7.24-7.30 (m, 3.2H), 7.13-7.17 (m, 3.2H), $5.08(\mathrm{~d}, J=3.4,1 \mathrm{H}), 4.89(\mathrm{~d}, J=3.4,0.56 \mathrm{H}), 4.37-4.42(\mathrm{~m}, 1 \mathrm{H}), 4.28-4.32(\mathrm{~m}, 0.5 \mathrm{H}), 3.96$ (t, $J=3.1,1 \mathrm{H}), 3.92(\mathrm{t}, J=3.1,0.56 \mathrm{H}), 3.35-3.49(\mathrm{~m}, 3.7 \mathrm{H}), 2.99(\mathrm{~s}, 3 \mathrm{H}), 2.96(\mathrm{~s}, 1.4 \mathrm{H}), 2.43$ (dd, $J=13.3,6.6,1 \mathrm{H}), 2.33(\mathrm{~s}, 4.7 \mathrm{H}), 2.17(\mathrm{dd}, J=13.3,6.6,0.56 \mathrm{H}), 1.89-1.97(\mathrm{~m}, 1.5 \mathrm{H})$;

Peaks in ${ }^{13} \mathrm{C}$ NMR $\left(100 \mathrm{MHz}, \mathrm{CDCl}_{3}\right)$ attributed to the major diastereomer (S50): $\delta 137.2(\mathrm{C})$, $134.5(\mathrm{C}), 128.8(\mathrm{CH}), 127.4(\mathrm{CH}), 84.9(\mathrm{CH}), 84.1(\mathrm{CH}), 77.2(\mathrm{CH}), 57.9\left(\mathrm{CH}_{3}\right), 39.4\left(\mathrm{CH}_{2}\right), 21.3$ $\left(\mathrm{CH}_{3}\right), 11.8\left(\mathrm{CH}_{2}\right)$;

Peaks in ${ }^{13} \mathrm{C} \mathrm{NMR} \mathrm{(100} \mathrm{MHz,} \mathrm{CDCl}_{3}$ ) attributed to the minor diastereomer (epi-S50): $\delta 137.3$ (C), 134.2 (C), $128.7(\mathrm{CH}), 127.2(\mathrm{CH}), 85.9(\mathrm{CH}), 82.9(\mathrm{CH}), 78.2(\mathrm{CH}), 57.8\left(\mathrm{CH}_{3}\right), 37.7\left(\mathrm{CH}_{2}\right), 21.2$ $\left(\mathrm{CH}_{3}\right), 10.1\left(\mathrm{CH}_{2}\right)$;

IR (ATR) 2899, 1514, 1199, 1083, $1037 \mathrm{~cm}^{-1}$;

HRMS (ESI) m/z: [M + Na] ${ }^{+}$Calcd for $\mathrm{C}_{13} \mathrm{H}_{17} / \mathrm{NaO}_{2} 355.0165$; Found 355.0172.

lodofuran S51: ${ }^{1} \mathrm{H}$ NMR (600 MHz, $\left.\mathrm{CDCl}_{3}\right) \delta 7.21(\mathrm{~d}, J=7.9,2 \mathrm{H}), 7.16(\mathrm{~d}, J=7.9,2 \mathrm{H}), 5.10(\mathrm{~d}$, $J=3.2,1 \mathrm{H}), 4.56-4.52(\mathrm{~m}, 1 \mathrm{H}), 3.87(\mathrm{dt}, J=5.9,3.16,1 \mathrm{H}), 3.39-3.44(\mathrm{~m}, 2 \mathrm{H}), 3.37(\mathrm{~s}, 3 \mathrm{H}), 2.34$ (s, 3H), 2.05-2.09 (m, 2H). 


\section{Rate experiments: Hydrolysis of acetals}

A. General Procedure

A stock solution $(1 \mathrm{~mL}, 0.1 \mathrm{M})$ of acetal $(0.1 \mathrm{mmol})$ and mesitylene $(0.3 \mathrm{mmol})$ in acetone- $\mathrm{d}_{6}$ was prepared. Experiments were performed using a commercially available stock solution of $\mathrm{DCl}$ in $\mathrm{D}_{2} \mathrm{O}\left(35 \%\right.$ in $\left.\mathrm{D}_{2} \mathrm{O}, 11.65 \mathrm{M}\right)$ or a $2.52 \mathrm{M}$ solution of $\mathrm{DCl}$ in $\mathrm{D}_{2} \mathrm{O}$ that was prepared from the $35 \%$ $\mathrm{DCl}$ in $\mathrm{D}_{2} \mathrm{O}$ solution.

\section{B. Rate Data Collection}

The stock solution containing the starting acetal and mesitylene in acetone- $\mathrm{d}_{6}(0.5 \mathrm{~mL}, 0.1 \mathrm{M})$ was added to an NMR tube and a ${ }^{1} \mathrm{H}$ single-pulse NMR spectrum was acquired. The stock solution of $\mathrm{DCl}$ in $\mathrm{D}_{2} \mathrm{O}$ was then added to the NMR tube and a second ${ }^{1} \mathrm{H}$ single-pulse experiment was performed. The sample was monitored in ten-minute increments by ${ }^{1} \mathrm{H}$ NMR spectroscopy using single-pulse experiments. The sample was removed from the probe between time points to allow for the temperature of the sample to be maintained at room temperature. Rates were calculated by following the decrease in starting material concentration and plotting In [acetal] versus time (s).

\section{Kinetic Data Processing}

The following kinetic plots were used to determine the rate constants in for acetals $4 \mathbf{a}, 4 \mathbf{d}, 6,7 \mathbf{a}$, and $\mathbf{7 b}$. Three experiments were performed for each acetal and the average rate was used. Each trendline represents $k_{\mathrm{obs}}$, and the rate of the reaction was calculated by using the equation $k=$ $k_{\mathrm{obs}} /[\mathrm{DCl}]$. An average of the rates of hydrolysis of each acetal are found below.

Graph S1. The rate of hydrolysis of acetal 4a over approximately five half-lives, which resulted in a rate of $k=2.74 \times 10^{-4}$.

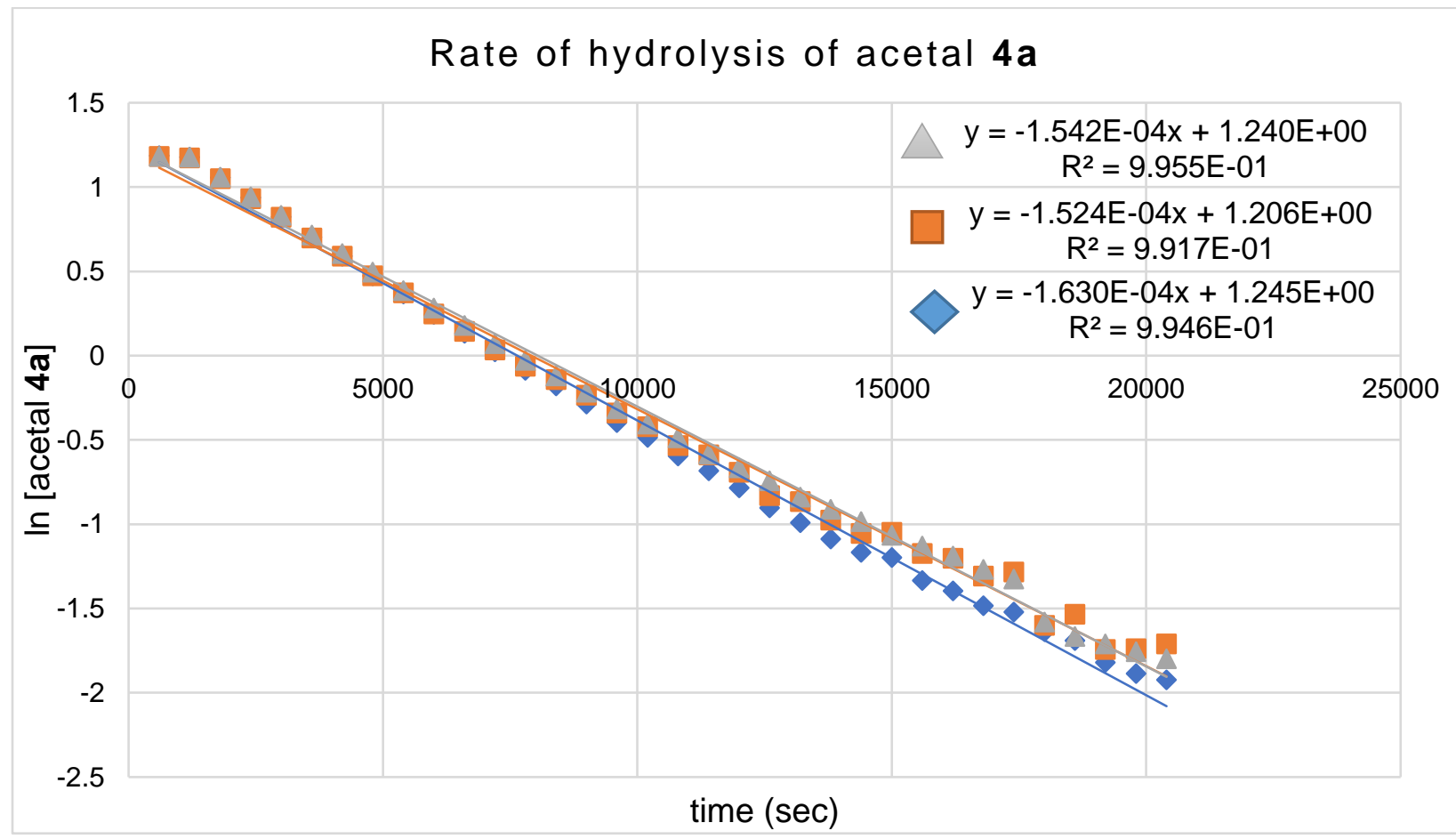


Graph S2. The rate of hydrolysis of acetal 6 over approximately four half-lives, which resulted in a rate of $k=6.89 \times 10^{-5}$.

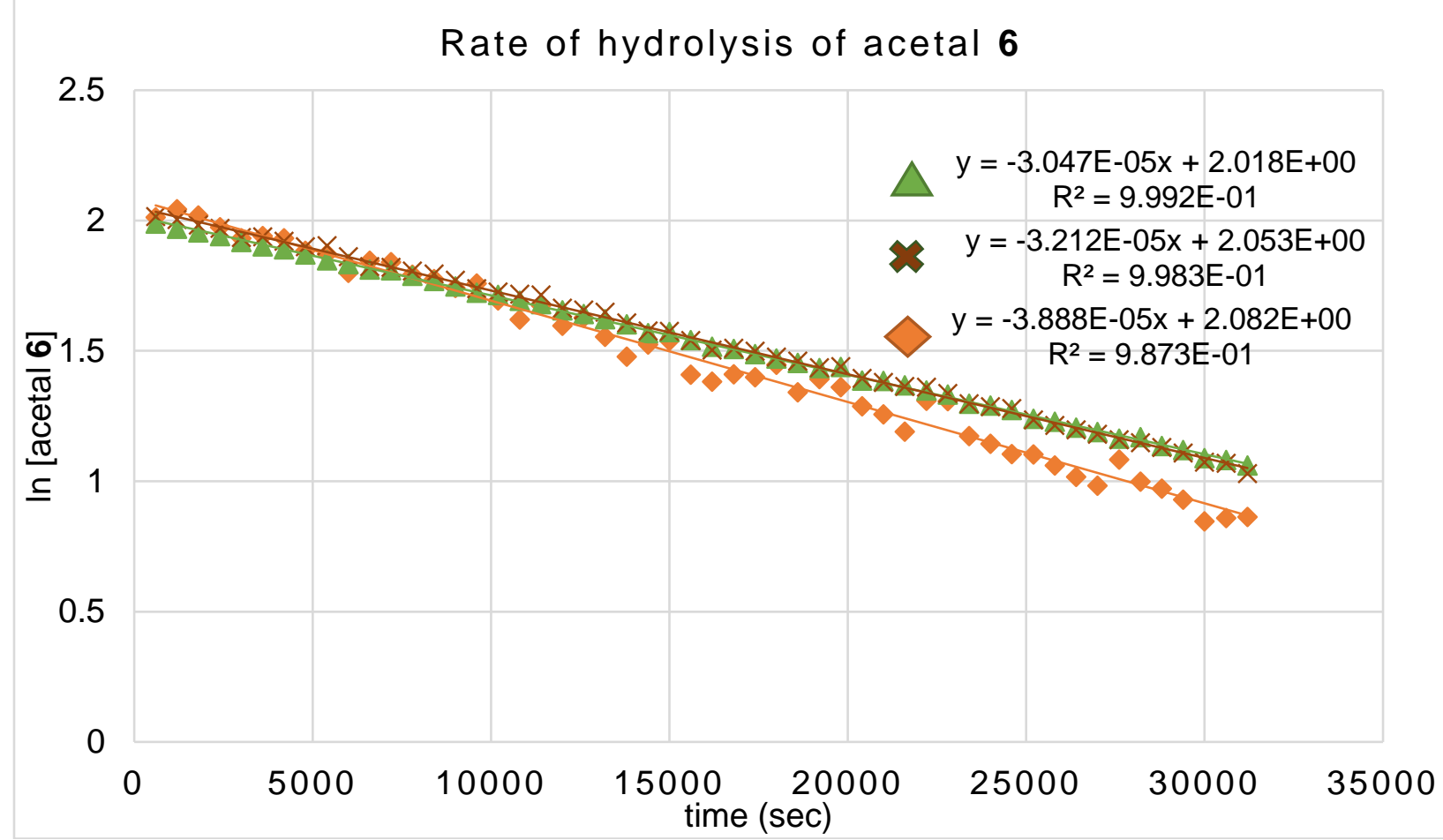

Graph S3. The rate of hydrolysis of acetal 7a over approximately five half-lives, which resulted in a rate of $k=2.05 \times 10^{-4}$.




Graph S4. The rate of hydrolysis of acetal $\mathbf{4 d}$ over approximately five half-lives, which resulted in a rate of $k=2.35 \times 10^{-4}$.

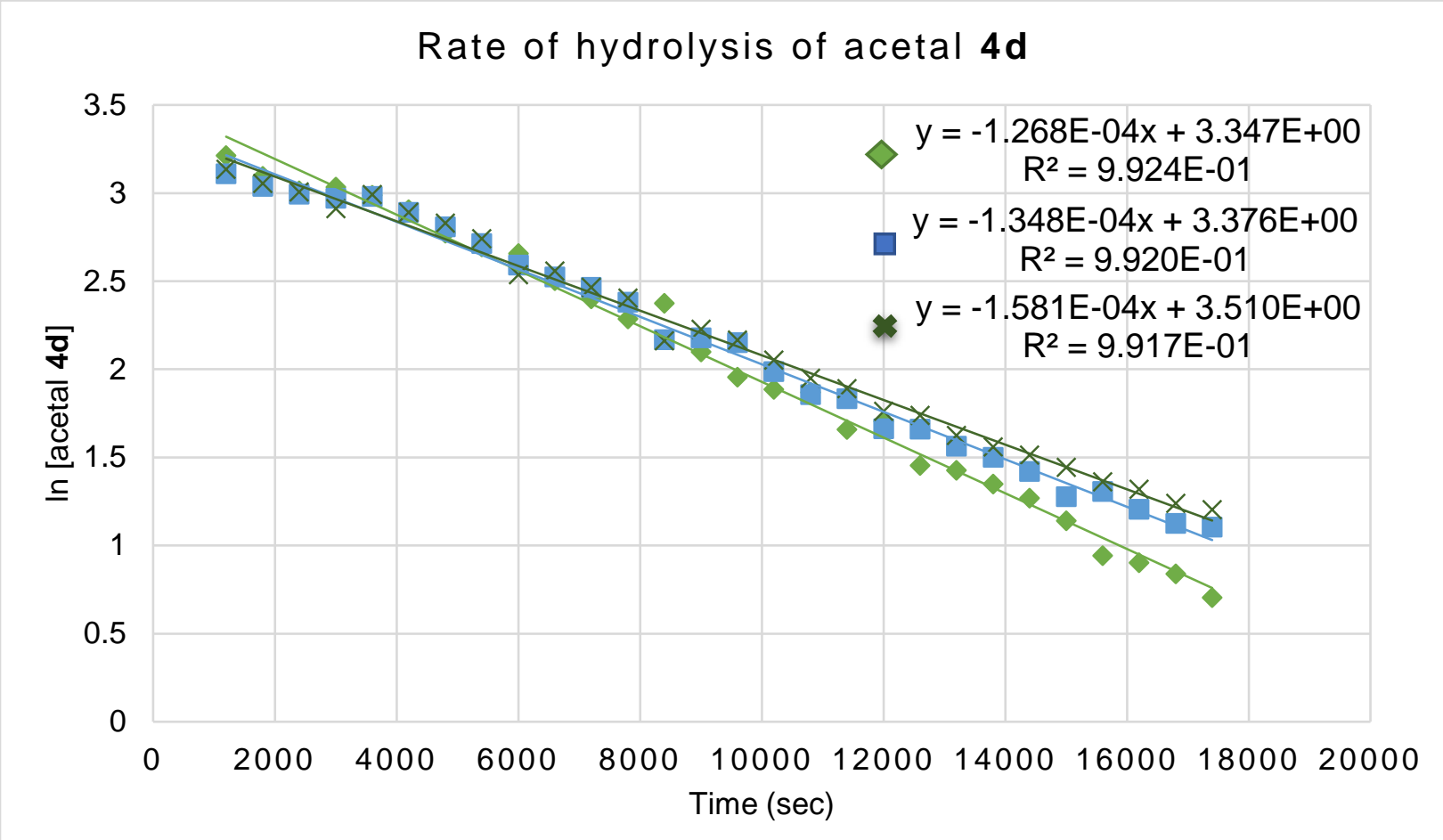

Graph S5. The rate of hydrolysis of acetal $7 \mathbf{b}$ over approximately five half-lives, which resulted in a rate of $k=3.97 \times 10^{-4}$.

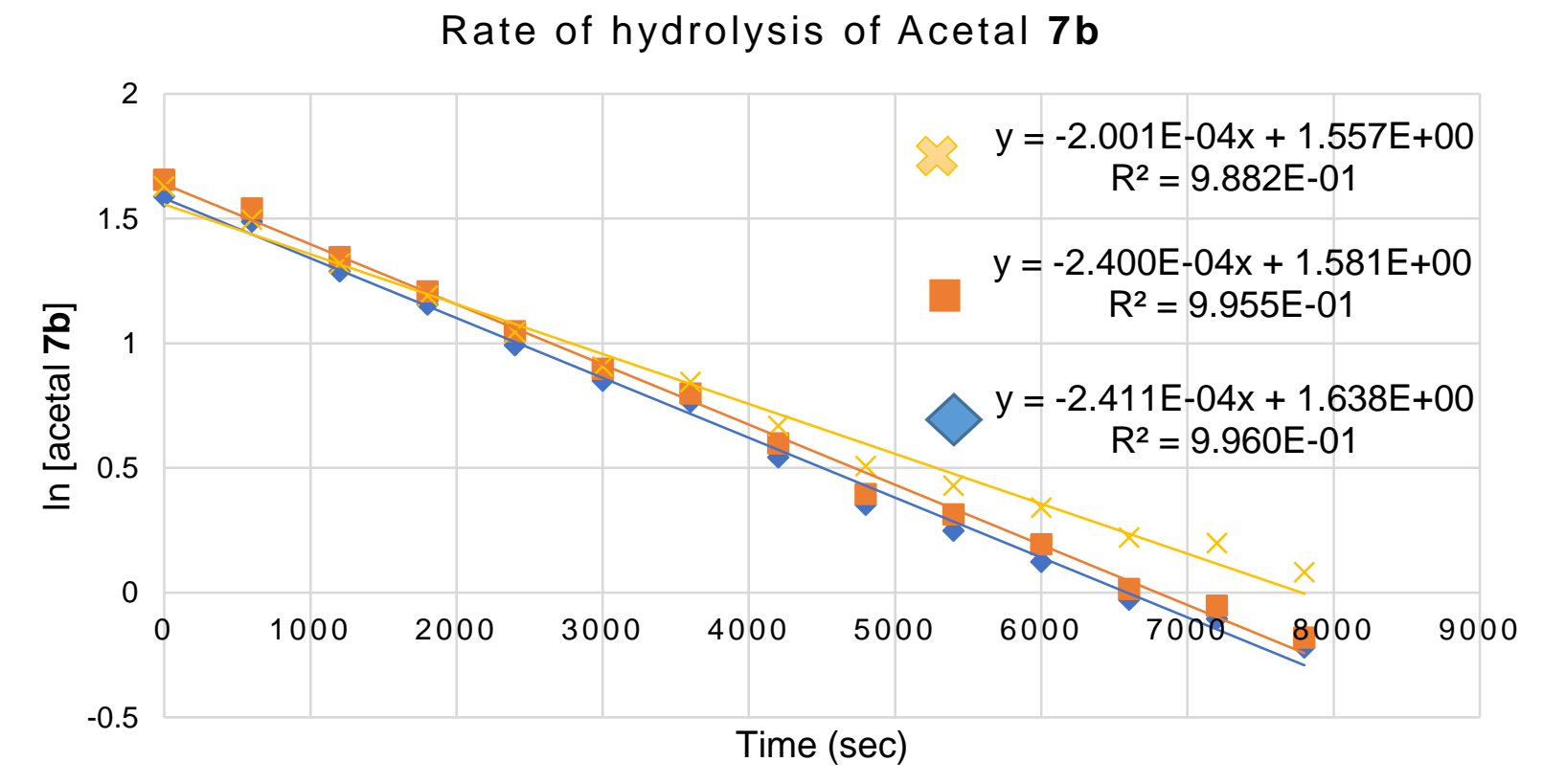




\section{Stereochemical Proofs}

\section{A. General Procedure for DPFGSE-nOe experiments}

All DPFGSE-nOe data were collected for samples prepared in degassed $\mathrm{CDCl}_{3}$ with a mixing time of 0.50 seconds. All peaks in the ${ }^{1} \mathrm{H}$ NMR spectra were assigned using ${ }^{1} \mathrm{H} /{ }^{1} \mathrm{H}$ COSY, ${ }^{1} \mathrm{H} /{ }^{13} \mathrm{C}$ HSQC, and ${ }^{1} \mathrm{H}$ NMR chemical shifts. nOe data for dioxolanes 8c, S38, 22, 26b, 29, and epi-29, were compared to the nOe data of compound $\mathbf{8 c}$, for which an X-ray crystal structure was obtained and used to confirm the stereochemical assignments. Stereochemical assignments for ethers S42, epi-S42, S43, epi-S43, S45, epi-S45, S47, epi-S47, S48, epi-S48, S50, epi-S50, and S51 were determined using the nOe data obtained.

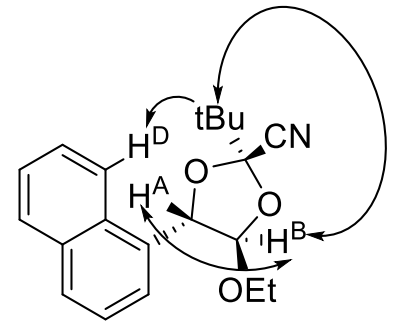

$8 \mathrm{c}$

Relevant nOe data for dioxolane $\mathbf{8 c}$

$\mathrm{H}^{\mathrm{A}}$ irradiated: $\mathrm{H}^{\mathrm{B}}(0.9 \%), \mathrm{H}^{\mathrm{B}}$ irradiated: $\mathrm{H}^{\mathrm{A}}(1.0 \%),{ }^{\mathrm{B} u} \mathrm{Bu}(3.5 \%)$,

${ }^{t}$ Bu irradiated: $\mathrm{H}^{\mathrm{B}}(0.1 \%), \mathrm{H}^{\mathrm{D}}(0.3 \%)$

Note: The nOe observed between the tert-butyl group and $\mathrm{H}^{\mathrm{B}}$ and from the tert-butyl group to $\mathrm{H}^{\mathrm{D}}$ indicate that the tert-butyl group, the naphthyl group, and $\mathrm{H}^{\mathrm{B}}$ are syn. No nOe was observed between the tert-butyl group and $\mathrm{H}^{\mathrm{A}}$, which suggests that these groups are anti, so $\mathrm{H}^{\mathrm{A}}$ and $\mathrm{H}^{\mathrm{B}}$ are also anti. This stereochemical relationship was confirmed by X-ray crystallography.

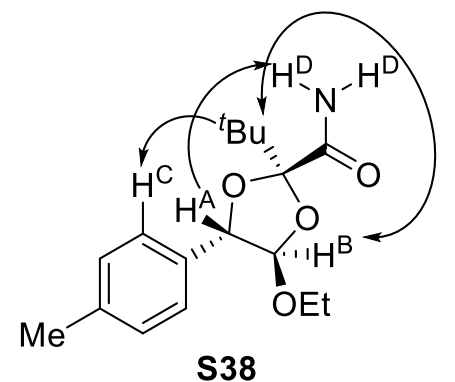

Relevant nOe data for amide S38

$H^{\mathrm{A}}$ irradiated: $\mathrm{H}^{\mathrm{D}}(39 \%)$

$\mathrm{H}^{\mathrm{B}}$ irradiated: ${ }^{\mathrm{B}} \mathrm{Bu}(3.5 \%)$

tBu irradiated: $\mathrm{H}^{\mathrm{B}}(0.4 \%), \mathrm{H}^{\mathrm{C}}(0.4 \%)$

Note: The nOe observed between the tert-butyl group and $\mathrm{H}^{\mathrm{B}}$, and from the tert-butyl group to $\mathrm{H}^{\mathrm{C}}$, indicate that $\mathrm{H}^{\mathrm{B}}$, the tert-butyl group, and the tolyl group are syn. The nOe observed from $\mathrm{H}^{\mathrm{A}}$ to $\mathrm{H}^{\mathrm{D}}$ indicate that $\mathrm{H}^{\mathrm{A}}$ and the amide are syn. These data suggest that the stereochemical relationship between $\mathrm{H}^{\mathrm{A}}$ and $\mathrm{H}^{\mathrm{B}}$ are anti. 


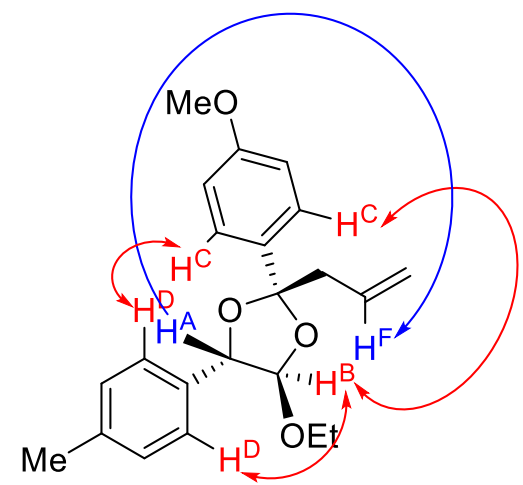

26b

Relevant nOe data for dioxolane 26b

$\mathrm{H}^{\mathrm{A}}$ irradiated: $\mathrm{H}^{\mathrm{F}}(0.8 \%)$

$H^{B}$ irradiated: $H^{C}(0.6 \%), H^{D}(1.4 \%)$

$\mathrm{H}^{\mathrm{C}}$ irradiated: $\mathrm{H}^{\mathrm{B}}(0.2 \%), \mathrm{H}^{\mathrm{D}}(0.5 \%)$

$\mathrm{H}^{\mathrm{D}}$ irradiated: $\mathrm{H}^{\mathrm{B}}(0.8 \%), \mathrm{H}^{\mathrm{C}}(6.4 \%)$

Note: The nOe observed from $\mathrm{H}^{\mathrm{A}}$ to $\mathrm{H}^{\mathrm{F}}$ indicate that $\mathrm{H}^{\mathrm{A}}$ and the allyl group are syn. The nOe observed between $\mathrm{H}^{\mathrm{B}}, \mathrm{H}^{\mathrm{C}}$, and $\mathrm{H}^{\mathrm{D}}$ indicate that $\mathrm{H}^{\mathrm{B}}$, the tolyl group, and the $p$-methoxy phenyl group are syn. These data suggest that the stereochemical relationship between $\mathrm{H}^{\mathrm{A}}$ and $\mathrm{H}^{\mathrm{B}}$ are anti.



Relevant nOe data for dioxolane 16a

$\mathrm{H}^{\mathrm{A}}$ irradiated: $\mathrm{H}^{\mathrm{C}}(0.4 \%), \mathrm{H}^{\mathrm{E}}(0.2 \%)$

$\mathrm{H}^{\mathrm{B}}$ irradiated: $\mathrm{H}^{\mathrm{D}}(0.01 \%),{ }^{\mathrm{B}} \mathrm{Bu}(0.05 \%)$

$H^{\mathrm{D}}$ irradiated: $\mathrm{H}^{\mathrm{B}}(0.8 \%)$

${ }^{t} \mathrm{Bu}$ irradiated: $\mathrm{H}^{\mathrm{B}}(0.4 \%), \mathrm{H}^{\mathrm{D}}(0.6 \%)$

Note: The nOe observed from $\mathrm{H}^{A}$ to $\mathrm{H}^{\mathrm{C}}$ indicate that $\mathrm{H}^{\mathrm{A}}$ and the allyl group are syn. The nOe observed the tert-butyl group to $\mathrm{H}^{\mathrm{B}}$ and $\mathrm{H}^{\mathrm{D}}$, and from $\mathrm{H}^{\mathrm{B}}$ to the tert-butyl group, indicate that $\mathrm{H}^{\mathrm{B}}$, the tolyl group, and the tert-butyl group are syn. These data suggest that the stereochemical relationship between $\mathrm{H}^{\mathrm{A}}$ and $\mathrm{H}^{\mathrm{B}}$ are anti. 


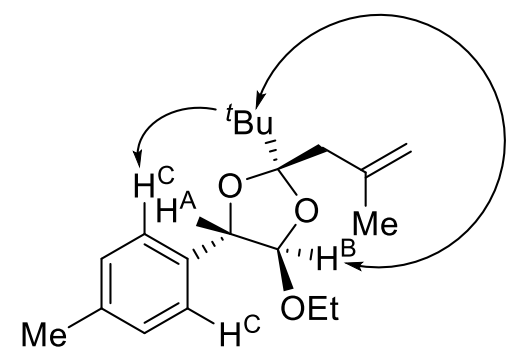

S39

Relevant nOe data for dioxolane $\mathbf{S 3 9}$

$\mathrm{H}^{\mathrm{B}}$ irradiated: ${ }^{t} \mathrm{Bu}(0.1 \%)$

${ }^{t} \mathrm{Bu}$ irradiated: $\mathrm{H}^{\mathrm{B}}(0.6 \%), \mathrm{H}^{\mathrm{C}}(0.6 \%)$

Note: The nOe observed between $\mathrm{H}^{\mathrm{B}}$ and the tert-butyl group, and from the tert-butyl group to $\mathrm{H}^{\mathrm{C}}$, indicate that $\mathrm{H}^{\mathrm{B}}$, the tolyl, and the tert-butyl groups are syn, and $\mathrm{so}^{\mathrm{A}}$, the methallyl group, and the ethoxy group are also syn. These data suggest that the stereochemical relationship between $\mathrm{H}^{\mathrm{A}}$ and $\mathrm{H}^{\mathrm{B}}$ are anti.

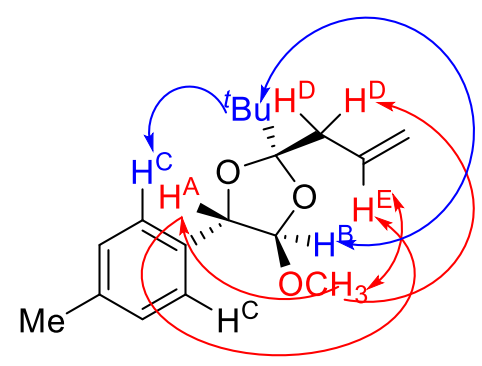

22

Relevant nOe data for dioxolane 22

$\mathrm{H}^{\mathrm{A}}$ irradiated: $\mathrm{H}^{\mathrm{E}}(1.0 \%)$

$\mathrm{H}^{\mathrm{B}}$ irradiated: ${ }^{\mathrm{B}} \mathrm{Bu}(25.7 \%)$

$\mathrm{OCH}_{3}$ irradiated: $\mathrm{H}^{\mathrm{A}}(0.4 \%), \mathrm{H}^{\mathrm{D}}(2.4 \%), \mathrm{H}^{\mathrm{E}}(0.8 \%)$

${ }^{t} \mathrm{Bu}$ irradiated: $\mathrm{H}^{\mathrm{B}}(0.2 \%), \mathrm{H}^{\mathrm{C}}(0.3 \%)$

Note: The nOe observed between the methoxy group and $\mathrm{H}^{\mathrm{A}}, \mathrm{H}^{\mathrm{D}}$, and $\mathrm{H}^{\mathrm{E}}$ indicates that $\mathrm{H}^{\mathrm{A}}$, the allyl group, and the methoxy group are syn. The nOe observed between $\mathrm{H}^{\mathrm{B}}$ and the tert-butyl group, and from the tert-butyl group to $\mathrm{H}^{\mathrm{C}}$, indicate that $\mathrm{H}^{\mathrm{B}}$, the tolyl group, and the tert-butyl group are syn. These data suggest that the stereochemical relationship between $\mathrm{H}^{\mathrm{A}}$ and $\mathrm{H}^{\mathrm{B}}$ are anti.

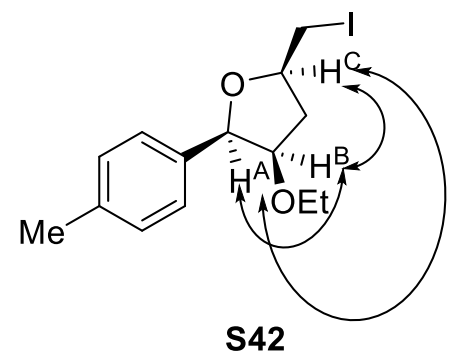

Relevant nOe data for ether $\mathbf{S 4 2}$

$\mathrm{H}^{\mathrm{A}}$ irradiated: $\mathrm{H}^{\mathrm{B}}(2.3 \%), \mathrm{H}^{\mathrm{C}}(1.1 \%)$

$\mathrm{H}^{\mathrm{B}}$ irradiated: $\mathrm{H}^{\mathrm{A}}(1.9 \%), \mathrm{H}^{\mathrm{C}}(0.5 \%)$

$\mathrm{H}^{\mathrm{C}}$ irradiated: $\mathrm{H}^{\mathrm{A}}(0.7 \%), \mathrm{H}^{\mathrm{B}}(0.3 \%)$,

Note: The nOe observed between $\mathrm{H}^{\mathrm{A}}, \mathrm{H}^{\mathrm{B}}, \mathrm{H}^{\mathrm{C}}$ indicate that $\mathrm{H}^{\mathrm{A}}, \mathrm{H}^{\mathrm{B}}$, and $\mathrm{H}^{\mathrm{C}}$ are syn, and that the tolyl, ethoxy, and iodomethyl groups are also syn to each other. 


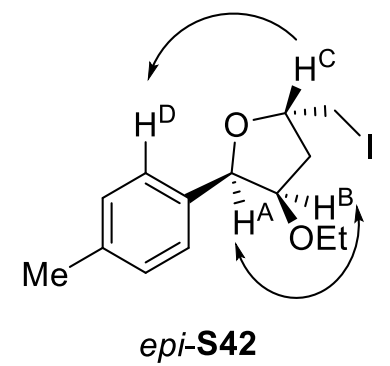

Relevant nOe data for ether epi-S42

$H^{A}$ irradiated: $H^{B}(1.9 \%)$

$H^{B}$ irradiated: $H^{A}(2.0 \%)$

$\mathrm{H}^{\mathrm{C}}$ irradiated: $\mathrm{H}^{\mathrm{D}}(0.9 \%)$

Note: There is an nOe from $\mathrm{H}^{C}$ to $\mathrm{H}^{\mathrm{D}}$, and nOe was observed from $\mathrm{H}^{\mathrm{C}}$ to $\mathrm{H}^{\mathrm{A}}$ or $\mathrm{H}^{\mathrm{B}}$, or from $\mathrm{H}^{\mathrm{A}}$ and $\mathrm{H}^{B}$ to $\mathrm{H}^{C}$. These data indicate that $\mathrm{H}^{A}$ and $\mathrm{H}^{B}$ are anti to $\mathrm{H}^{\mathrm{C}}$, and that $\mathrm{H}^{\mathrm{C}}$ is syn to the tolyl group.

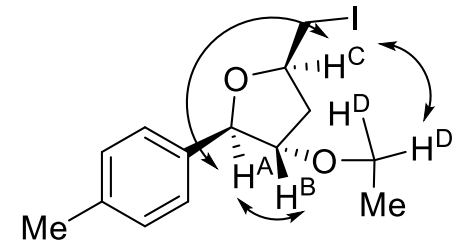

\section{S43}

Relevant nOe data for ether $\mathbf{S 4 3}$

$\mathrm{H}^{\mathrm{A}}$ irradiated: $\mathrm{H}^{\mathrm{B}}(0.4 \%), \mathrm{H}^{\mathrm{C}}(0.4 \%)$

$\mathrm{H}^{\mathrm{B}}$ irradiated: $\mathrm{H}^{\mathrm{A}}(0.4 \%)$

$\mathrm{H}^{\mathrm{C}}$ irradiated: $\mathrm{H}^{\mathrm{A}}(0.4 \%), \mathrm{H}^{\mathrm{D}}(1.8 \%)$

Note: The nOe observed from $\mathrm{H}^{\mathrm{C}}$ to $\mathrm{H}^{\mathrm{A}}$ and $\mathrm{H}^{\mathrm{D}}$, and from $\mathrm{H}^{\mathrm{A}}$ to $\mathrm{H}^{\mathrm{C}}$ indicate that $\mathrm{H}^{\mathrm{A}}, \mathrm{H}^{\mathrm{C}}$, and the ethoxy group are syn. No nOe was observed between $\mathrm{H}^{B}$ and $\mathrm{H}^{\mathrm{C}}$, which indicates that these protons are anti, and therefore $\mathrm{H}^{\mathrm{A}}$ and $\mathrm{H}^{\mathrm{B}}$ are also anti.

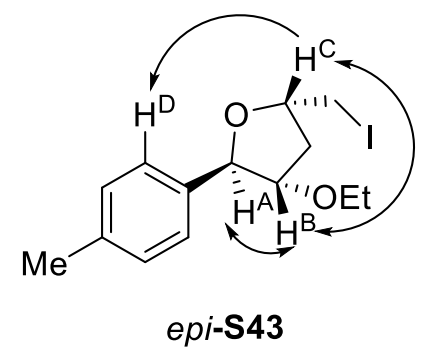

Relevant nOe data for ether epi-S43

$\mathrm{H}^{\mathrm{A}}$ irradiated: $\mathrm{H}^{\mathrm{B}}(1.9 \%)$

$\mathrm{H}^{\mathrm{B}}$ irradiated: $\mathrm{H}^{\mathrm{A}}(0.8 \%), \mathrm{H}^{\mathrm{C}}(0.5 \%)$

$\mathrm{H}^{\mathrm{C}}$ irradiated: $\mathrm{H}^{\mathrm{B}}(0.5 \%), \mathrm{H}^{\mathrm{D}}(0.6 \%)$

Note: The nOe observed between $\mathrm{H}^{\mathrm{B}}$ and $\mathrm{H}^{\mathrm{C}}$, and from $\mathrm{H}^{\mathrm{C}}$ to $\mathrm{H}^{\mathrm{D}}$ indicate that $\mathrm{H}^{\mathrm{B}}, \mathrm{H}^{\mathrm{C}}$, and the tolyl groups are syn. No nOe was observed between $\mathrm{H}^{\mathrm{A}}$ and $\mathrm{H}^{\mathrm{C}}$, which indicates that $\mathrm{H}^{\mathrm{A}}$ is anti to $\mathrm{H}^{\mathrm{B}}$ and $\mathrm{H}^{\mathrm{C}}$, and that $\mathrm{H}^{\mathrm{A}}$ is syn to the ethoxy and iodomethyl groups. 


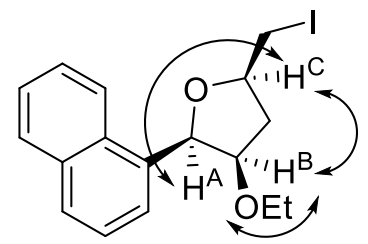

S47

Relevant nOe data for ether $\mathbf{S 4 7}$

$\mathrm{H}^{\mathrm{A}}$ irradiated: $\mathrm{H}^{\mathrm{B}}(2.4 \%), \mathrm{H}^{\mathrm{C}}(1.1 \%)$

$\mathrm{H}^{\mathrm{B}}$ irradiated: $\mathrm{H}^{\mathrm{A}}(2.0 \%), \mathrm{H}^{\mathrm{C}}(0.3 \%)$

$\mathrm{H}^{\mathrm{C}}$ irradiated: $\mathrm{H}^{\mathrm{A}}(0.7 \%), \mathrm{H}^{\mathrm{B}}(0.4 \%)$

Note: The nOe observed between $\mathrm{H}^{\mathrm{A}}, \mathrm{H}^{\mathrm{B}}$ and $\mathrm{H}^{\mathrm{C}}$ indicate that these protons are syn, and so the naphthyl, ethoxy, and iodomethyl groups are also syn.

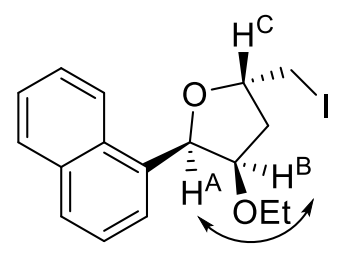

epi-S47

Relevant nOe data for ether epi-S47

$\mathrm{H}^{\mathrm{A}}$ irradiated: $\mathrm{H}^{\mathrm{B}}(2.1 \%)$

$H^{\mathrm{B}}$ irradiated: $\mathrm{H}^{\mathrm{A}}(2.3 \%)$

Note: There was no nOe observed from $\mathrm{H}^{C}$ to $\mathrm{H}^{A}$ or $\mathrm{H}^{\mathrm{B}}$, or from $\mathrm{H}^{\mathrm{A}}$ and $\mathrm{H}^{\mathrm{B}}$ to $\mathrm{H}^{\mathrm{C}}$. These data suggest that $\mathrm{H}^{\mathrm{A}}$ or $\mathrm{H}^{\mathrm{B}}$ are anti to $\mathrm{H}^{\mathrm{C}}$.

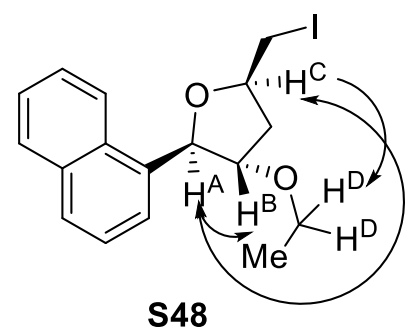

Relevant nOe data for ether $\mathbf{S 4 8}$

$\mathrm{H}^{\mathrm{A}}$ irradiated: $\mathrm{H}^{\mathrm{B}}(0.9 \%), \mathrm{H}^{\mathrm{C}}(1.1 \%)$

$\mathrm{H}^{\mathrm{B}}$ irradiated: $\mathrm{H}^{\mathrm{A}}(0.9 \%)$

$H^{C}$ irradiated: $H^{A}(1.1 \%), H^{D}(1.9 \%)$

Note: The nOe observed between $\mathrm{H}^{\mathrm{A}}$ and $\mathrm{H}^{\mathrm{C}}$ indicate that these protons are syn. The nOe from $\mathrm{H}^{\mathrm{C}}$ to $\mathrm{H}^{\mathrm{D}}$ indicate that $\mathrm{H}^{\mathrm{C}}$ is syn to the ethoxy group, and therefore $\mathrm{H}^{\mathrm{A}}, \mathrm{H}^{\mathrm{C}}$, and the ethoxy group are syn. No nOe were observed between $\mathrm{H}^{\mathrm{B}}$ and $\mathrm{H}^{\mathrm{C}}$, which indicates that these protons are anti, and so $\mathrm{H}^{\mathrm{B}}$ is also anti to $\mathrm{H}^{\mathrm{A}}$. 


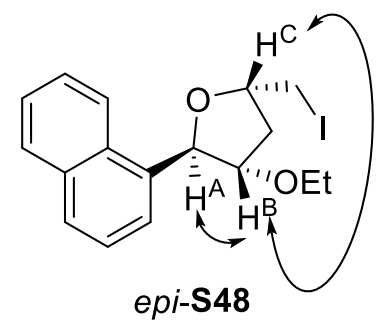

Relevant nOe data for ether epi-S48

$\mathrm{H}^{\mathrm{A}}$ irradiated: $\mathrm{H}^{\mathrm{B}}(0.9 \%)$

$\mathrm{H}^{\mathrm{B}}$ irradiated: $\mathrm{H}^{\mathrm{A}}(0.8 \%), \mathrm{H}^{\mathrm{C}}(0.5 \%)$

$\mathrm{H}^{\mathrm{C}}$ irradiated: $\mathrm{H}^{\mathrm{B}}(0.5 \%)$

Note: The nOe observed between $\mathrm{H}^{\mathrm{B}}$ and $\mathrm{H}^{\mathrm{C}}$ indicate that these protons are syn. No nOe were observed between $\mathrm{H}^{\mathrm{A}}$ and $\mathrm{H}^{\mathrm{C}}$, which indicates that these protons are anti. These data indicate that $\mathrm{H}^{\mathrm{B}}, \mathrm{H}^{\mathrm{C}}$, and the naphthyl group are syn, and so $\mathrm{H}^{\mathrm{A}}$, the ethoxy group, and the iodomethyl group are syn.

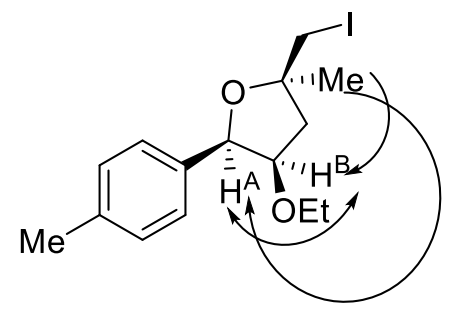

S45

Relevant nOe data for ether $\mathbf{S 4 5}$

$\mathrm{H}^{\mathrm{A}}$ irradiated: $\mathrm{H}^{\mathrm{B}}(2.1 \%)$

$\mathrm{H}^{\mathrm{B}}$ irradiated: $\mathrm{H}^{\mathrm{A}}(2.2 \%)$

Me irradiated: $\mathrm{H}^{\mathrm{A}}(0.2 \%), \mathrm{H}^{\mathrm{B}}(0.2 \%)$

Note: The nOe observed from the methyl group to $\mathrm{H}^{\mathrm{A}}$ and $\mathrm{H}^{\mathrm{B}}$, indicate that $\mathrm{H}^{\mathrm{A}}, \mathrm{H}^{\mathrm{B}}$, and the methyl group are syn, and that the tolyl, ethoxy, and iodomethyl groups are also syn.

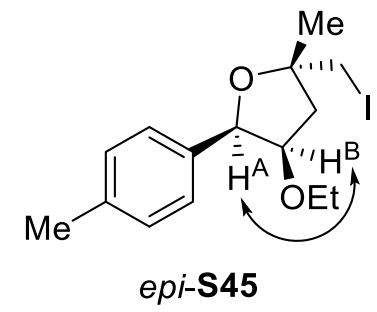

Relevant nOe data for ether epi-S45

$H^{A}$ irradiated: $H^{B}(2.0 \%)$

$\mathrm{H}^{\mathrm{B}}$ irradiated: $\mathrm{H}^{\mathrm{A}}(2.2 \%)$

Note: No nOe was observed from the methyl group to $\mathrm{H}^{\mathrm{A}}$ or $\mathrm{H}^{\mathrm{B}}$, or from $\mathrm{H}^{\mathrm{A}}$ or $\mathrm{H}^{\mathrm{B}}$ to the methyl group. These data indicate that $\mathrm{H}^{\mathrm{A}}$ and $\mathrm{H}^{\mathrm{B}}$ are anti to the methyl group, so the methyl, tolyl, and ethoxy groups are syn, and $\mathrm{H}^{\mathrm{A}}, \mathrm{H}^{\mathrm{B}}$, and the iodomethyl groups are syn. 


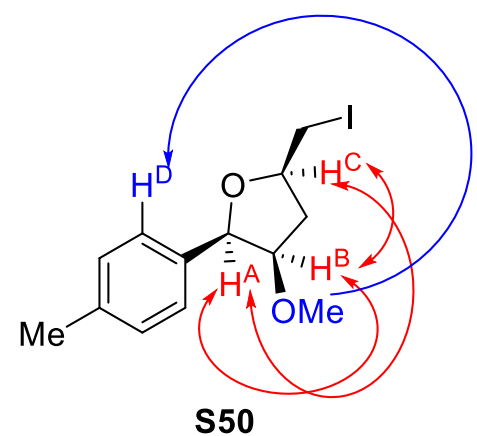

Relevant nOe data for ether S50

$\mathrm{H}^{\mathrm{A}}$ irradiated: $\mathrm{H}^{\mathrm{B}}(1.7 \%), \mathrm{H}^{\mathrm{C}}(0.8 \%)$

$\mathrm{H}^{\mathrm{B}}$ irradiated: $\mathrm{H}^{\mathrm{A}}(1.7 \%), \mathrm{H}^{\mathrm{C}}(0.4 \%)$

$\mathrm{H}^{\mathrm{C}}$ irradiated: $\mathrm{H}^{\mathrm{A}}(1.2 \%), \mathrm{H}^{\mathrm{B}}(0.7 \%)$

OMe irradiated: $\mathrm{H}^{\mathrm{D}}(1 \%)$

Note: The nOe observed between $\mathrm{H}^{\mathrm{A}}, \mathrm{H}^{\mathrm{B}}$ and $\mathrm{H}^{\mathrm{C}}$ indicate that these protons are syn. The nOe observed from the methoxy group to $\mathrm{H}^{\mathrm{D}}$ indicate that the methoxy and tolyl groups are syn.



Relevant nOe data for ether epi-S50

$H^{A}$ irradiated: $H^{B}(1.7 \%)$

$H^{B}$ irradiated: $H^{A}(1.8 \%)$

$H^{\mathrm{C}}$ irradiated: $H^{\mathrm{D}}(0.5 \%)$

OMe irradiated: $\mathrm{H}^{\mathrm{C}}(0.5 \%), \mathrm{H}^{\mathrm{D}}(0.2 \%)$,

Note: The nOe observed from $\mathrm{H}^{C}$ to $\mathrm{H}^{\mathrm{D}}$ and from the methoxy group to $\mathrm{H}^{\mathrm{C}}$ and $\mathrm{H}^{\mathrm{D}}$ indicate that $\mathrm{H}^{\mathrm{C}}$, the methoxy group, and the tolyl group are syn. No nOe was observed between $\mathrm{H}^{\mathrm{A}}$ or $\mathrm{H}^{\mathrm{B}}$ with $\mathrm{H}^{\mathrm{C}}$, which indicates that $\mathrm{H}^{\mathrm{A}}$ and $\mathrm{H}^{\mathrm{B}}$ are anti to $\mathrm{H}^{\mathrm{C}}$ and syn to the iodomethyl group.

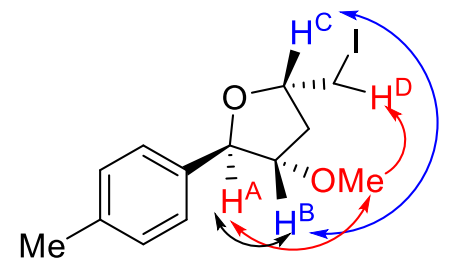

\section{S51}

Relevant nOe data for ether S51

$\mathrm{H}^{\mathrm{A}}$ irradiated: $\mathrm{H}^{\mathrm{B}}(0.7 \%)$, Me $(0.6 \%)$

$\mathrm{H}^{\mathrm{B}}$ irradiated: $\mathrm{H}^{\mathrm{A}}(0.5 \%), \mathrm{H}^{\mathrm{C}}(0.2 \%)$

$\mathrm{H}^{\mathrm{C}}$ irradiated: $\mathrm{H}^{\mathrm{B}}(0.2 \%)$

Me irradiated: $\mathrm{H}^{\mathrm{A}}(0.6 \%), \mathrm{H}^{\mathrm{D}}(1.7 \%)$

Note: The nOe observed between $\mathrm{H}^{\mathrm{B}}$ and $\mathrm{H}^{\mathrm{C}}$ indicate that these protons are syn. The nOe observed between $\mathrm{H}^{\mathrm{A}}$ and the methoxy group, and the nOe from the methoxy group to $\mathrm{H}^{\mathrm{D}}$, indicate 
that $\mathrm{H}^{\mathrm{A}}$, the methoxy group, and the iodomethyl group are syn. No nOe were observed between

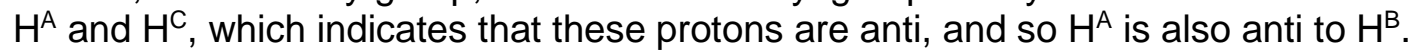


B. X-ray crystallography of $\mathbf{8 c}$.

A colorless block-like specimen of $\mathrm{C}_{20} \mathrm{H}_{23} \mathrm{NO}_{3}$, approximate dimensions $0.280 \mathrm{~mm} \times 0.450 \mathrm{~mm}$ $\times 0.560 \mathrm{~mm}$, was used for the X-ray crystallographic analysis. The X-ray intensity data were measured on a Bruker APEX-II CCD system equipped with a graphite monochromator and a Mo sealed tube $(\lambda=0.71073 \AA)$. The crystals were obtained by slow-evaporation from a 1:1 mixture of $\mathrm{Et}_{2} \mathrm{O}$ and $\mathrm{CH}_{2} \mathrm{Cl}_{2}$.

X-ray crystal structure of 8c (Deposition Number 1987988)

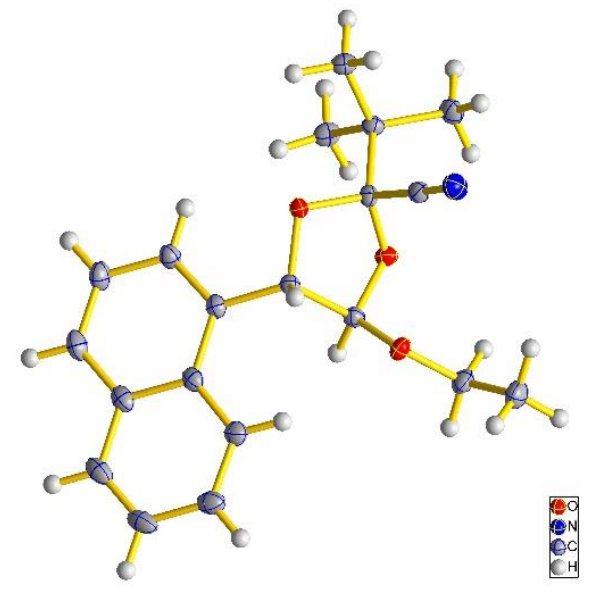

Table S1: Data collection details for $\mathbf{8 c}$.

\begin{tabular}{|c|c|c|c|c|c|c|c|c|c|c|c|c|}
\hline $\begin{array}{l}\text { Axi } \\
\text { s }\end{array}$ & $\begin{array}{l}d x / \\
m m \\
\end{array}$ & $\begin{array}{l}2 \theta / \\
0\end{array}$ & $\omega /^{\circ}$ & $\varphi /^{\circ}$ & $X I^{\circ}$ & $\begin{array}{l}\text { Wid } \\
\text { th/ }\end{array}$ & $\begin{array}{l}\text { Fra } \\
\text { mes }\end{array}$ & $\begin{array}{l}\text { Tim } \\
\text { e/s }\end{array}$ & $\begin{array}{l}\text { Wavele } \\
\text { ngth/Å }\end{array}$ & $\begin{array}{l}\text { Volta } \\
\text { ge/kV }\end{array}$ & $\begin{array}{l}\text { Curre } \\
\text { nt } / \mathrm{mA}\end{array}$ & $\begin{array}{l}\text { Temper } \\
\text { ature/KK}\end{array}$ \\
\hline $\begin{array}{l}\text { Om } \\
\text { ega }\end{array}$ & \begin{tabular}{|l|}
49. \\
995
\end{tabular} & $\begin{array}{l}- \\
28 . \\
00\end{array}$ & $\begin{array}{l}342 \\
.00\end{array}$ & $\begin{array}{l}0.0 \\
0\end{array}$ & $\begin{array}{l}54 . \\
79\end{array}$ & 0.50 & 400 & $\begin{array}{l}3.0 \\
0\end{array}$ & 0.71073 & 46 & 34.0 & 100 \\
\hline $\begin{array}{l}\text { Om } \\
\text { ega }\end{array}$ & \begin{tabular}{|l|}
49. \\
995
\end{tabular} & $\begin{array}{l}- \\
28 . \\
00\end{array}$ & $\begin{array}{l}342 \\
.00\end{array}$ & $\begin{array}{l}90 . \\
00\end{array}$ & $\begin{array}{l}54 . \\
79\end{array}$ & $\begin{array}{l}- \\
0.50\end{array}$ & 400 & $\begin{array}{l}3.0 \\
0\end{array}$ & 0.71073 & 46 & 34.0 & 100 \\
\hline $\begin{array}{l}\text { Om } \\
\text { ega }\end{array}$ & \begin{tabular}{|l|}
49. \\
995
\end{tabular} & $\begin{array}{l}- \\
28 . \\
00\end{array}$ & $\begin{array}{l}342 \\
.00\end{array}$ & $\begin{array}{l}180 \\
.00\end{array}$ & $\begin{array}{l}54 . \\
79\end{array}$ & $\begin{array}{l}- \\
0.50\end{array}$ & 400 & $\begin{array}{l}3.0 \\
0\end{array}$ & 0.71073 & 46 & 34.0 & 100 \\
\hline $\begin{array}{l}\text { Om } \\
\text { ega }\end{array}$ & \begin{tabular}{|l|}
49. \\
995
\end{tabular} & $\begin{array}{l}- \\
28 . \\
00\end{array}$ & $\begin{array}{l}342 \\
.00\end{array}$ & $\begin{array}{l}270 \\
.00\end{array}$ & $\begin{array}{l}54 . \\
79\end{array}$ & $\begin{array}{l}- \\
0.50\end{array}$ & 400 & $\begin{array}{l}3.0 \\
0\end{array}$ & 0.71073 & 46 & 34.0 & 100 \\
\hline
\end{tabular}

A total of 1600 frames were collected. The total exposure time was 1.33 hours. The frames were integrated with the Bruker SAINT software package using a narrow-frame algorithm. The integration of the data using a monoclinic unit cell yielded a total of 26542 reflections to a maximum $\theta$ angle of $28.29^{\circ}$ ( $0.75 \AA$ resolution), of which 4345 were independent (average redundancy 6.109 , completeness $\left.=99.9 \%, R_{\text {int }}=3.15 \%, R_{\text {sig }}=2.14 \%\right)$ and $3676(84.60 \%)$ were greater than $2 \sigma\left(F^{2}\right)$. The final cell constants of $\underline{a}=9.3464(5) \AA, \quad \underline{b}=10.3003(6) \AA$, $\underline{\mathrm{c}}=18.2367(10) \AA, \beta=95.2200(9)^{\circ}$, volume $=1748.38(17) \AA^{3}$, are based upon the refinement of the XYZ-centroids of 8082 reflections above $20 \sigma(l)$ with $4.485^{\circ}<2 \theta<56.08^{\circ}$. Data were corrected for absorption effects using the multi-scan method (SADABS). The ratio of minimum to 
maximum apparent transmission was 0.925 . The calculated minimum and maximum transmission coefficients (based on crystal size) are 0.6899 and 0.7457 .

The structure was solved and refined using the Bruker SHELXTL Software Package, using the space group $P 121 / n 1$, with $Z=4$ for the formula unit, $\mathrm{C}_{20} \mathrm{H}_{23} \mathrm{NO}_{3}$. The final anisotropic full-matrix least-squares refinement on $\mathrm{F}^{2}$ with 221 variables converged at $\mathrm{R} 1=4.12 \%$, for the observed data and $w R 2=11.50 \%$ for all data. The goodness-of-fit was 1.020 . The largest peak in the final difference electron density synthesis was $0.391 \mathrm{e}^{-/} \AA^{3}$ and the largest hole was $-0.216 \mathrm{e}^{-} / \AA^{3}$ with an RMS deviation of $0.046 \mathrm{e}^{-/ \AA^{3}}$. On the basis of the final model, the calculated density was $1.236 \mathrm{~g} / \mathrm{cm}^{3}$ and $\mathrm{F}(000), 696 \mathrm{e}^{-}$.

Table S2. Sample and crystal data for 8c.

\begin{tabular}{|c|c|c|}
\hline Identification code & \multicolumn{2}{|l|}{ 19kaw4h } \\
\hline Chemical formula & \multicolumn{2}{|l|}{$\mathrm{C}_{20} \mathrm{H}_{23} \mathrm{NO}_{3}$} \\
\hline Formula weight & \multicolumn{2}{|l|}{$325.40 \mathrm{~g} / \mathrm{mol}$} \\
\hline Temperature & \multicolumn{2}{|l|}{$100(2) \mathrm{K}$} \\
\hline Wavelength & \multicolumn{2}{|l|}{$0.71073 \AA$} \\
\hline Crystal size & \multicolumn{2}{|c|}{$0.280 \times 0.450 \times 0.560 \mathrm{~mm}$} \\
\hline Crystal habit & \multicolumn{2}{|l|}{ colorless block } \\
\hline Crystal system & \multicolumn{2}{|l|}{ monoclinic } \\
\hline Space group & \multicolumn{2}{|l|}{ P $121 /$ n 1} \\
\hline \multirow[t]{3}{*}{ Unit cell dimensions } & $a=9.3464(5) \AA$ & $\alpha=90^{\circ}$ \\
\hline & $\mathrm{b}=10.3003(6) \AA$ & $\beta=95.2200(9)^{\circ}$ \\
\hline & $\mathrm{C}=18.2367(10) \AA$ & $y=90^{\circ}$ \\
\hline Volume & $1748.38(17) \AA^{3}$ & \\
\hline $\mathbf{Z}$ & \multicolumn{2}{|l|}{4} \\
\hline Density (calculated) & \multicolumn{2}{|l|}{$1.236 \mathrm{~g} / \mathrm{cm}^{3}$} \\
\hline Absorption coefficient & \multicolumn{2}{|l|}{$0.083 \mathrm{~mm}^{-1}$} \\
\hline $\mathbf{F ( 0 0 0 )}$ & \multicolumn{2}{|l|}{696} \\
\hline
\end{tabular}

Table S3. Data collection and structure refinement for $\mathbf{8 c}$.

\begin{tabular}{|c|c|}
\hline Diffractometer & Bruker APEX-II CCD \\
\hline Radiation source & sealed tube, Mo \\
\hline Theta range for data collection & 2.24 to $28.29^{\circ}$ \\
\hline Index ranges & $-12<=\mathrm{h}<=12,-13<=\mathrm{k}<=13,-24<=\mathrm{k}<=24$ \\
\hline Reflections collected & 26542 \\
\hline Independent reflections & $4345[R$ (int) $=0.0315]$ \\
\hline $\begin{array}{l}\text { Coverage of independent } \\
\text { reflections }\end{array}$ & $99.9 \%$ \\
\hline Absorption correction & multi-scan \\
\hline Max. and min. transmission & 0.7457 and 0.6899 \\
\hline Structure solution technique & direct methods \\
\hline Structure solution program & SHELXT (Sheldrick 2015) \\
\hline Refinement method & Full-matrix least-squares on $\mathrm{F}^{2}$ \\
\hline Refinement program & SHELXL-2018/3 (Sheldrick, 2018) \\
\hline Function minimized & $\Sigma w\left(F_{0}^{2}-F_{c}^{2}\right)^{2}$ \\
\hline Data / restraints / parameters & $4345 / 0 / 221$ \\
\hline Goodness-of-fit on $F^{2}$ & 1.020 \\
\hline
\end{tabular}




\begin{tabular}{|l|l|l|}
\hline Final R indices & 3676 data; $\mathrm{I} 2 \mathrm{2} \sigma(\mathrm{I})$ & $\mathrm{R} 1=0.0412, \mathrm{wR} 2=0.1092$ \\
\hline Weighting scheme & all data & $\mathrm{R} 1=0.0489, \mathrm{wR} 2=0.1150$ \\
\hline Largest diff. peak and hole & $\begin{array}{l}\mathrm{w}=1 /\left[\sigma^{2}\left(\mathrm{~F}_{\mathrm{o}}{ }^{2}\right)+(0.0699 \mathrm{P})^{2}+0.3285 \mathrm{P}\right] \\
\text { where } \mathrm{P}=\left(\mathrm{F}_{\mathrm{o}}{ }^{2}+2 \mathrm{~F}_{\mathrm{c}}{ }^{2}\right) / 3\end{array}$ \\
\hline R.M.S. deviation from mean & 0.391 and $-0.216 \mathrm{e}^{-3}$ \\
\hline
\end{tabular}

Table S4. Atomic coordinates and equivalent isotropic atomic displacement parameters $\left(\AA^{2}\right)$ for $8 c$.

$\mathrm{U}(\mathrm{eq})$ is defined as one third of the trace of the orthogonalized $\mathrm{U}_{\mathrm{ij}}$ tensor.

\begin{tabular}{|l|l|l|l|l|}
\hline & $\mathbf{x} / \mathbf{a}$ & $\mathbf{y} / \mathbf{b}$ & $\mathbf{z} / \mathbf{c}$ & $\mathbf{U}(\mathbf{e q})$ \\
\hline O1 & $0.19133(8)$ & $0.58789(7)$ & $0.39720(4)$ & $0.01617(17)$ \\
\hline O2 & $0.41540(8)$ & $0.65273(7)$ & $0.43572(4)$ & $0.01708(17)$ \\
\hline O3 & $0.32980(8)$ & $0.74366(7)$ & $0.54077(4)$ & $0.01821(18)$ \\
\hline N1 & $0.20030(11)$ & $0.91359(9)$ & $0.38566(6)$ & $0.0255(2)$ \\
\hline C1 & $0.30137(11)$ & $0.67270(10)$ & $0.38036(6)$ & $0.0154(2)$ \\
\hline C2 & $0.19982(11)$ & $0.57437(10)$ & $0.47597(5)$ & $0.0154(2)$ \\
\hline C3 & $0.35030(11)$ & $0.62891(10)$ & $0.50246(6)$ & $0.0163(2)$ \\
\hline C4 & $0.34932(11)$ & $0.64529(10)$ & $0.30349(6)$ & $0.0169(2)$ \\
\hline C5 & $0.41660(13)$ & $0.50987(11)$ & $0.30343(6)$ & $0.0221(2)$ \\
\hline C6 & $0.21720(12)$ & $0.65111(11)$ & $0.24726(6)$ & $0.0204(2)$ \\
\hline C7 & $0.46000(13)$ & $0.74772(11)$ & $0.28515(7)$ & $0.0239(2)$ \\
\hline C8 & $0.24588(11)$ & $0.81054(11)$ & $0.38481(6)$ & $0.0183(2)$ \\
\hline C9 & $0.17427(11)$ & $0.43395(10)$ & $0.49535(6)$ & $0.0165(2)$ \\
\hline C10 & $0.16665(11)$ & $0.39815(10)$ & $0.57086(6)$ & $0.0177(2)$ \\
\hline C11 & $0.14155(11)$ & $0.26530(11)$ & $0.58813(6)$ & $0.0204(2)$ \\
\hline C12 & $0.12150(12)$ & $0.17311(11)$ & $0.53027(7)$ & $0.0238(2)$ \\
\hline C13 & $0.12712(13)$ & $0.21009(11)$ & $0.45877(7)$ & $0.0236(2)$ \\
\hline C14 & $0.15397(12)$ & $0.34118(10)$ & $0.44128(6)$ & $0.0198(2)$ \\
\hline C15 & $0.13470(12)$ & $0.22868(12)$ & $0.66257(7)$ & $0.0249(3)$ \\
\hline C16 & $0.15076(13)$ & $0.31835(13)$ & $0.71785(7)$ & $0.0273(3)$ \\
\hline C17 & $0.17210(12)$ & $0.44997(13)$ & $0.70108(6)$ & $0.0251(3)$ \\
\hline C18 & $0.18008(11)$ & $0.48955(11)$ & $0.62956(6)$ & $0.0206(2)$ \\
\hline C19 & $0.46204(12)$ & $0.80577(11)$ & $0.56881(6)$ & $0.0228(2)$ \\
\hline C20 & $0.42567(14)$ & $0.94260(12)$ & $0.58945(7)$ & $0.0289(3)$ \\
\hline
\end{tabular}

Table S5. Bond lengths $(\AA \AA)$ for $8 \mathrm{c}$.

\begin{tabular}{|l|l|l|l|}
\hline O1-C1 & $1.4045(12)$ & $\mathrm{O} 1-\mathrm{C} 2$ & $1.4382(12)$ \\
\hline $\mathrm{O} 2-\mathrm{C} 1$ & $1.4147(12)$ & $\mathrm{O} 2-\mathrm{C} 3$ & $1.4306(12)$ \\
\hline $\mathrm{O} 3-\mathrm{C} 3$ & $1.3950(12)$ & $\mathrm{O}-\mathrm{C} 19$ & $1.4434(13)$ \\
\hline $\mathrm{N} 1-\mathrm{C} 8$ & $1.1444(15)$ & $\mathrm{C} 1-\mathrm{C} 8$ & $1.5163(15)$ \\
\hline $\mathrm{C} 1-\mathrm{C} 4$ & $1.5363(14)$ & $\mathrm{C} 2-\mathrm{C} 9$ & $1.5130(14)$ \\
\hline $\mathrm{C} 2-\mathrm{C} 3$ & $1.5503(14)$ & $\mathrm{C} 2-\mathrm{H} 2$ & 1.0 \\
\hline $\mathrm{C} 3-\mathrm{H} 3$ & 1.0 & $\mathrm{C} 4-\mathrm{C} 5$ & $1.5301(15)$ \\
\hline $\mathrm{C} 4-\mathrm{C} 6$ & $1.5329(15)$ & $\mathrm{C} 4-\mathrm{C} 7$ & $1.5356(15)$ \\
\hline $\mathrm{C} 5-\mathrm{H} 5 \mathrm{~A}$ & 0.98 & $\mathrm{C} 5-\mathrm{H} 5 \mathrm{~B}$ & 0.98 \\
\hline $\mathrm{C} 5-\mathrm{H} 5 \mathrm{C}$ & 0.98 & $\mathrm{C} 6-\mathrm{H} 6 \mathrm{~A}$ & 0.98 \\
\hline
\end{tabular}




\begin{tabular}{|l|l|l|l|}
\hline $\mathrm{C} 6-\mathrm{H} 6 \mathrm{~B}$ & 0.98 & $\mathrm{C} 6-\mathrm{H} 6 \mathrm{C}$ & 0.98 \\
\hline $\mathrm{C} 7-\mathrm{H} 7 \mathrm{~A}$ & 0.98 & $\mathrm{C} 7-\mathrm{H} 7 \mathrm{~B}$ & 0.98 \\
\hline $\mathrm{C} 7-\mathrm{H} 7 \mathrm{C}$ & 0.98 & $\mathrm{C} 9-\mathrm{C} 14$ & $1.3737(15)$ \\
\hline $\mathrm{C} 9-\mathrm{C} 10$ & $1.4335(14)$ & $\mathrm{C} 10-\mathrm{C} 18$ & $1.4225(16)$ \\
\hline $\mathrm{C} 10-\mathrm{C} 11$ & $1.4283(15)$ & $\mathrm{C} 11-\mathrm{C} 15$ & $1.4159(16)$ \\
\hline $\mathrm{C} 11-\mathrm{C} 12$ & $1.4192(17)$ & $\mathrm{C} 12-\mathrm{C} 13$ & $1.3641(17)$ \\
\hline $\mathrm{C} 12-\mathrm{H} 12$ & 0.95 & $\mathrm{C} 13-\mathrm{C} 14$ & $1.4151(15)$ \\
\hline $\mathrm{C} 13-\mathrm{H} 13$ & 0.95 & $\mathrm{C} 14-\mathrm{H} 14$ & 0.95 \\
\hline $\mathrm{C} 15-\mathrm{C} 16$ & $1.3655(19)$ & $\mathrm{C} 15-\mathrm{H} 15$ & 0.95 \\
\hline $\mathrm{C} 16-\mathrm{C} 17$ & $1.4080(18)$ & $\mathrm{C} 16-\mathrm{H} 16$ & 0.95 \\
\hline $\mathrm{C} 17-\mathrm{C} 18$ & $1.3751(16)$ & $\mathrm{C} 17-\mathrm{H} 17$ & 0.95 \\
\hline $\mathrm{C} 18-\mathrm{H} 18$ & 0.95 & $\mathrm{C} 19-\mathrm{C} 20$ & $1.5056(17)$ \\
\hline $\mathrm{C} 19-\mathrm{H} 19 \mathrm{~A}$ & 0.99 & $\mathrm{C} 19-\mathrm{H} 19 \mathrm{~B}$ & 0.99 \\
\hline $\mathrm{C} 20-\mathrm{H} 20 \mathrm{~A}$ & 0.98 & $\mathrm{C} 20-\mathrm{H} 20 \mathrm{~B}$ & 0.98 \\
\hline $\mathrm{C} 20-\mathrm{H} 20 \mathrm{C}$ & 0.98 & & \\
\hline
\end{tabular}

Table S6. Bond angles $\left({ }^{\circ}\right)$ for $8 \mathrm{c}$.

\begin{tabular}{|l|l|l|l|}
\hline C1-O1-C2 & $107.68(7)$ & C1-O2-C3 & $106.32(7)$ \\
\hline C3-O3-C19 & $113.63(8)$ & O1-C1-O2 & $105.82(8)$ \\
\hline O1-C1-C8 & $108.05(8)$ & O2-C1-C8 & $109.70(8)$ \\
\hline O1-C1-C4 & $111.43(8)$ & O2-C1-C4 & $111.16(8)$ \\
\hline C8-C1-C4 & $110.53(8)$ & O1-C2-C9 & $109.29(8)$ \\
\hline O1-C2-C3 & $104.02(8)$ & C9-C2-C3 & $115.57(8)$ \\
\hline O1-C2-H2 & 109.2 & C9-C2-H2 & 109.2 \\
\hline C3-C2-H2 & 109.2 & O3-C3-O2 & $111.67(8)$ \\
\hline O3-C3-C2 & $107.52(8)$ & O2-C3-C2 & $103.95(8)$ \\
\hline O3-C3-H3 & 111.1 & O2-C3-H3 & 111.1 \\
\hline C2-C3-H3 & 111.1 & C5-C4-C6 & $109.96(9)$ \\
\hline C5-C4-C7 & $109.95(9)$ & C6-C4-C7 & $110.18(9)$ \\
\hline C5-C4-C1 & $108.80(9)$ & C6-C4-C1 & $108.61(8)$ \\
\hline C7-C4-C1 & $109.31(8)$ & C4-C5-H5A & 109.5 \\
\hline C4-C5-H5B & 109.5 & H5A-C5-H5B & 109.5 \\
\hline C4-C5-H5C & 109.5 & H5A-C5-H5C & 109.5 \\
\hline H5B-C5-H5C & 109.5 & C4-C6-H6A & 109.5 \\
\hline C4-C6-H6B & 109.5 & H6A-C6-H6B & 109.5 \\
\hline C4-C6-H6C & 109.5 & H6A-C6-H6C & 109.5 \\
\hline H6B-C6-H6C & 109.5 & C4-C7-H7A & 109.5 \\
\hline C4-C7-H7B & 109.5 & H7A-C7-H7B & 109.5 \\
\hline C4-C7-H7C & 109.5 & H7A-C7-H7C & 109.5 \\
\hline H7B-C7-H7C & 109.5 & N1-C8-C1 & $177.21(11)$ \\
\hline C14-C9-C10 & $119.66(10)$ & C14-C9-C2 & $120.78(9)$ \\
\hline C10-C9-C2 & $119.54(9)$ & C18-C10-C11 & $118.23(10)$ \\
\hline C18-C10-C9 & $122.95(10)$ & C11-C10-C9 & $118.81(10)$ \\
\hline C15-C11-C12 & $121.34(11)$ & C15-C11-C10 & $119.30(11)$ \\
\hline C12-C11-C10 & $119.35(10)$ & C13-C12-C11 & $120.66(11)$ \\
\hline C13-C12-H12 & 119.7 & C11-C12-H12 & 119.7 \\
\hline C12-C13-C14 & $120.32(11)$ & C12-C13-H13 & 119.8 \\
\hline C14-C13-H13 & 119.8 & C9-C14-C13 & $121.18(10)$ \\
\hline & & & \\
\hline
\end{tabular}




\begin{tabular}{|l|l|l|l|}
\hline $\mathrm{C} 9-\mathrm{C} 14-\mathrm{H} 14$ & 119.4 & $\mathrm{C} 13-\mathrm{C} 14-\mathrm{H} 14$ & 119.4 \\
\hline $\mathrm{C} 16-\mathrm{C} 15-\mathrm{C} 11$ & $121.07(11)$ & $\mathrm{C} 16-\mathrm{C} 15-\mathrm{H} 15$ & 119.5 \\
\hline $\mathrm{C} 11-\mathrm{C} 15-\mathrm{H} 15$ & 119.5 & $\mathrm{C} 15-\mathrm{C} 16-\mathrm{C} 17$ & $119.95(11)$ \\
\hline $\mathrm{C} 15-\mathrm{C} 16-\mathrm{H} 16$ & 120.0 & $\mathrm{C} 17-\mathrm{C} 16-\mathrm{H} 16$ & 120.0 \\
\hline $\mathrm{C} 18-\mathrm{C} 17-\mathrm{C} 16$ & $120.83(11)$ & $\mathrm{C} 18-\mathrm{C} 17-\mathrm{H} 17$ & 119.6 \\
\hline $\mathrm{C} 16-\mathrm{C} 17-\mathrm{H} 17$ & 119.6 & $\mathrm{C} 17-\mathrm{C} 18-\mathrm{C} 10$ & $120.59(11)$ \\
\hline $\mathrm{C} 17-\mathrm{C} 18-\mathrm{H} 18$ & 119.7 & $\mathrm{C} 10-\mathrm{C} 18-\mathrm{H} 18$ & 119.7 \\
\hline O3-C19-C20 & $107.34(9)$ & $\mathrm{O}-\mathrm{C} 19-\mathrm{H} 19 \mathrm{~A}$ & 110.2 \\
\hline $\mathrm{C} 20-\mathrm{C} 19-\mathrm{H} 19 \mathrm{~A}$ & 110.2 & $\mathrm{O}-\mathrm{C} 19-\mathrm{H} 19 \mathrm{~B}$ & 110.2 \\
\hline $\mathrm{C} 20-\mathrm{C} 19-\mathrm{H} 19 \mathrm{~B}$ & 110.2 & $\mathrm{H} 19 \mathrm{~A}-\mathrm{C} 19-\mathrm{H} 19 \mathrm{~B}$ & 108.5 \\
\hline $\mathrm{C} 19-\mathrm{C} 20-\mathrm{H} 20 \mathrm{~A}$ & 109.5 & $\mathrm{C} 19-\mathrm{C} 20-\mathrm{H} 20 \mathrm{~B}$ & 109.5 \\
\hline $\mathrm{H} 20 \mathrm{~A}-\mathrm{C} 20-\mathrm{H} 20 \mathrm{~B}$ & 109.5 & $\mathrm{C} 19-\mathrm{C} 20-\mathrm{H} 20 \mathrm{C}$ & 109.5 \\
\hline $\mathrm{H} 20 \mathrm{~A}-\mathrm{C} 20-\mathrm{H} 20 \mathrm{C}$ & 109.5 & $\mathrm{H} 20 \mathrm{~B}-\mathrm{C} 20-\mathrm{H} 20 \mathrm{C}$ & 109.5 \\
\hline
\end{tabular}

Table S7. Anisotropic atomic displacement parameters $\left(\AA^{2}\right)$ for $\mathbf{8 c}$.

The anisotropic atomic displacement factor exponent takes the form: $-2 \pi^{2}\left[h^{2} a^{* 2} U_{11}+\ldots+2 h k\right.$ $\left.a^{*} b^{*} U_{12}\right]$

\begin{tabular}{|l|l|l|l|l|l|l|}
\hline & $\mathbf{U}_{11}$ & $\mathbf{U}_{22}$ & $\mathbf{U}_{33}$ & $\mathbf{U}_{23}$ & $\mathbf{U}_{13}$ & $\mathbf{U}_{12}$ \\
\hline O1 & $0.0161(4)$ & $0.0170(4)$ & $0.0154(4)$ & $0.0012(3)$ & $0.0016(3)$ & $-0.0036(3)$ \\
\hline O2 & $0.0142(4)$ & $0.0222(4)$ & $0.0149(4)$ & $-0.0005(3)$ & $0.0014(3)$ & $-0.0003(3)$ \\
\hline O3 & $0.0179(4)$ & $0.0178(4)$ & $0.0188(4)$ & $-0.0049(3)$ & $0.0012(3)$ & $-0.0017(3)$ \\
\hline N1 & $0.0313(5)$ & $0.0196(5)$ & $0.0261(5)$ & $0.0001(4)$ & $0.0046(4)$ & $0.0016(4)$ \\
\hline C1 & $0.0139(5)$ & $0.0151(5)$ & $0.0172(5)$ & $0.0000(4)$ & $0.0012(4)$ & $-0.0012(4)$ \\
\hline C2 & $0.0158(5)$ & $0.0161(5)$ & $0.0146(5)$ & $-0.0002(4)$ & $0.0016(4)$ & $-0.0002(4)$ \\
\hline C3 & $0.0173(5)$ & $0.0168(5)$ & $0.0152(5)$ & $-0.0004(4)$ & $0.0031(4)$ & $-0.0001(4)$ \\
\hline C4 & $0.0176(5)$ & $0.0179(5)$ & $0.0153(5)$ & $-0.0009(4)$ & $0.0030(4)$ & $0.0005(4)$ \\
\hline C5 & $0.0231(5)$ & $0.0219(5)$ & $0.0213(5)$ & $-0.0028(4)$ & $0.0028(4)$ & $0.0051(4)$ \\
\hline C6 & $0.0213(5)$ & $0.0232(5)$ & $0.0164(5)$ & $-0.0005(4)$ & $0.0003(4)$ & $0.0019(4)$ \\
\hline C7 & $0.0243(6)$ & $0.0257(6)$ & $0.0228(5)$ & $-0.0005(4)$ & $0.0082(4)$ & $-0.0045(4)$ \\
\hline C8 & $0.0180(5)$ & $0.0197(5)$ & $0.0173(5)$ & $-0.0011(4)$ & $0.0029(4)$ & $-0.0021(4)$ \\
\hline C9 & $0.0139(5)$ & $0.0162(5)$ & $0.0198(5)$ & $0.0008(4)$ & $0.0032(4)$ & $0.0006(4)$ \\
\hline C10 & $0.0128(5)$ & $0.0204(5)$ & $0.0202(5)$ & $0.0028(4)$ & $0.0028(4)$ & $0.0000(4)$ \\
\hline C11 & $0.0142(5)$ & $0.0218(5)$ & $0.0255(6)$ & $0.0058(4)$ & $0.0035(4)$ & $0.0013(4)$ \\
\hline C12 & $0.0219(5)$ & $0.0164(5)$ & $0.0337(6)$ & $0.0040(4)$ & $0.0063(5)$ & $0.0009(4)$ \\
\hline C13 & $0.0257(6)$ & $0.0166(5)$ & $0.0293(6)$ & $-0.0031(4)$ & $0.0071(5)$ & $-0.0010(4)$ \\
\hline C14 & $0.0215(5)$ & $0.0180(5)$ & $0.0205(5)$ & $0.0001(4)$ & $0.0061(4)$ & $-0.0009(4)$ \\
\hline C15 & $0.0174(5)$ & $0.0290(6)$ & $0.0285(6)$ & $0.0118(5)$ & $0.0035(4)$ & $0.0013(4)$ \\
\hline C16 & $0.0194(5)$ & $0.0407(7)$ & $0.0220(6)$ & $0.0112(5)$ & $0.0033(4)$ & $0.0004(5)$ \\
\hline C17 & $0.0199(5)$ & $0.0363(7)$ & $0.0193(5)$ & $0.0000(5)$ & $0.0027(4)$ & $-0.0021(5)$ \\
\hline C18 & $0.0161(5)$ & $0.0242(5)$ & $0.0216(5)$ & $0.0007(4)$ & $0.0025(4)$ & $-0.0017(4)$ \\
\hline C19 & $0.0218(5)$ & $0.0224(6)$ & $0.0231(6)$ & $-0.0025(4)$ & $-0.0036(4)$ & $-0.0036(4)$ \\
\hline C20 & $0.0348(7)$ & $0.0215(6)$ & $0.0296(6)$ & $-0.0048(5)$ & $-0.0010(5)$ & $-0.0052(5)$ \\
\hline
\end{tabular}

Table S8. Hydrogen atomic coordinates and isotropic atomic displacement parameters $\left(\AA^{2}\right)$ for $8 c$.

\begin{tabular}{|l|l|l|l|l|}
\hline & $\mathbf{x} / \mathbf{a}$ & $\mathbf{y} / \mathbf{b}$ & $\mathbf{z} / \mathbf{c}$ & $\mathbf{U}(\mathbf{e q})$ \\
\hline H2 & 0.1244 & 0.6298 & 0.4958 & 0.019 \\
\hline
\end{tabular}




\begin{tabular}{|l|l|l|l|l|}
\hline H3 & 0.4073 & 0.5647 & 0.5342 & 0.02 \\
\hline H5A & 0.3495 & 0.4463 & 0.3209 & 0.033 \\
\hline H5B & 0.5059 & 0.5092 & 0.3360 & 0.033 \\
\hline H5C & 0.4375 & 0.4873 & 0.2533 & 0.033 \\
\hline H6A & 0.1727 & 0.7371 & 0.2489 & 0.031 \\
\hline H6B & 0.1479 & 0.5847 & 0.2591 & 0.031 \\
\hline H6C & 0.2465 & 0.6353 & 0.1978 & 0.031 \\
\hline H7A & 0.5402 & 0.7482 & 0.3237 & 0.036 \\
\hline H7B & 0.4145 & 0.8335 & 0.2823 & 0.036 \\
\hline H7C & 0.4960 & 0.7268 & 0.2377 & 0.036 \\
\hline H12 & 0.1040 & 0.0848 & 0.5415 & 0.029 \\
\hline H13 & 0.1129 & 0.1475 & 0.4205 & 0.028 \\
\hline H14 & 0.1580 & 0.3655 & 0.3913 & 0.024 \\
\hline H15 & 0.1187 & 0.1403 & 0.6742 & 0.03 \\
\hline H16 & 0.1475 & 0.2920 & 0.7676 & 0.033 \\
\hline H17 & 0.1811 & 0.5122 & 0.7397 & 0.03 \\
\hline H18 & 0.1947 & 0.5787 & 0.6192 & 0.025 \\
\hline H19A & 0.5311 & 0.8064 & 0.5307 & 0.027 \\
\hline H19B & 0.5060 & 0.7585 & 0.6125 & 0.027 \\
\hline H20A & 0.5129 & 0.9868 & 0.6103 & 0.043 \\
\hline H20B & 0.3550 & 0.9408 & 0.6260 & 0.043 \\
\hline H20C & 0.3855 & 0.9893 & 0.5455 & 0.043 \\
\hline & & & & \\
\hline
\end{tabular}


VII. HPLC traces.

Figure S1. HPLC trace of (rac)-S12.

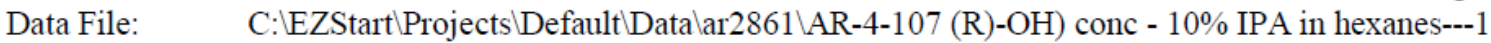
ml-min---15 min.met - 9-16-2019 4-10-11 PM.dat

Method: $\quad$ C: $\quad$ EZStart $\backslash$ Projects $\backslash$ Default $\backslash$ Method $\backslash B$ Bartolo $\backslash$ report template 220 only.met

Acquired: $\quad$ 9/16/2019 4:11:30 PM

Printed: $\quad 3 / 3 / 20205: 41: 18 \mathrm{PM}$

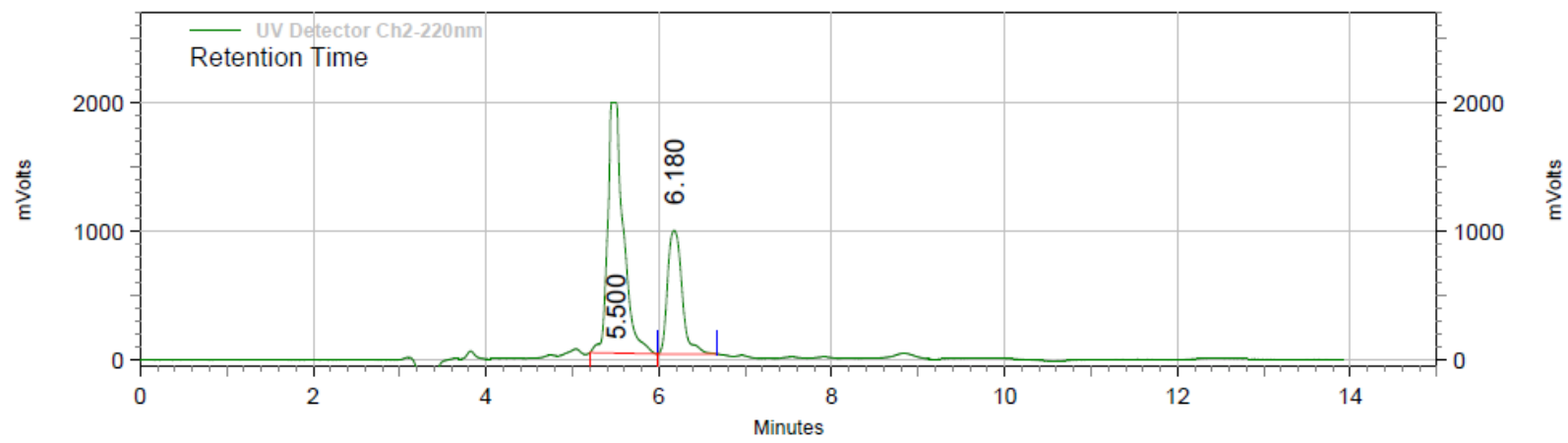

UV Detector

Ch2-220nm Results

\begin{tabular}{rrrrrr|} 
Retention Time & Area & Area \% & Height & Height \% \\
\hline 5.500 & 24545960 & 68.60 & 1946322 & 66.95 \\
6.180 & 11237004 & 31.40 & 960888 & 33.05 \\
\hline \multicolumn{2}{r|}{} & & & & \\
\hline Totals & \multirow{2}{*}{35782964} & 100.00 & 2907210 & 100.00 \\
\hline
\end{tabular}


Figure S2. HPLC trace of $(R)$-S12.

Page 1 of 1

Data File: $\quad$ C:IEZStart $\backslash$ Projects $\backslash D e f a u l t \backslash D a t a l a r 2861 \backslash A R-4-163$ alcohol - 10\% IPA in hexanes---1 ml-min---15 min.met - 9-19-2019 3-59-46 PM.dat

Method: $\quad$ C:LEZStart $\quad$ Projects $\backslash$ Default $\backslash$ Method Bartololreport template 220 only.met

Acquired: $\quad$ 9/19/2019 4:00:52 PM

Printed: $\quad 3 / 3 / 20204: 27: 14$ PM

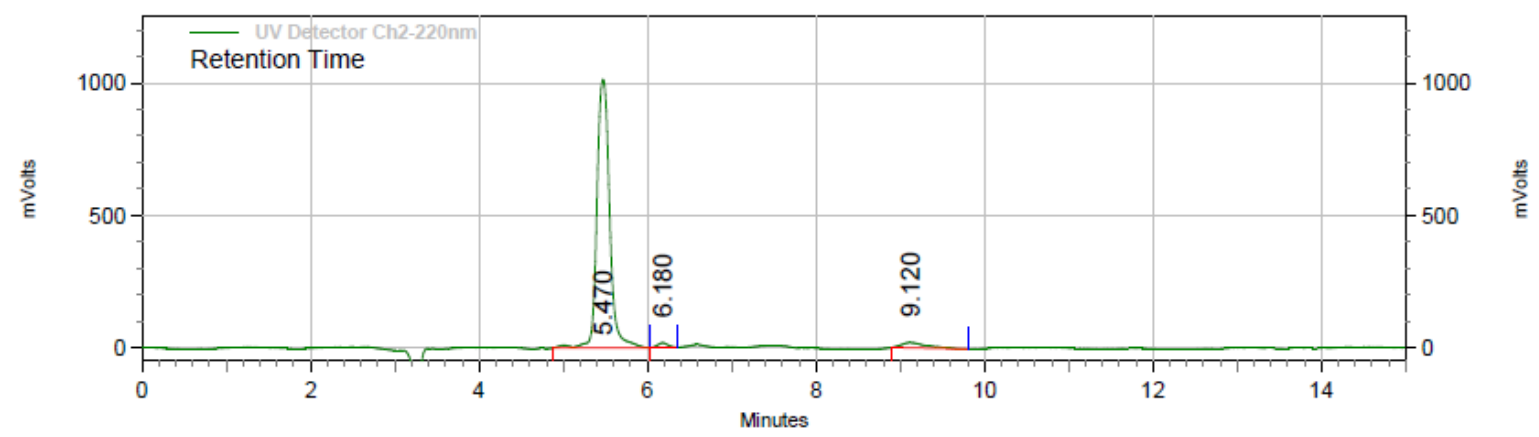

\begin{tabular}{|rrrrr|}
$\begin{array}{c}\text { UV Detector } \\
\text { Ch2-220nm Results } \\
\text { Retention Time }\end{array}$ & Area & Area \% & Height & Height \% \\
\hline 5.470 & 10603601 & 94.99 & 1014538 & 96.48 \\
6.180 & 138137 & 1.24 & 17151 & 1.63 \\
9.120 & 420763 & 3.77 & 19810 & 1.88 \\
\hline Totals & 11162501 & 100.00 & 1051499 & 100.00 \\
\hline
\end{tabular}


Figure S3. HPLC trace of (rac)-15f and epi-(rac)-15f. The peaks at retention times 3.480, 4.100, 4.510 minutes indicate the presence of two enantiomers of (rac)- $15 f$ and epi-(rac)- $15 f$. Note that the minor diastereomer of the enantiomer at 3.480 minutes could not be seperately integrated from the main peak under these conditions.

Data File: $\quad$ C:IEZStart $\quad$ Projects $\backslash$ Default Datalar2861 $\backslash A R-5-028-10 \%$ IPA in hexanes---1 ml-min---15

Page 1 of min.met - 1-10-2020 5-12-17 PM.dat

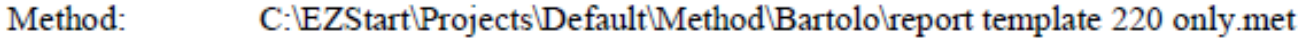

Acquired: $\quad 1 / 10 / 20205: 13: 35 \mathrm{PM}$

Printed: $\quad 3 / 3 / 20202: 36: 00$ PM

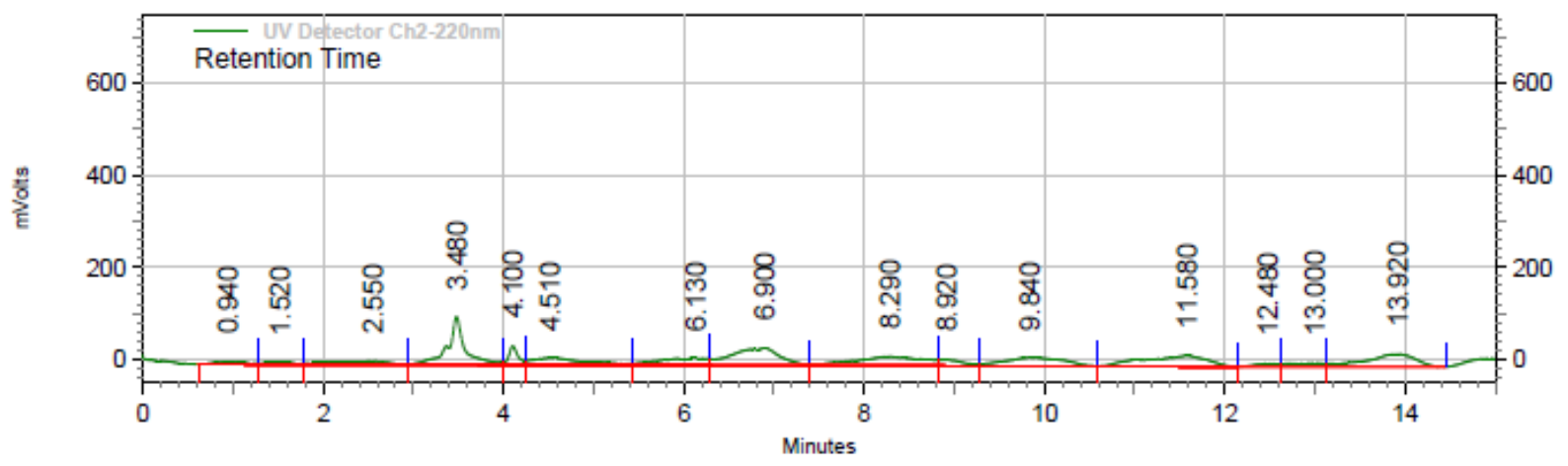

UV Detector

Ch2-220nm Results

\begin{tabular}{rrrrr} 
Retention Time & Area & Area $\%$ & Height & Height \% \\
\hline 0.940 & 141332 & 1.46 & 5588 & 1.60 \\
1.520 & 100344 & 1.04 & 4238 & 1.21 \\
2.550 & 345190 & 3.57 & 7651 & 2.19 \\
3.480 & 1394374 & 14.41 & 104242 & 29.81 \\
4.100 & 290419 & 3.00 & 40666 & 11.63 \\
4.510 & 639364 & 6.61 & 15278 & 4.37 \\
6.130 & 592539 & 6.12 & 15467 & 4.42 \\
6.900 & 1427333 & 14.75 & 38429 & 10.99 \\
8.290 & 1066772 & 11.02 & 20028 & 5.73 \\
8.920 & 250103 & 2.58 & 13589 & 3.89 \\
9.840 & 861099 & 8.90 & 18525 & 5.30 \\
11.580 & 1141290 & 11.79 & 23292 & 6.66 \\
12.480 & 122526 & 1.27 & 6869 & 1.96 \\
13.000 & 227005 & 2.35 & 8231 & 2.35 \\
13.920 & 1076784 & 11.13 & 27567 & 7.88 \\
\hline Totals & & & & 349660 \\
\hline
\end{tabular}


Figure S4. HPLC trace of $(R)-\mathbf{1 5 f}$ and epi-( $R)$-15f. The peaks at 3.500 and 4.130 indicate that only a single enantiomer of $(R)-\mathbf{1 5 f}$ and epi-(R)-15f are present.

Page 1 of 1

Data File: $\quad$ C:EZStart'ProjectsiD efault'Datalar2861 AR-4193-5 - 10\% IPA in hexanes--1 m1-min--15

min met - 1-10-2020 8-53-40 PM. dat

Method: C:EZStart'ProjectsiD efault'Method'B artolotreport template 220 only.met

Acquired: $\quad 1 / 10 / 20208: 55: 02 \mathrm{PM}$

Printed: $\quad 3 / 20202: 15: 17$ PM

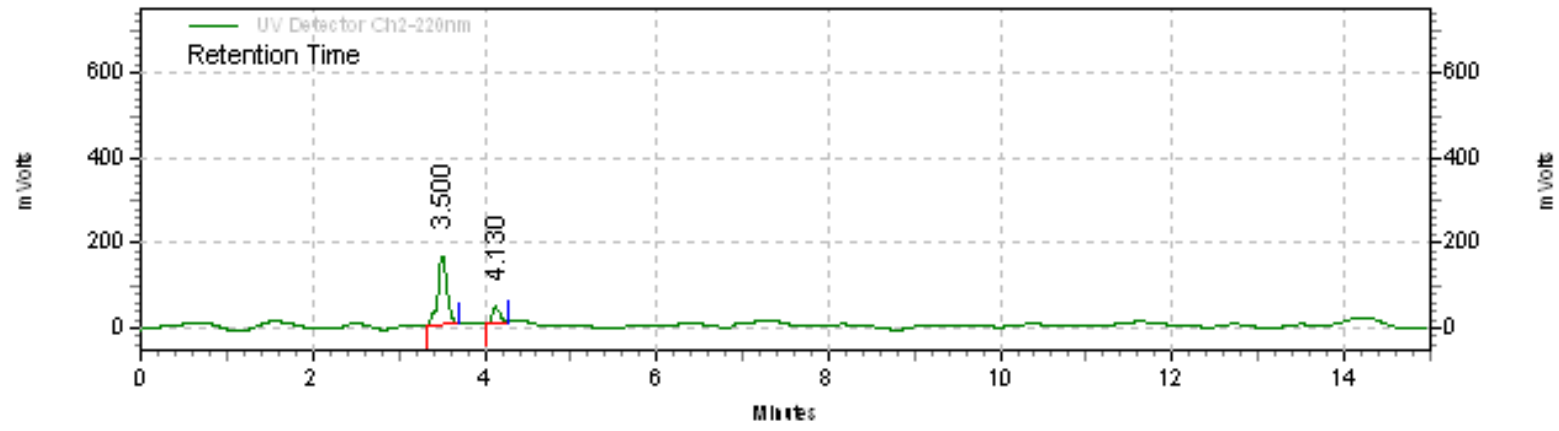

UV Detector

Ch2-220nm Results

Retention Time

3.500

4.130

Area

Area $\%$

Height

Height \%

1183078

84.94

163009

81.11

Totals

1392851

100.00

200984

100.00 


\section{Computational studies of dioxolenium ion intermediate}

Computational studies of the substitution reaction of acyclic acetals were performed on compound S53. A conformational search of S53 with molecular mechanics using Merck Molecular Force Field (MMFF) as implemented in Spartan18 produced structures S52 and S54. The conformation of S54 closely resembles the X-ray crystal structure of $\mathbf{8 c}$. This structure was further minimized using semi-empirical methods (PM3), Hartree-Fock methods (6-31G), and then density functional methods (B3LYP/6-31G). All structures were determined to be energy minima by vibrational calculations, which showed no imaginary frequencies.



Job type: Geometry optimization.

Method: RB3LYP

Job type: Frequency calculation.

Method: RB3LYP

Basis set: $6-31 G(D)$

Number of imaginary vibrations:

0

\begin{tabular}{lcccc} 
& \multicolumn{3}{c}{ Cartesian Coordinates (Angstroms) } \\
Atom & X & $Y$ & Y \\
\hline C C1 & -1.3306836 & -0.4205295 & -0.1944206 \\
C C2 & -1.2760102 & -1.6020416 & 0.7185898 \\
C C4 & -1.2703675 & -3.8221983 & 2.4126860 \\
C C3 & -2.4400755 & -2.3634374 & 0.8864813 \\
C C6 & -0.1069944 & -1.9525910 & 1.4115645 \\
C C5 & -0.1104348 & -3.0563333 & 2.2591401 \\
C C7 & -2.4341115 & -3.4774317 & 1.7253038 \\
O O2 & -0.9675244 & 0.8436506 & 0.5375409 \\
C C9 & -0.3888098 & -0.3657967 & -1.4217108 \\
O O3 & 0.7075151 & -1.1167961 & -1.3091537 \\
C C11 & 1.4842937 & -1.3397265 & -2.5296234 \\
C C12 & 2.4686180 & -2.4563674 & -2.2571145 \\
C C13 & -0.2742166 & 1.6139623 & -0.2312605
\end{tabular}




$\begin{array}{lrrr}\text { O O1 } & 0.0164938 & 1.1408349 & -1.3892846 \\ \text { C C15 } & 0.1808017 & 2.9666456 & 0.2368774 \\ \text { C C16 } & 1.2598973 & 2.7072261 & 1.3282025 \\ \text { C C17 } & -1.0278580 & 3.7106148 & 0.8550125 \\ \text { C C18 } & 0.7782584 & 3.7632583 & -0.9368338 \\ \text { H H19 } & -2.3543398 & -0.2405051 & -0.5300218 \\ \text { H H20 } & -1.2657988 & -4.6849353 & 3.0721176 \\ \text { H H21 } & -3.3521625 & -2.0885784 & 0.3618381 \\ \text { H H22 } & 0.7984415 & -1.3673686 & 1.2909965 \\ \text { H H23 } & 0.7926393 & -3.3221493 & 2.8000515 \\ \text { H H24 } & -3.3379081 & -4.0661538 & 1.8474609 \\ \text { H H25 } & -0.9061464 & -0.4472202 & -2.3818106 \\ \text { H H26 } & 1.9876189 & -0.4021640 & -2.7876701 \\ \text { H H27 } & 0.7831315 & -1.5983453 & -3.3331083 \\ \text { H H28 } & 3.1540502 & -2.1871349 & -1.4479976 \\ \text { H H29 } & 3.0608419 & -2.6439291 & -3.1584228 \\ \text { H H30 } & 1.9477039 & -3.3791565 & -1.9865698 \\ \text { H H31 } & 0.8500054 & 2.1553574 & 2.1787336 \\ \text { H H32 } & 2.1182355 & 2.1585974 & 0.9264963 \\ \text { H H33 } & 1.6171295 & 3.6775842 & 1.6862045 \\ \text { H H34 } & -1.4696913 & 3.1524550 & 1.6846974 \\ \text { H H35 } & -1.8028559 & 3.9046109 & 0.1063947 \\ \text { H H36 } & -0.6793877 & 4.6746757 & 1.2361445 \\ \text { H H37 } & 1.1057863 & 4.7381505 & -0.5650620 \\ \text { H H38 } & 1.6443717 & 3.2600429 & -1.3764616 \\ \text { H H39 } & 0.0395434 & 3.9332232 & -1.7260079 \\ \text { Point Group }=\text { C1 Order }=1 \text { Nsymop }= & 1\end{array}$

\section{References:}

Wavefunction Developers:

B.J. Deppmeier, A.J. Driessen, W.J. Hehre, T.S. Hehre, J.A. Johnson, S. Ohlinger, P.E. Klunzinger

Please cite Spartan as:

Spartan 18

Wavefunction Inc.

Irvine CA

Q-Chem 5.1, Q-Chem, Inc., Pleasanton, CA (2019)

Q-Chem Developers:

Yihan Shao, Zhengting Gan, E. Epifanovsky, A. T. B. Gilbert, M. Wormit, J. Kussmann, A. W. Lange, A. Behn, Jia Deng, Xintian Feng, D. Ghosh,

M. Goldey, P. R. Horn, L. D. Jacobson, I. Kaliman, T. Kus, A. Landau, Jie Liu,

E. I. Proynov, R. M. Richard, R. P. Steele, E. J. Sundstrom,

H. L. Woodcock III, P. M. Zimmerman, D. Zuev, B. Albrecht, E. Alguire,

S. A. Baeppler, D. Barton, Z. Benda, Y. A. Bernard, E. J. Berquist,

K. B. Bravaya, H. Burton, D. Casanova, Chun-Min Chang, Yunqing Chen, A. Chien,

K. D. Closser, M. P. Coons, S. Coriani, S. Dasgupta, A. L. Dempwolff,

M. Diedenhofen, Hainam Do, R. G. Edgar, Po-Tung Fang, S. Faraji, S. Fatehi,

Qingguo Feng, K. D. Fenk, J. Fosso-Tande, Qinghui Ge, A. Ghysels,

G. Gidofalvi, J. Gomes, J. Gonthier, A. Gunina, D. Hait,

M. W. D. Hanson-Heine, P. H. P. Harbach, A. W. Hauser, J. E. Herr,

E. G. Hohenstein, Z. C. Holden, Kerwin Hui, B. C. Huynh, T.-C. Jagau, 
Hyunjun Ji, B. Kaduk, K. Khistyaev, Jaehoon Kim, P. Klunzinger, K. Koh, D. Kosenkov, L. Koulias, T. Kowalczyk, C. M. Krauter, A. Kunitsa, Ka Un Lao, A. Laurent, K. V. Lawler, Joonho Lee, D. Lefrancois, S. Lehtola, D. S. Levine, Yi-Pei Li, You-Sheng Lin, Fenglai Liu, E. Livshits, A. Luenser, P. Manohar, E. Mansoor, S. F. Manzer, Shan-Ping Mao, Yuezhi Mao, N. Mardirossian, A. V. Marenich, T. Markovich, L. A. Martinez-Martinez, S. A. Maurer, N. J. Mayhall, S. C. McKenzie, J.-M. Mewes, A. F. Morrison, J. W. Mullinax, K. Nanda, T. S. Nguyen-Beck, R. Olivares-Amaya, J. A. Parkhill, T. M. Perrine, F. Plasser, P. Pokhilko, S. Prager, A. Prociuk, E. Ramos, D. R. Rehn, F. Rob, M. Schneider, N. Sergueev, S. M. Sharada, S. Sharma, D. W. Small, T. Stauch, T. Stein, Yu-Chuan Su, A. J. W. Thom, A. Tkatchenko, T. Tsuchimochi,

N. M. Tubman, L. Vogt, M. L. Vidal, O. Vydrov, M. A. Watson, J. Wenzel, M. de Wergifosse, T. A. Wesolowski, A. White, J. Witte, A. Yamada, Jun Yang, K. Yao, S. Yeganeh, S. R. Yost, Zhi-Qiang You, A. Zech, Igor Ying Zhang, Xing Zhang, Yan Zhao, Ying Zhu, B. R. Brooks, G. K. L. Chan, C. J. Cramer, M. S. Gordon, W. J. Hehre, A. Klamt, M. W. Schmidt, C. D. Sherrill, D. G. Truhlar, A. Aspuru-Guzik, R. Baer, A. T. Bell, N. A. Besley, Jeng-Da Chai, A. E. DePrince, III, R. A. DiStasio Jr., A. Dreuw, B. D. Dunietz, T. R. Furlani, Chao-Ping Hsu, Yousung Jung, Jing Kong, D. S. Lambrecht, WanZhen Liang, C. Ochsenfeld, V. A. Rassolov, L. V. Slipchenko, J. E. Subotnik, T. Van Voorhis, J. M. Herbert, A. I. Krylov, P. M. W. Gill, M. Head-Gordon,

Contributors to earlier versions of Q-Chem not listed above:

R. D. Adamson, B. Austin, J. Baker, G. J. O. Beran, K. Brandhorst, S. T. Brown, E. F. C. Byrd, A. K. Chakraborty, C.-L. Cheng, Siu Hung Chien, D. M. Chipman, D. L. Crittenden, H. Dachsel, R. J. Doerksen, A. D. Dutoi, L. Fusti-Molnar, W. A. Goddard III, A. Golubeva-Zadorozhnaya, S. R. Gwaltney, G. Hawkins, A. Heyden, S. Hirata, G. Kedziora, F. J. Keil, C. Kelley, Jihan Kim, R. A. King, R. Z. Khaliullin, P. P. Korambath, W. Kurlancheek, A. M. Lee, M. S. Lee, S. V. Levchenko, Ching Yeh Lin, D. Liotard, R. C. Lochan, I. Lotan, P. E. Maslen, N. Nair, D. P. O'Neill, D. Neuhauser, E. Neuscamman, C. M. Oana, R. Olson, B. Peters, R. Peverati, P. A. Pieniazek, Y. M. Rhee, J. Ritchie, M. A. Rohrdanz, E. Rosta, N. J. Russ,

H. F. Schaefer III, N. E. Schultz, N. Shenvi, A. C. Simmonett, A. Sodt,

D. Stuck, K. S. Thanthiriwatte, V. Vanovschi, Tao Wang, A. Warshel,

C. F. Williams, Q. Wu, X. Xu, W. Zhang,

Y.Shao et al., Mol.Phys. 113, 184 - 215 (2015)

DOI : 10.1080/00268976.2014.952696

Parts of Q-Chem use Armadillo 8.300.2 (tropical Shenanigans). http://arma.sourceforge.net/

Wavefunction Inc. Sales: sales@wavefun.com Irvine CA Support: support@wavefun.com Web: www.wavefun.com 


\section{References:}

1. Brandt, D.; Bellosta, V.; Cossy, J. Org. Lett. 2012, 14, 5594.

2. Satyanarayana, S.; Reddy, B. V. S.; Narender, R. Tetrahedron Lett. 2014, 55, 6027.

3. $\quad$ Singh, O. V.; Han, H. Org. Lett. 2007, 9, 4801.

4. $\quad$ Lin, H.; Liu, Y.; Wu, Z. L. Chem Commun (Camb) 2011, 47, 2610.

5. Ickes, A. R.; Ensign, S. C.; Gupta, A. K.; Hull, K. L. J. Am. Chem. Soc. 2014, 136, 11256.

6. Burton, H.; Ingold, C. K. J. Chem. Soc. 1928, 904.

7. Smith, A. B.; Condon, S. M.; McCauley, J. A.; Leazer, J. L.; Leahy, J. W.; Maleczka, R. E. J. Am. Chem. Soc. 1997, 119, 962.

8. Hart, H.; Brewbaker, J. L. J. Am. Chem. Soc. 1969, 91, 716.

9. Ao, Y.-F.; Leng, D.-H.; Wang, D.-X.; Zhao, L.; Wang, M.-X. Tetrahedron 2014, 70, 4309.

10. Tony, K. A.; Li, X.; Dabideen, D.; Li, J.; Mootoo, D. R. Organic \& Biomolecular Chemistry 2008, 6, 1165. 


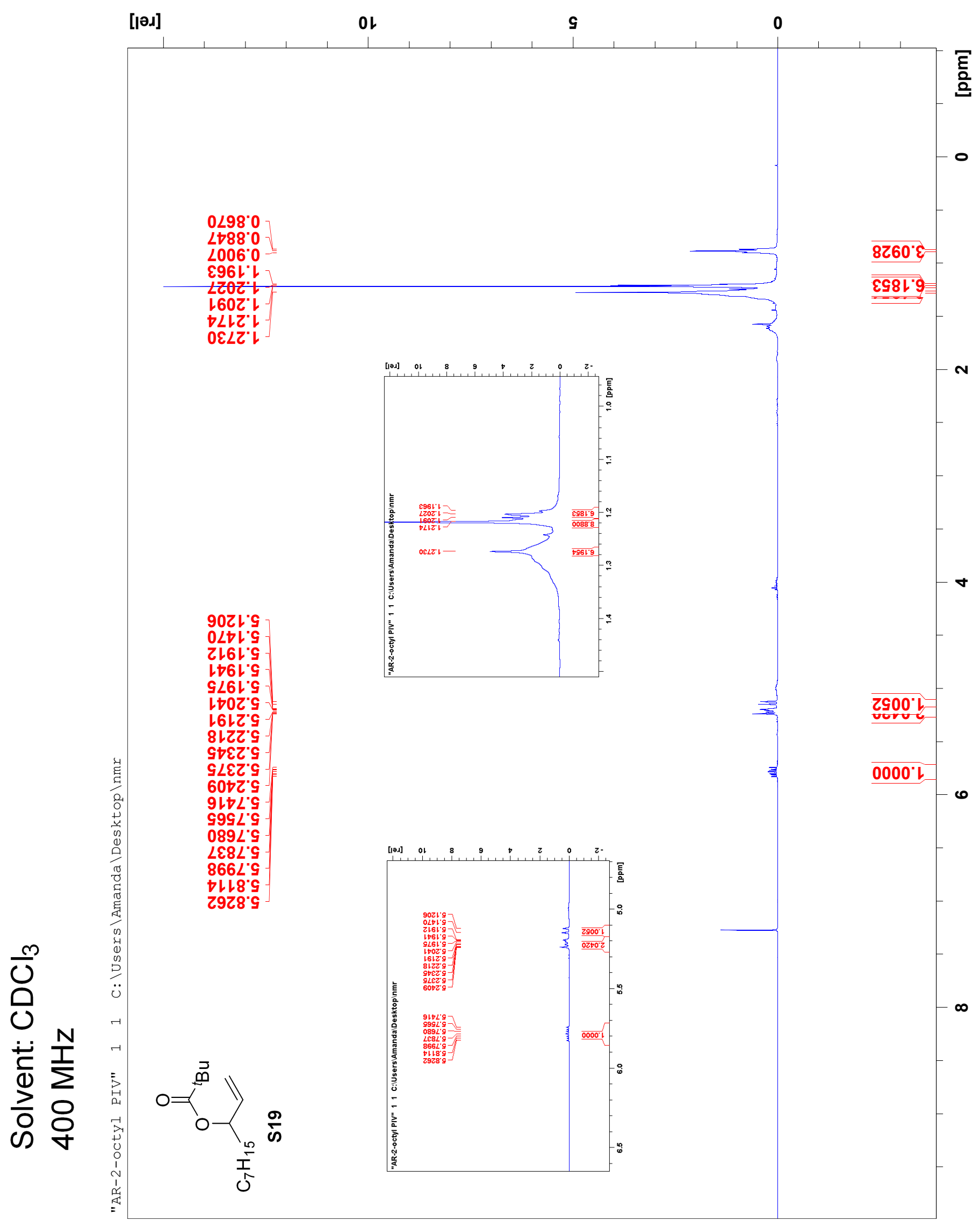




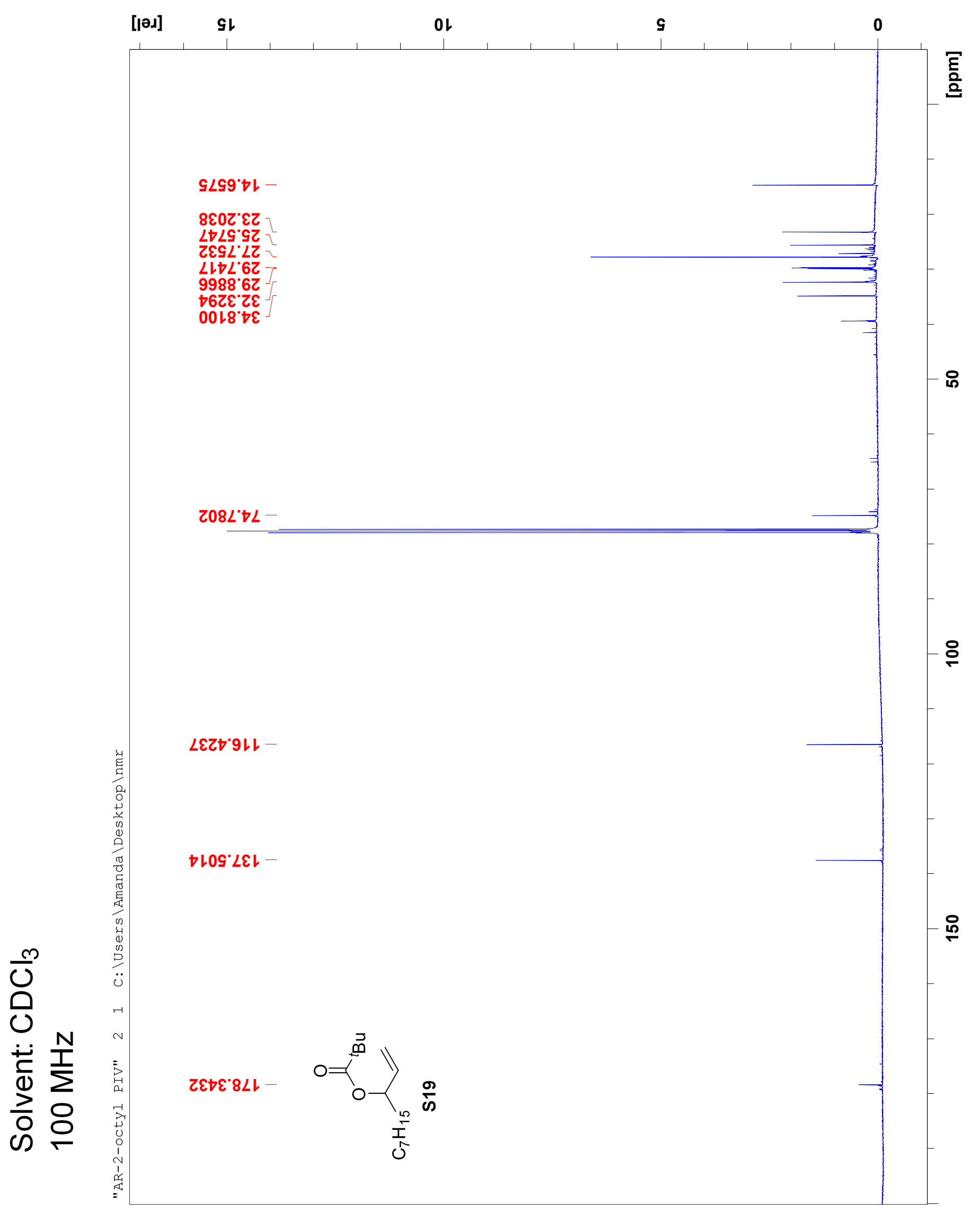














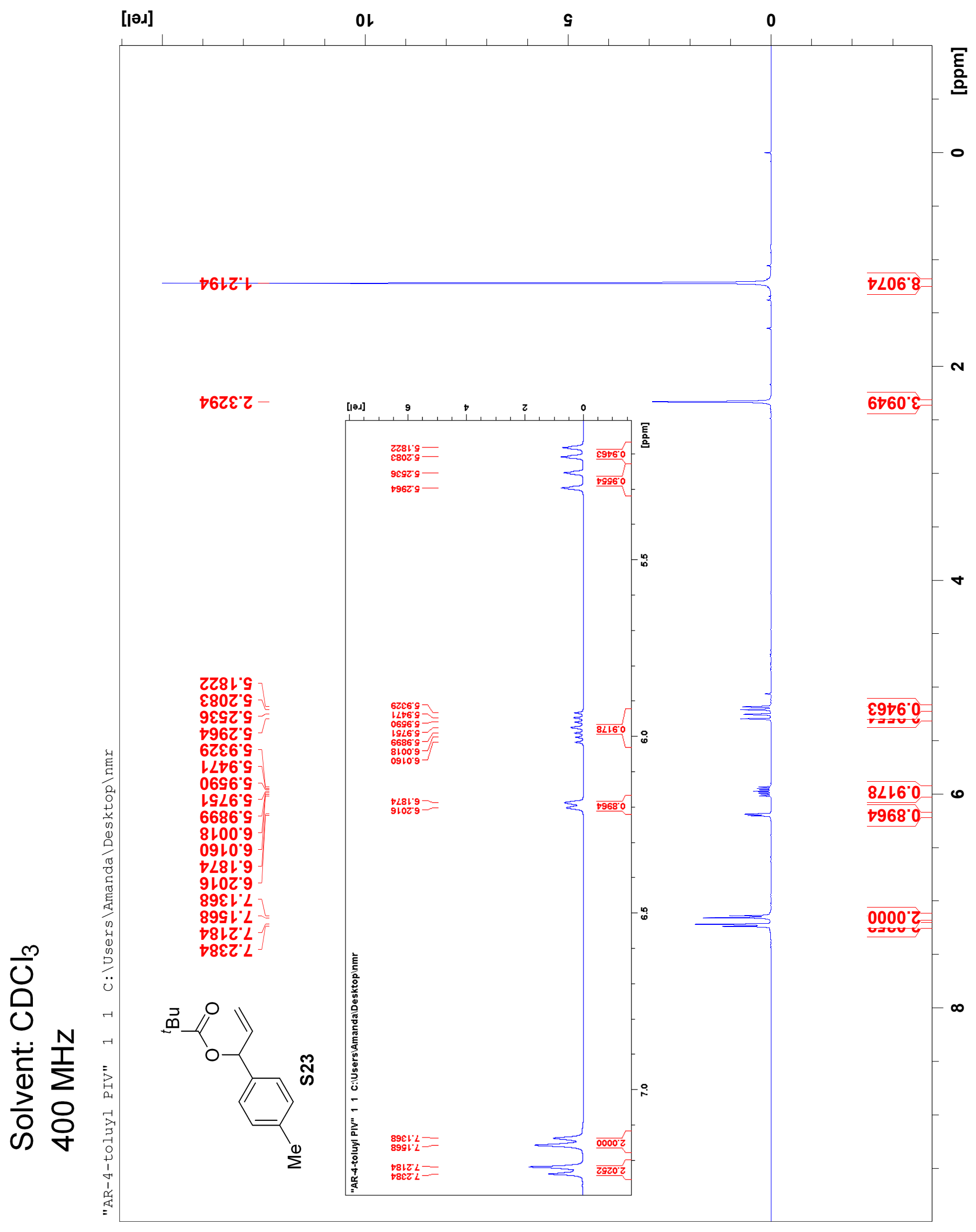




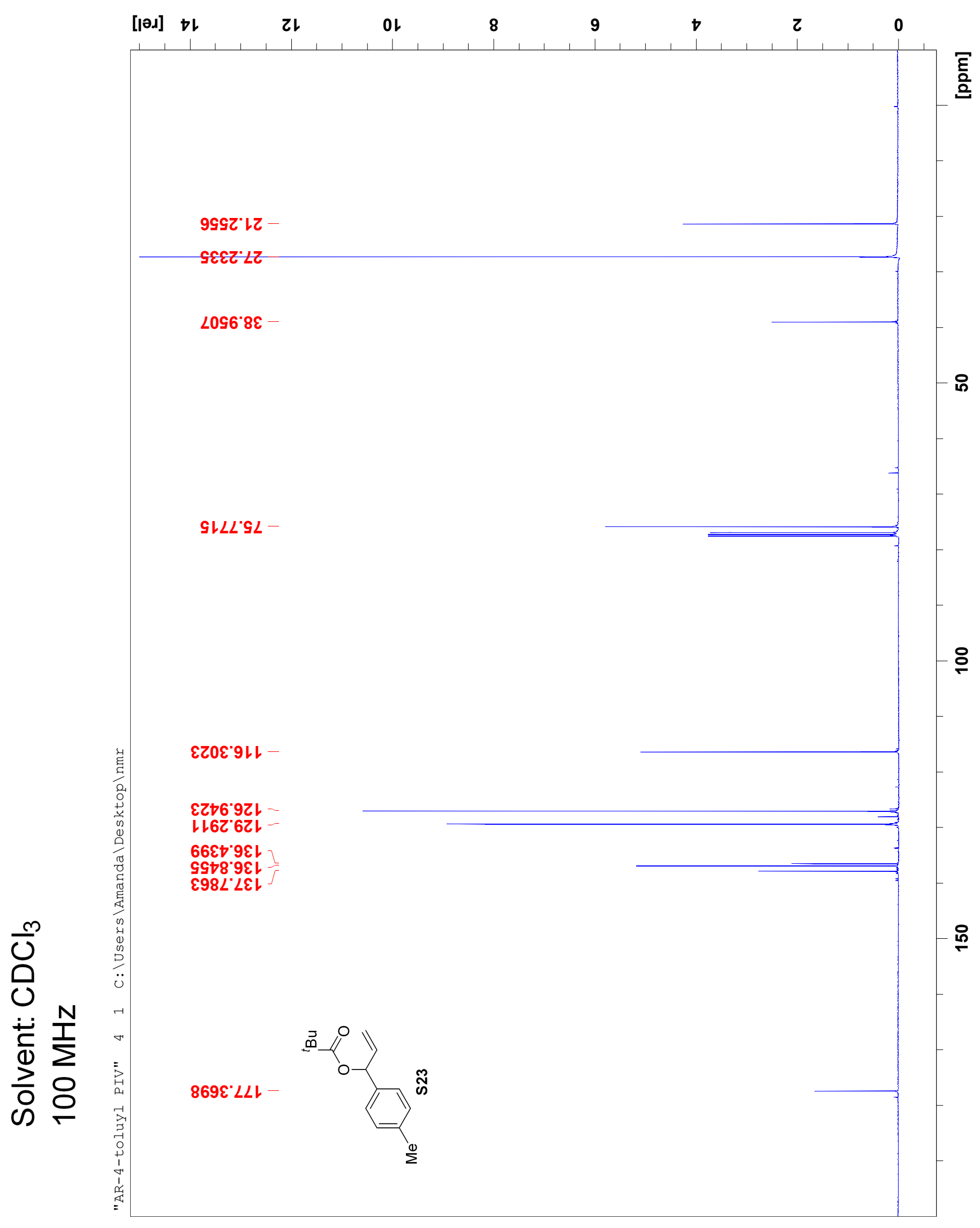




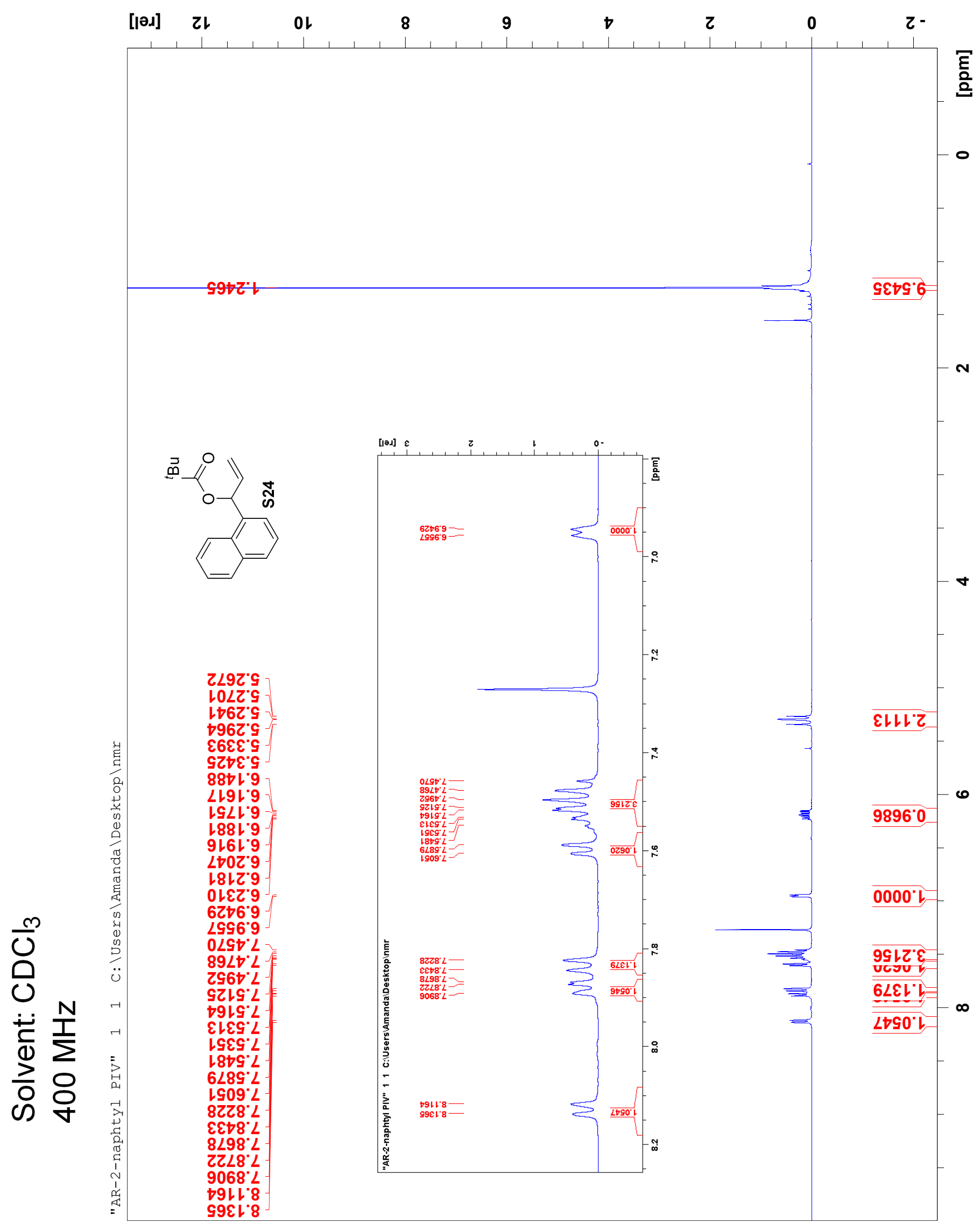




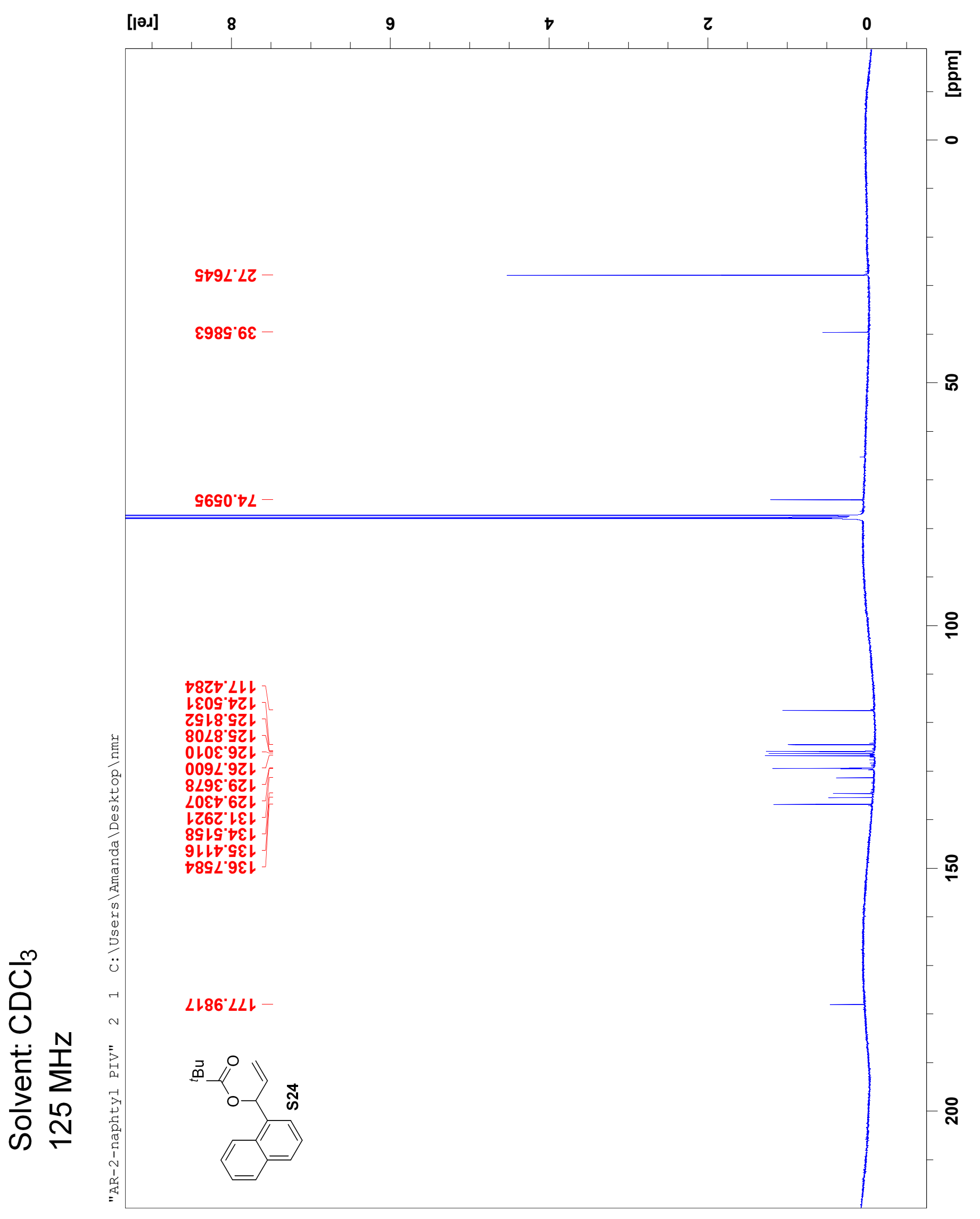




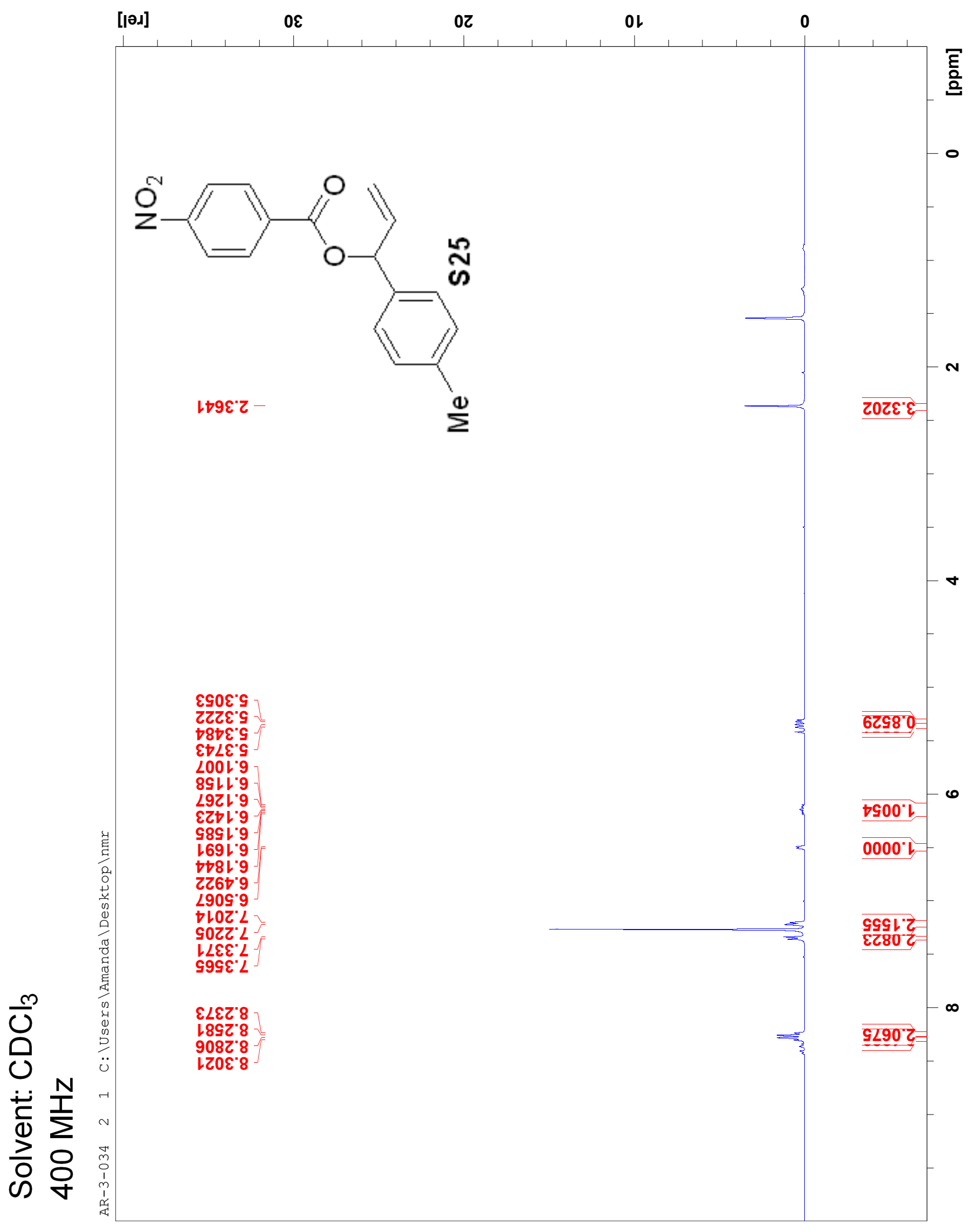




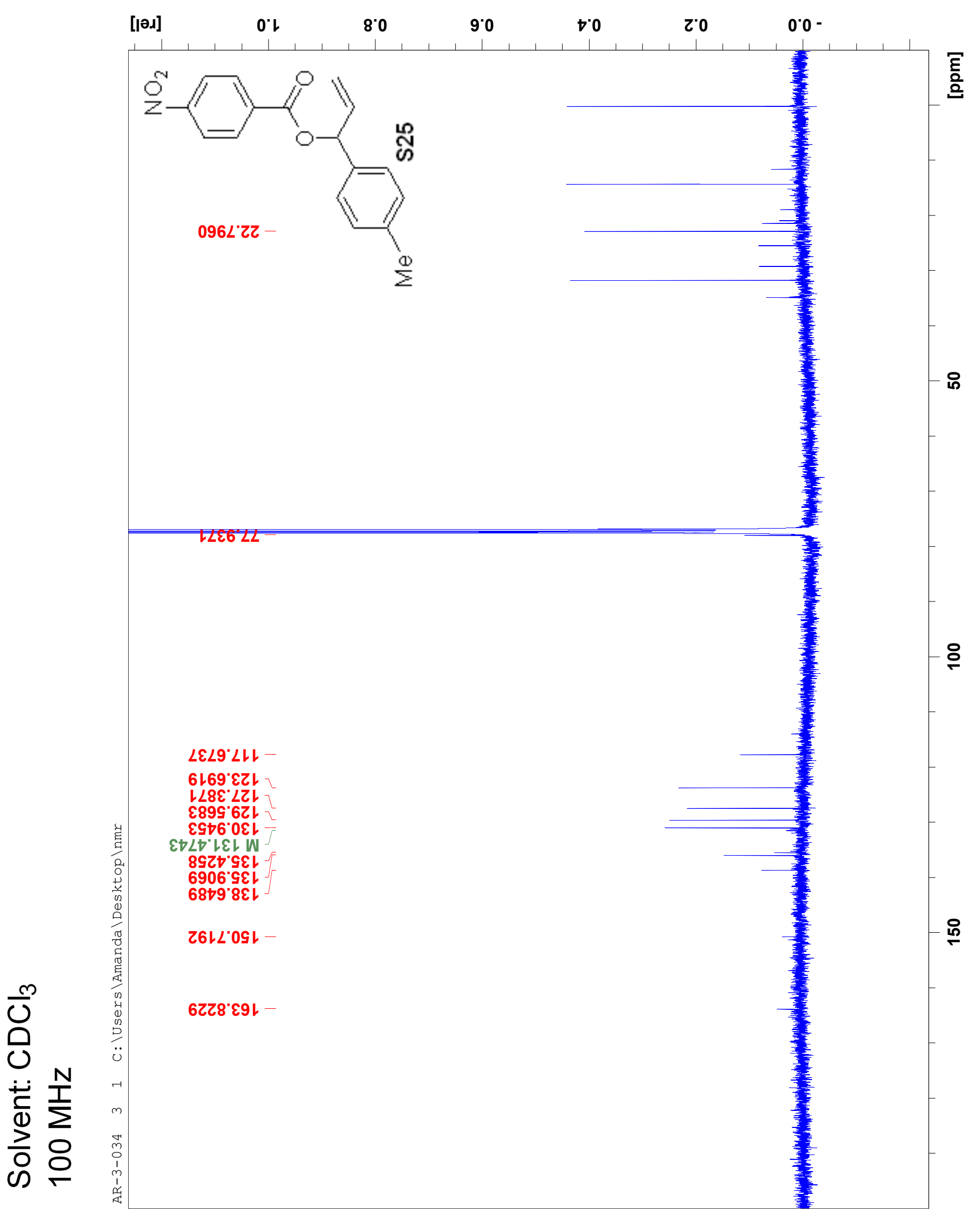




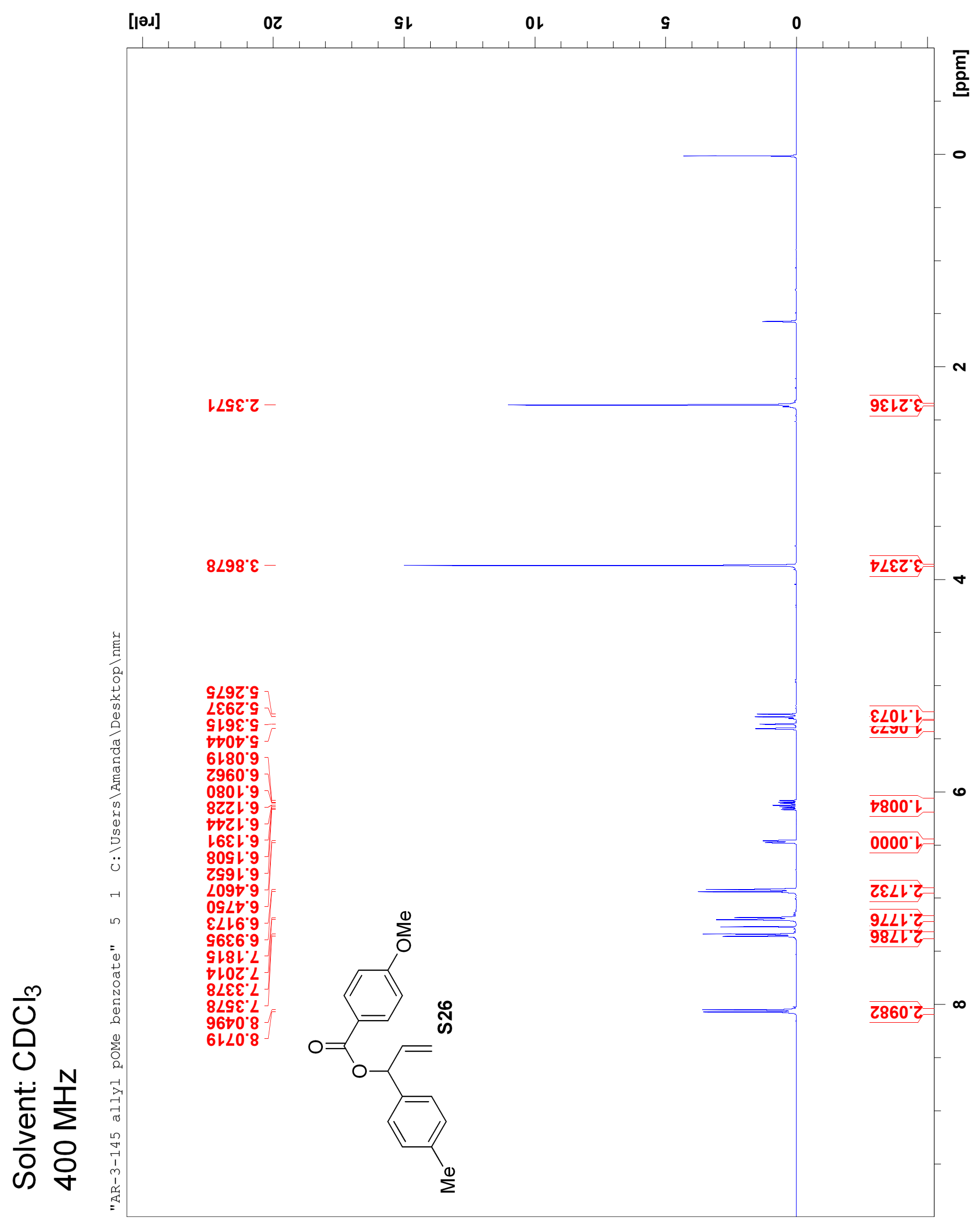




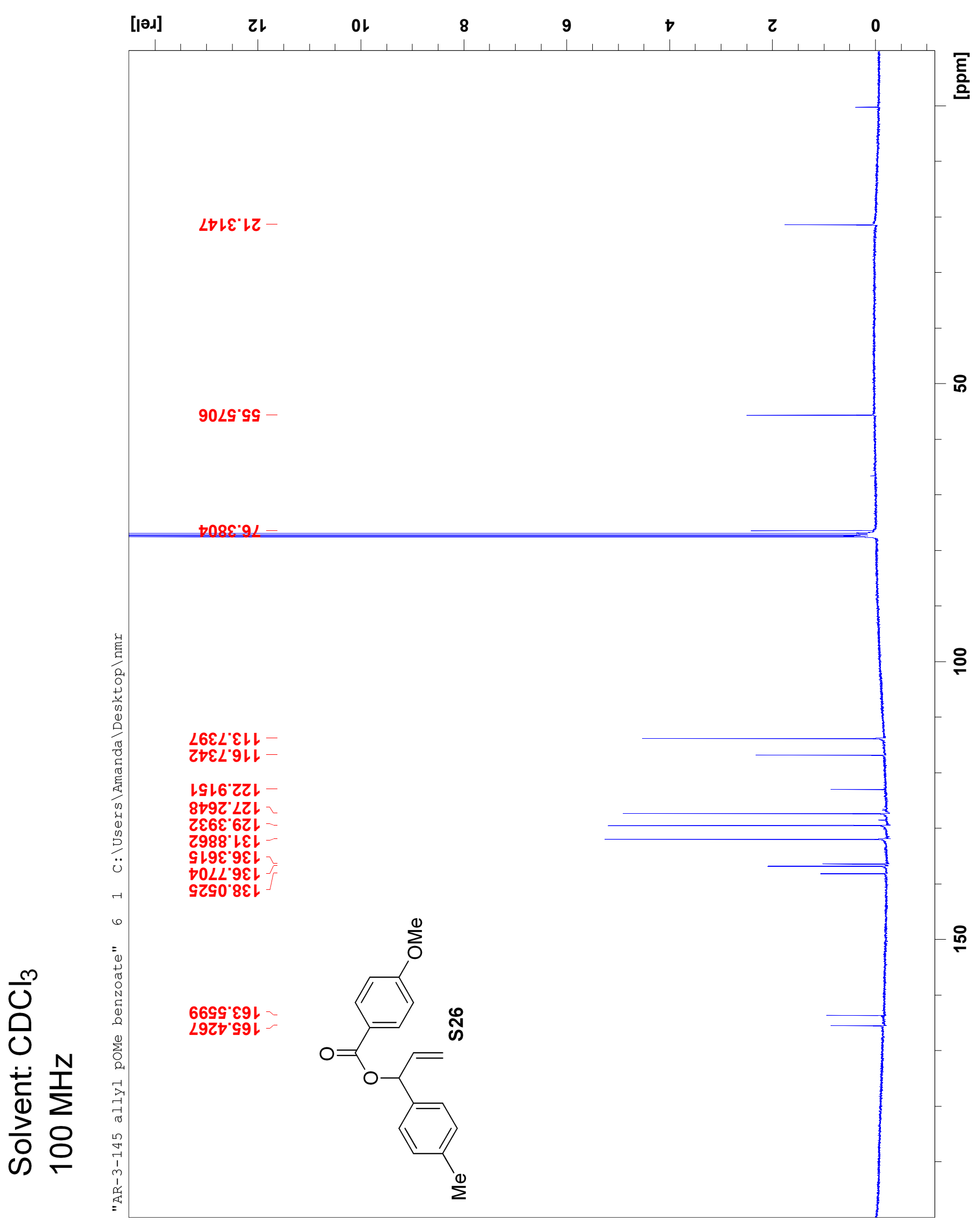




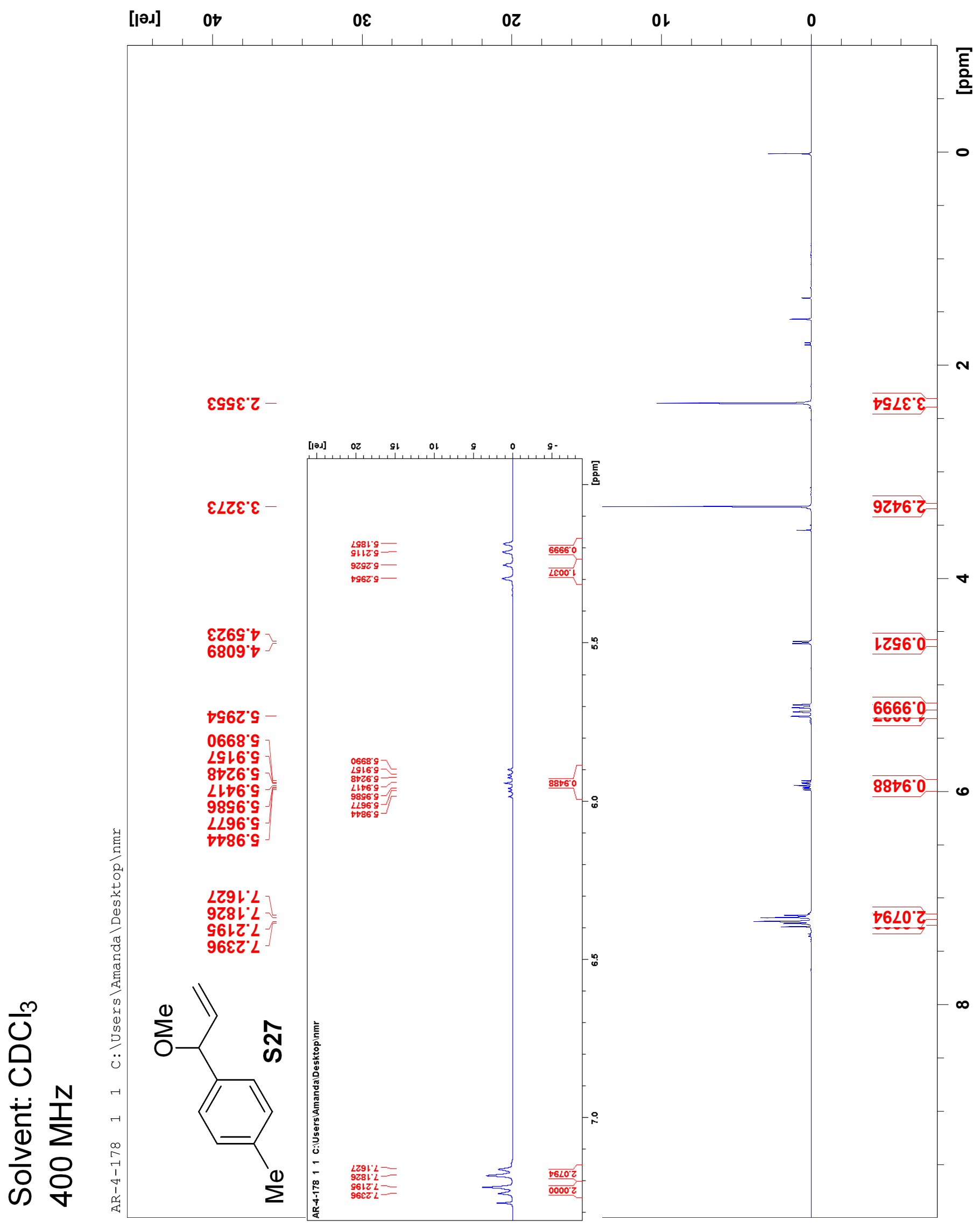




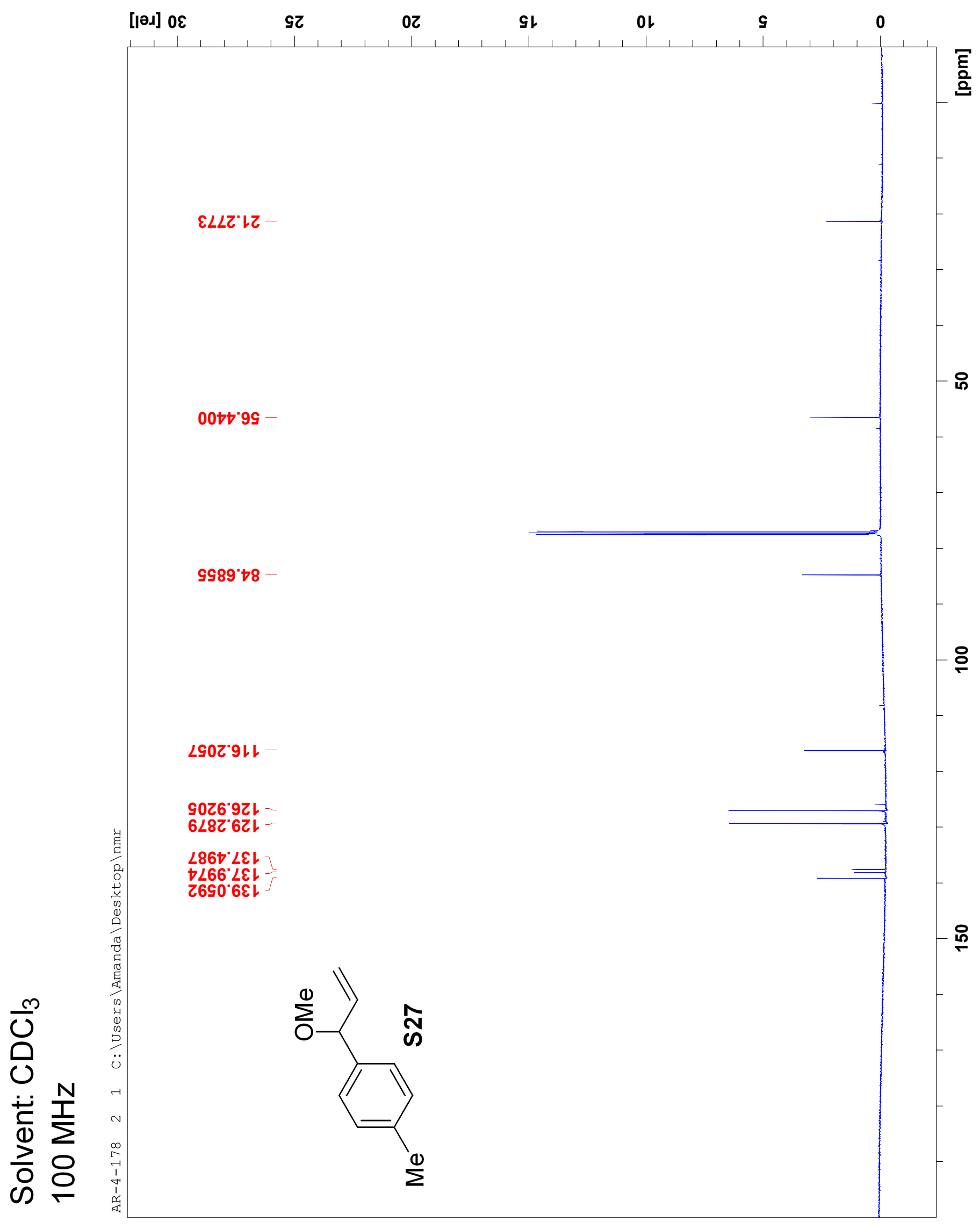




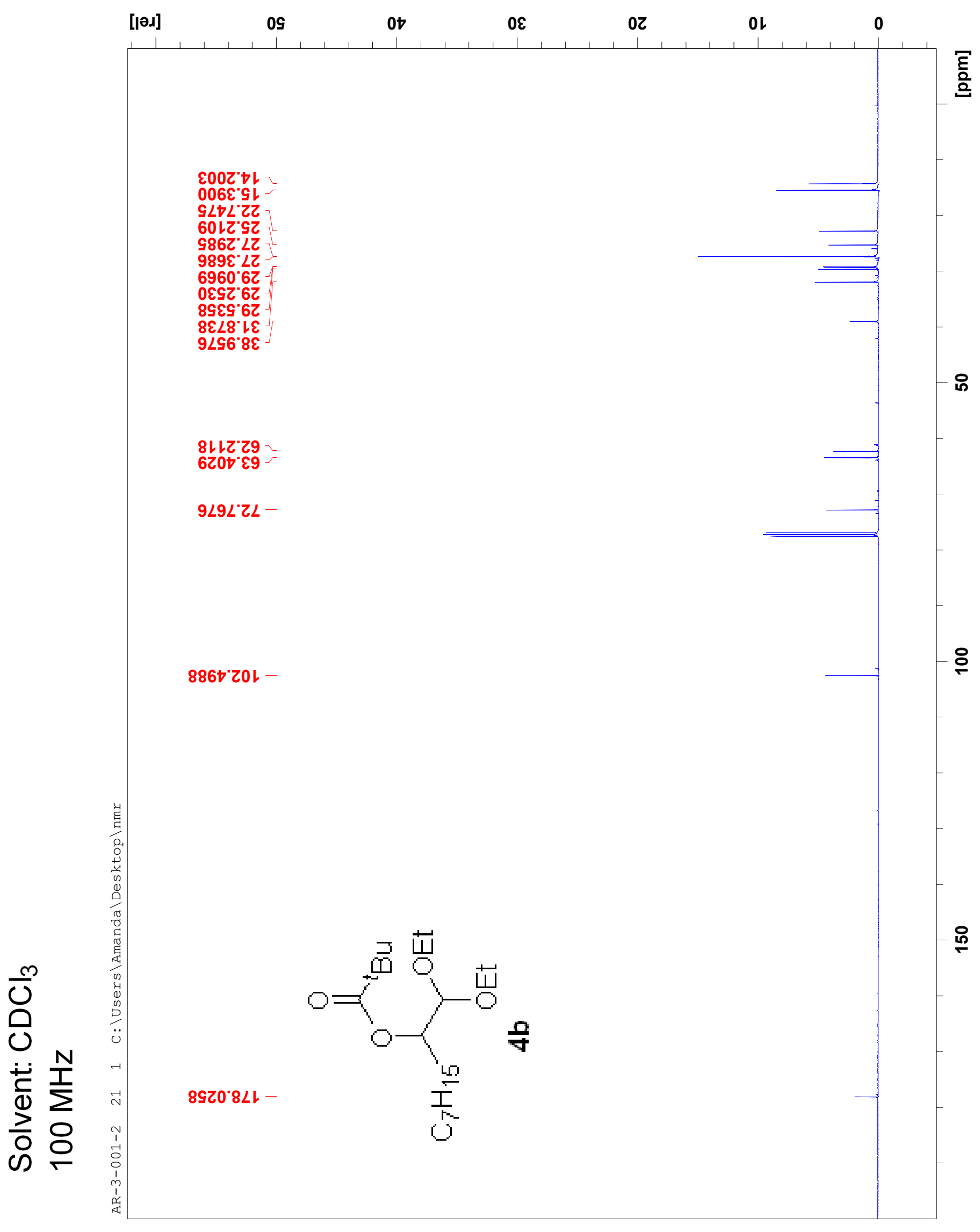




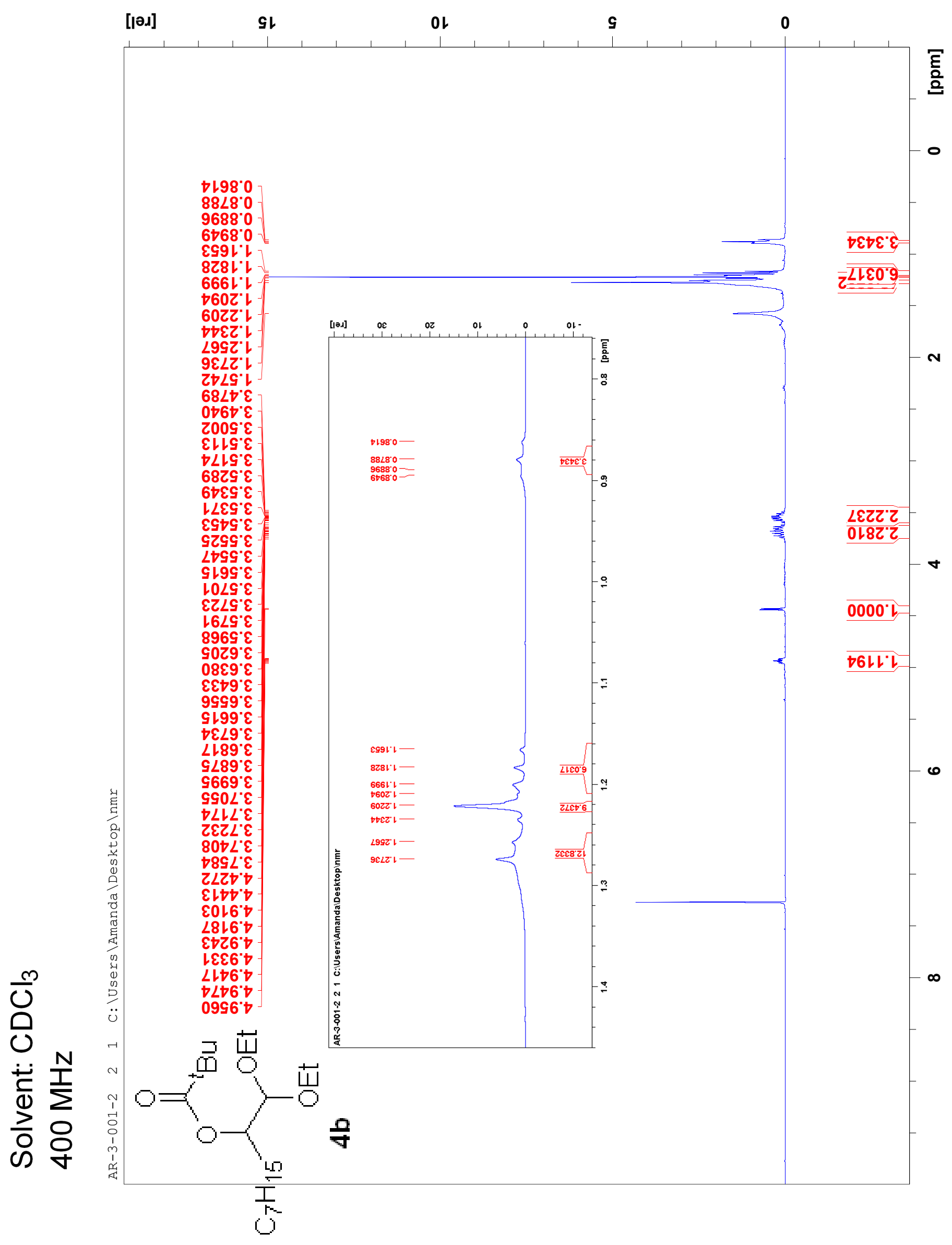




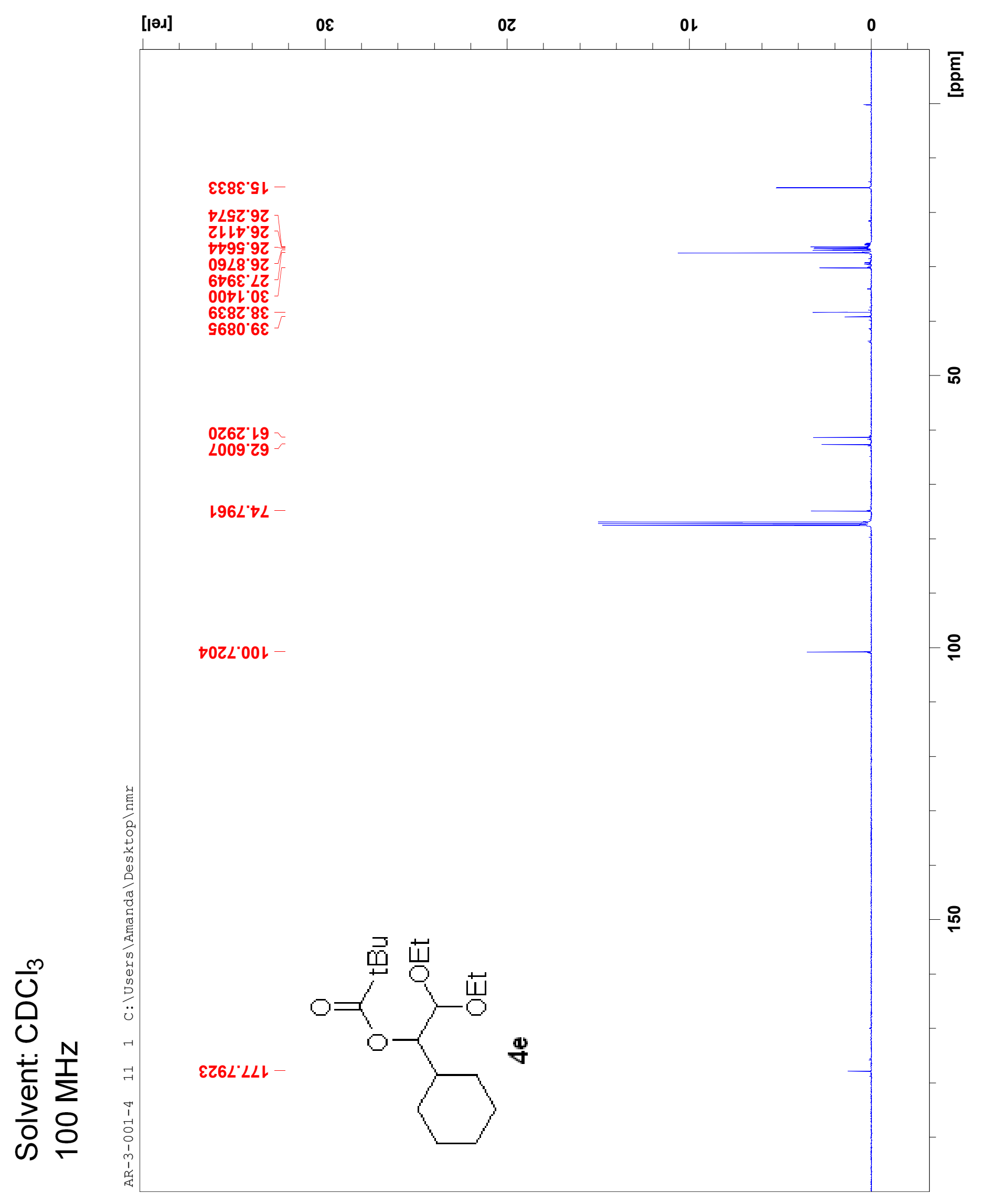




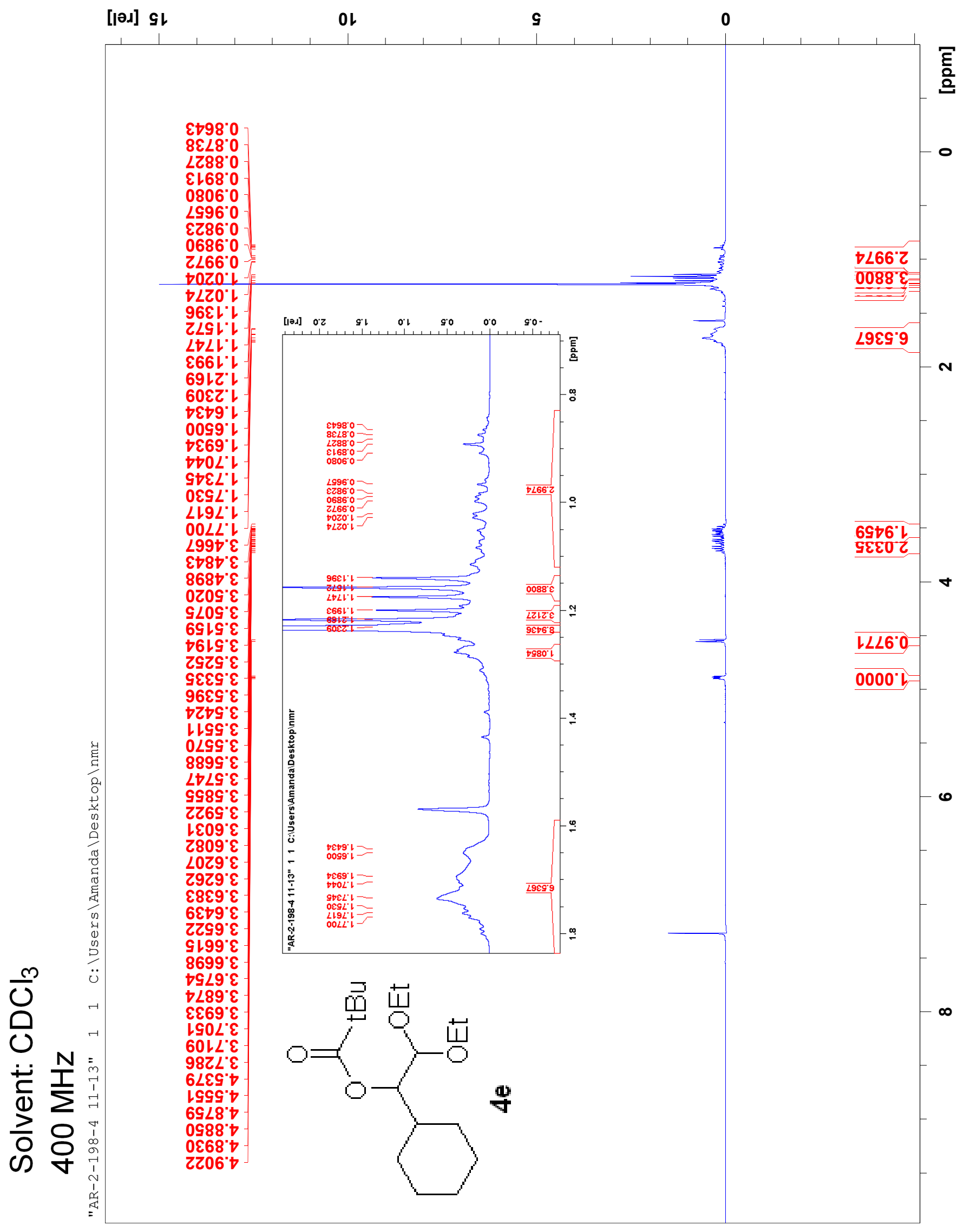




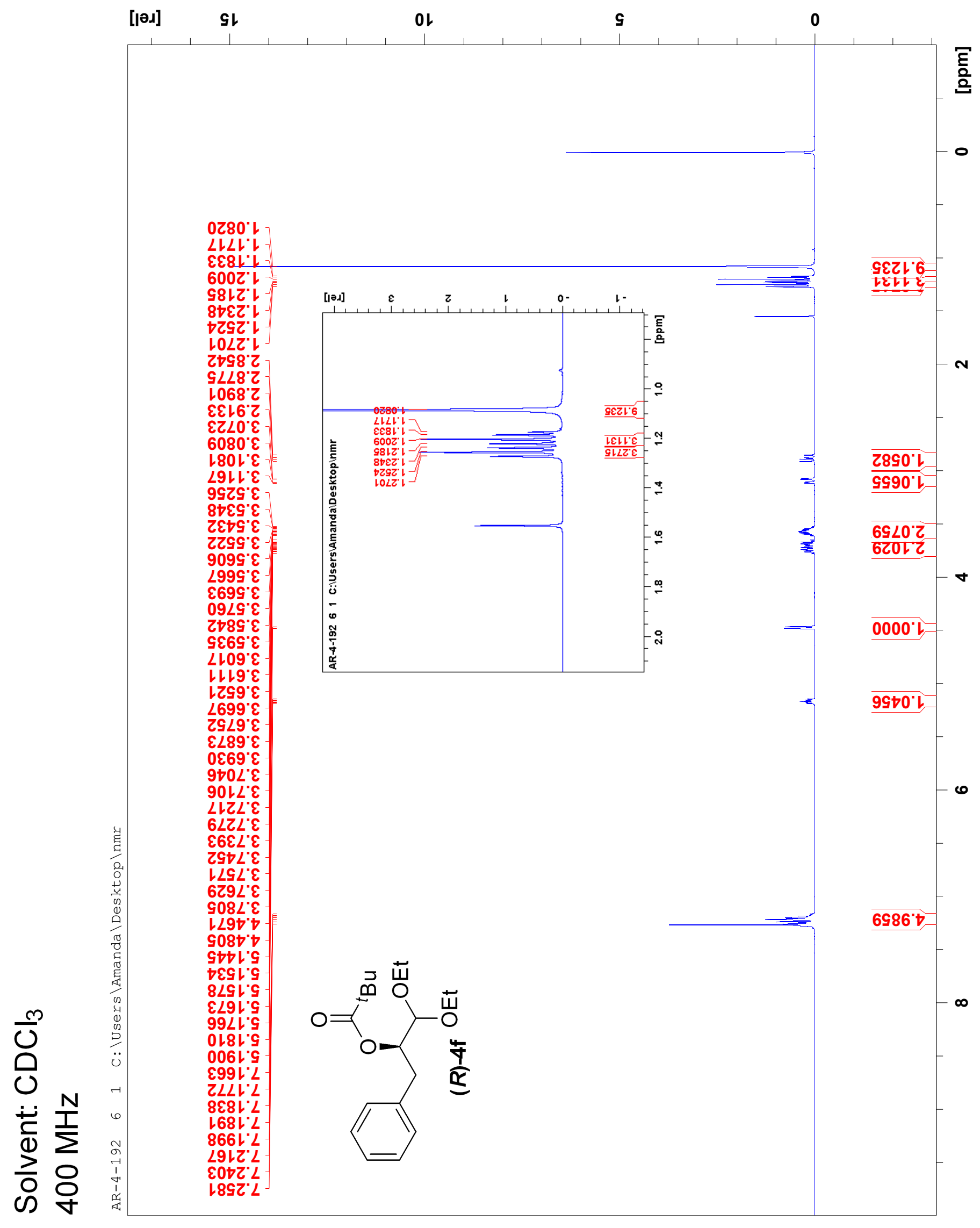




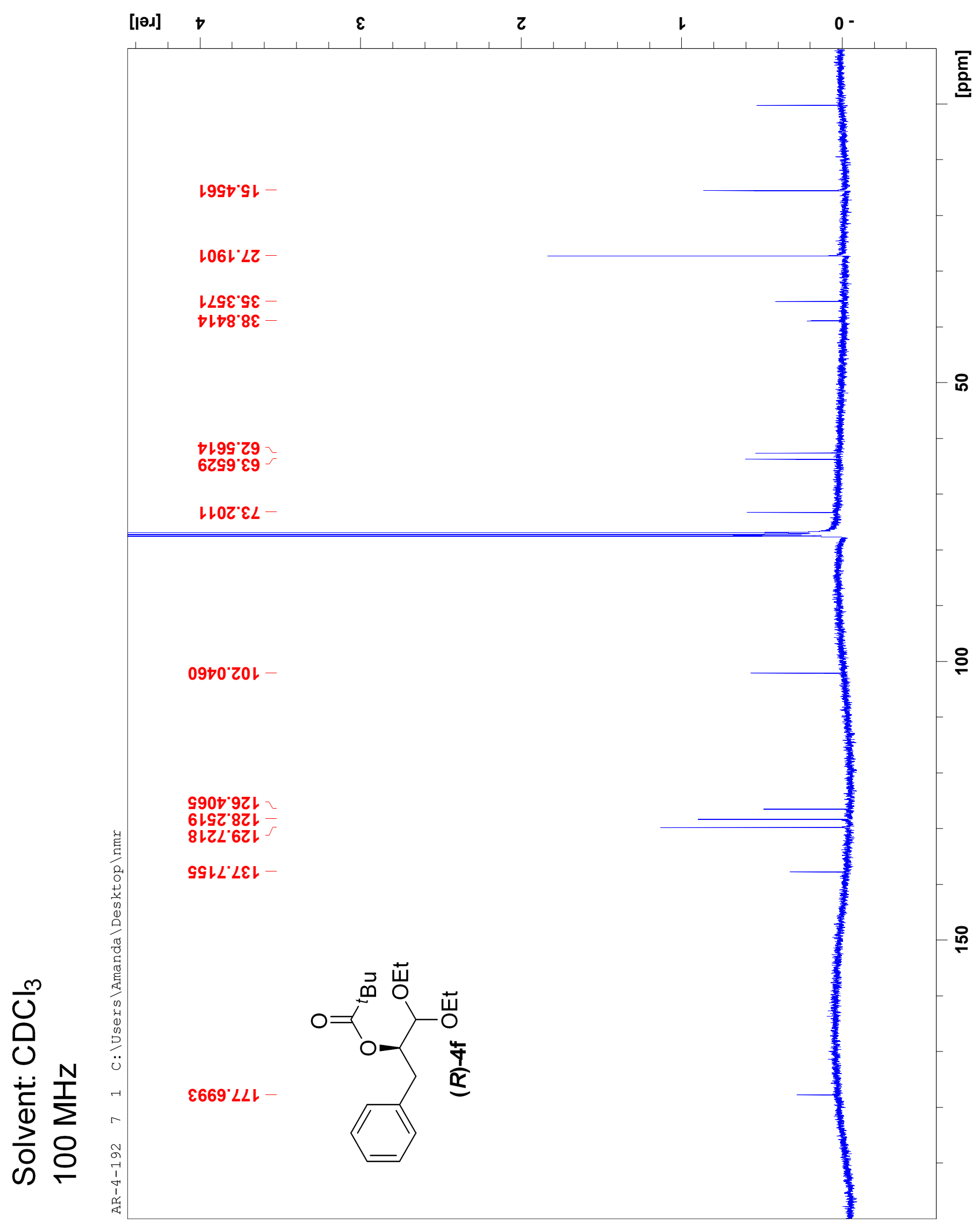




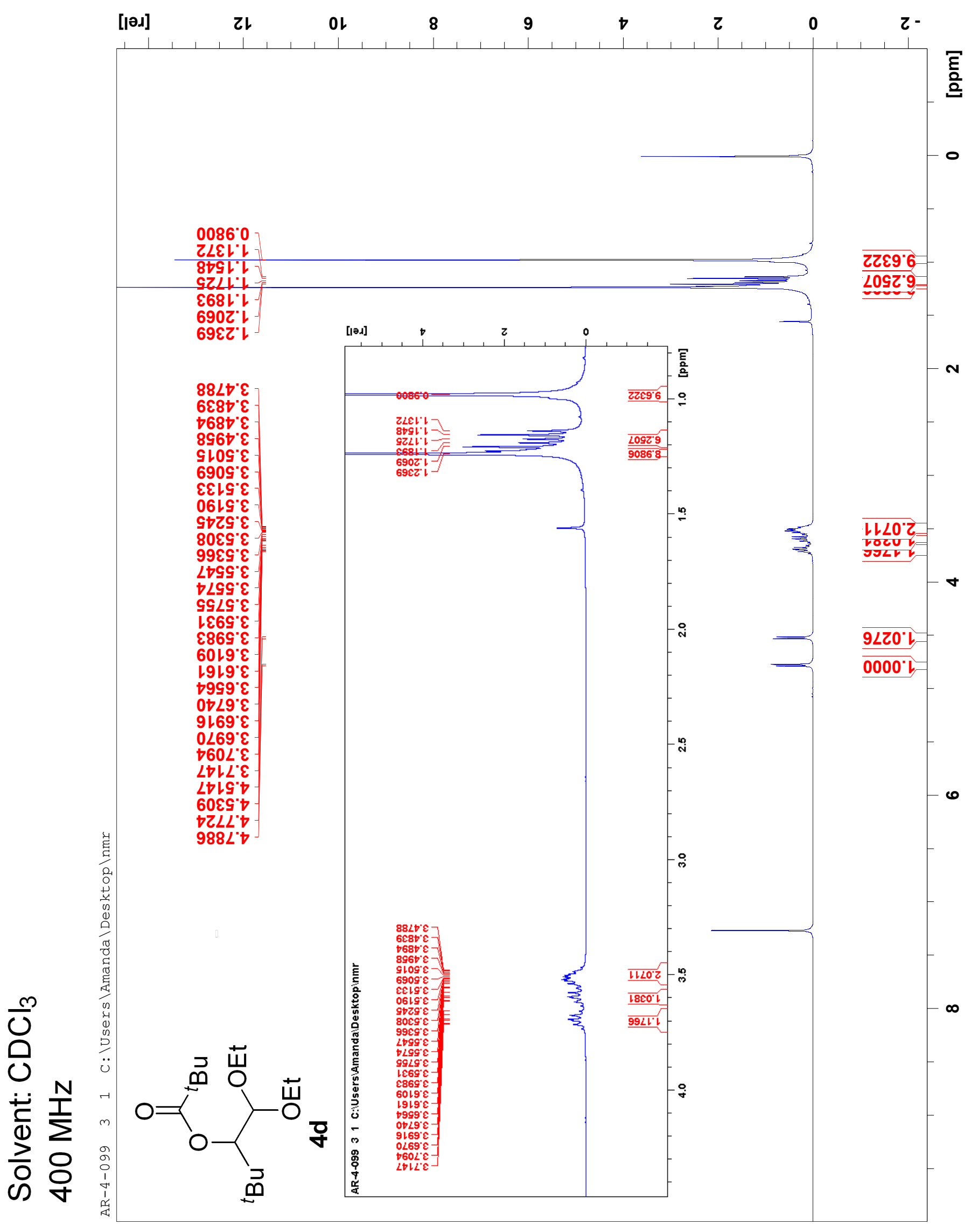




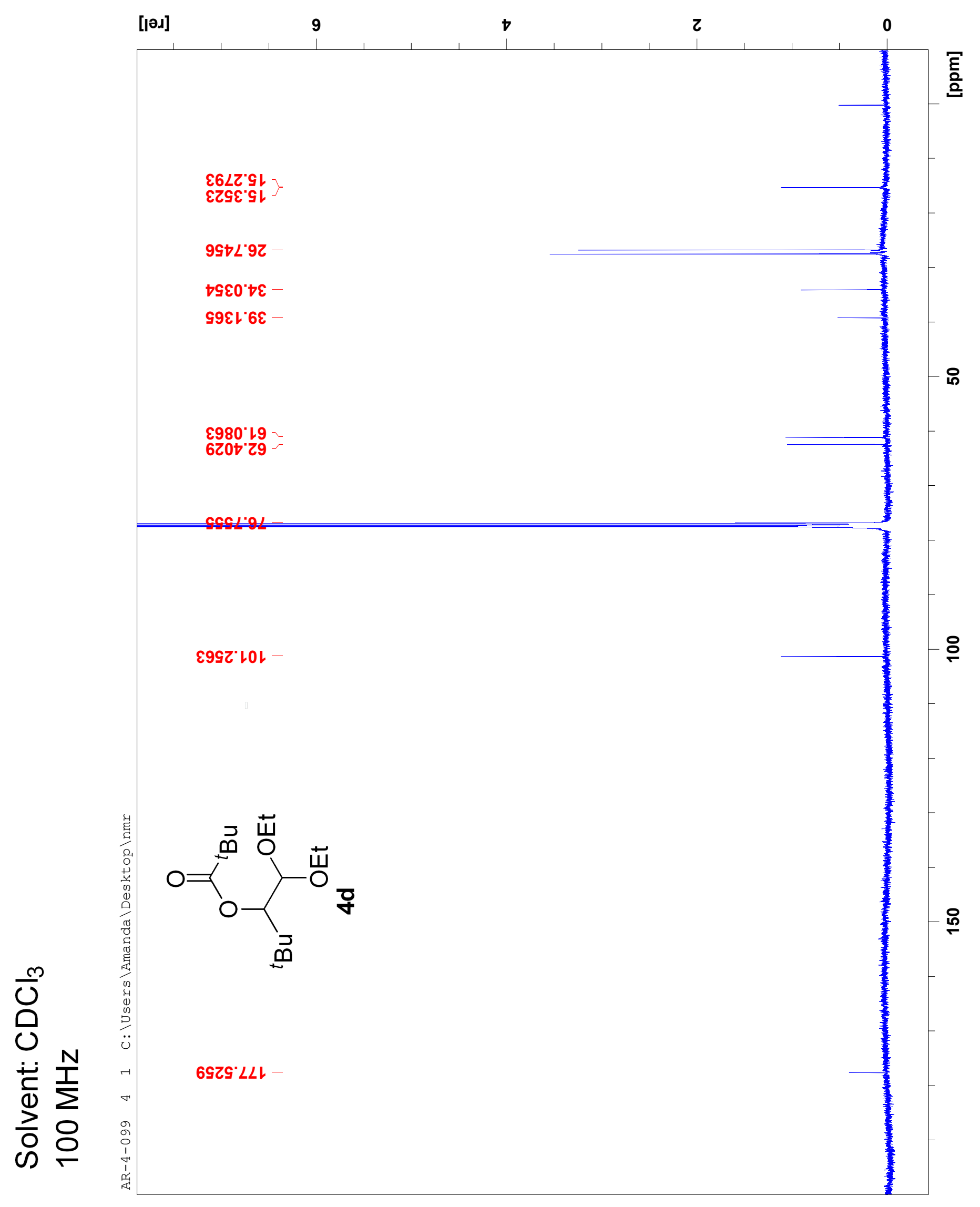




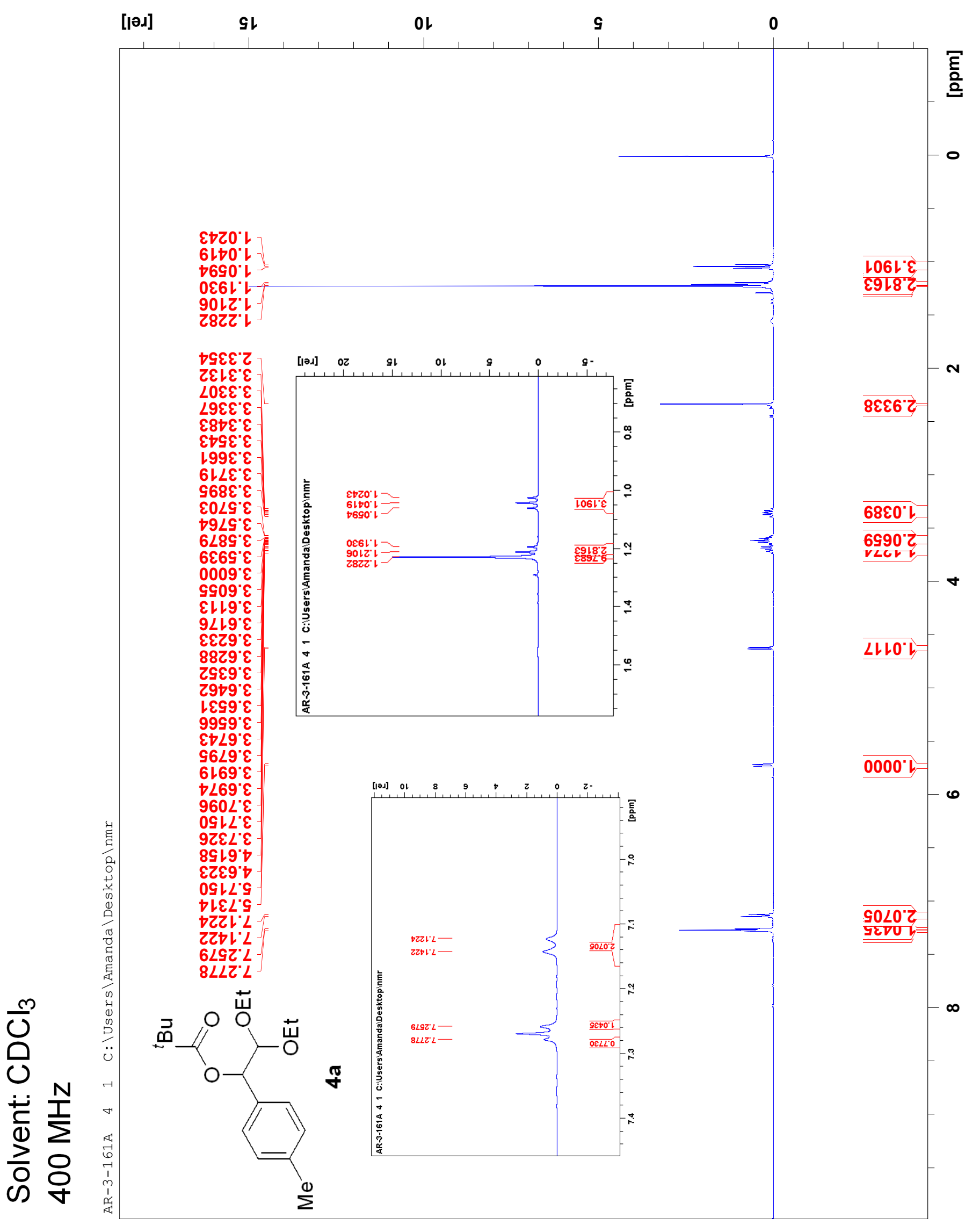




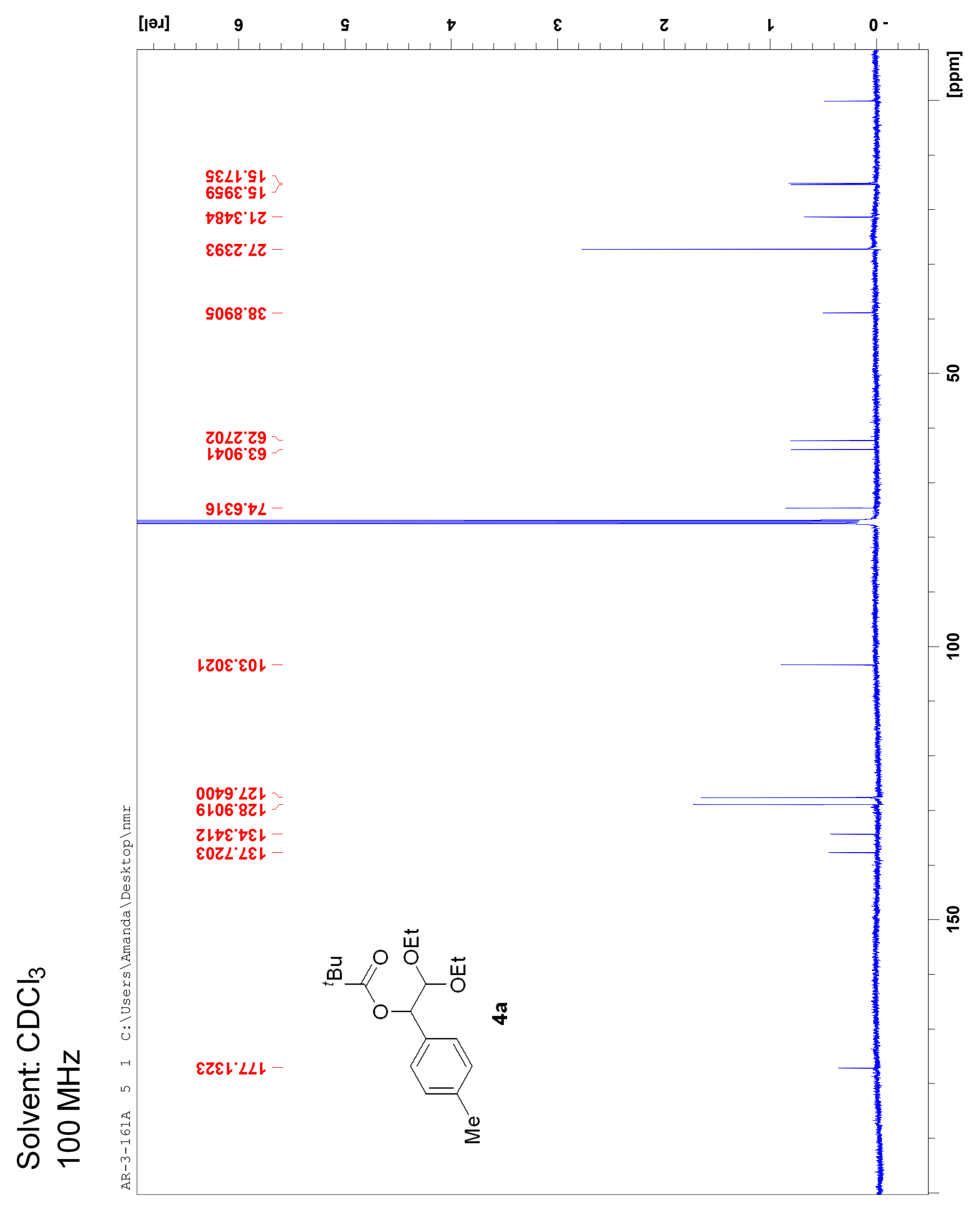














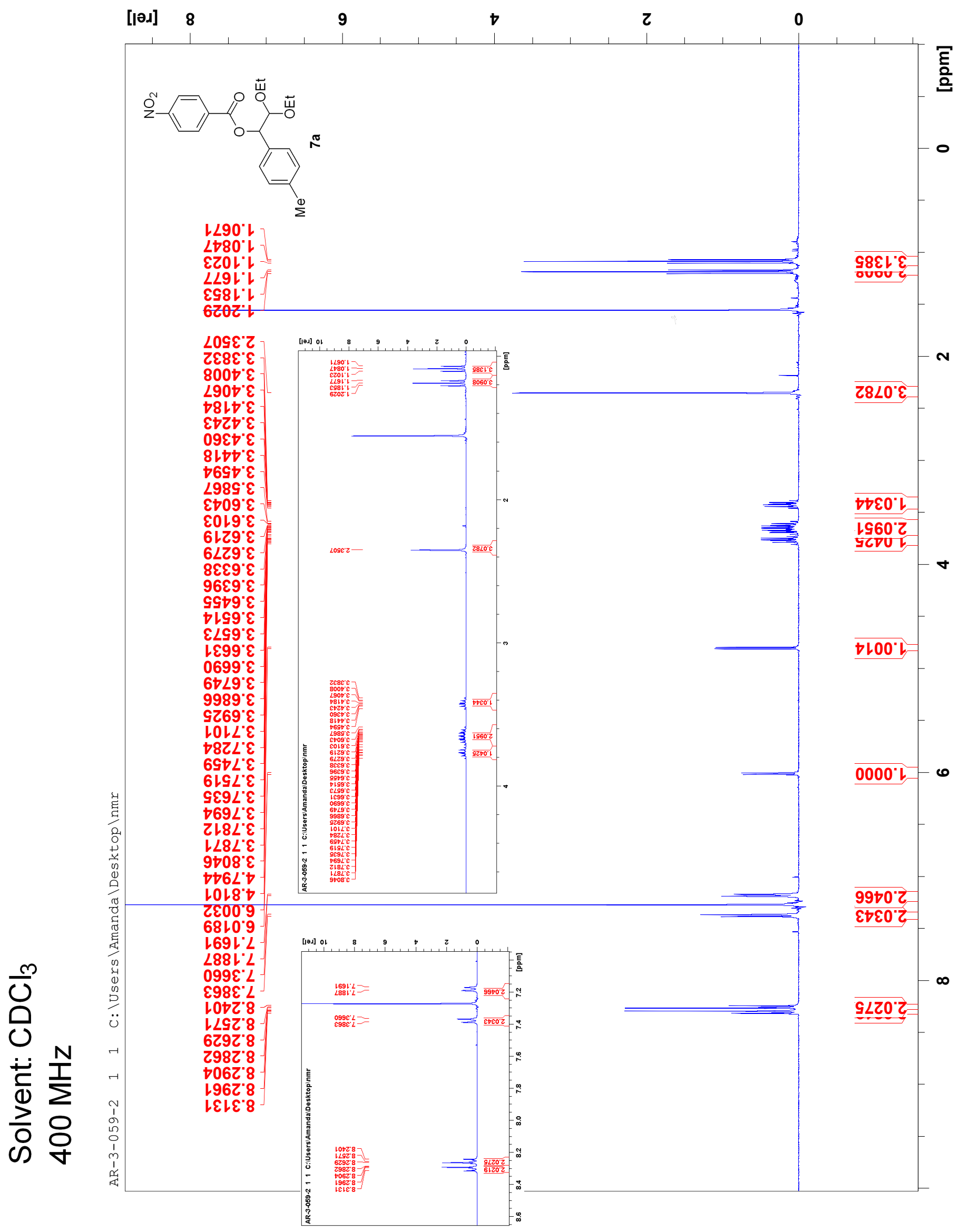




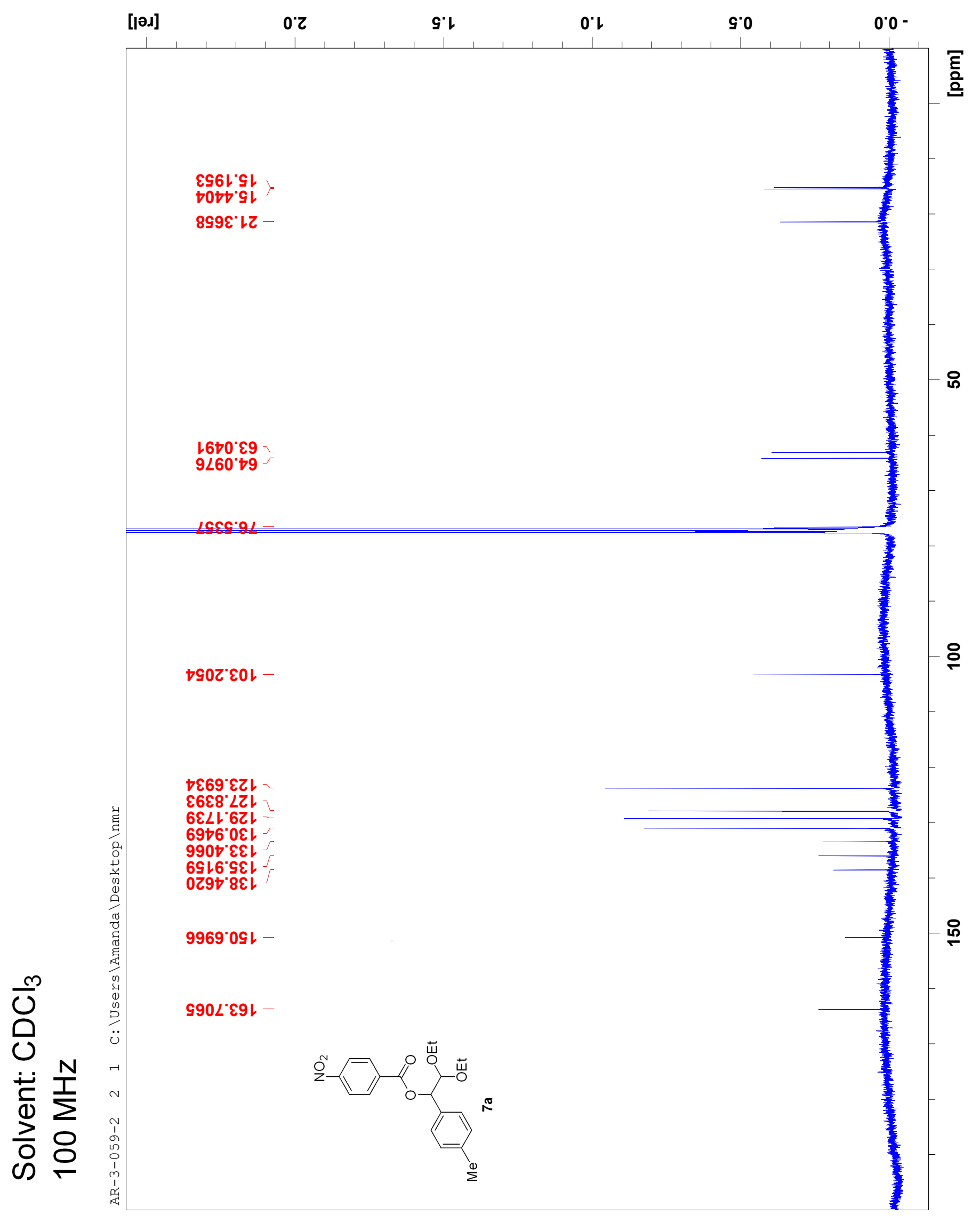




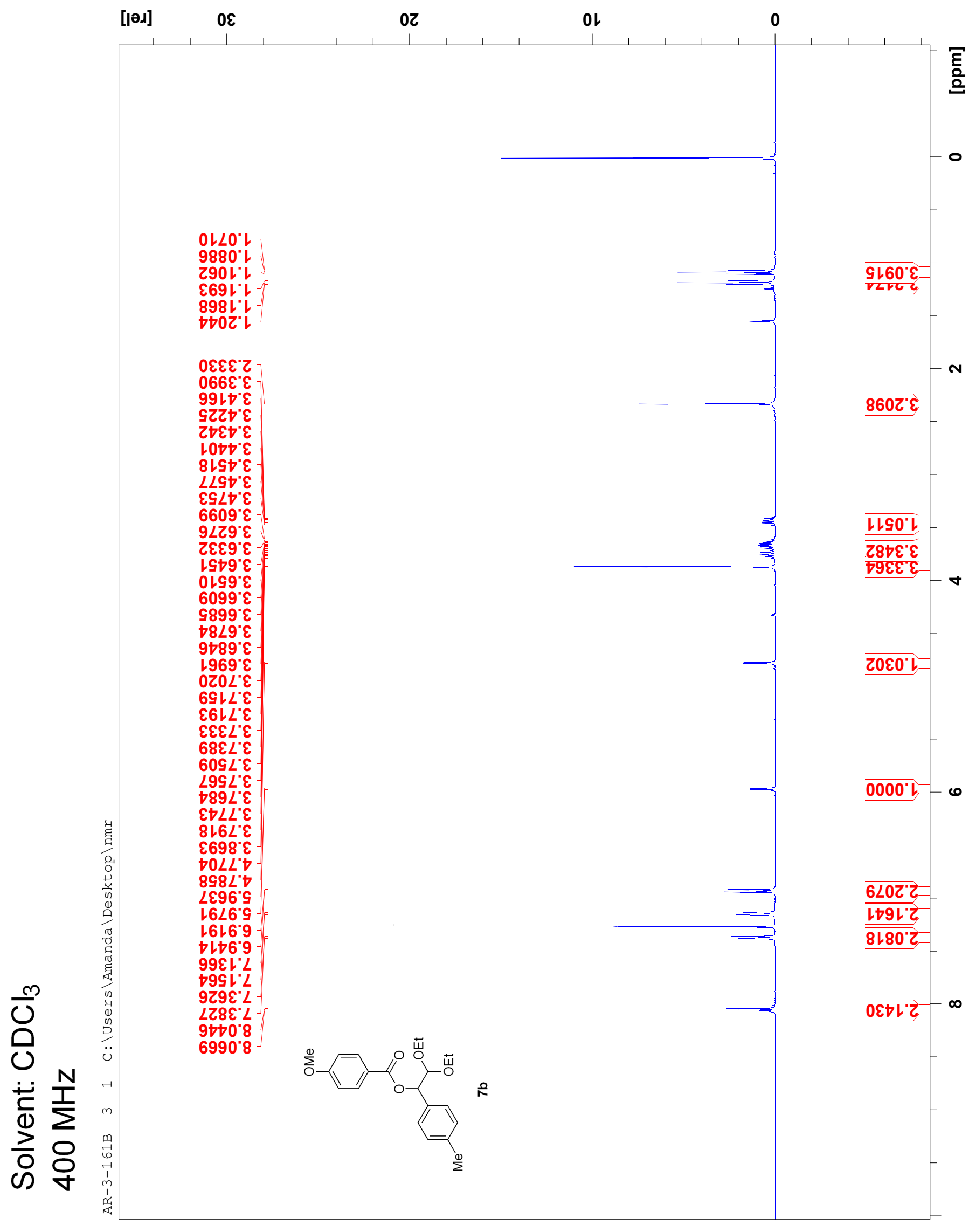




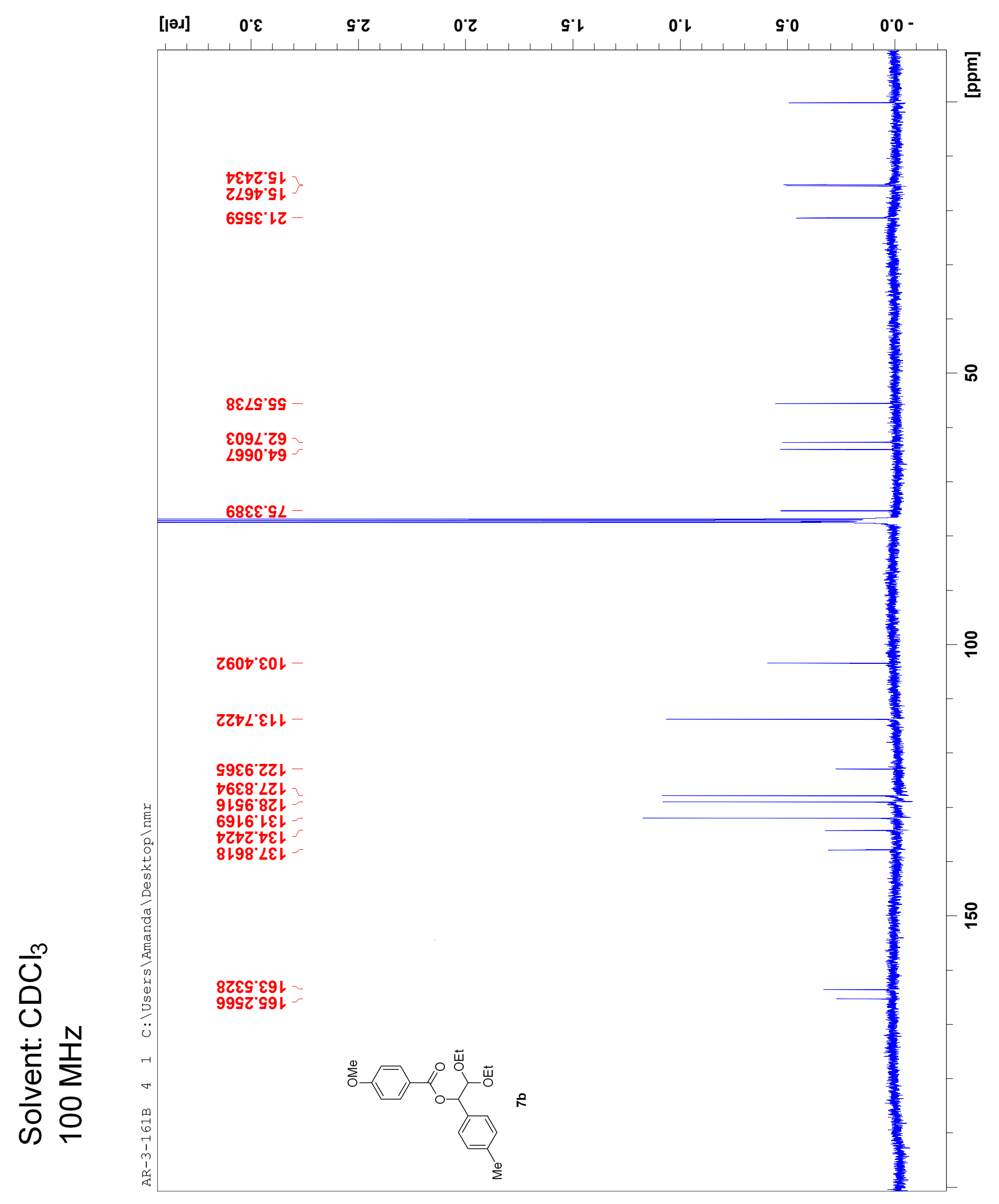




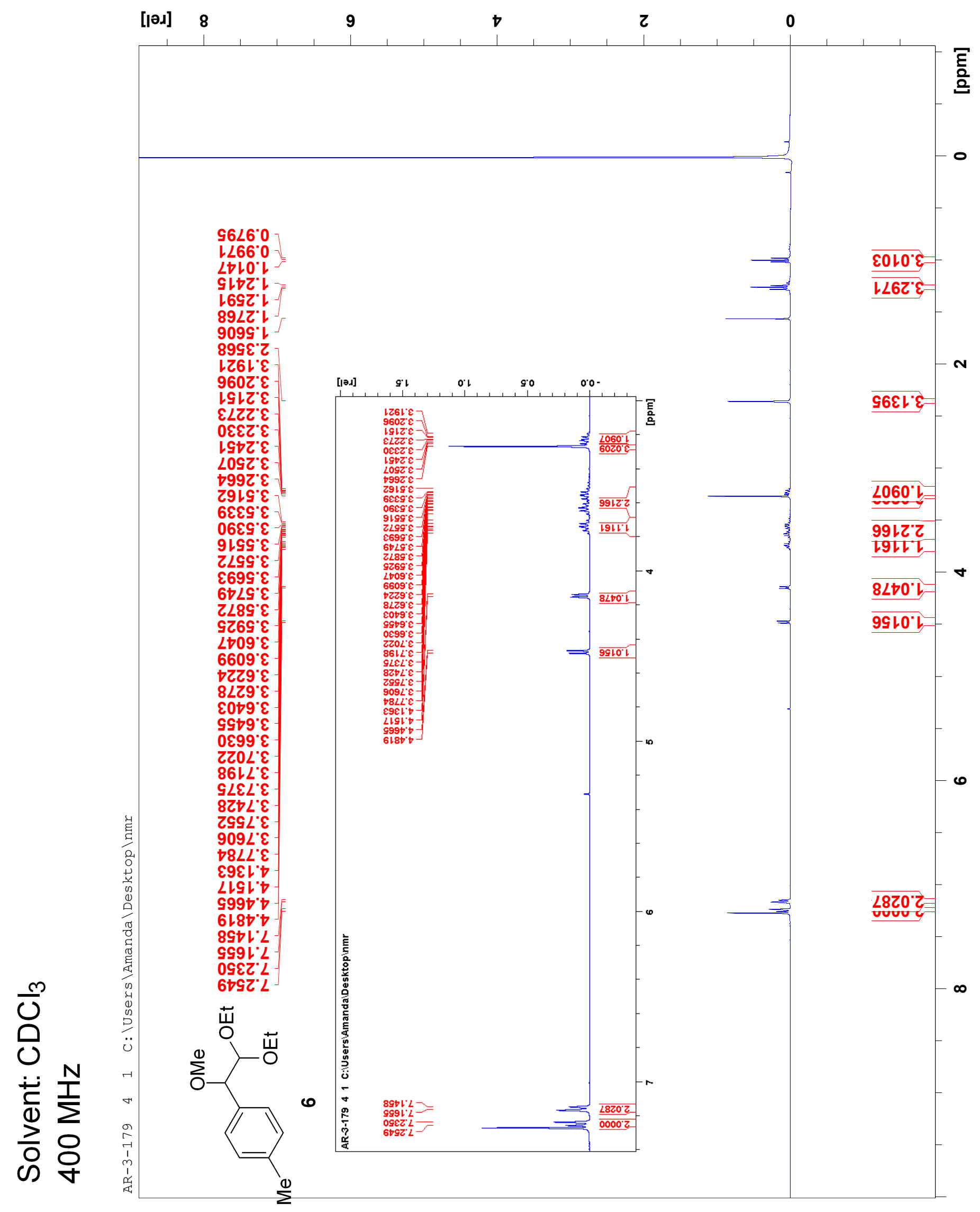









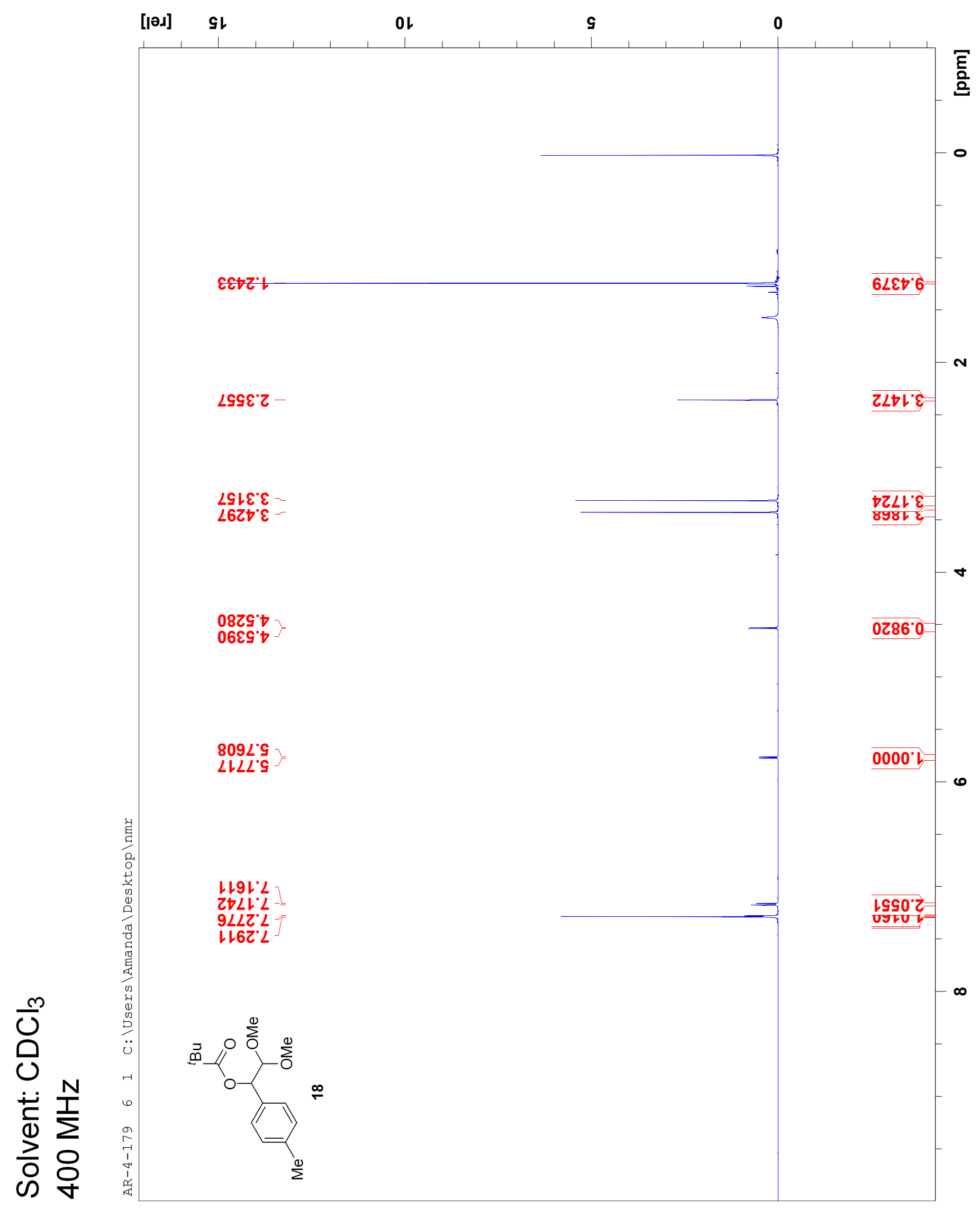




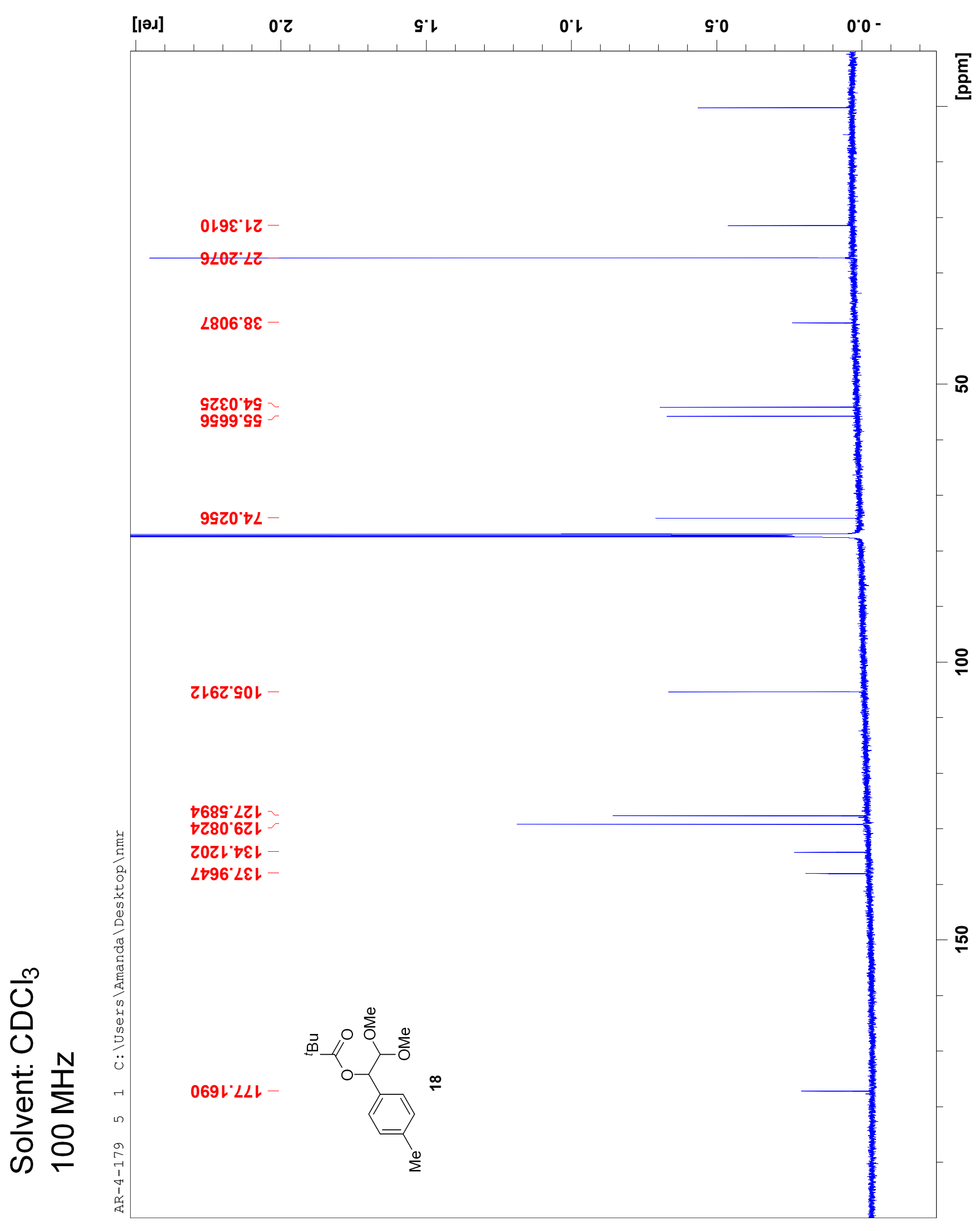




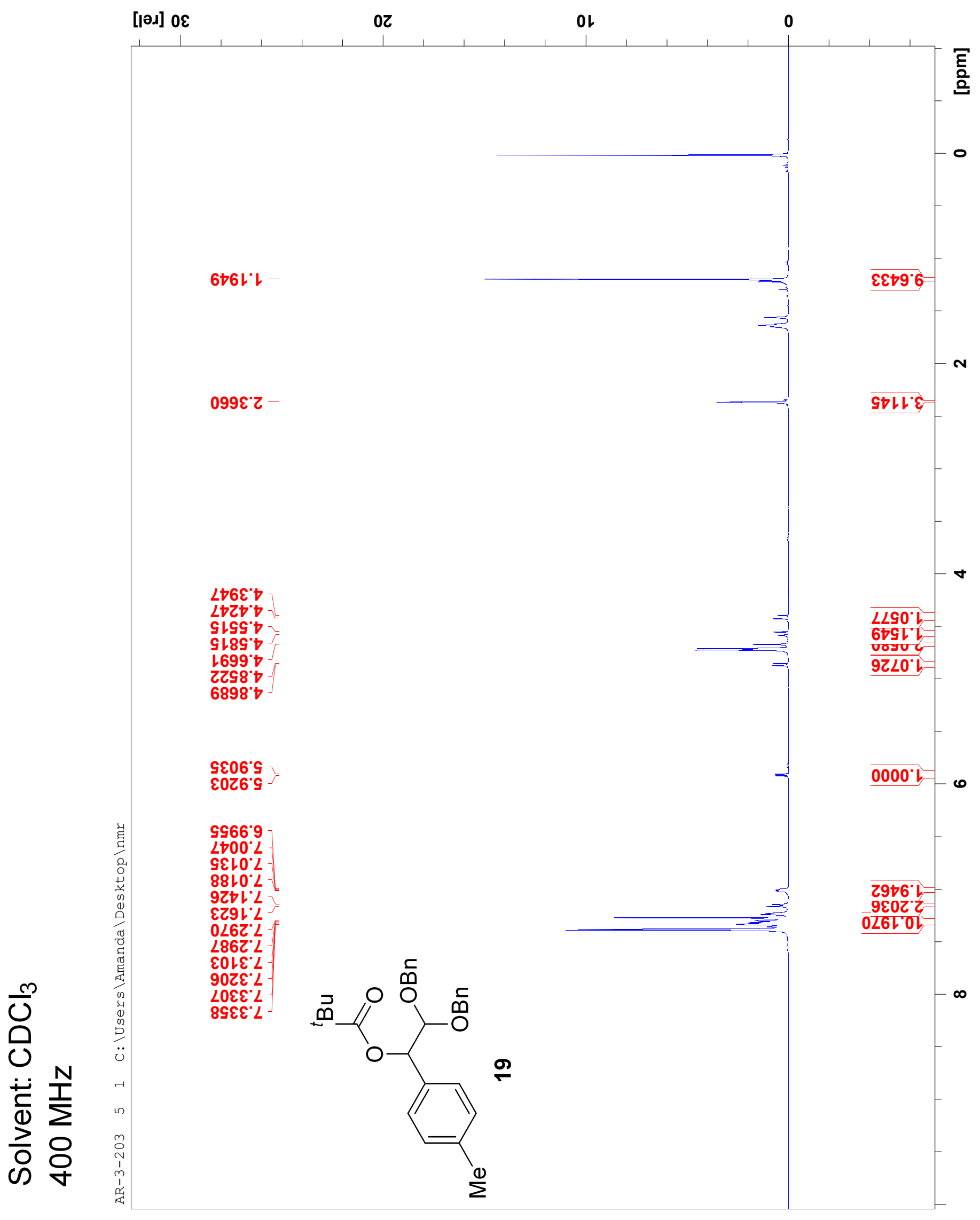




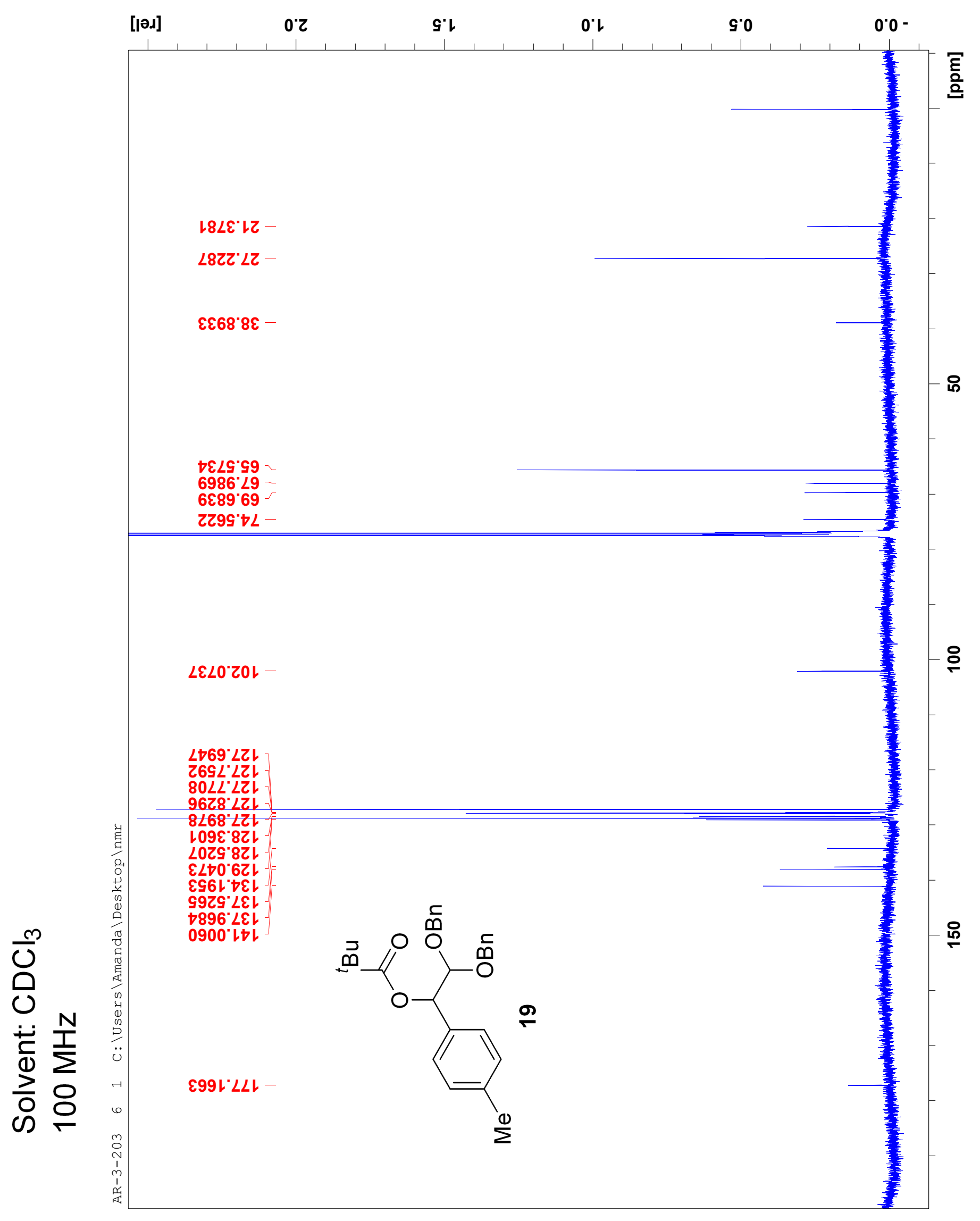









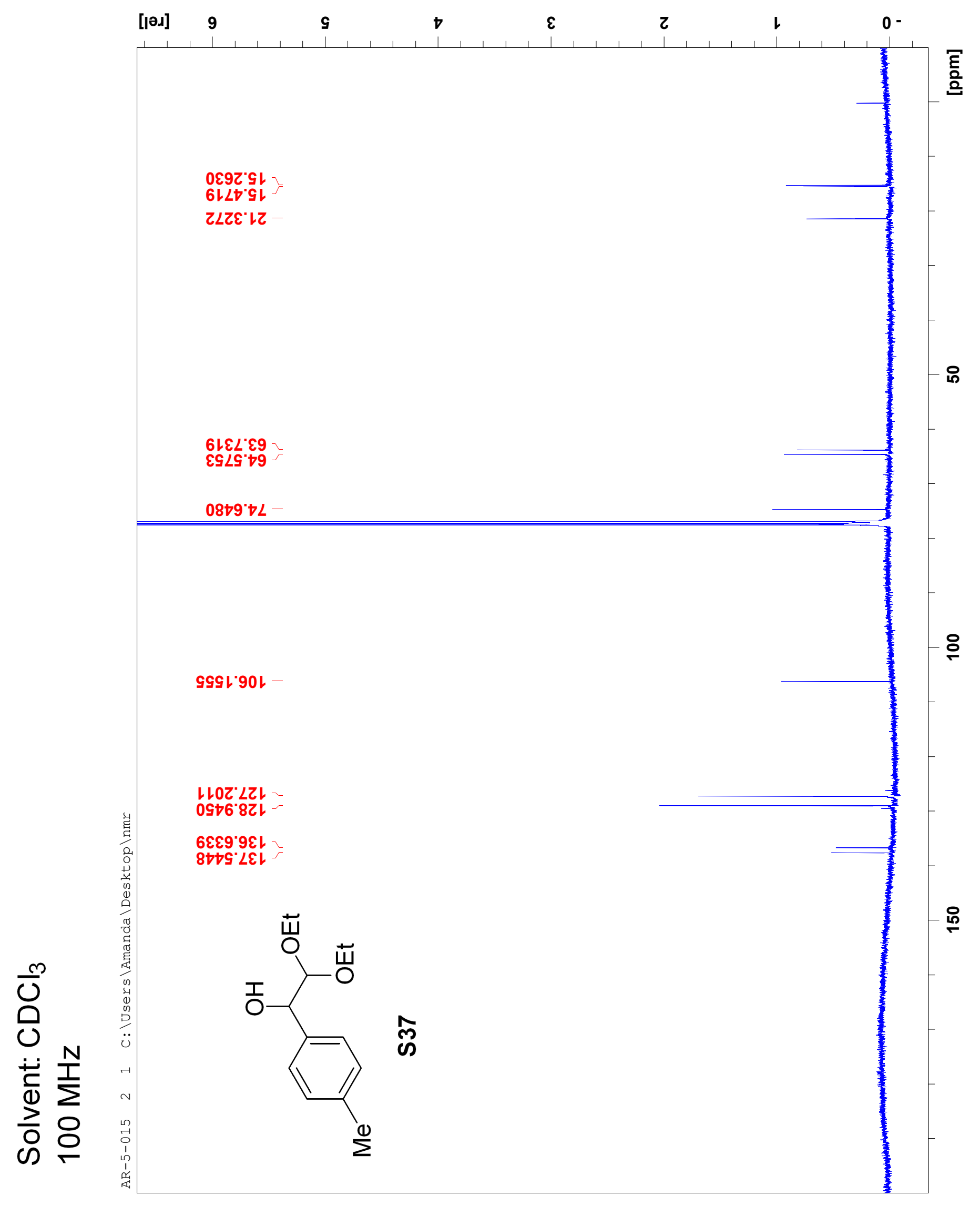




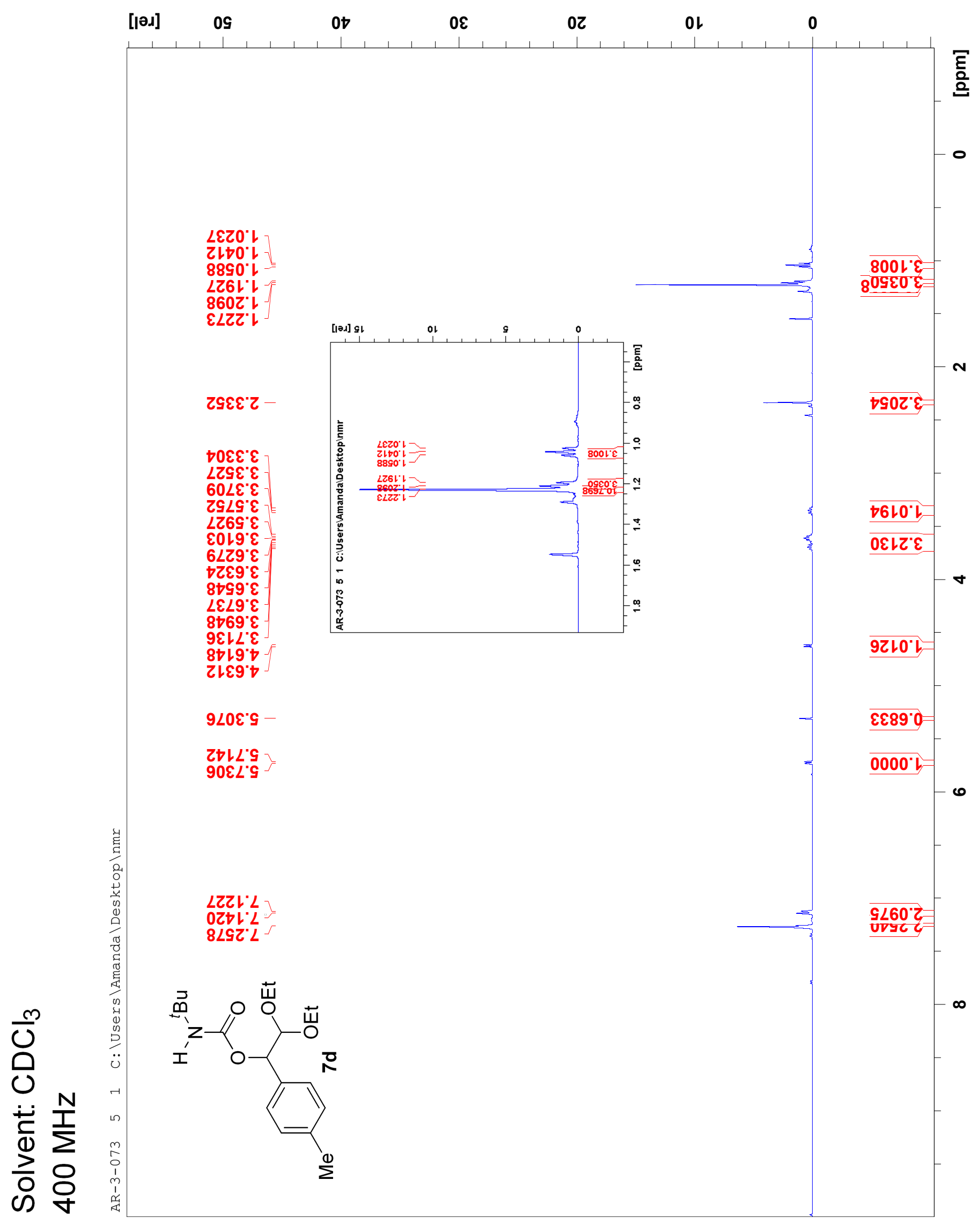




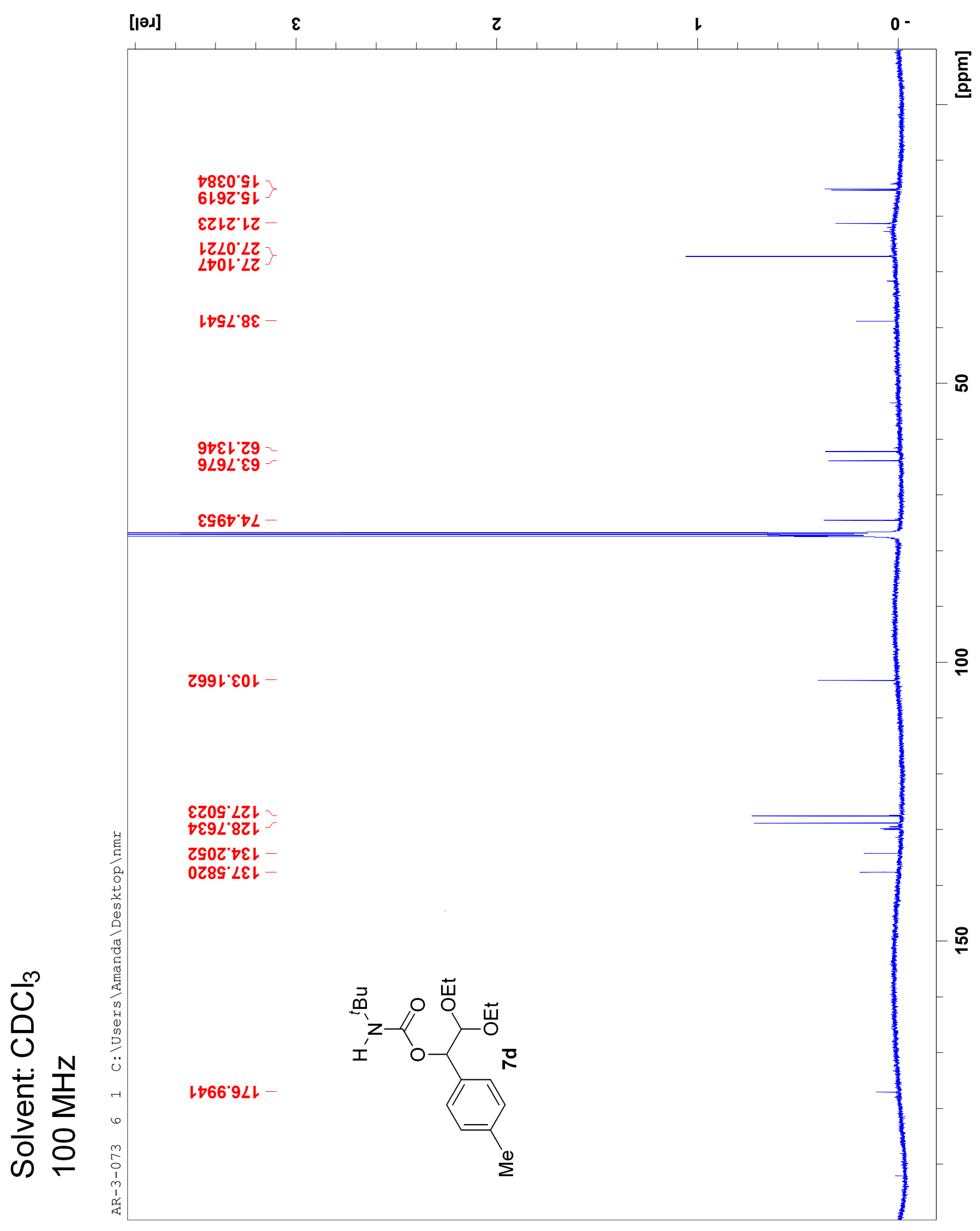




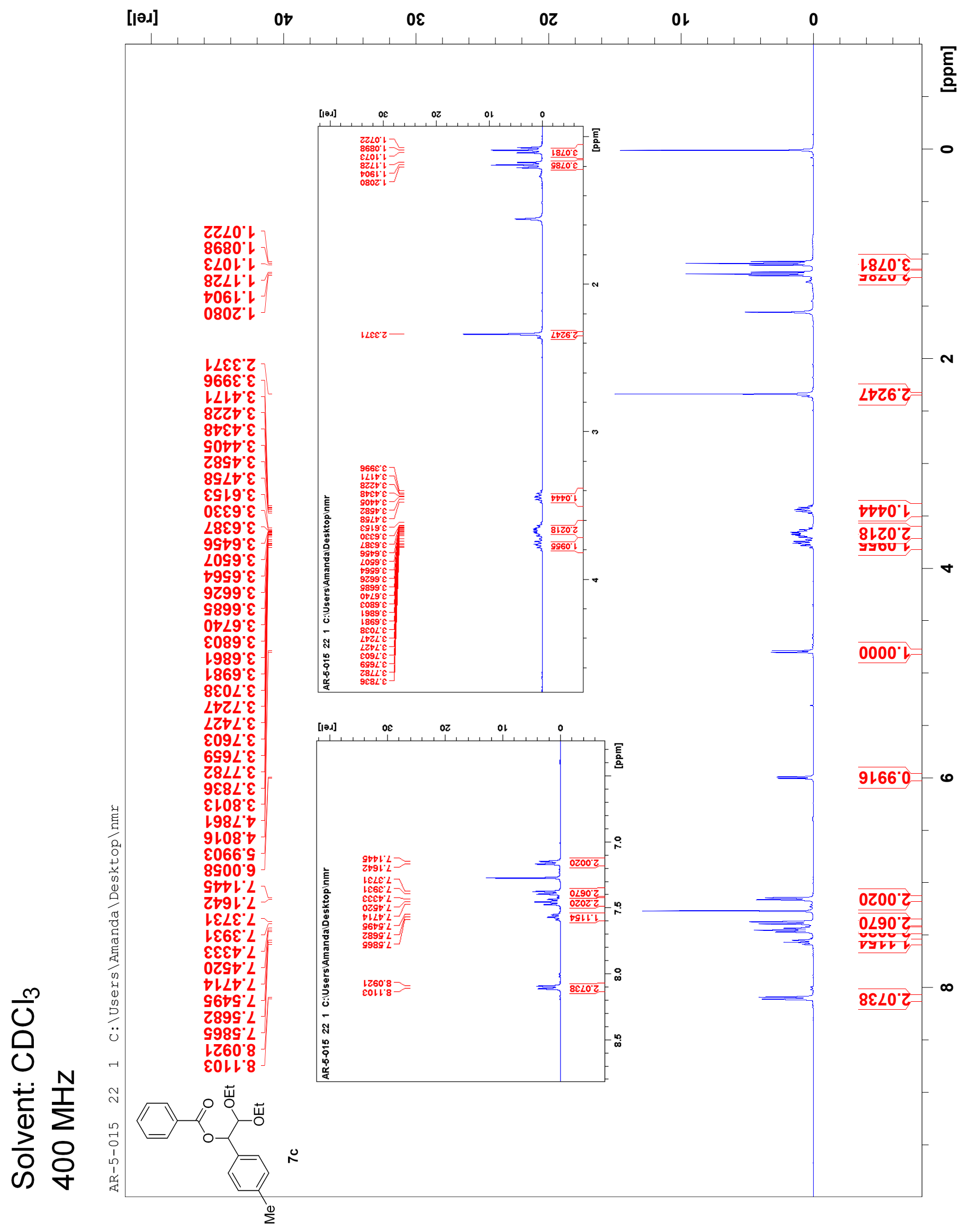




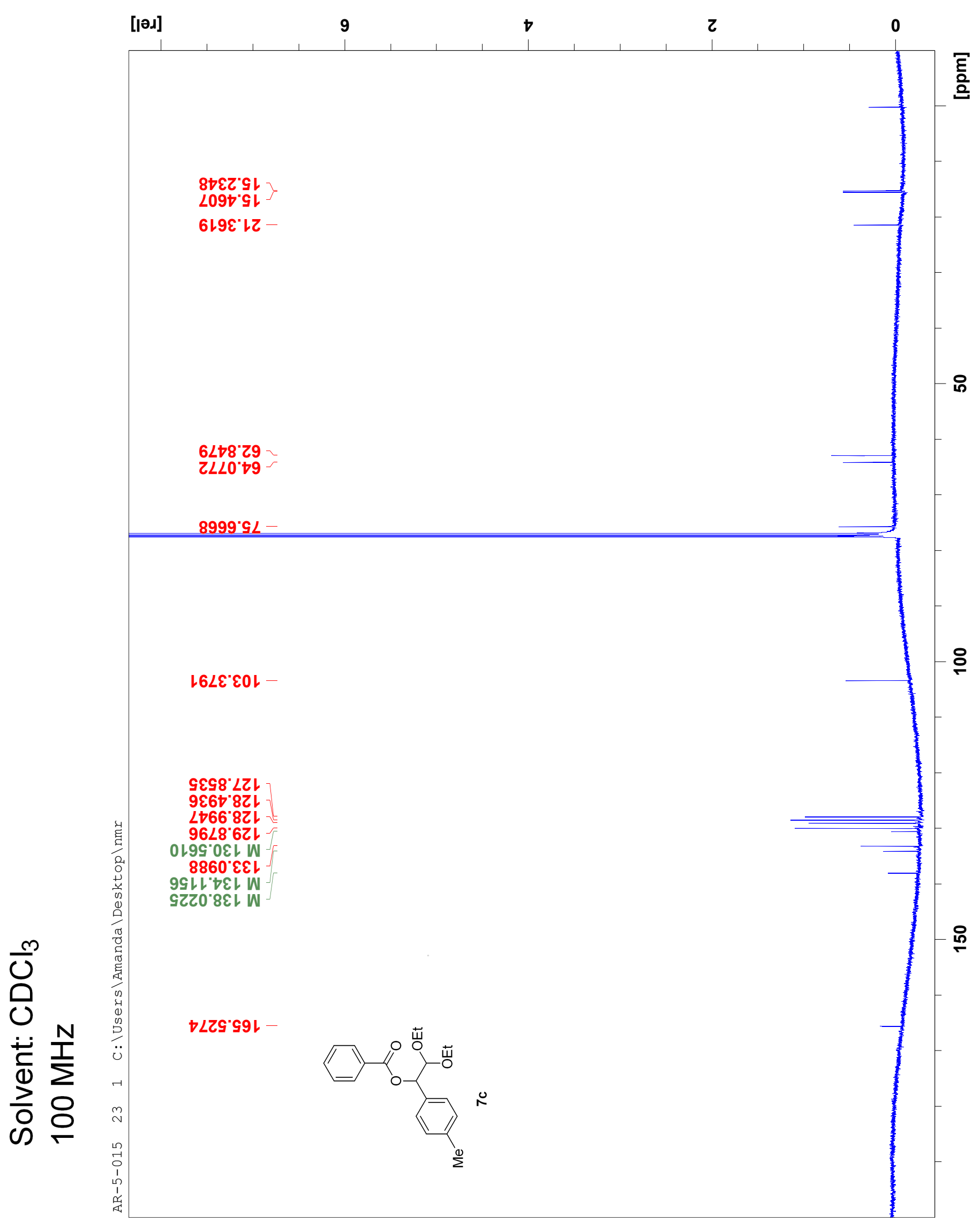









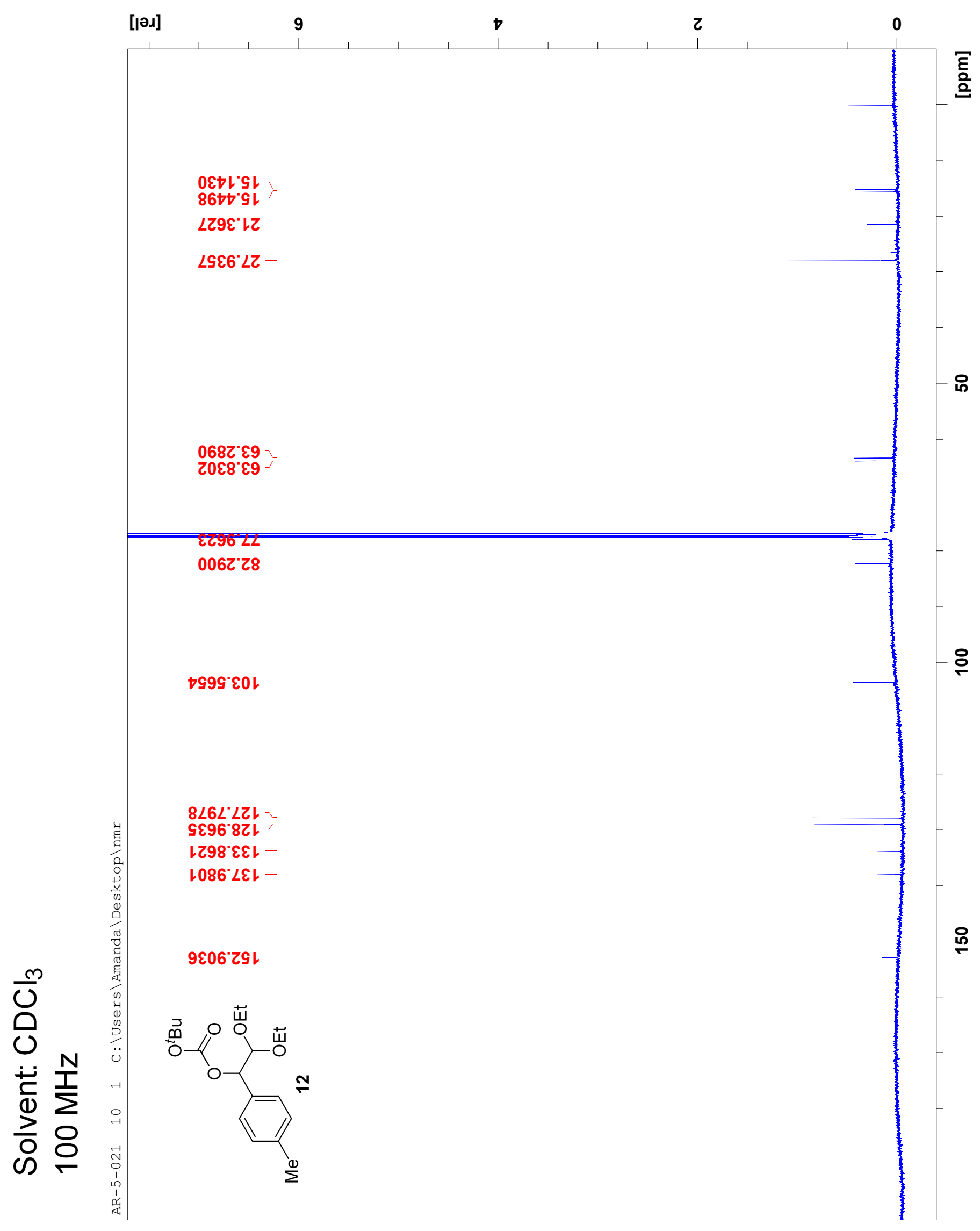




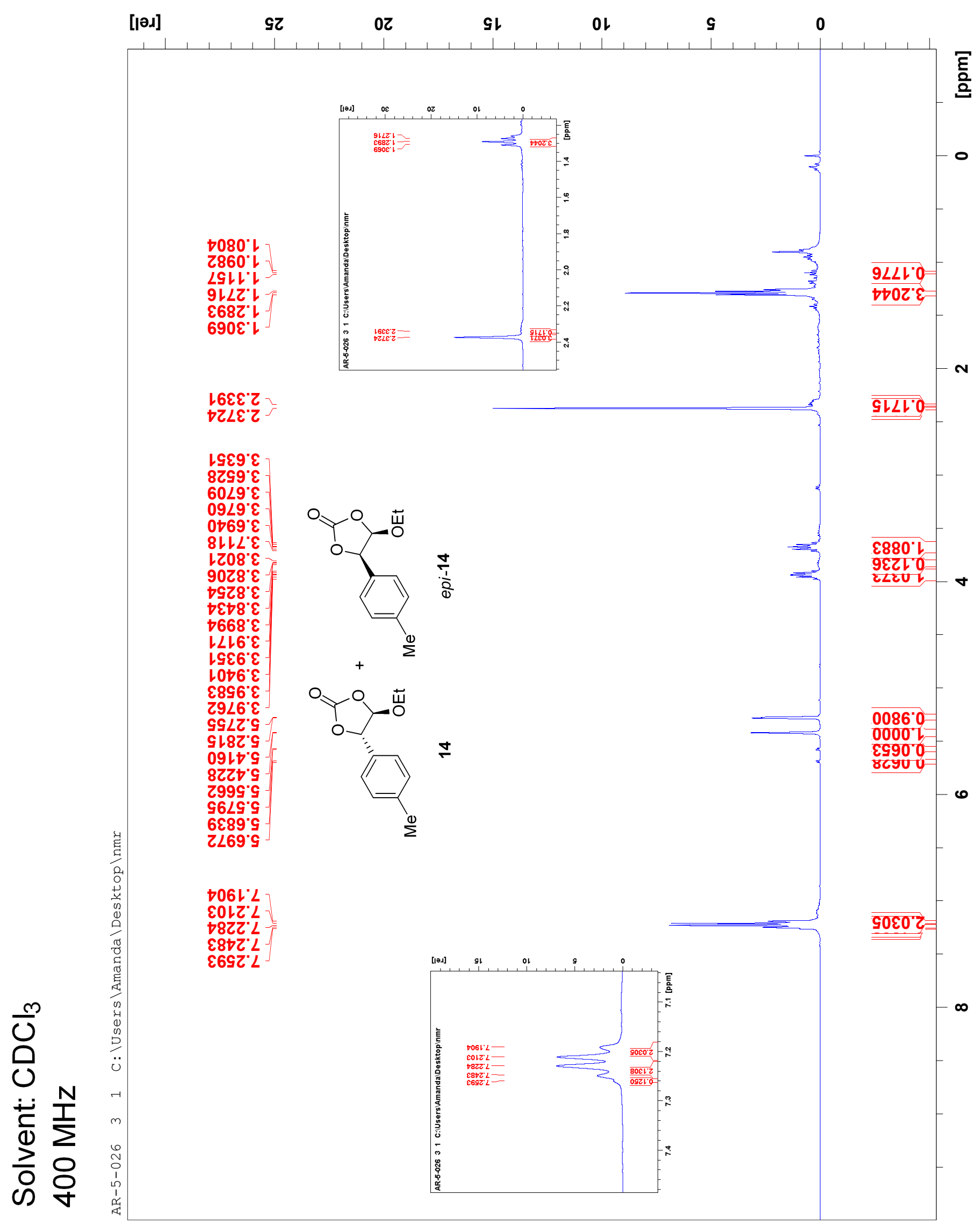




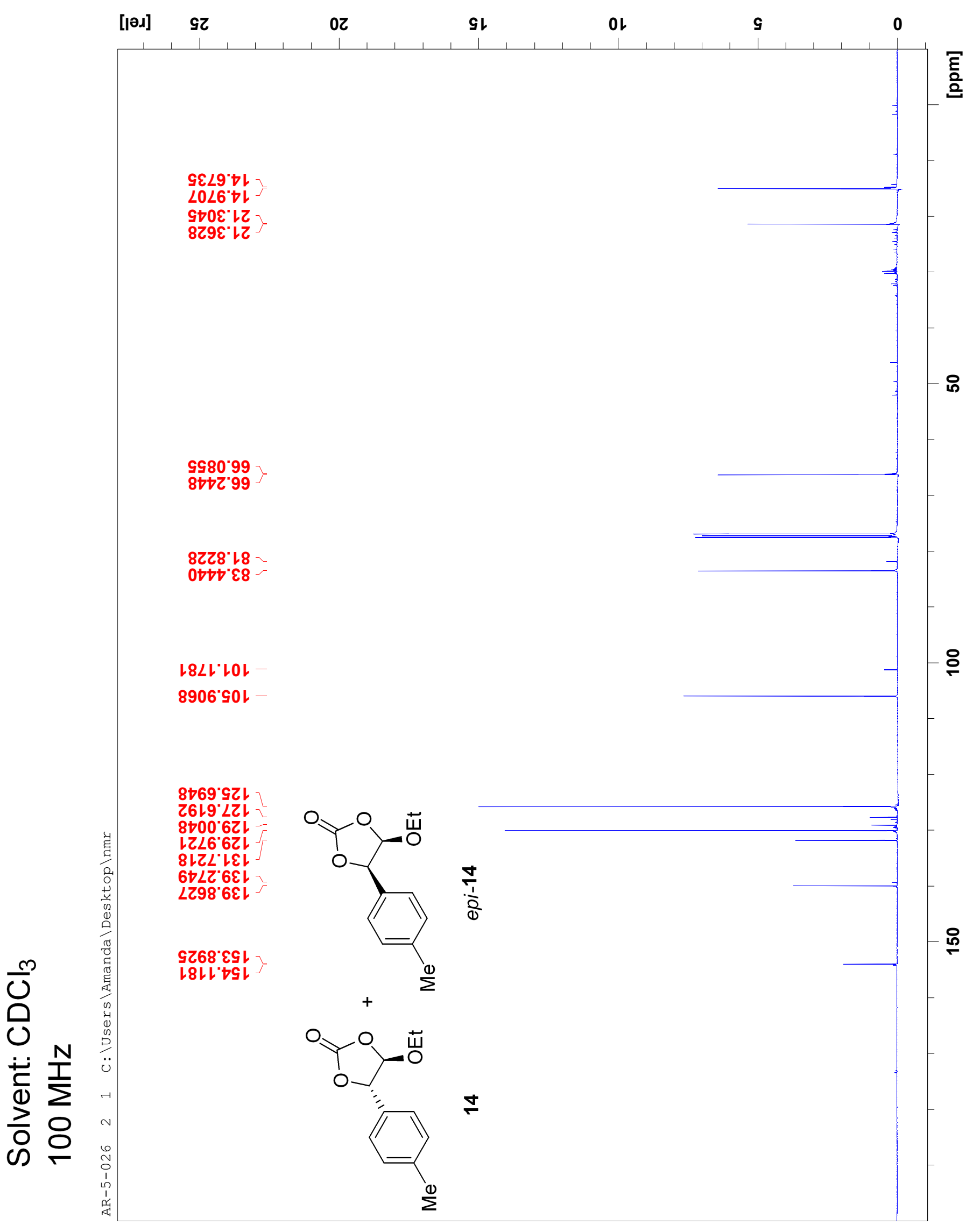




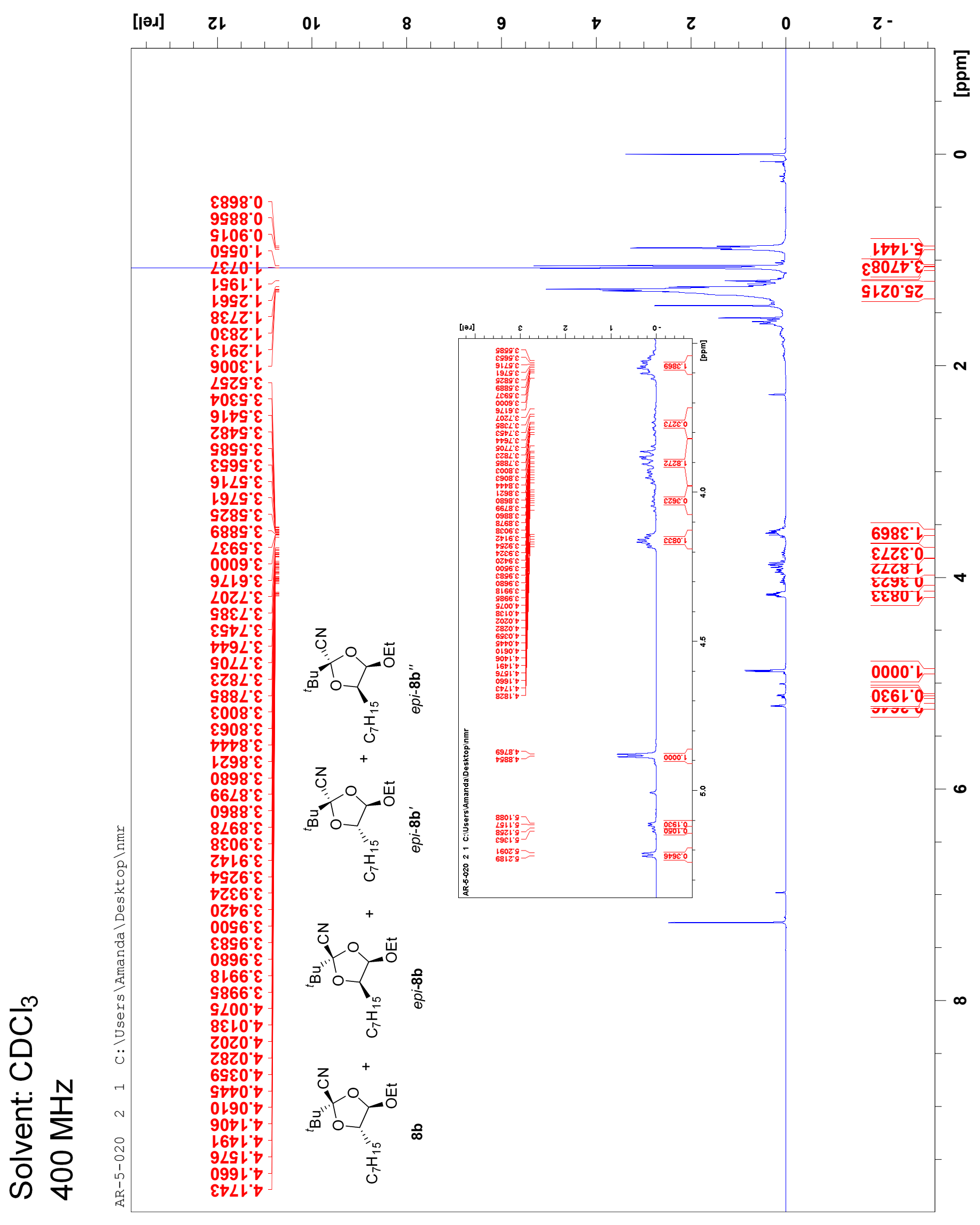




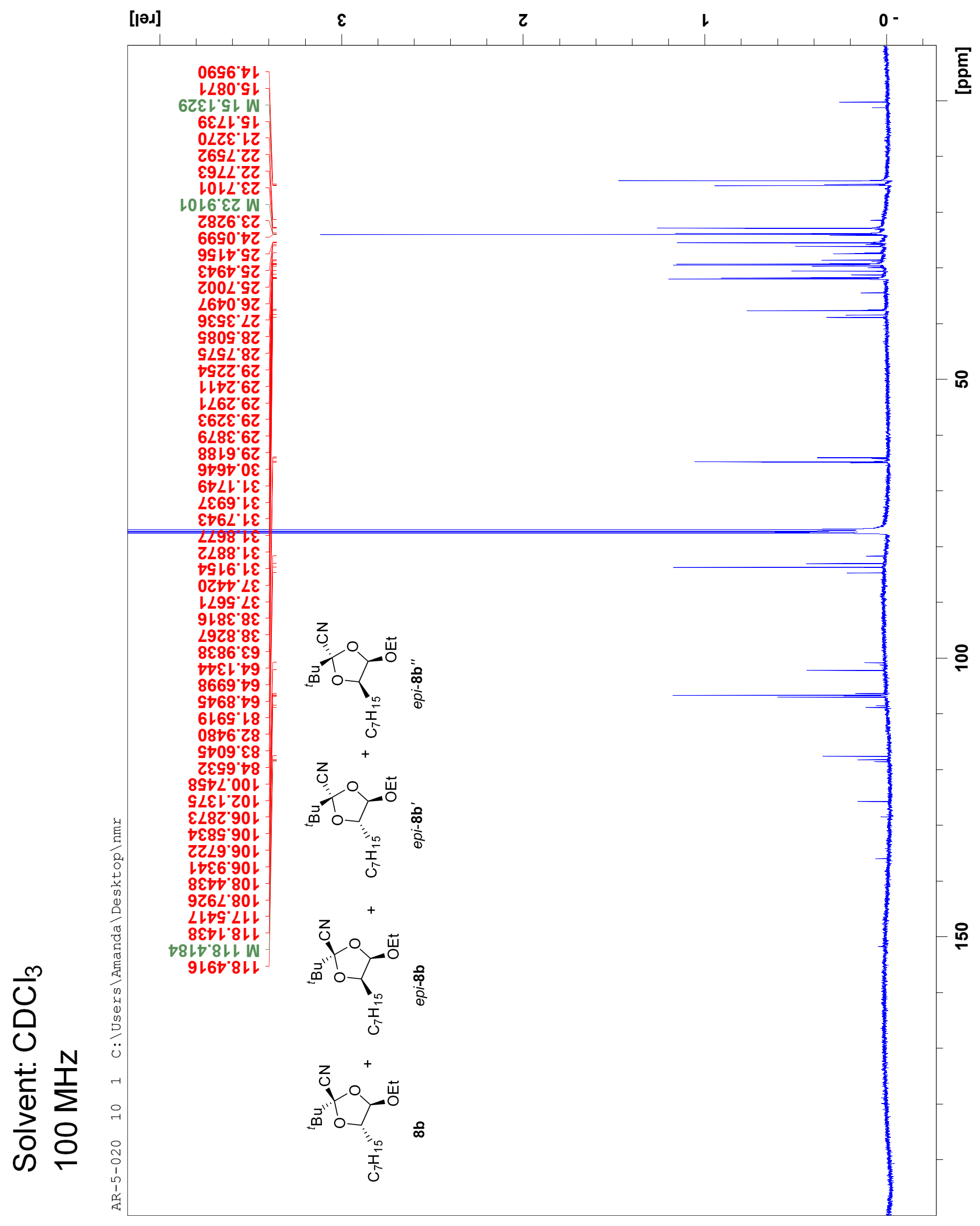




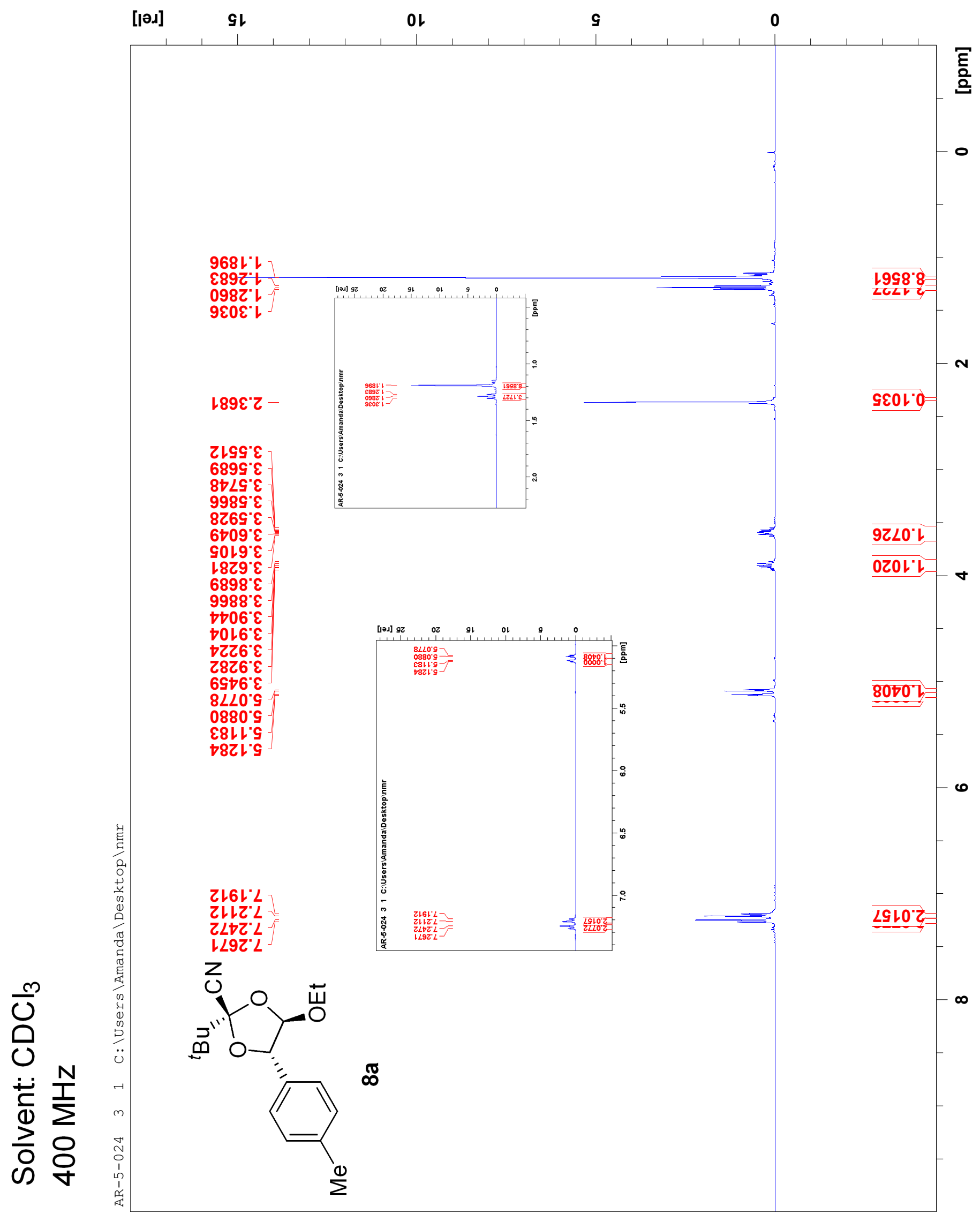




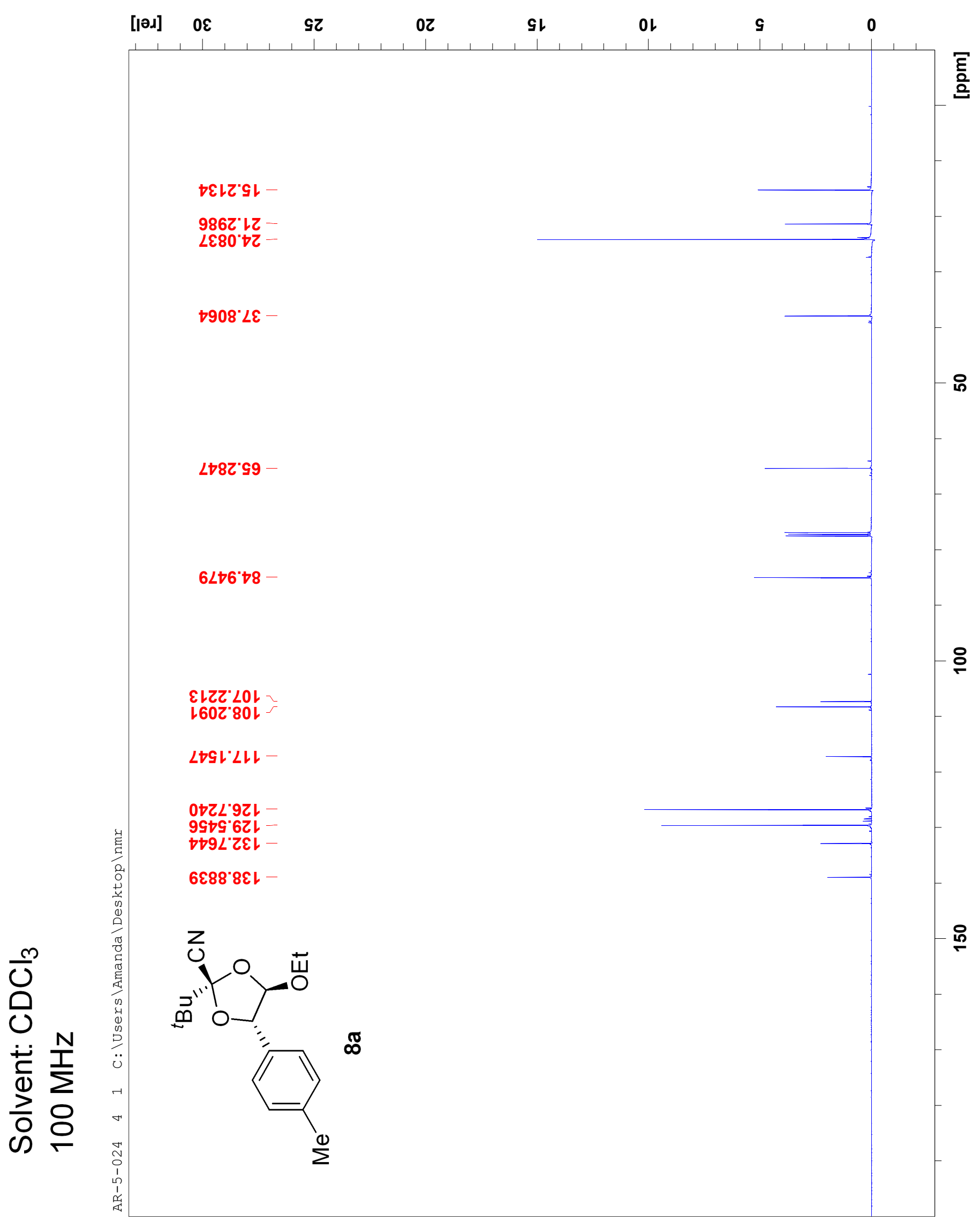




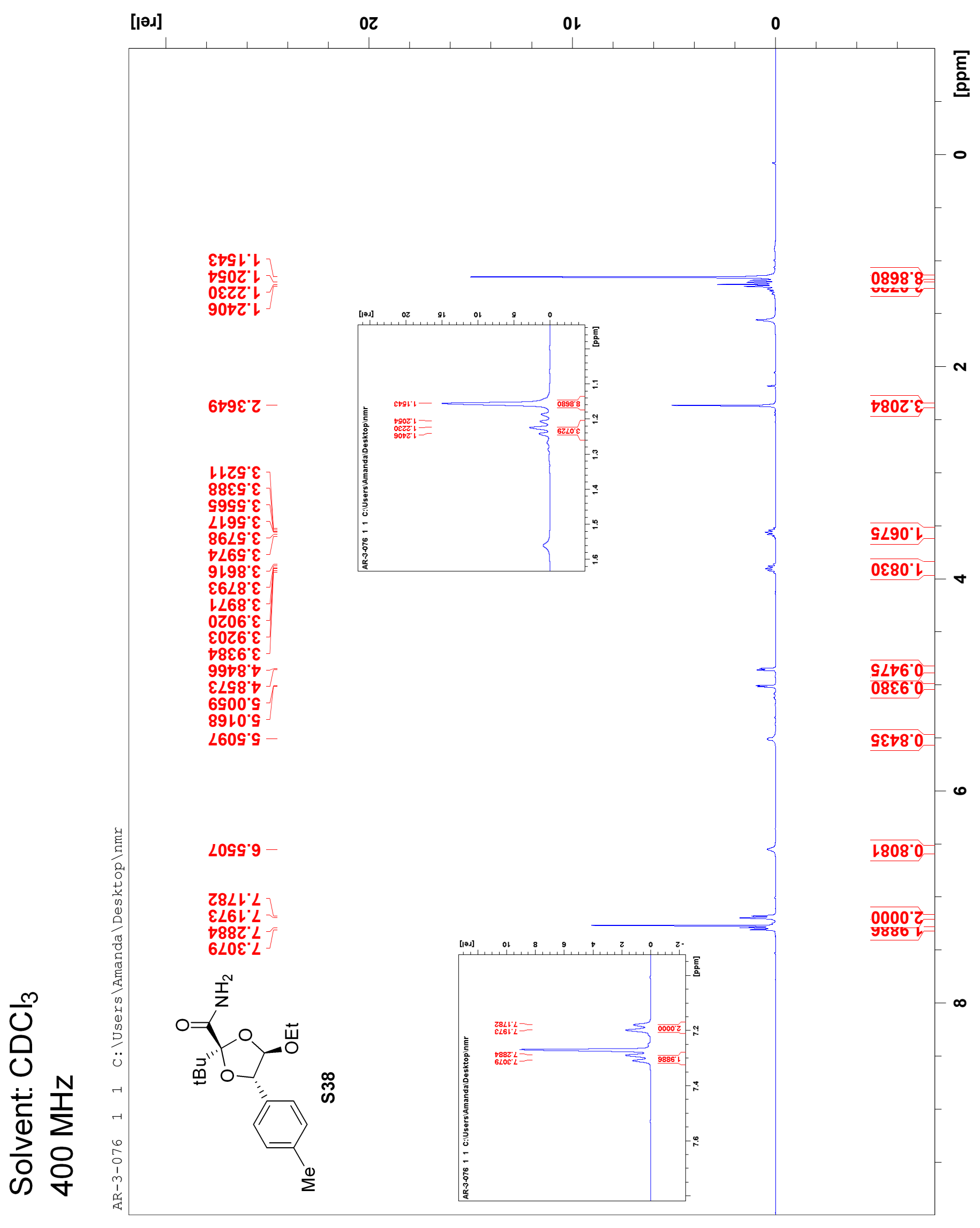




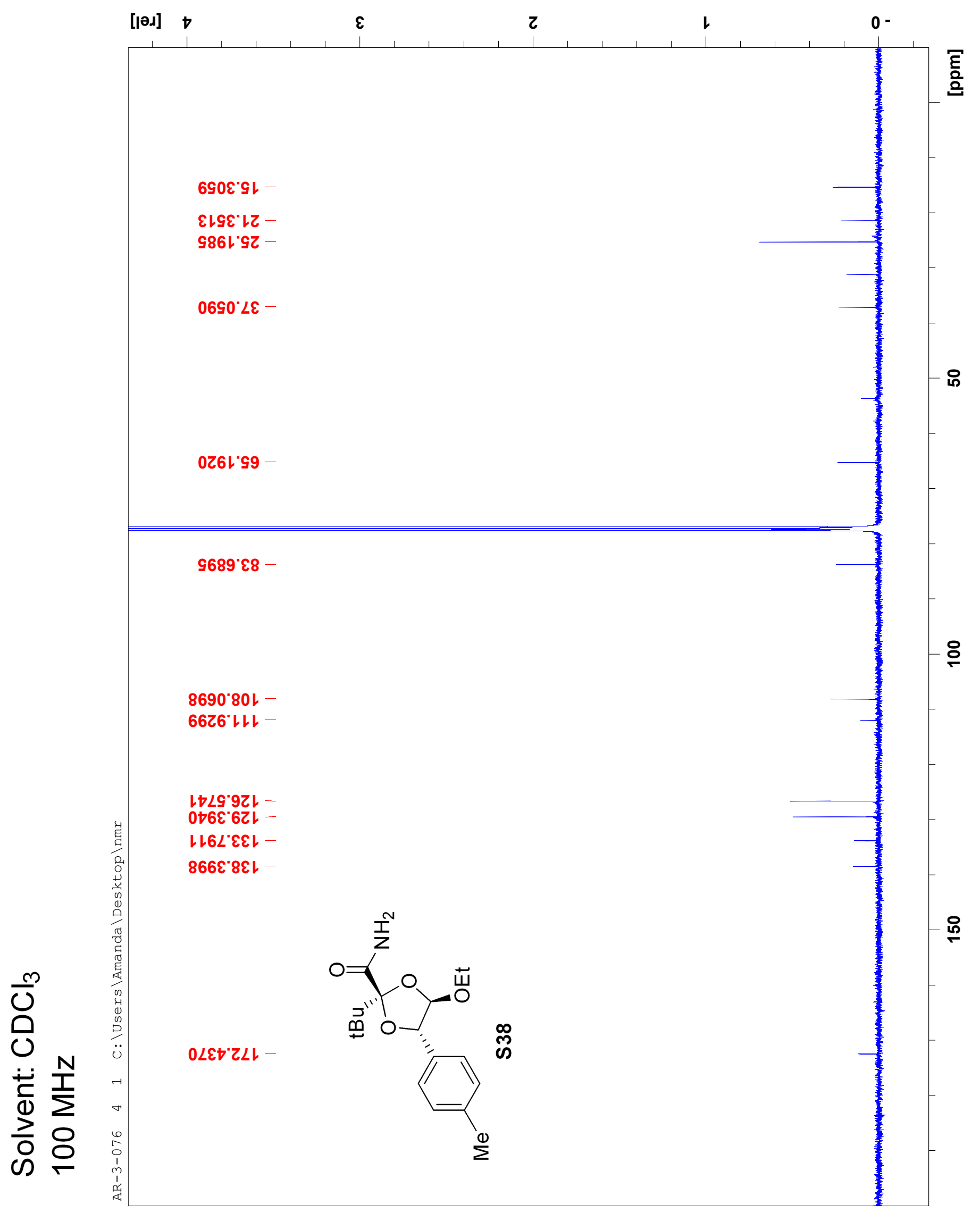




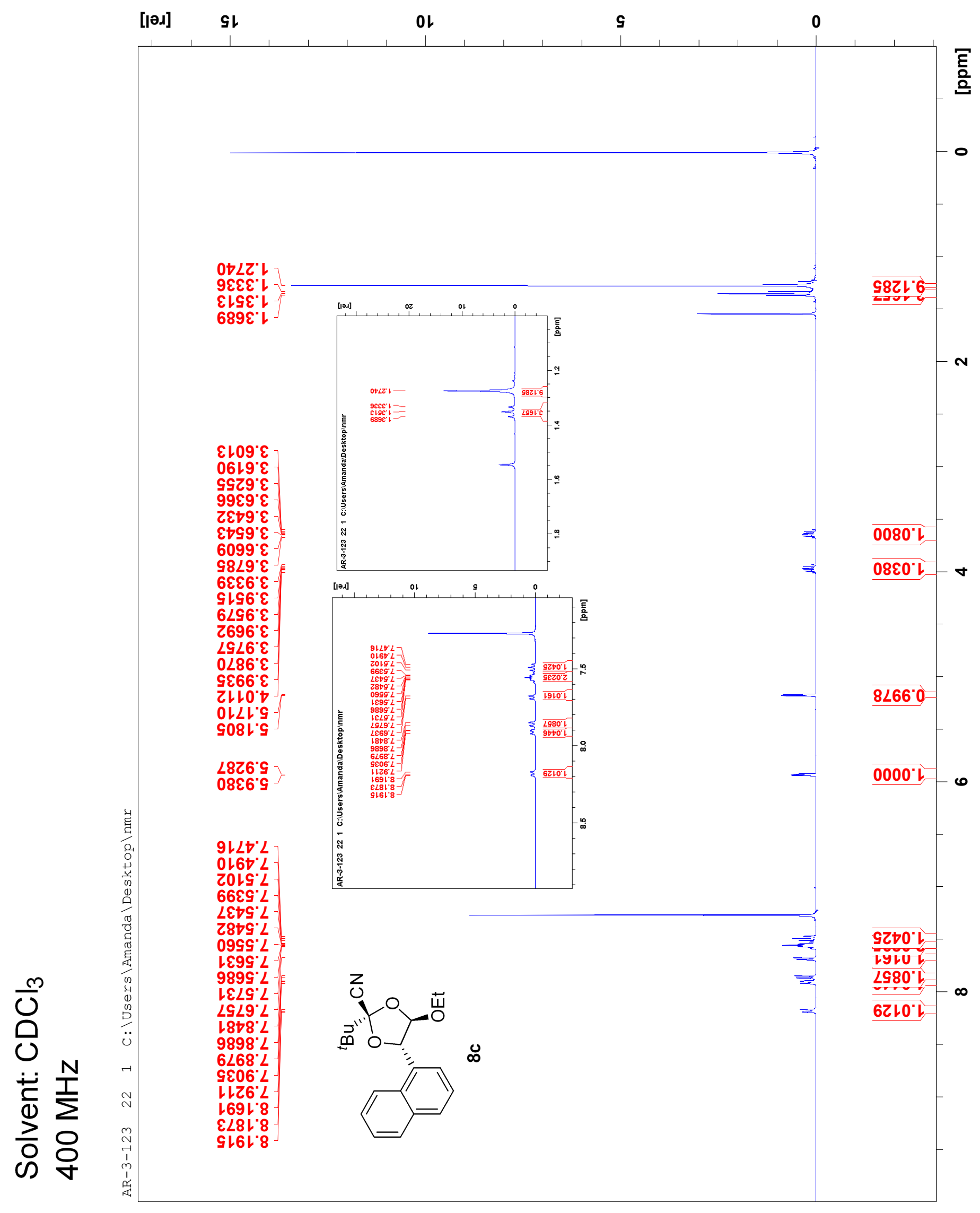









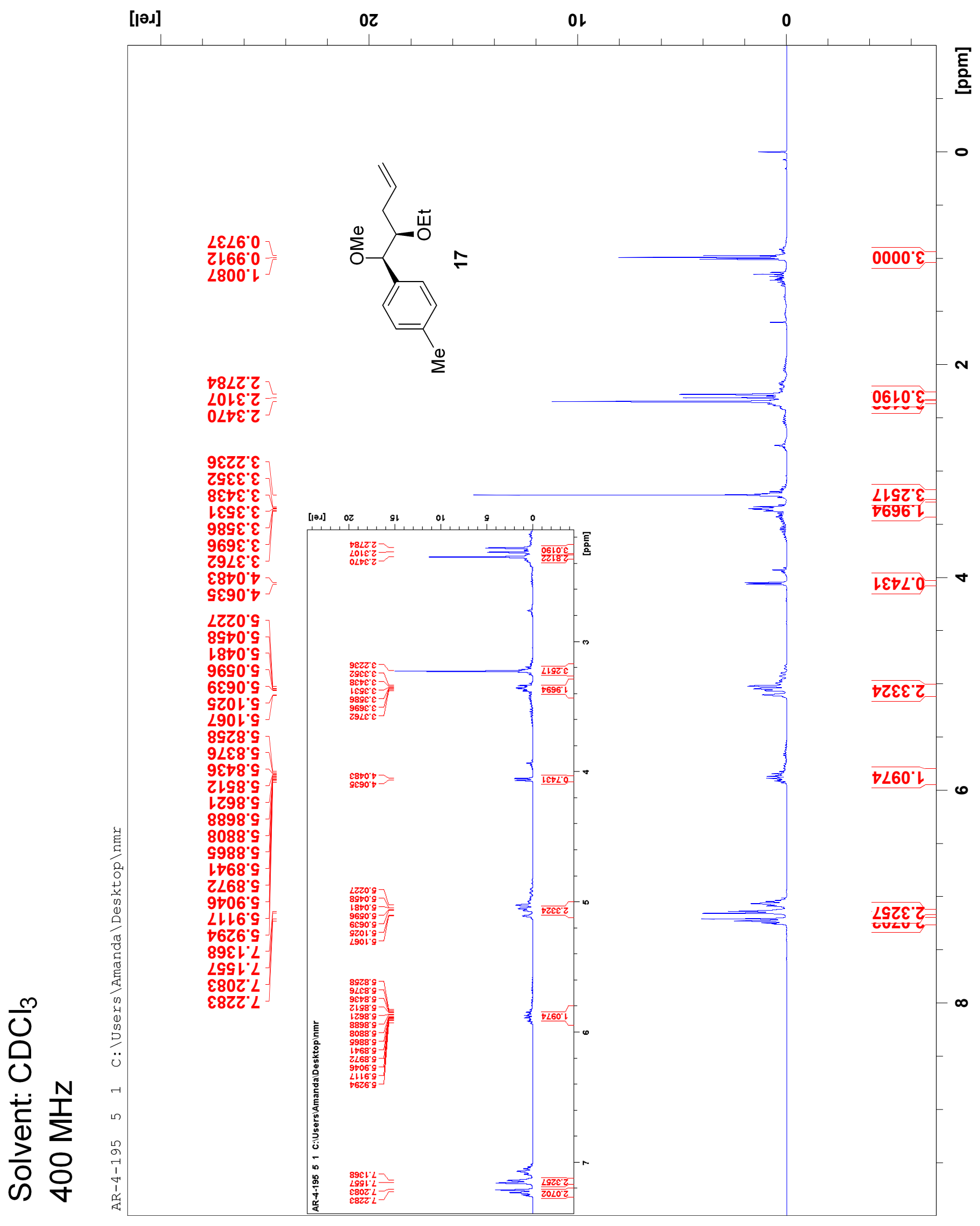









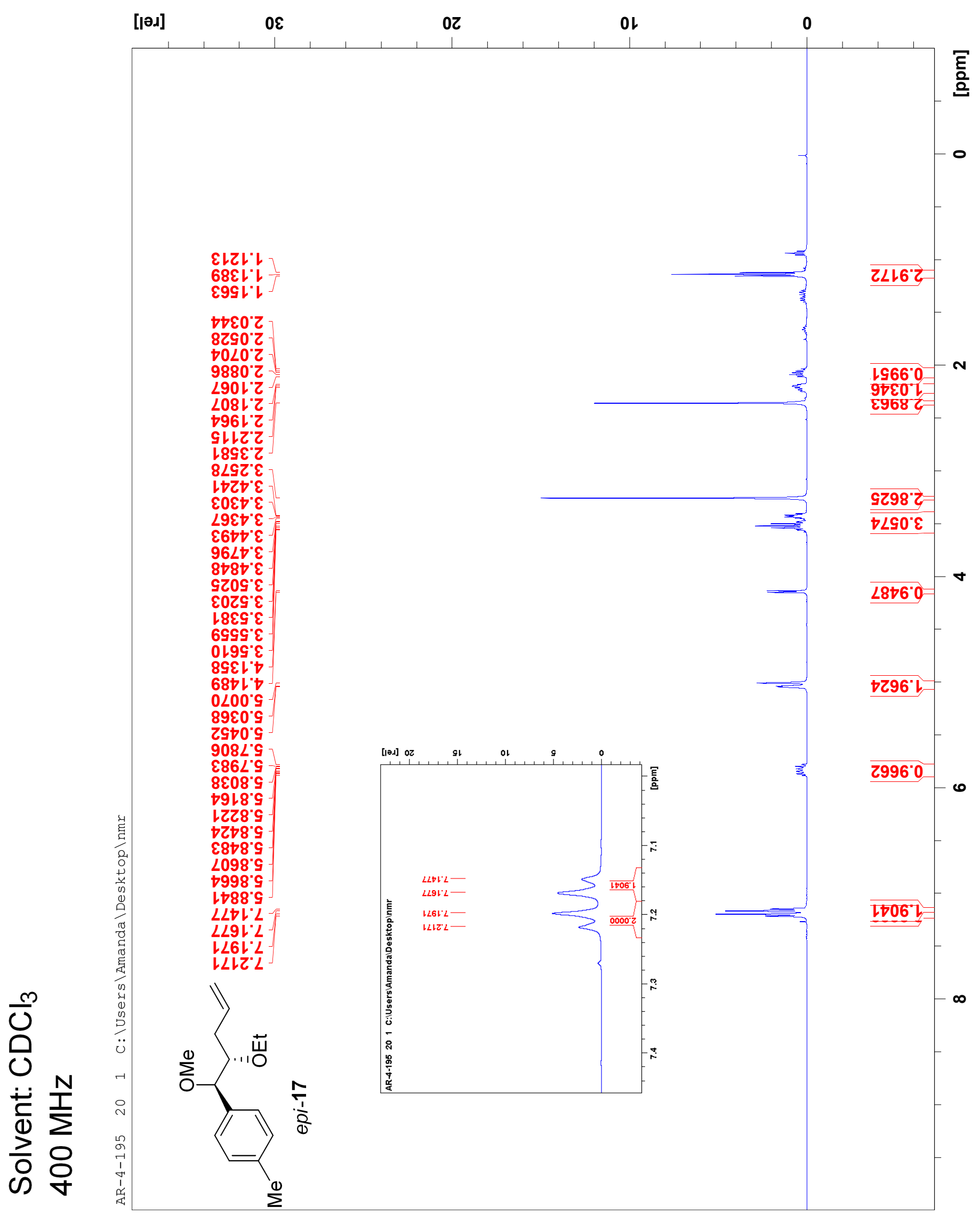




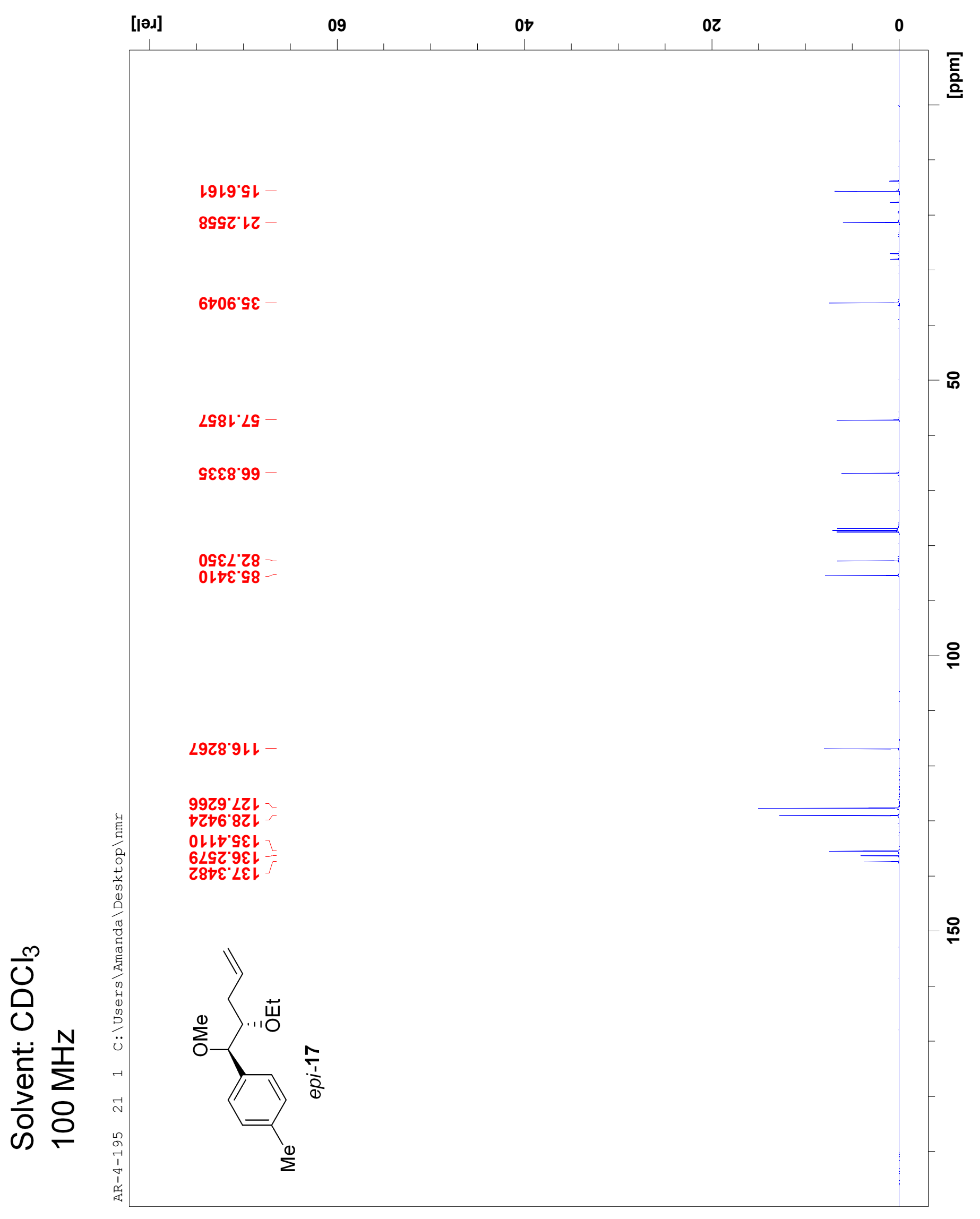




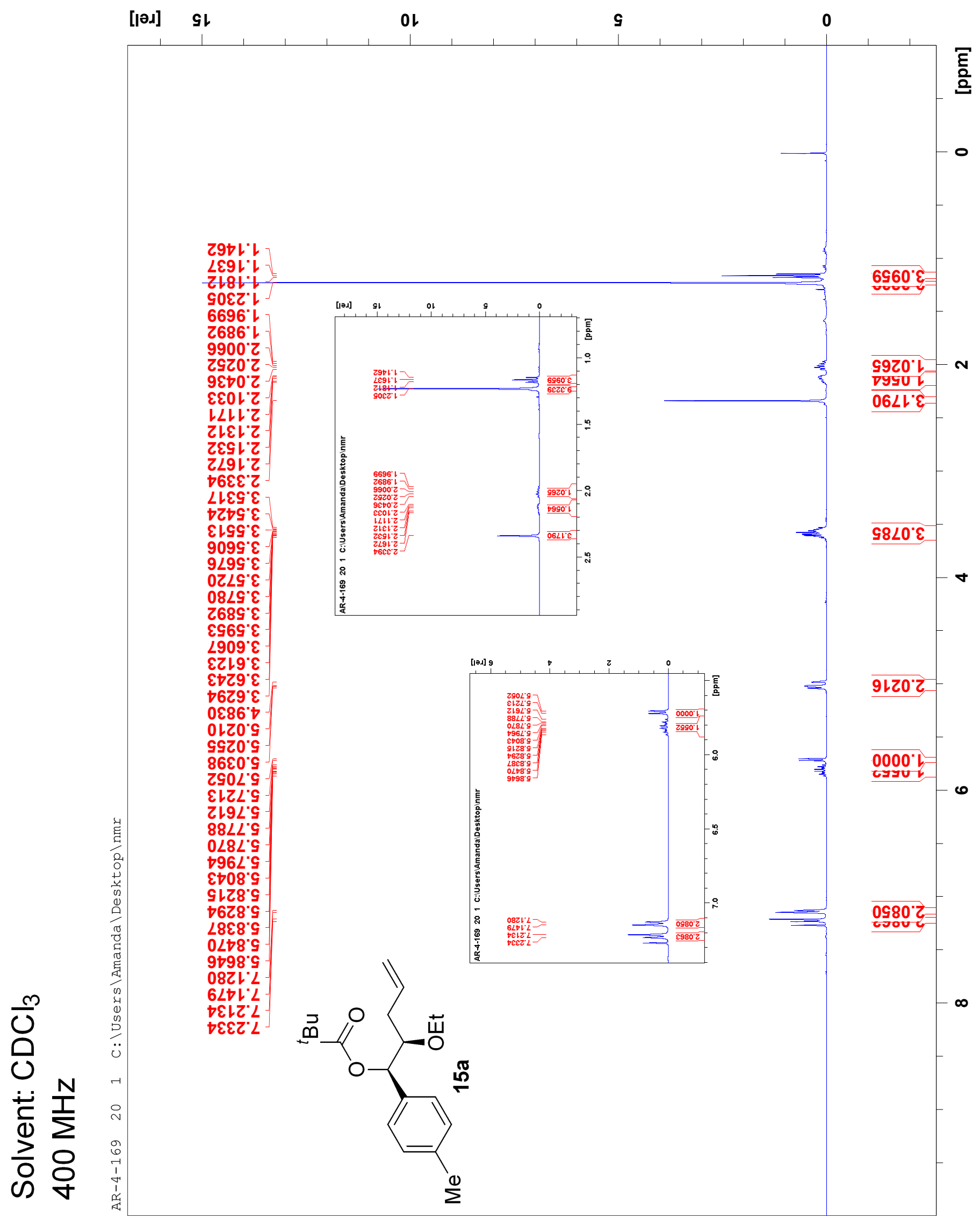




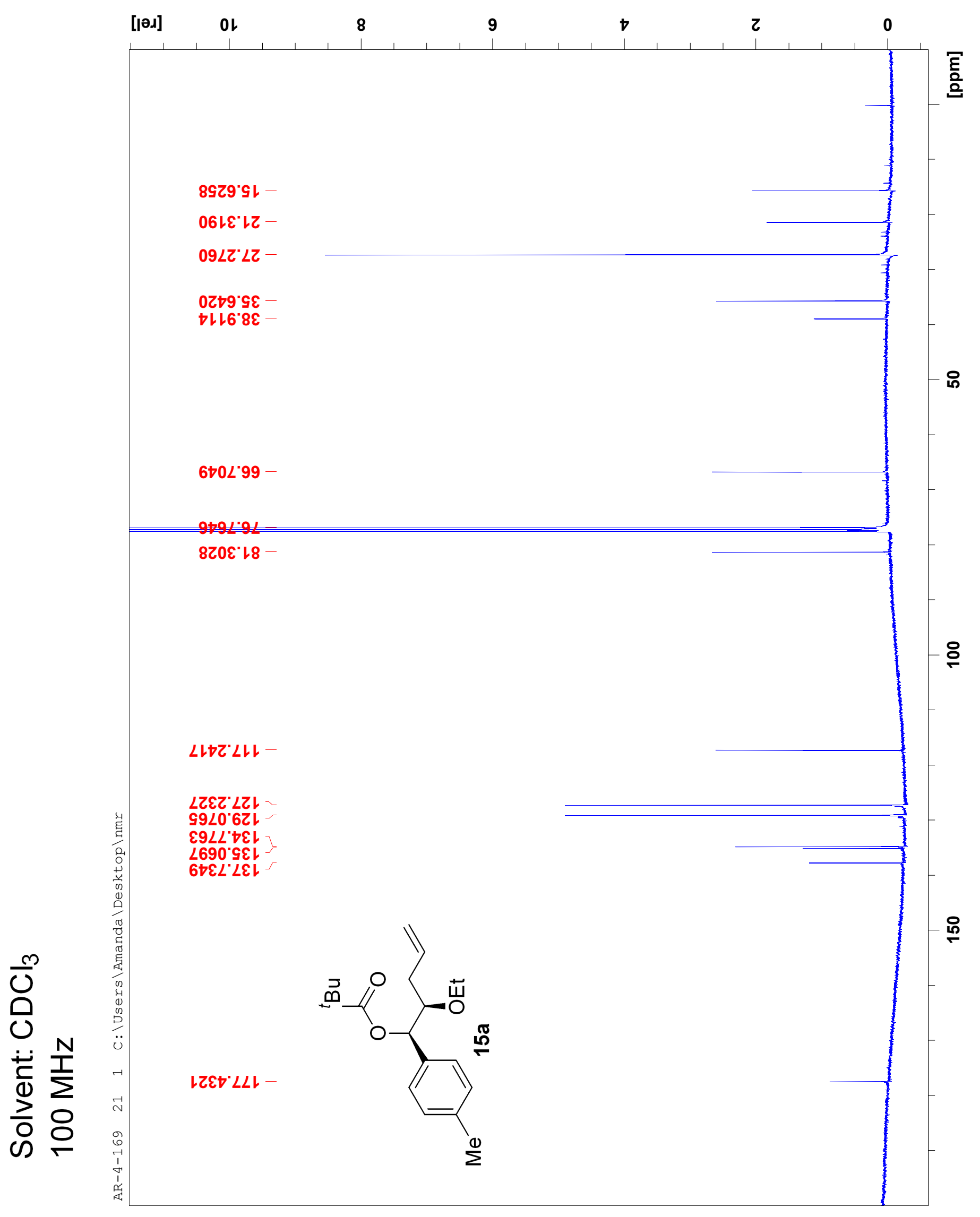




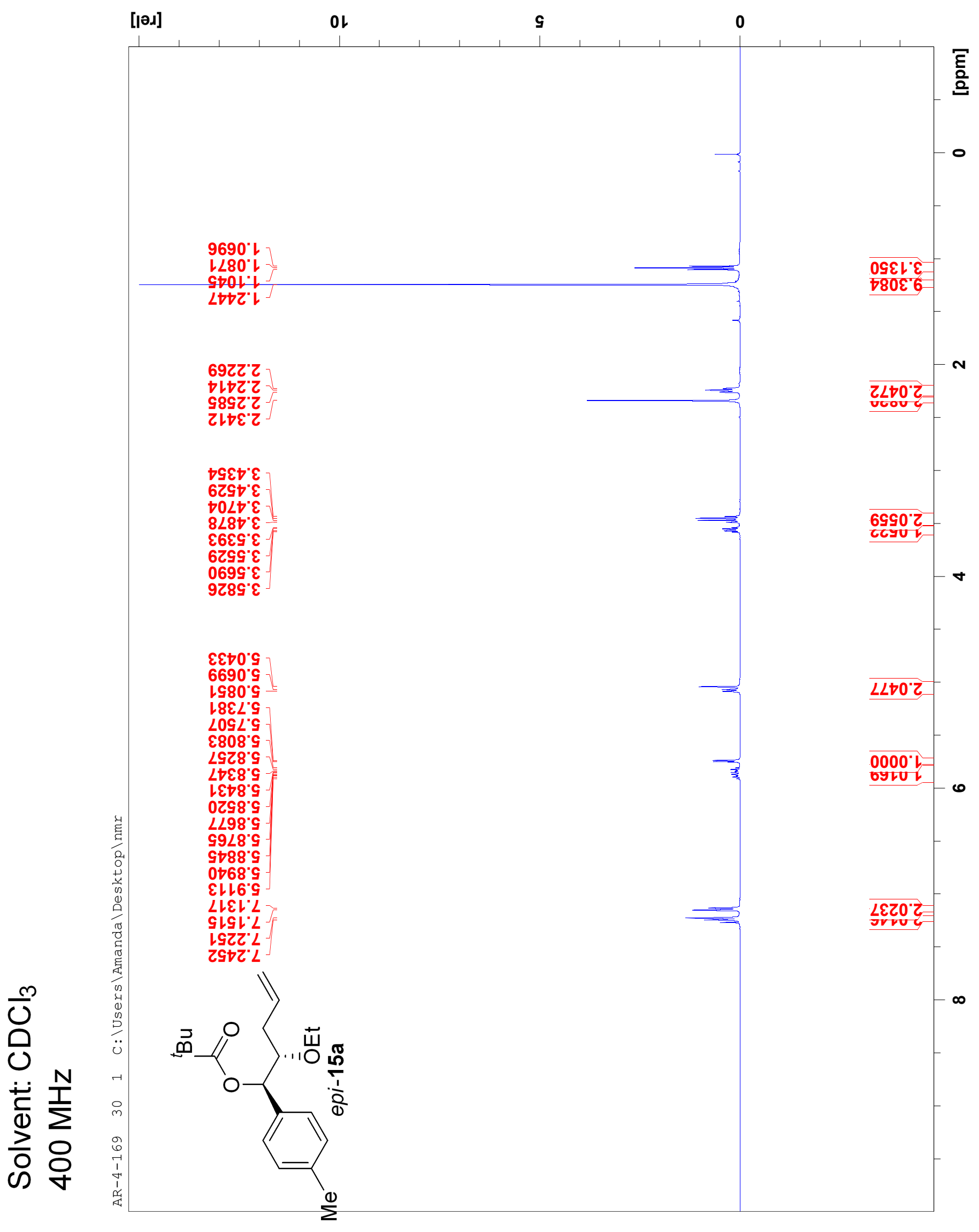




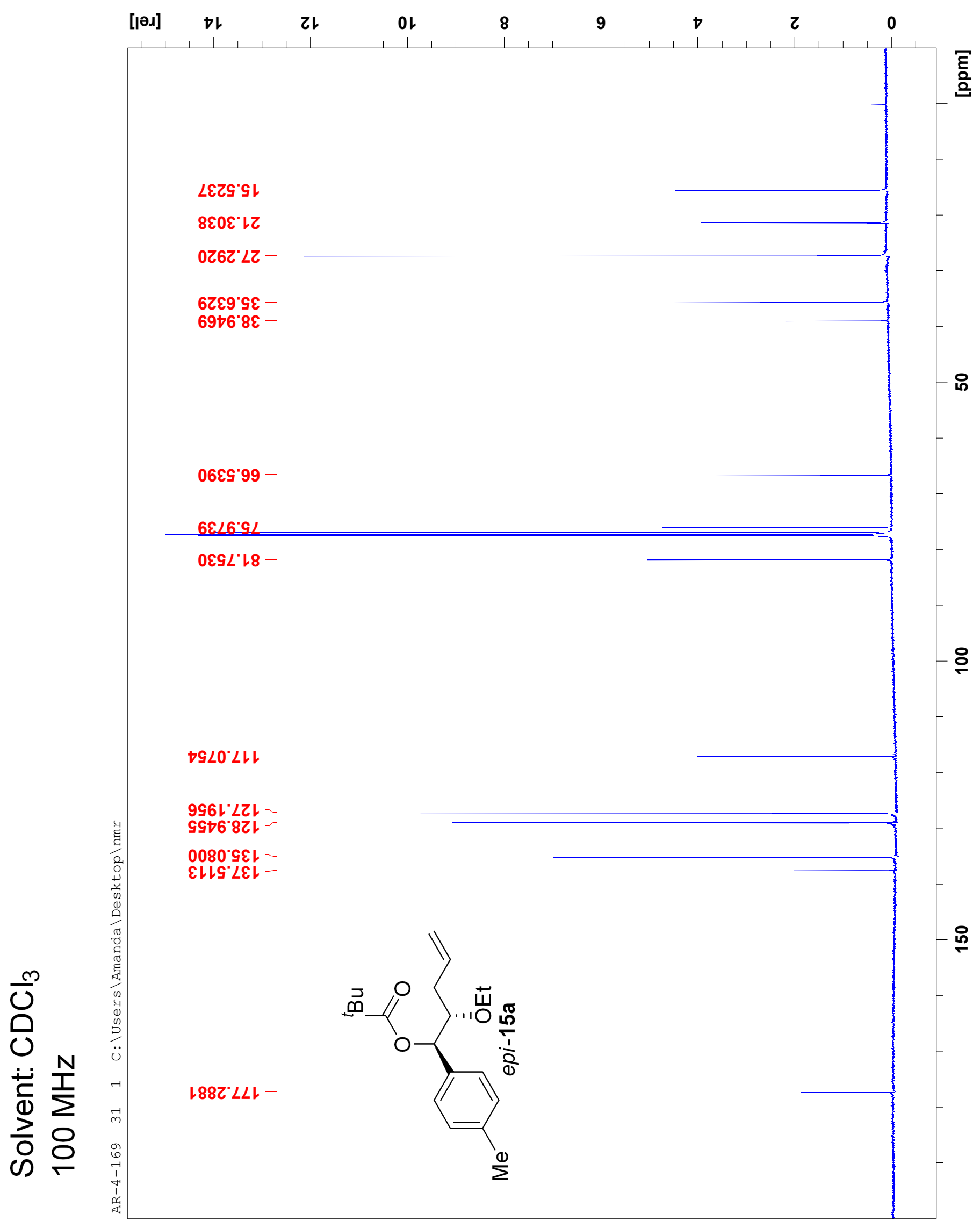









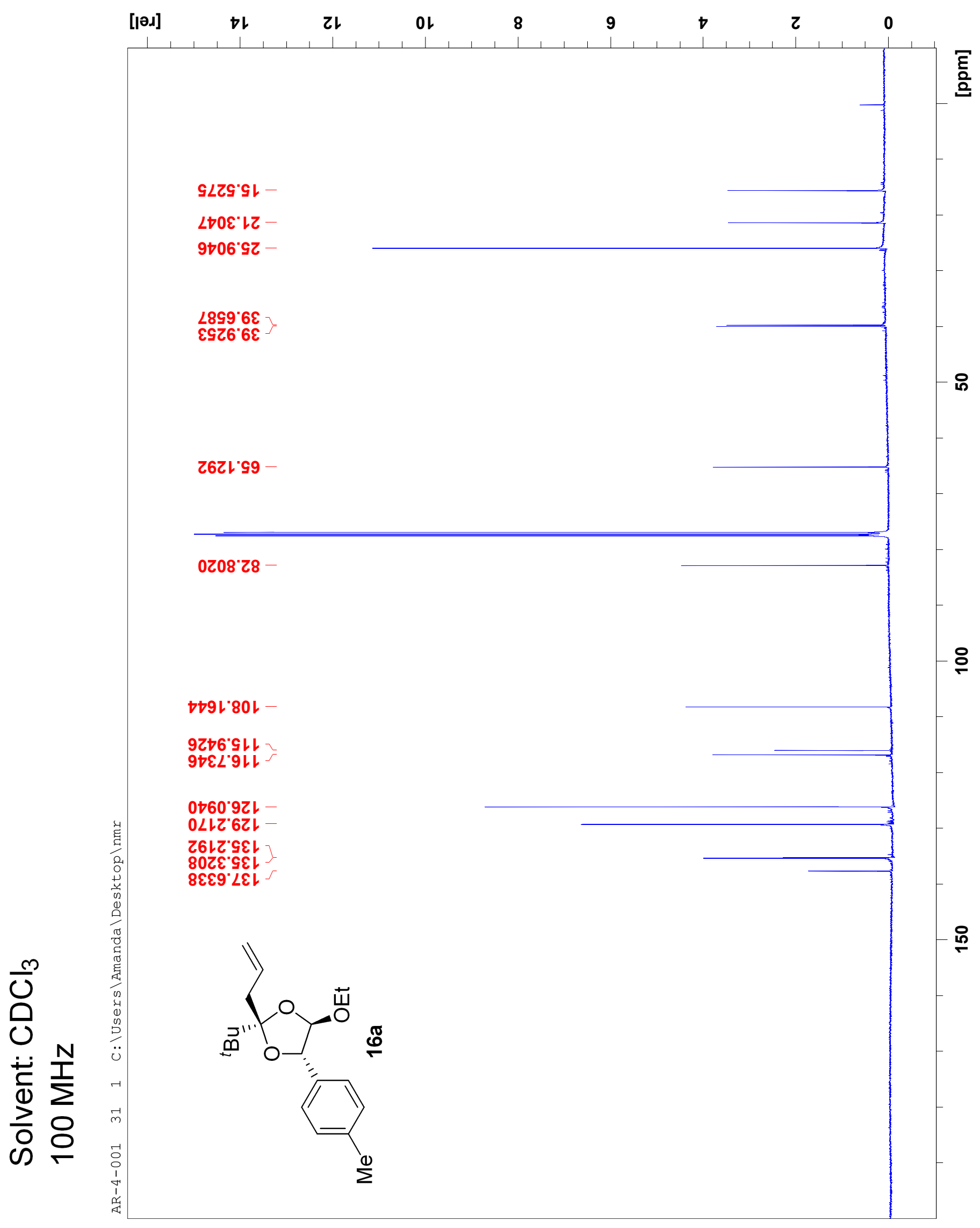




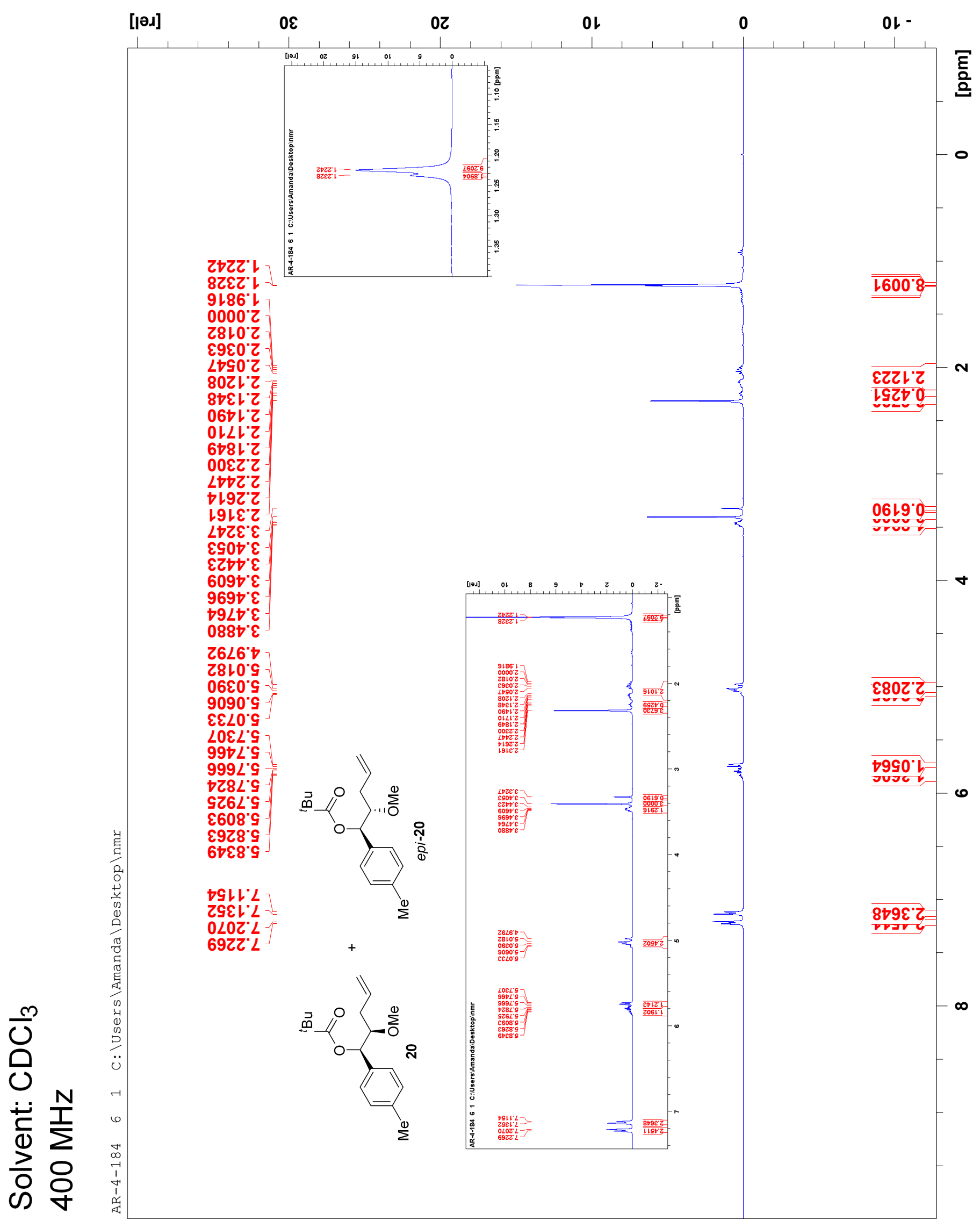




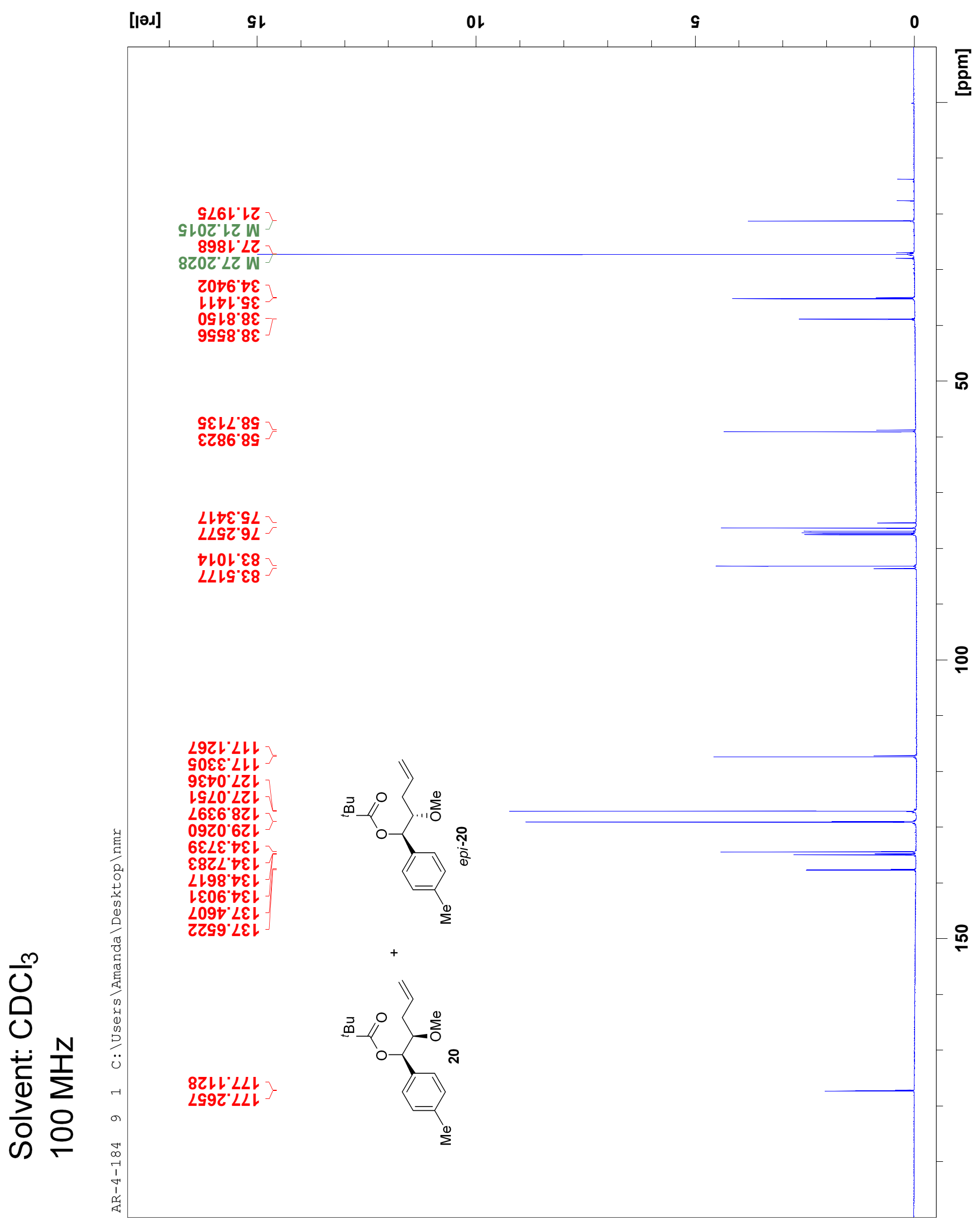




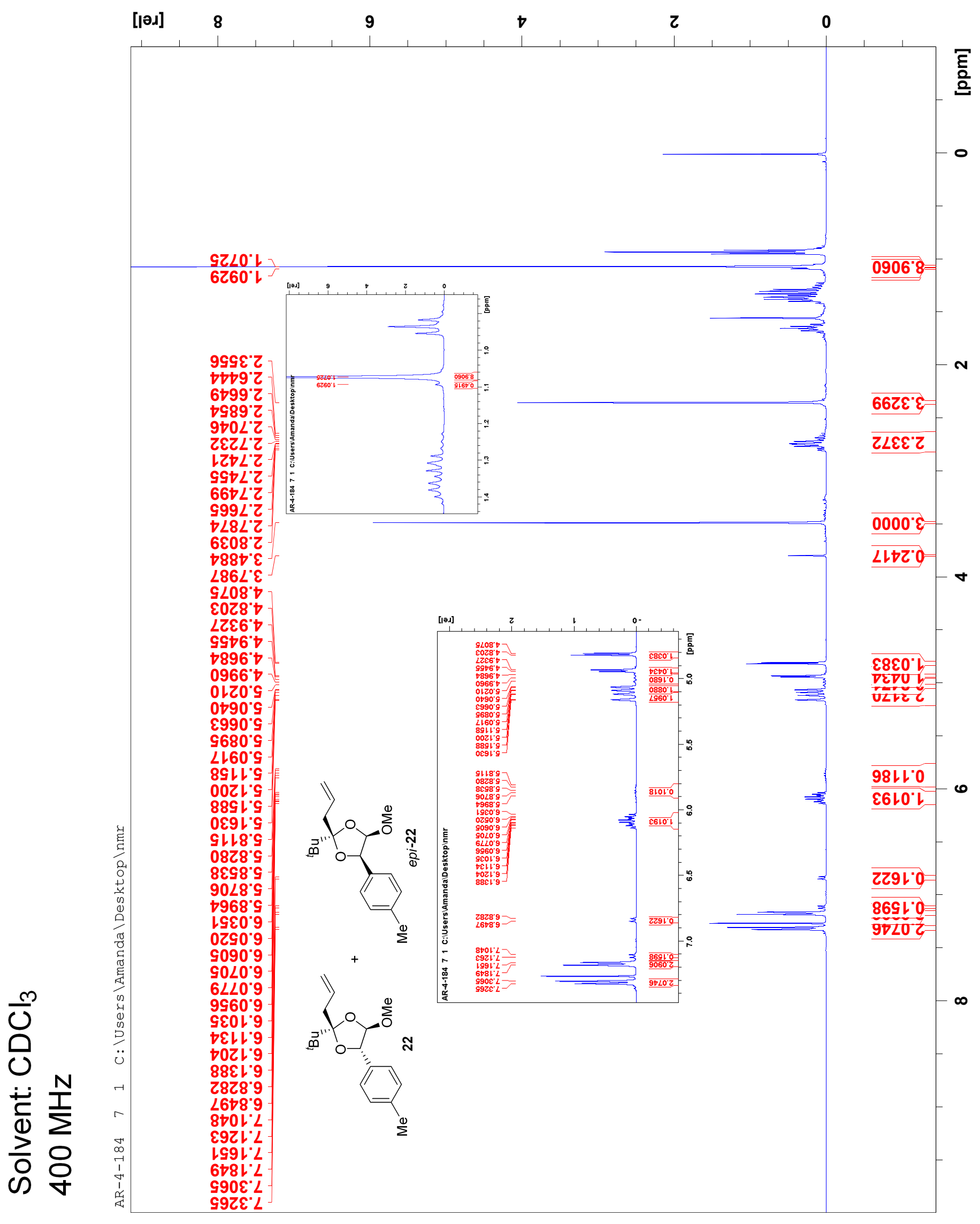




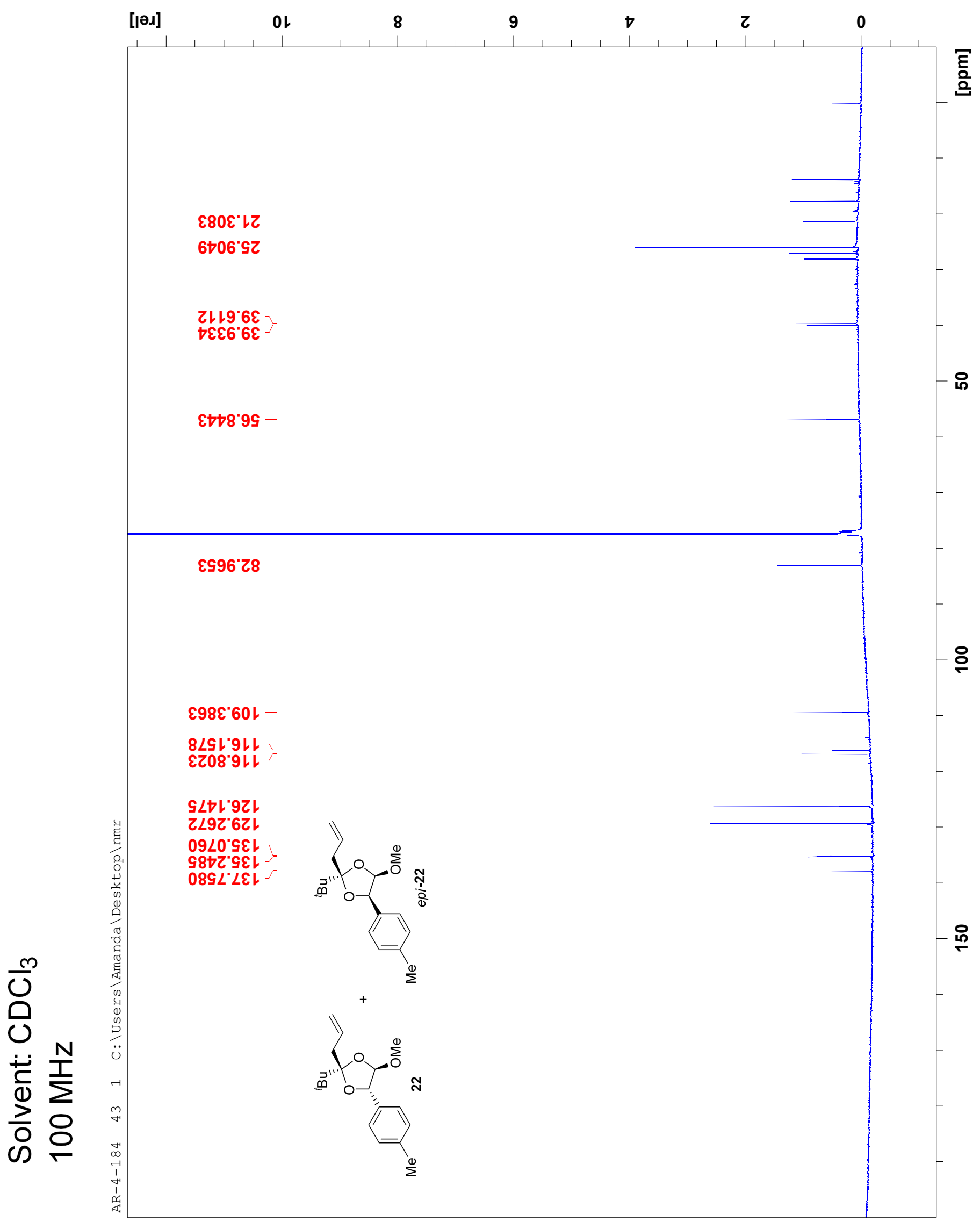




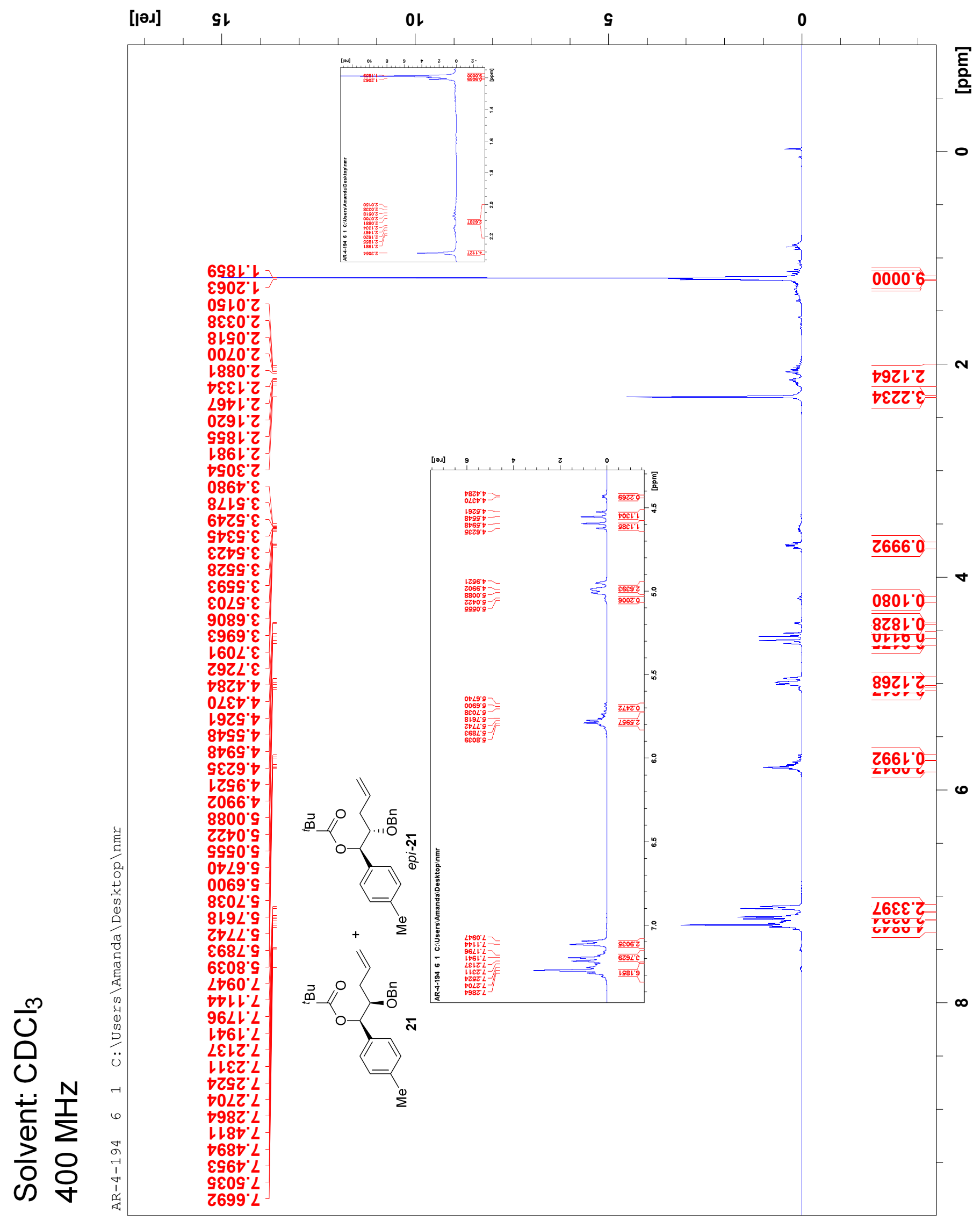




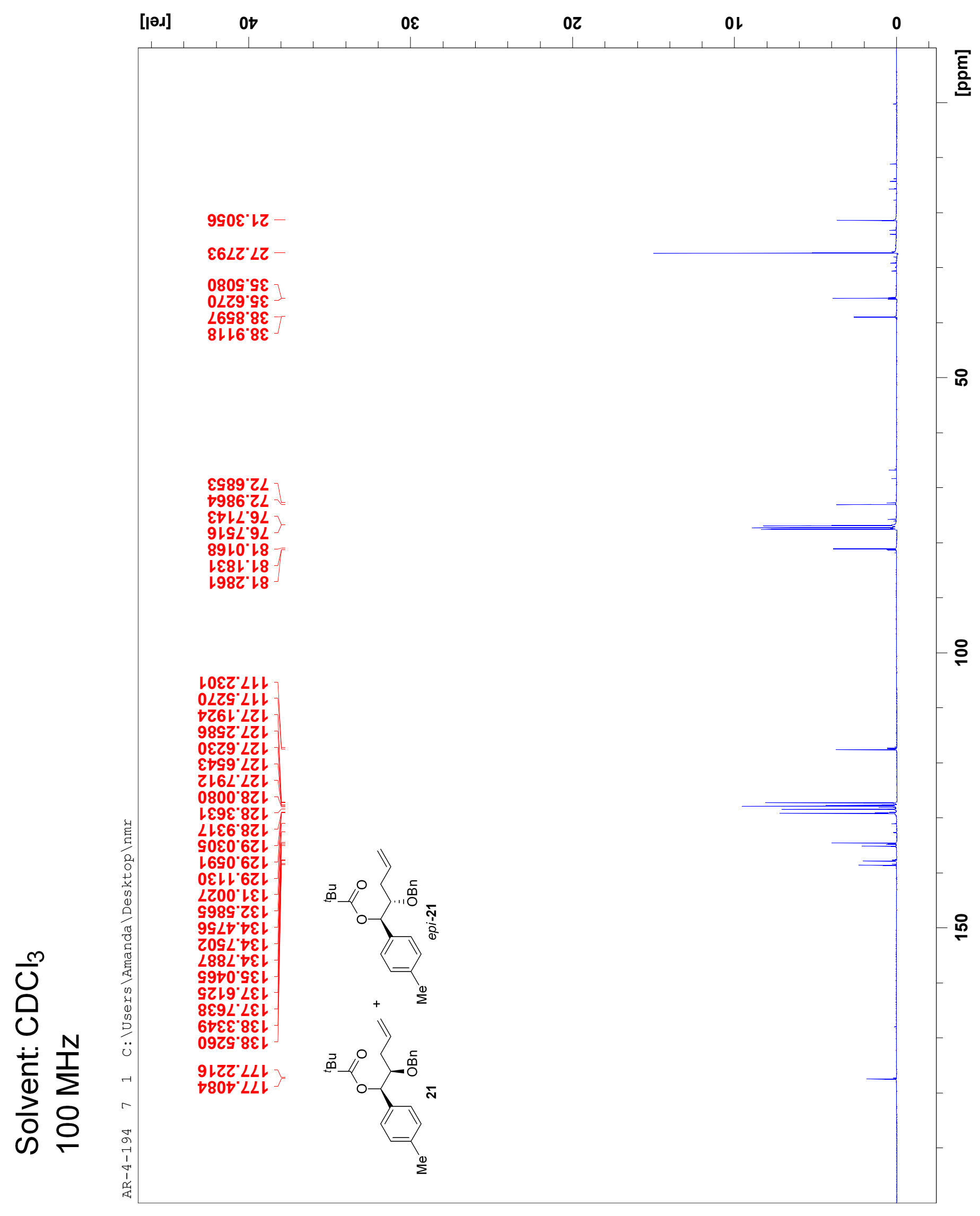









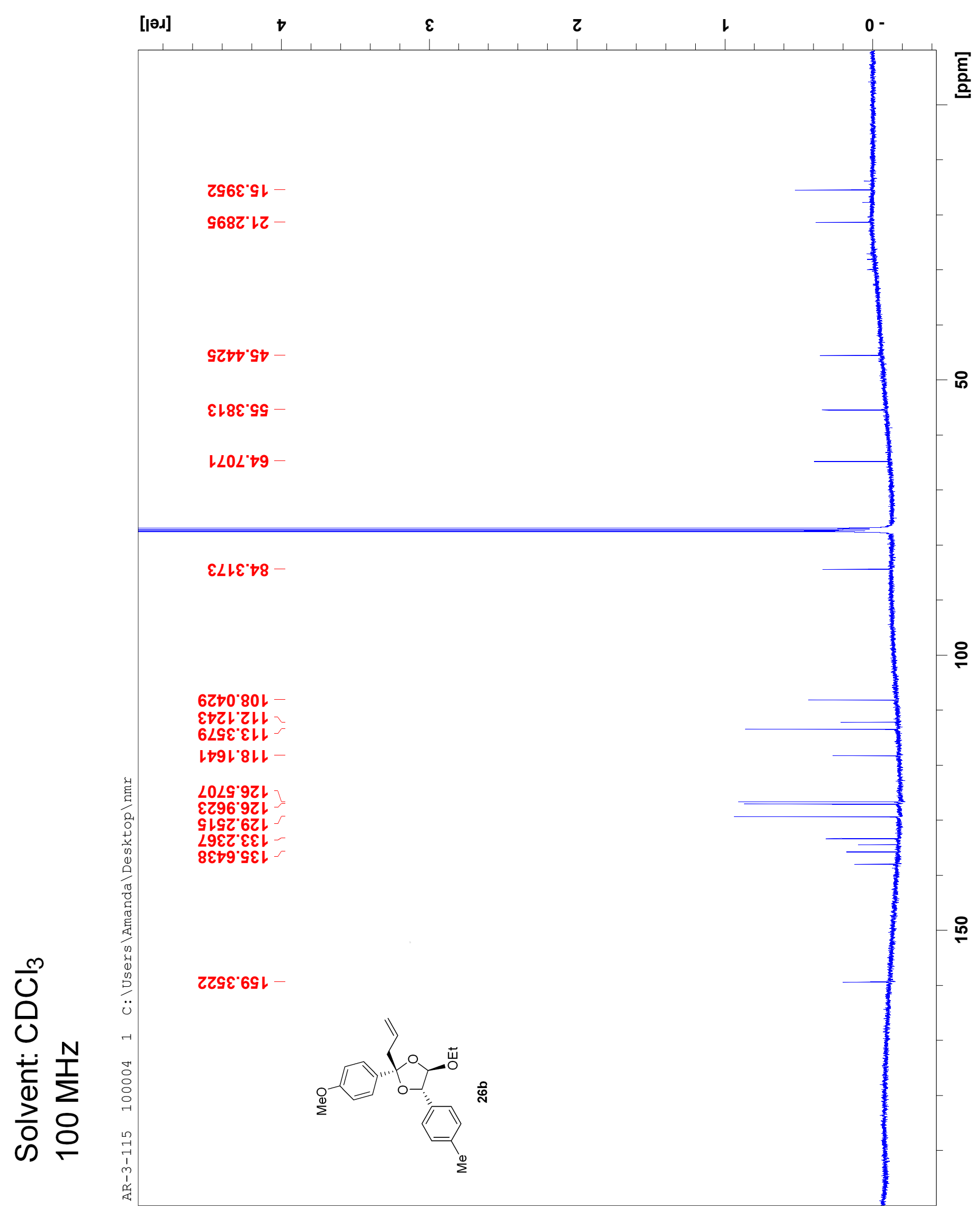




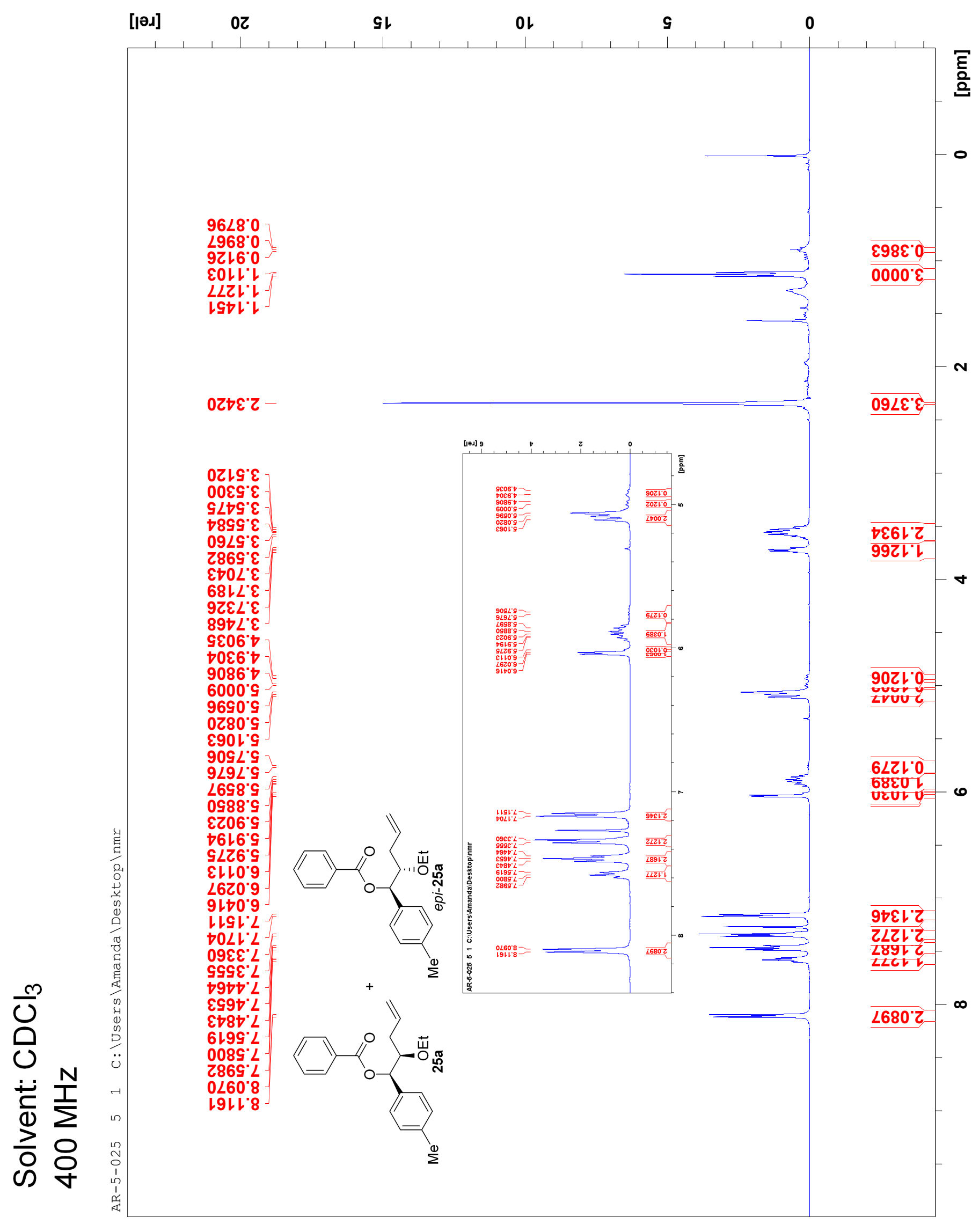




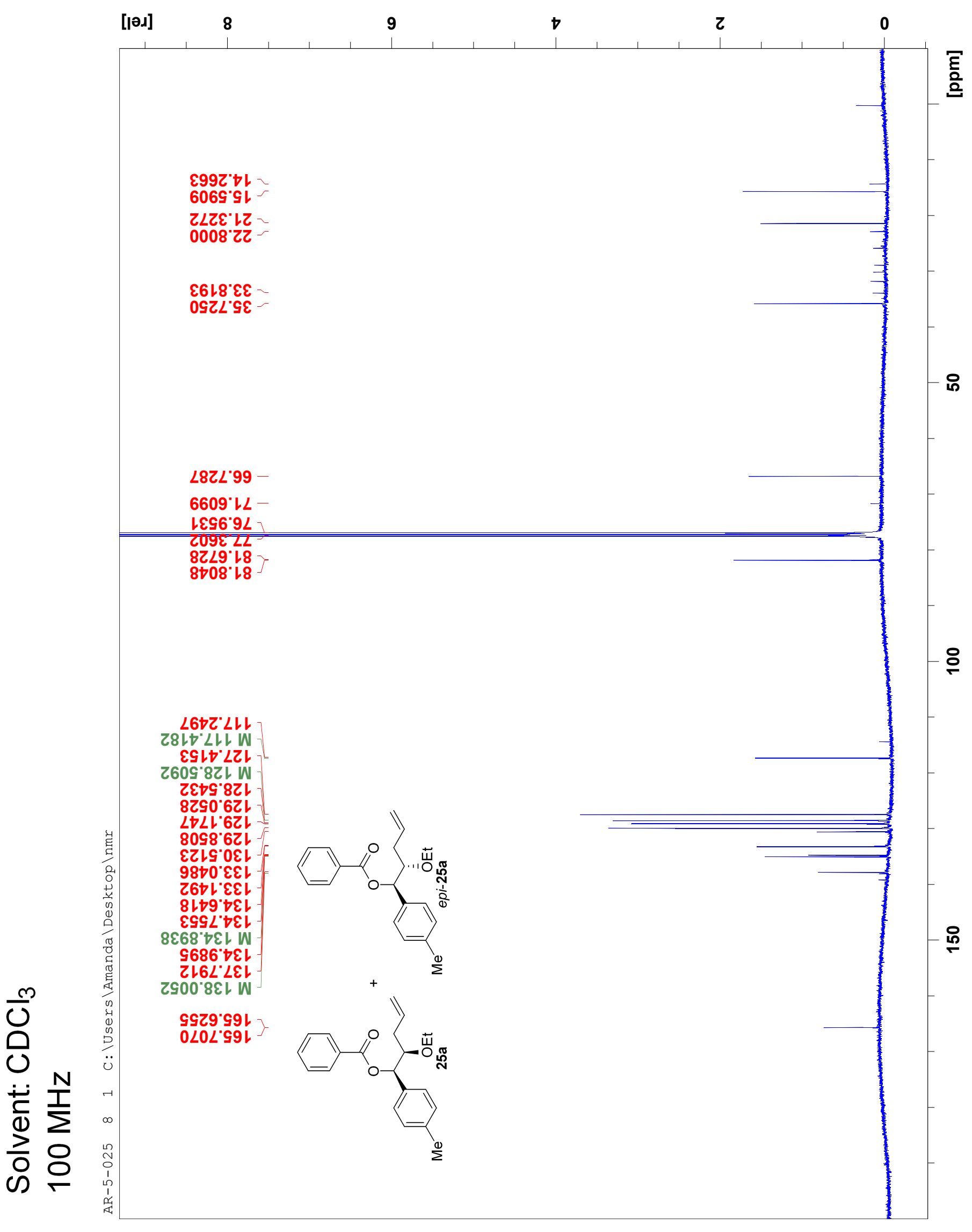




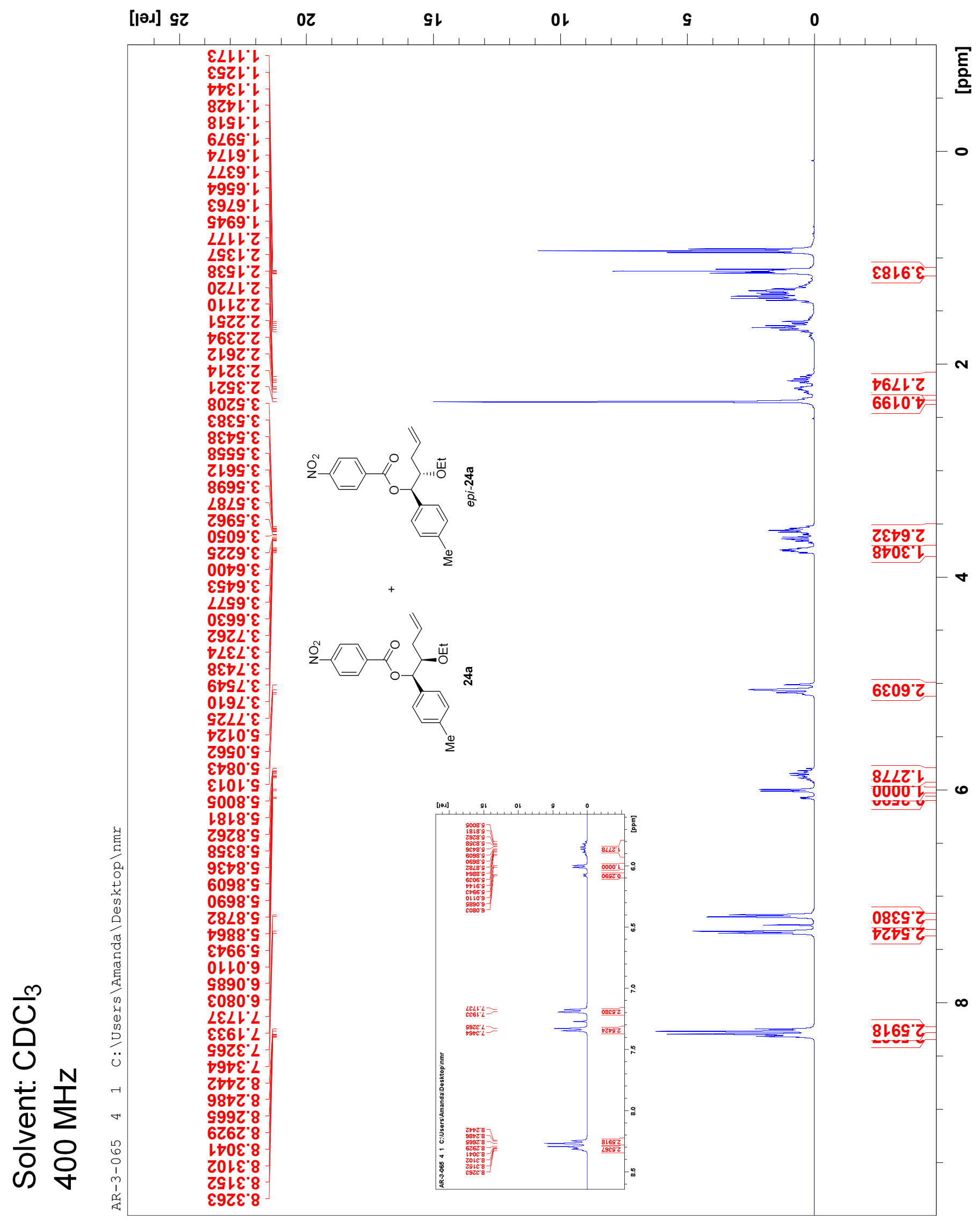




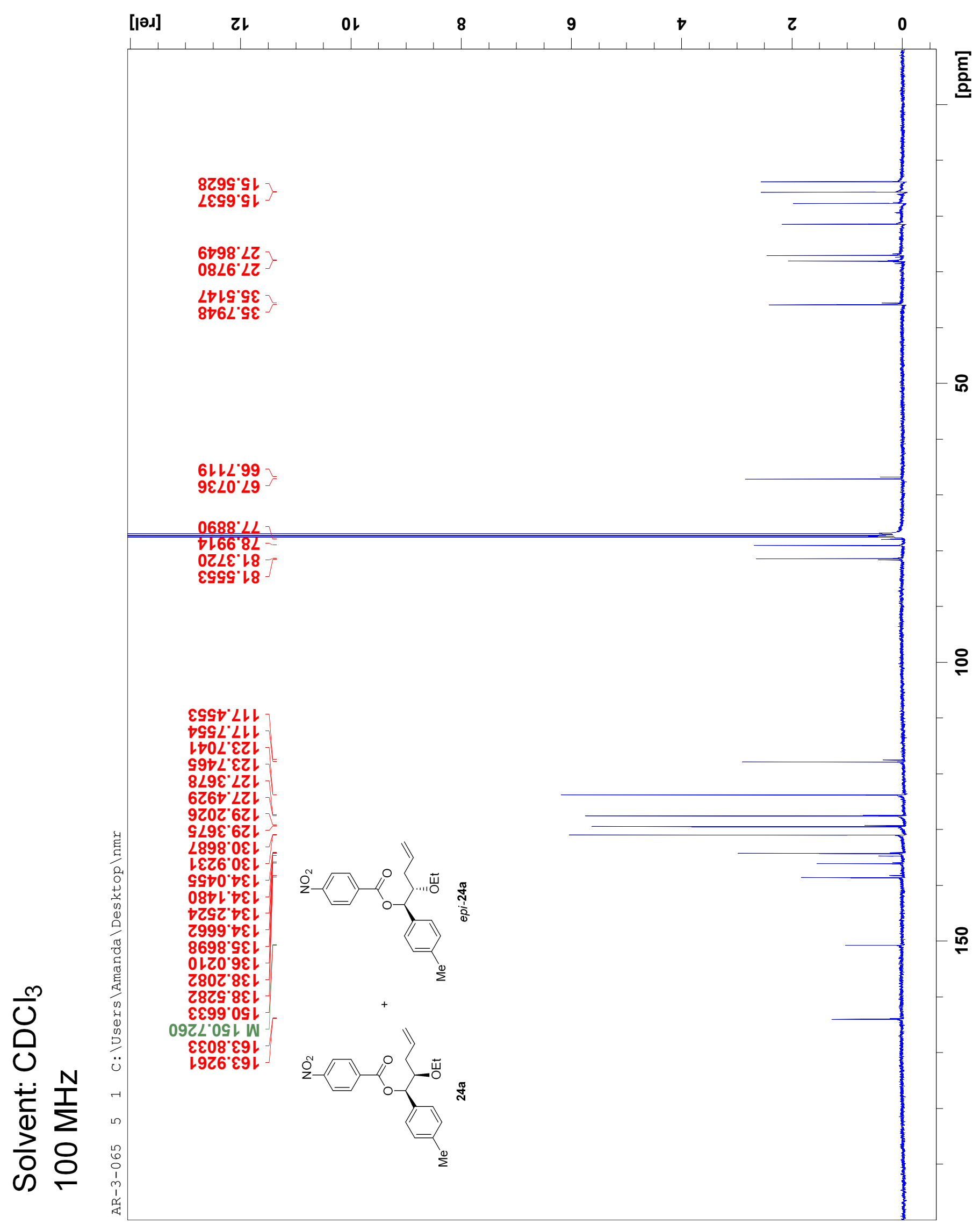




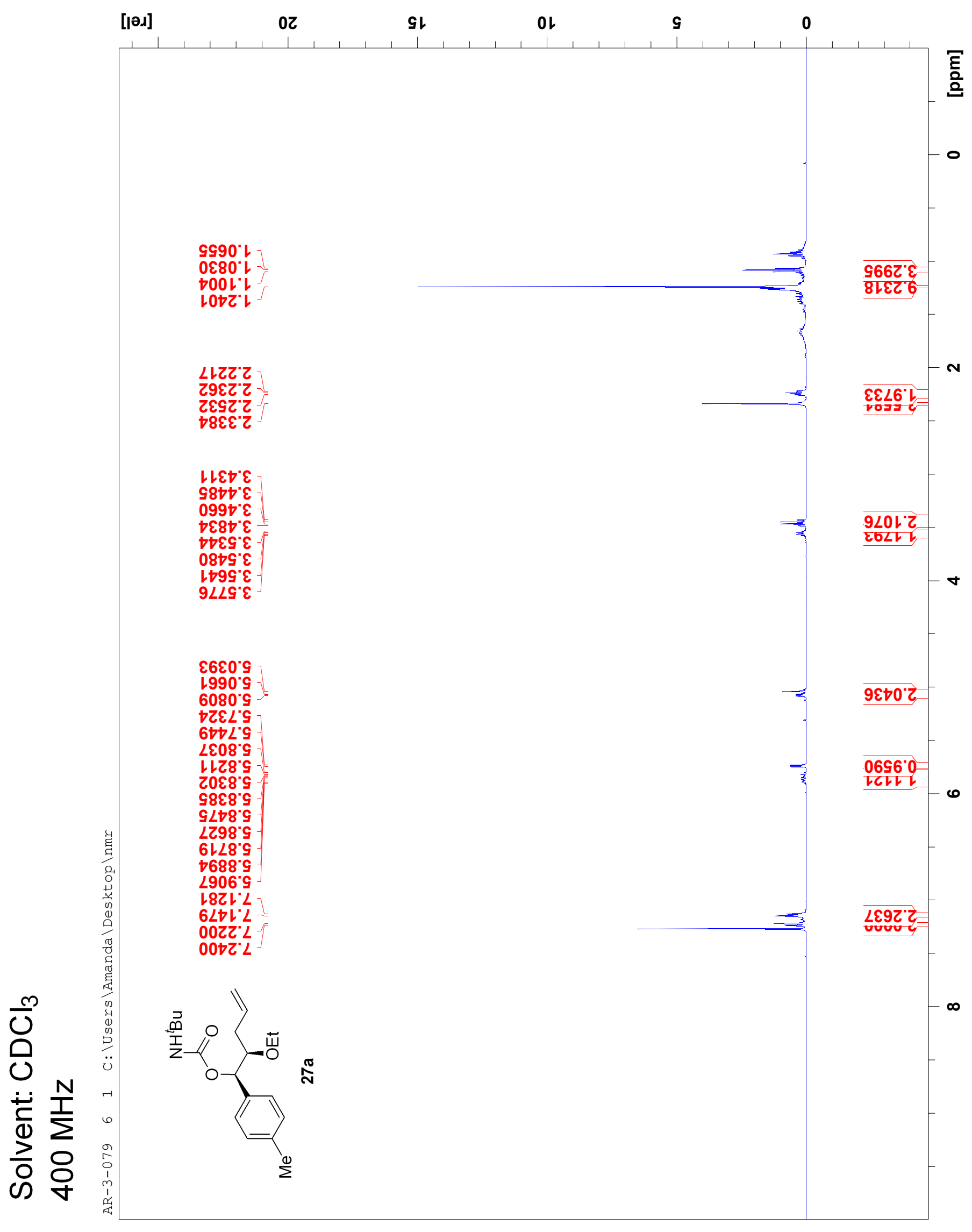




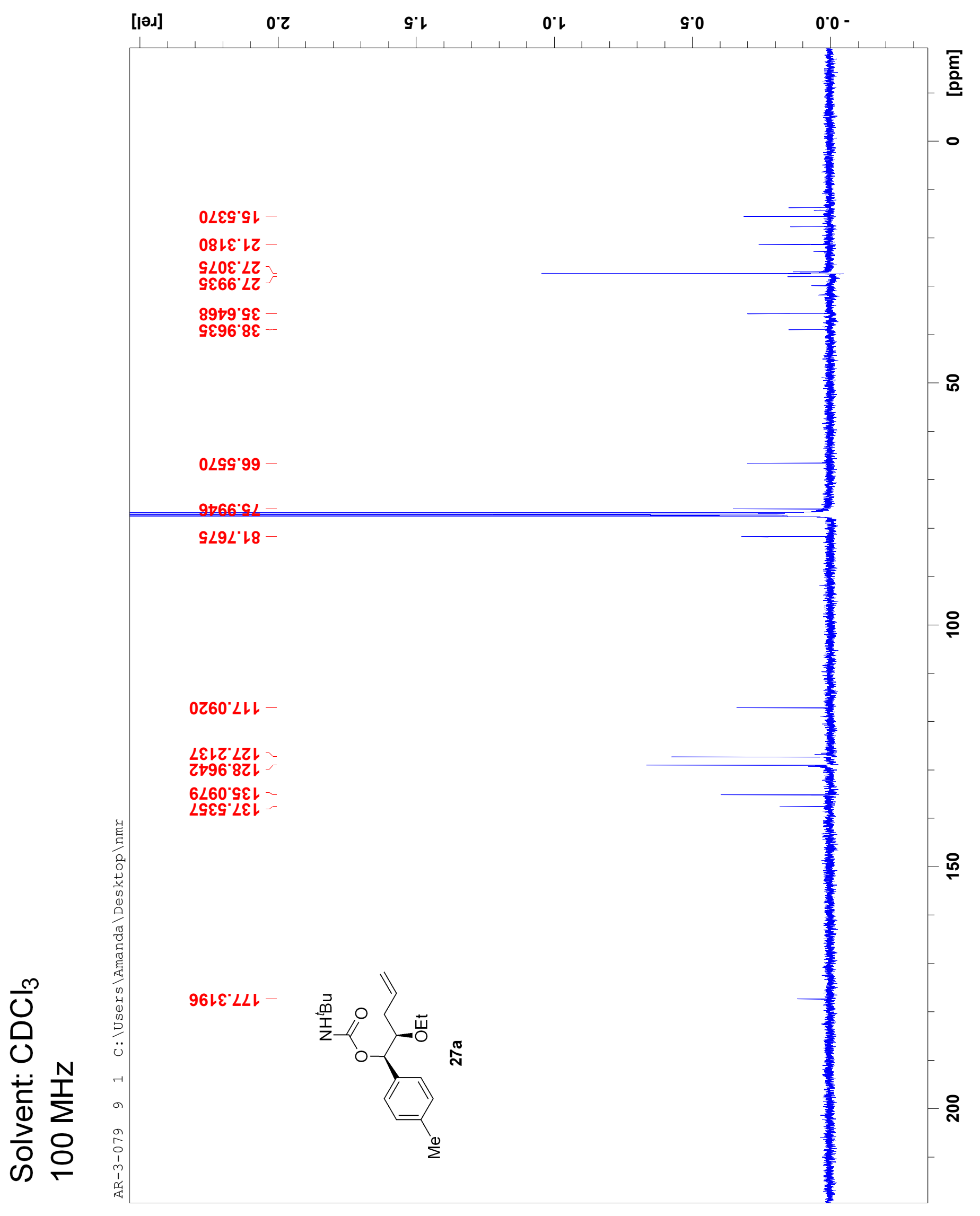




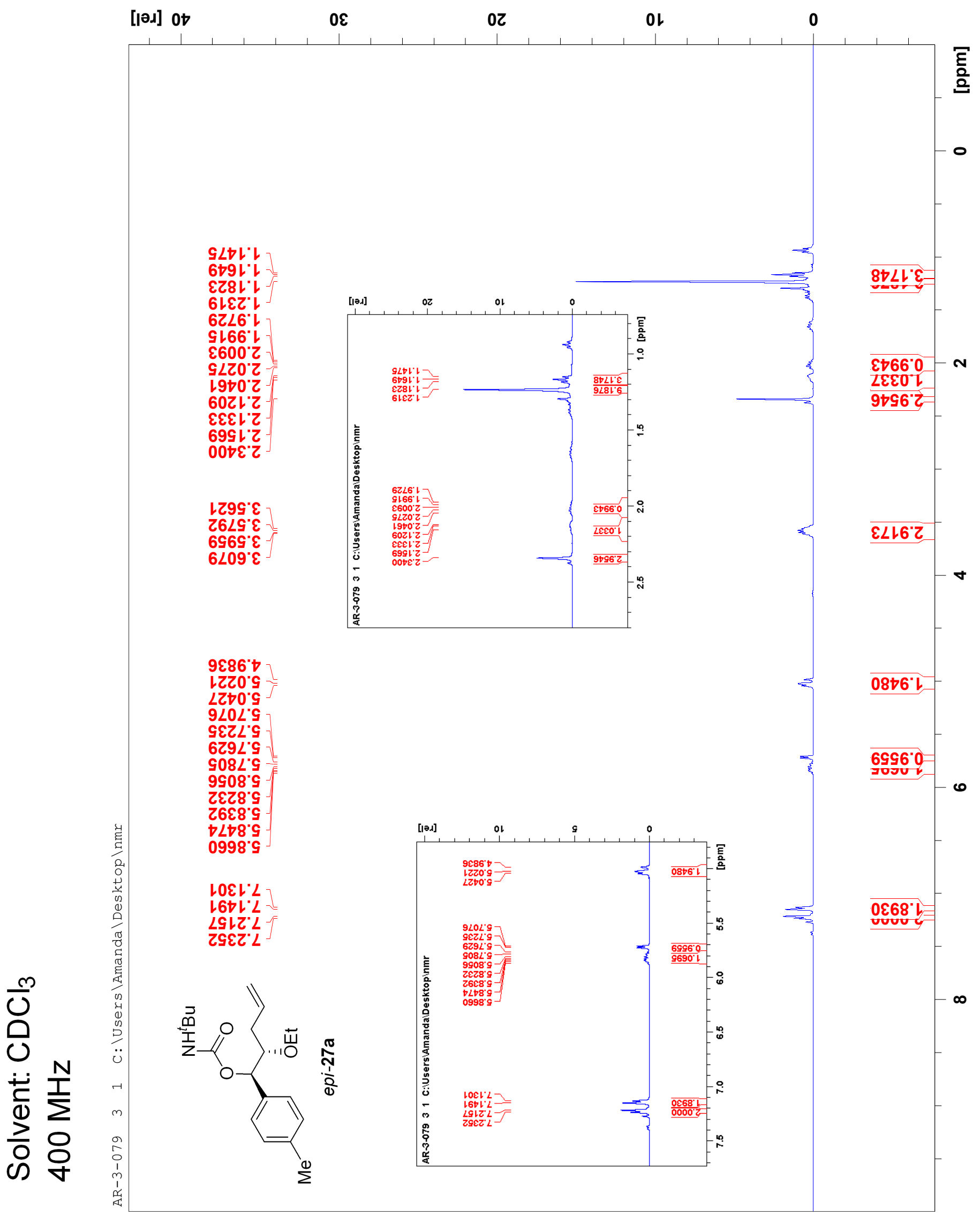









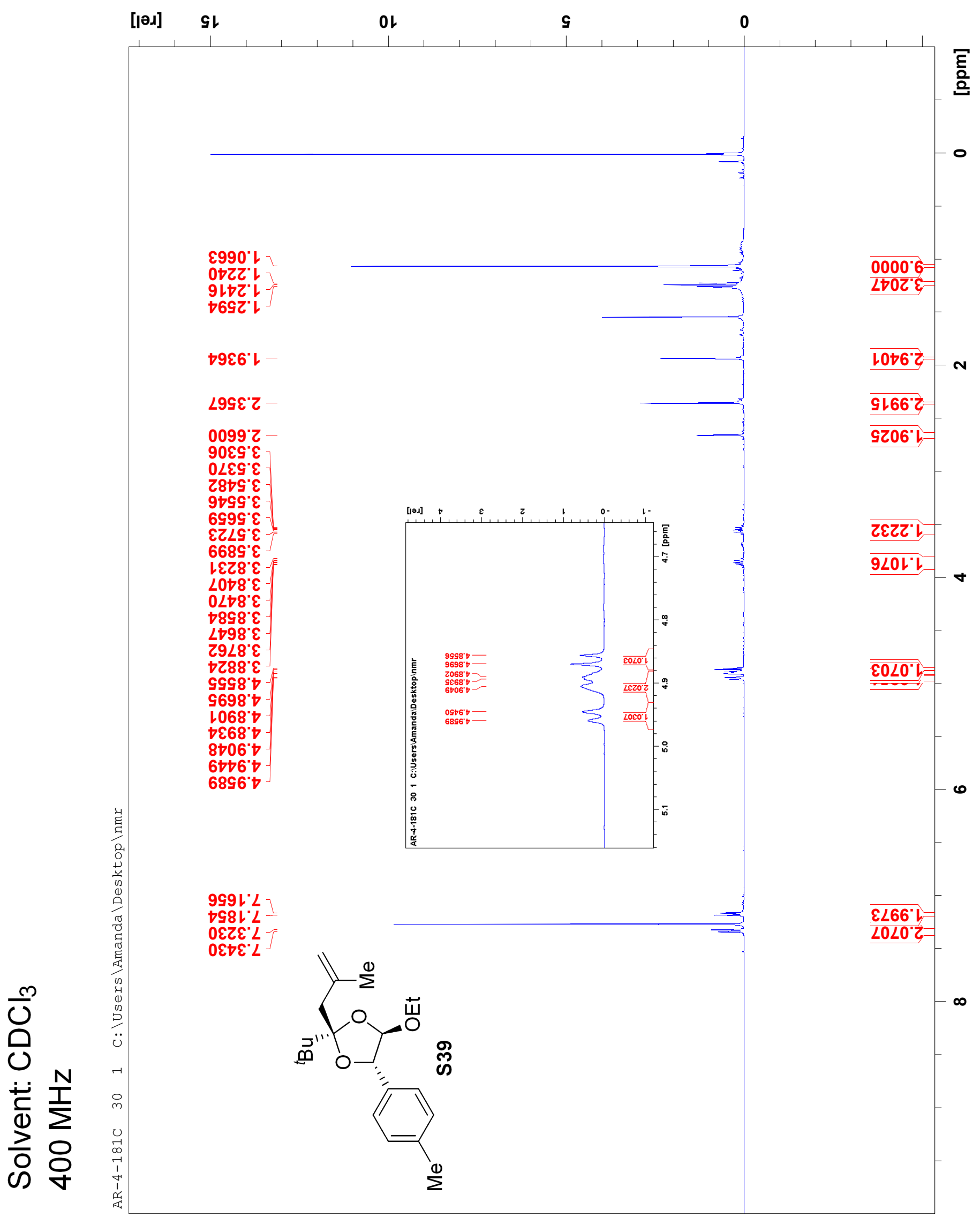




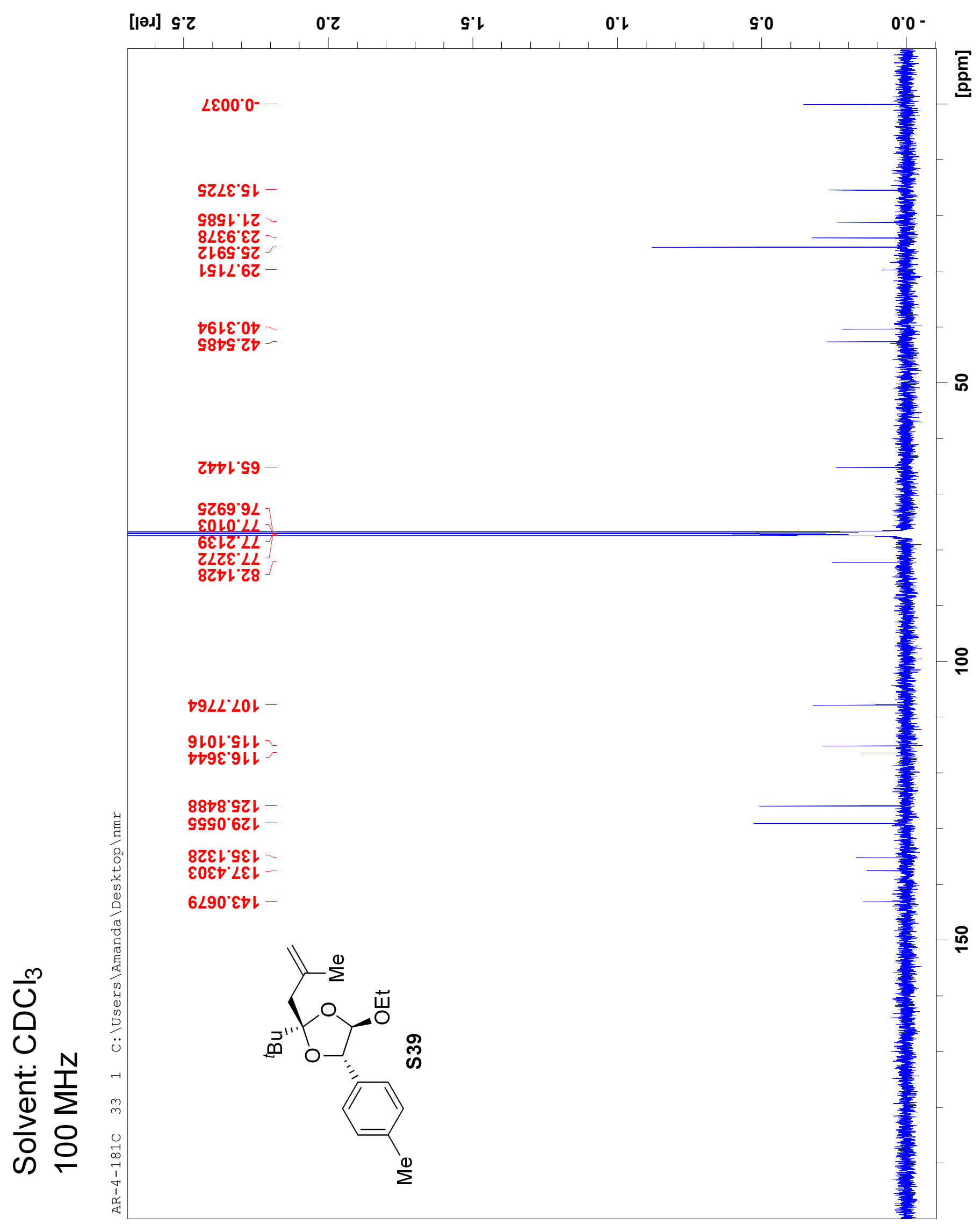




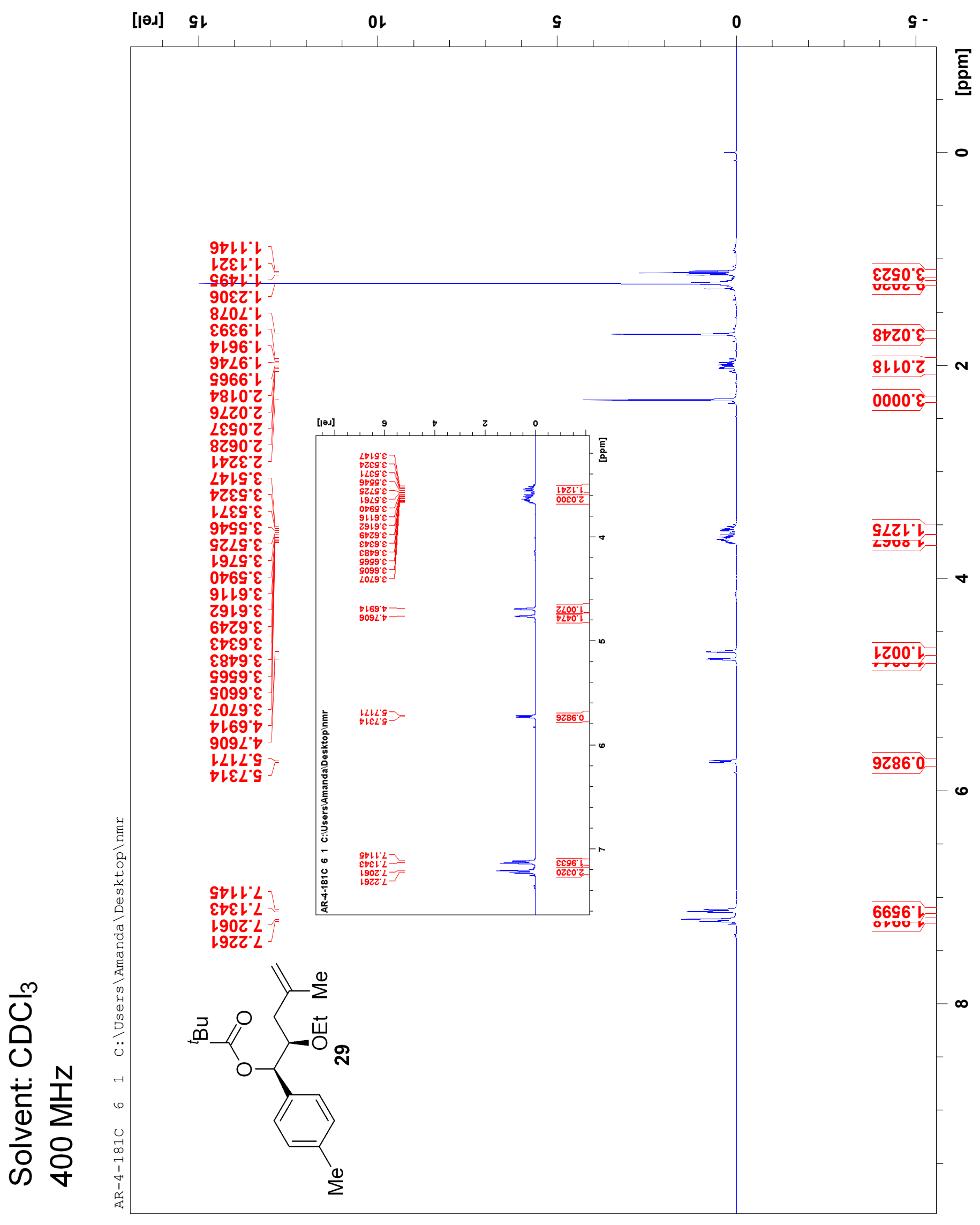




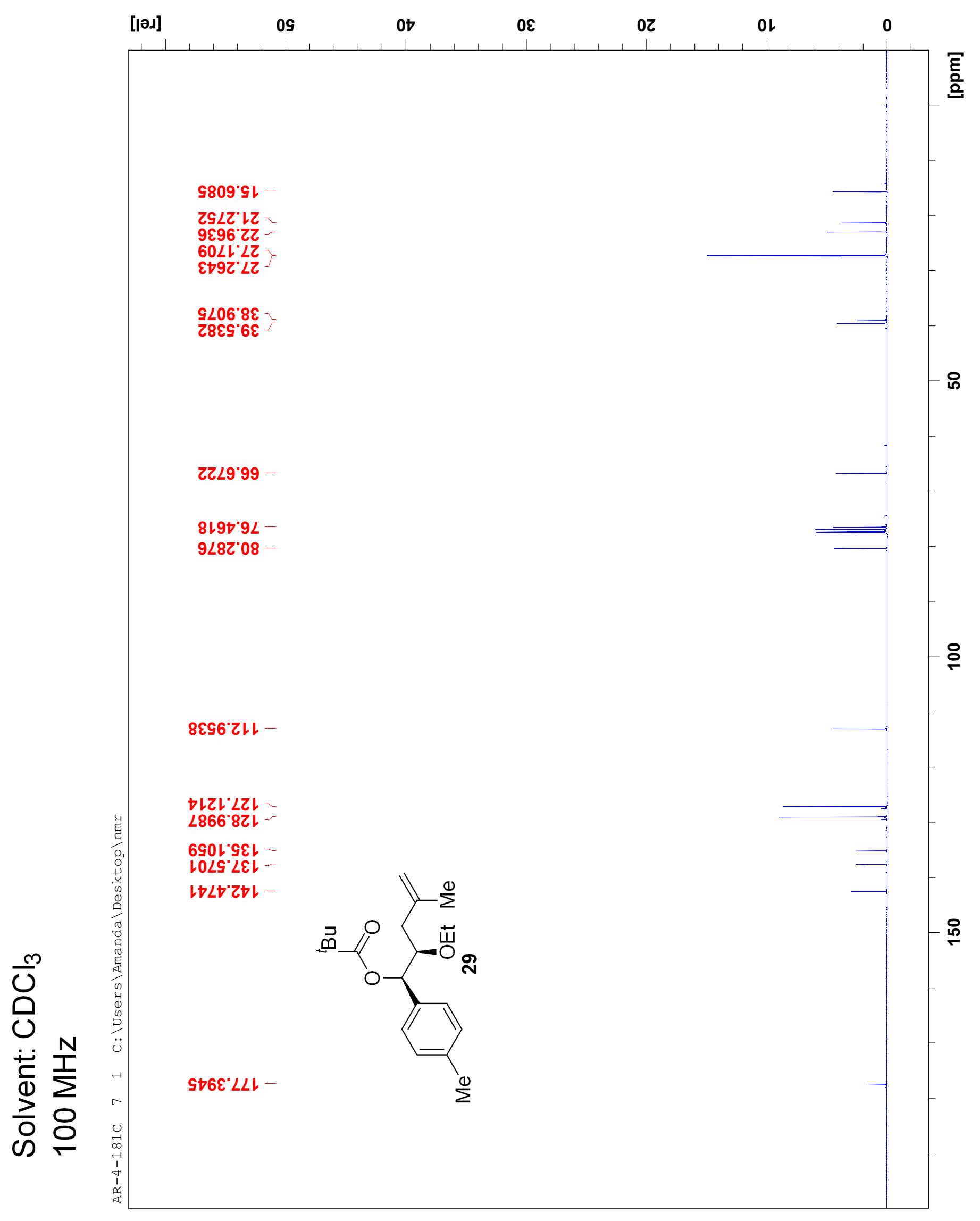









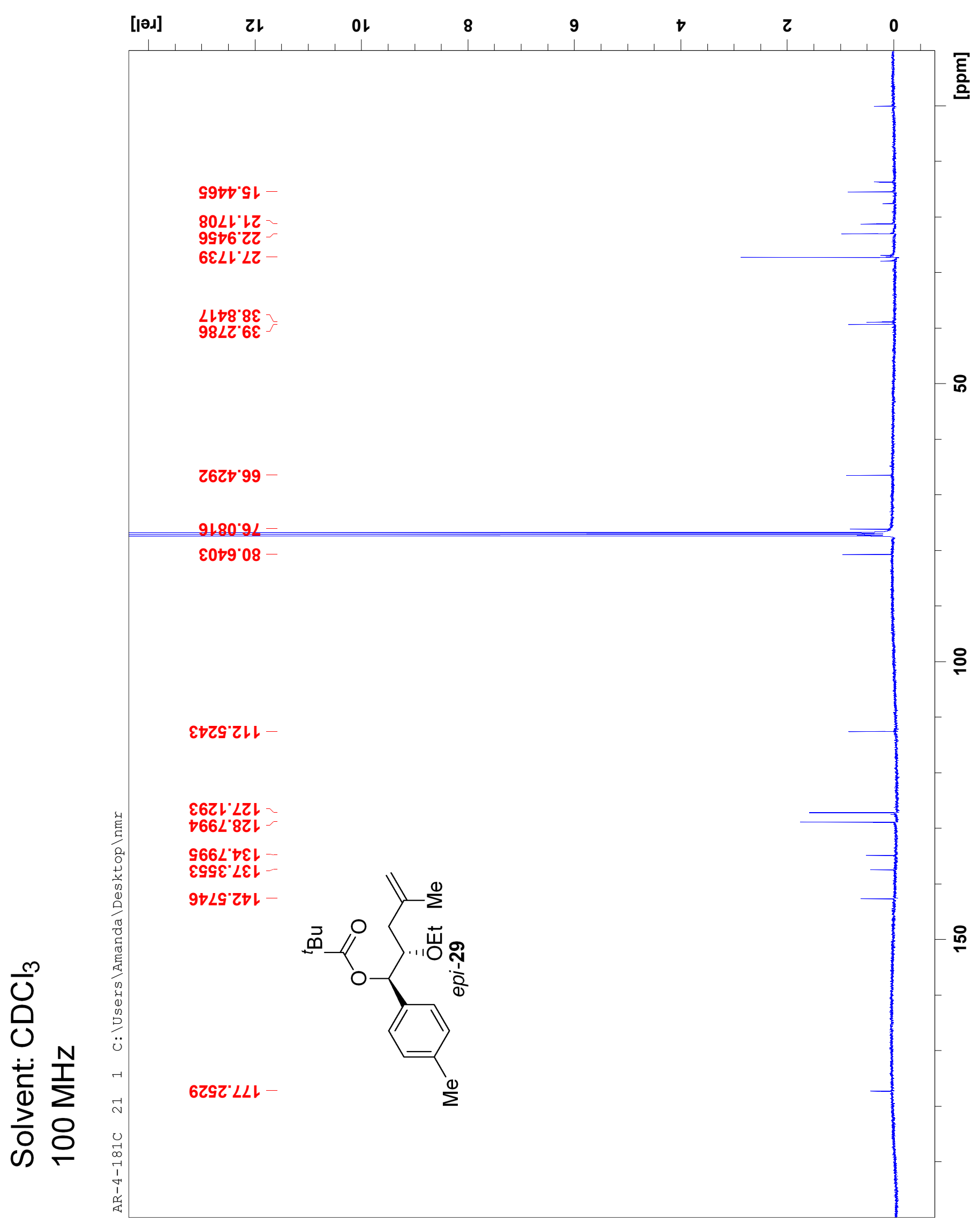









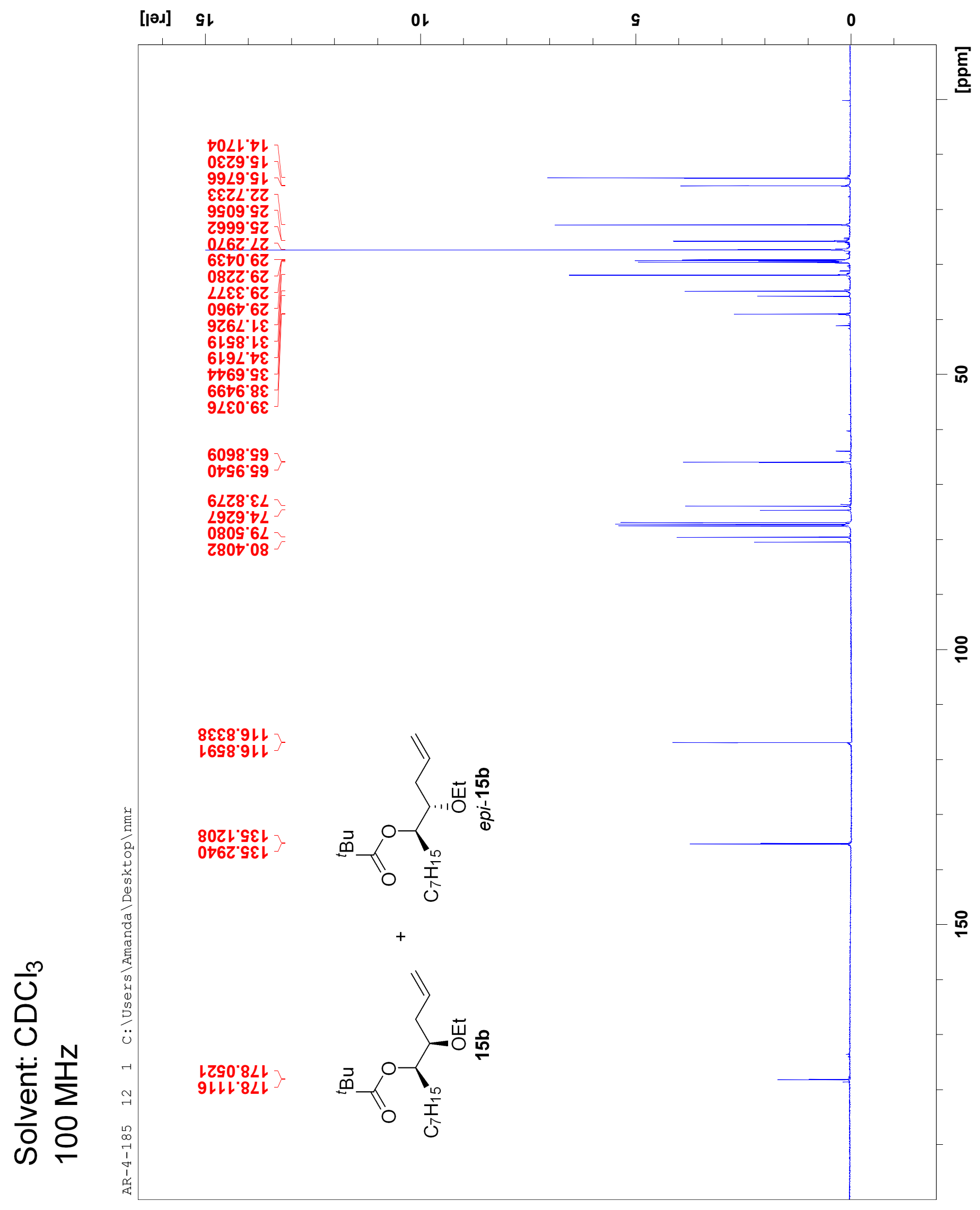




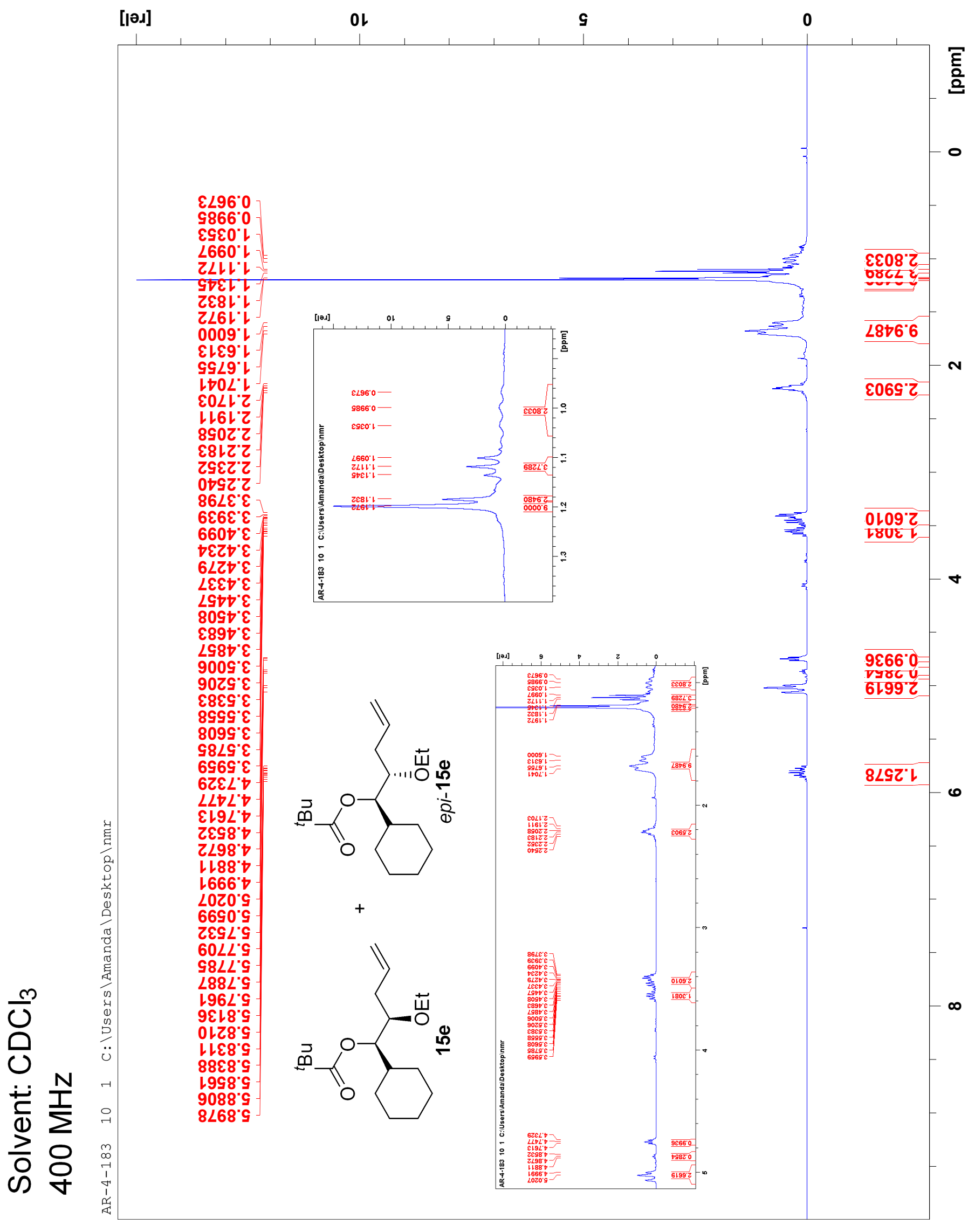




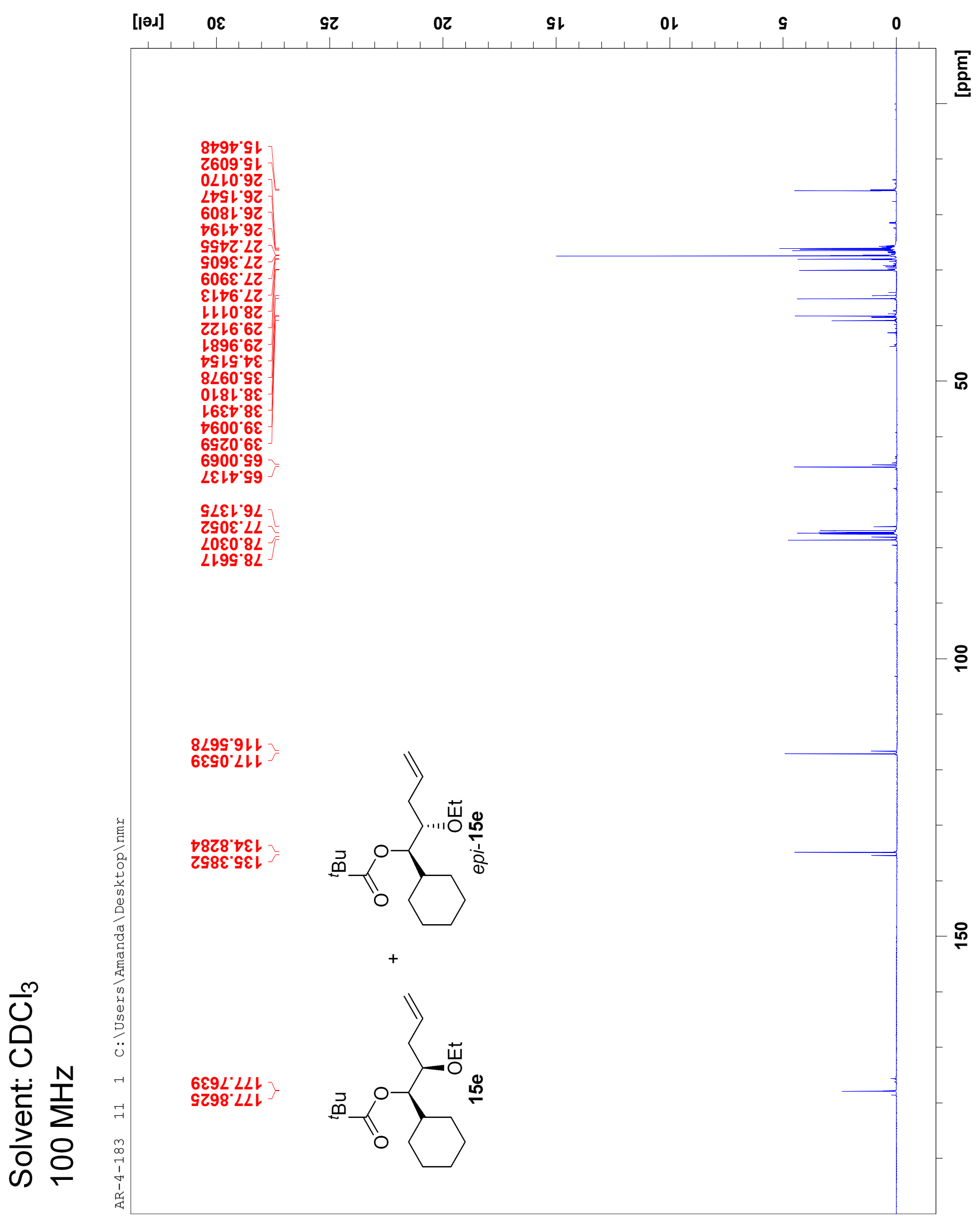









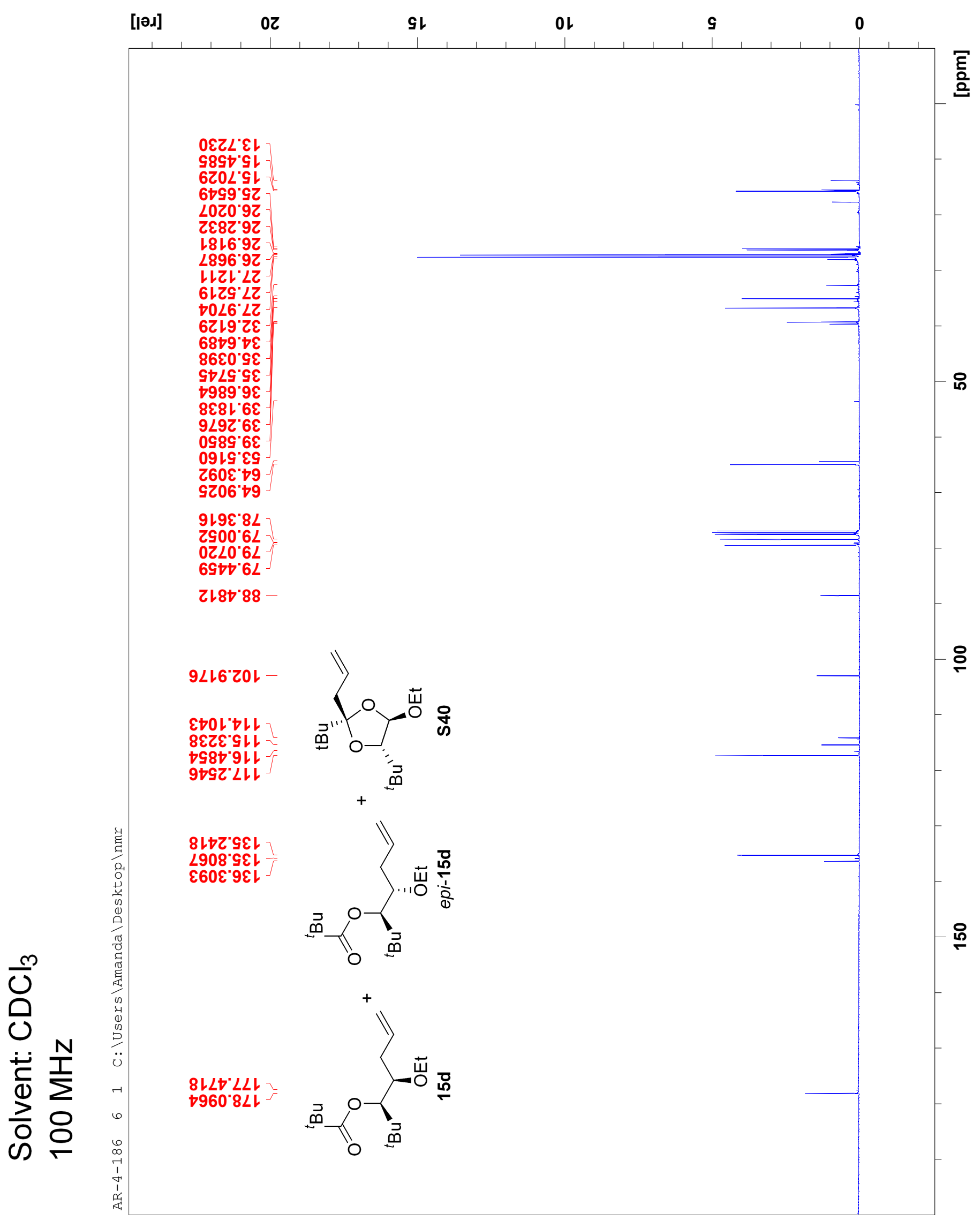




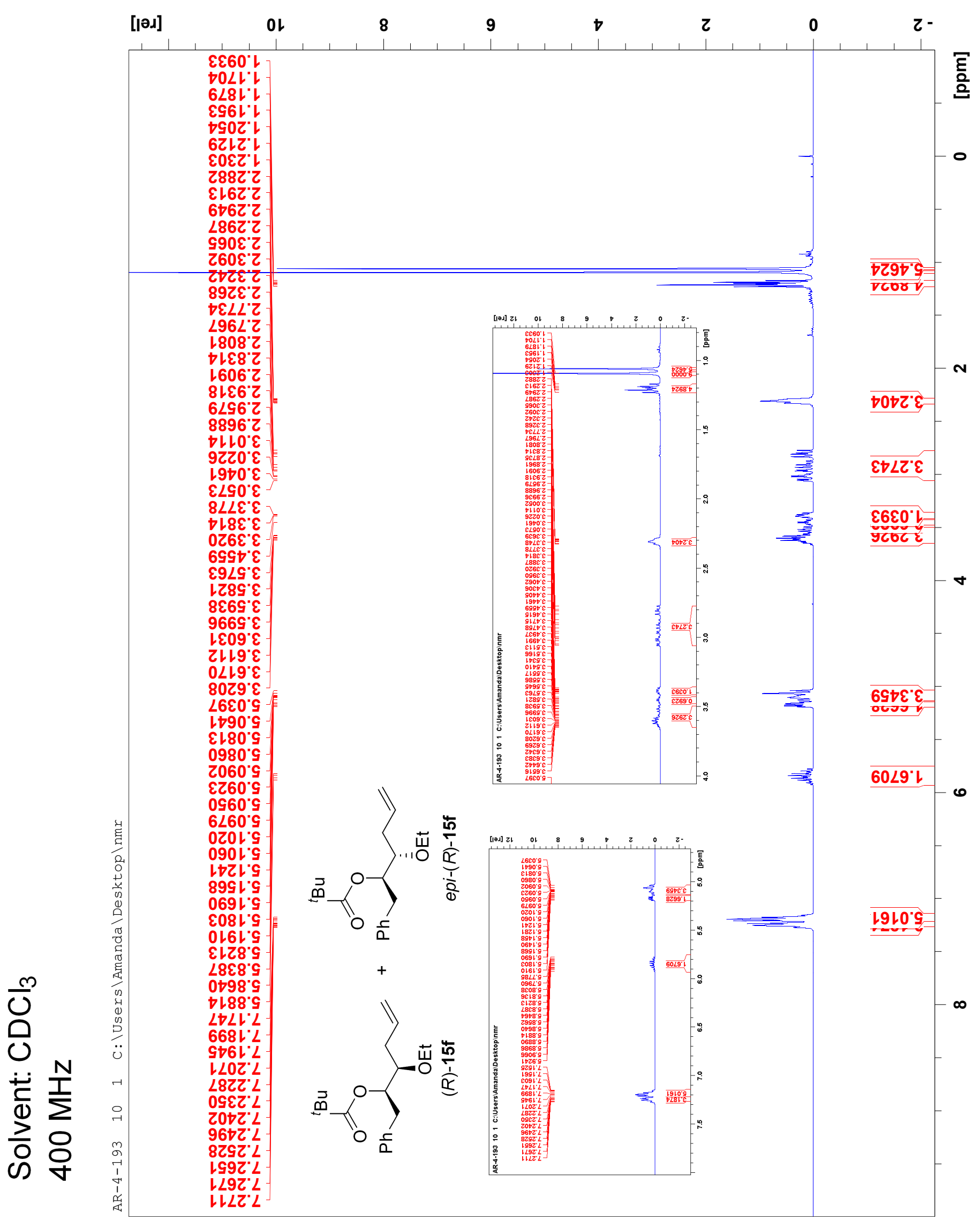




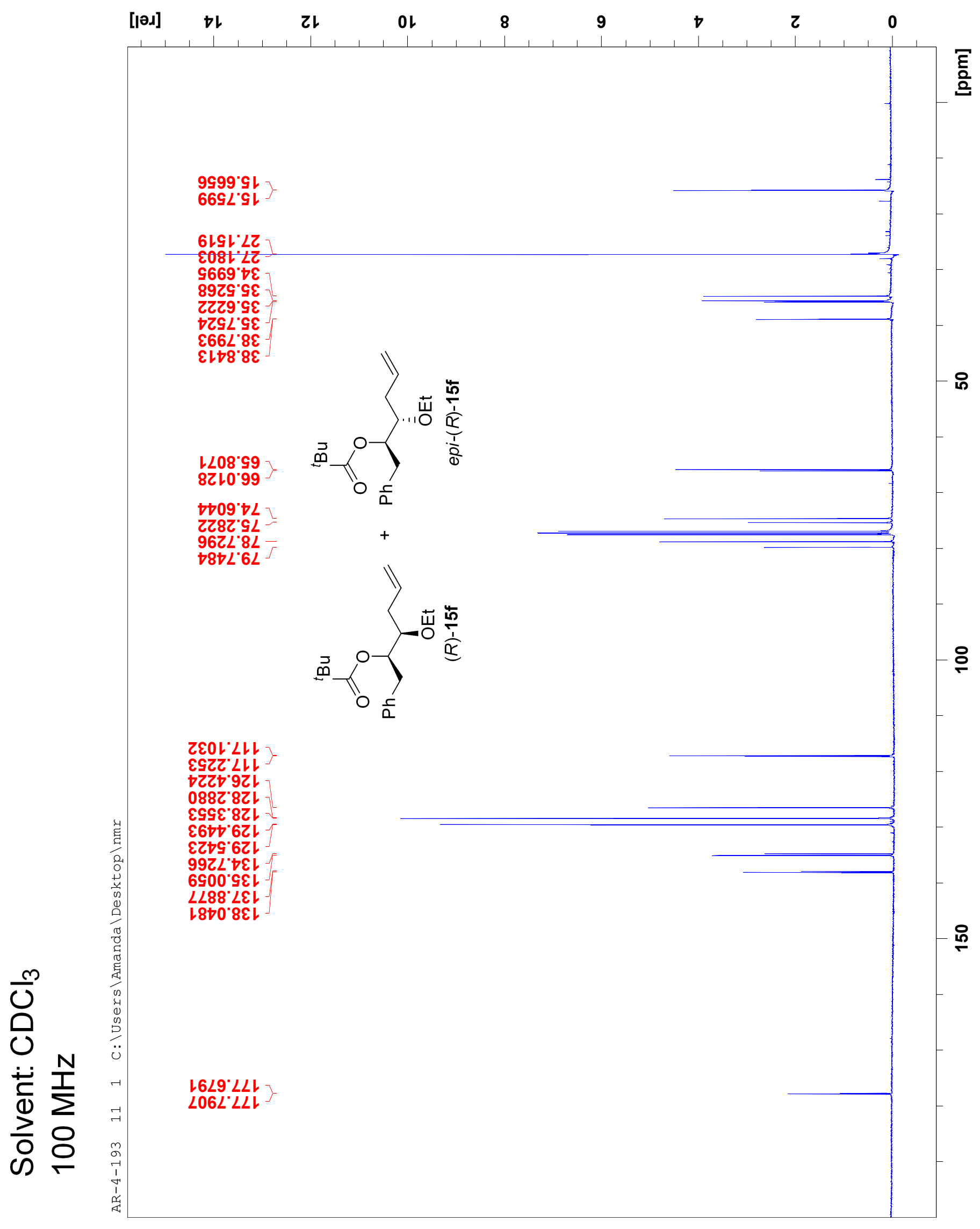




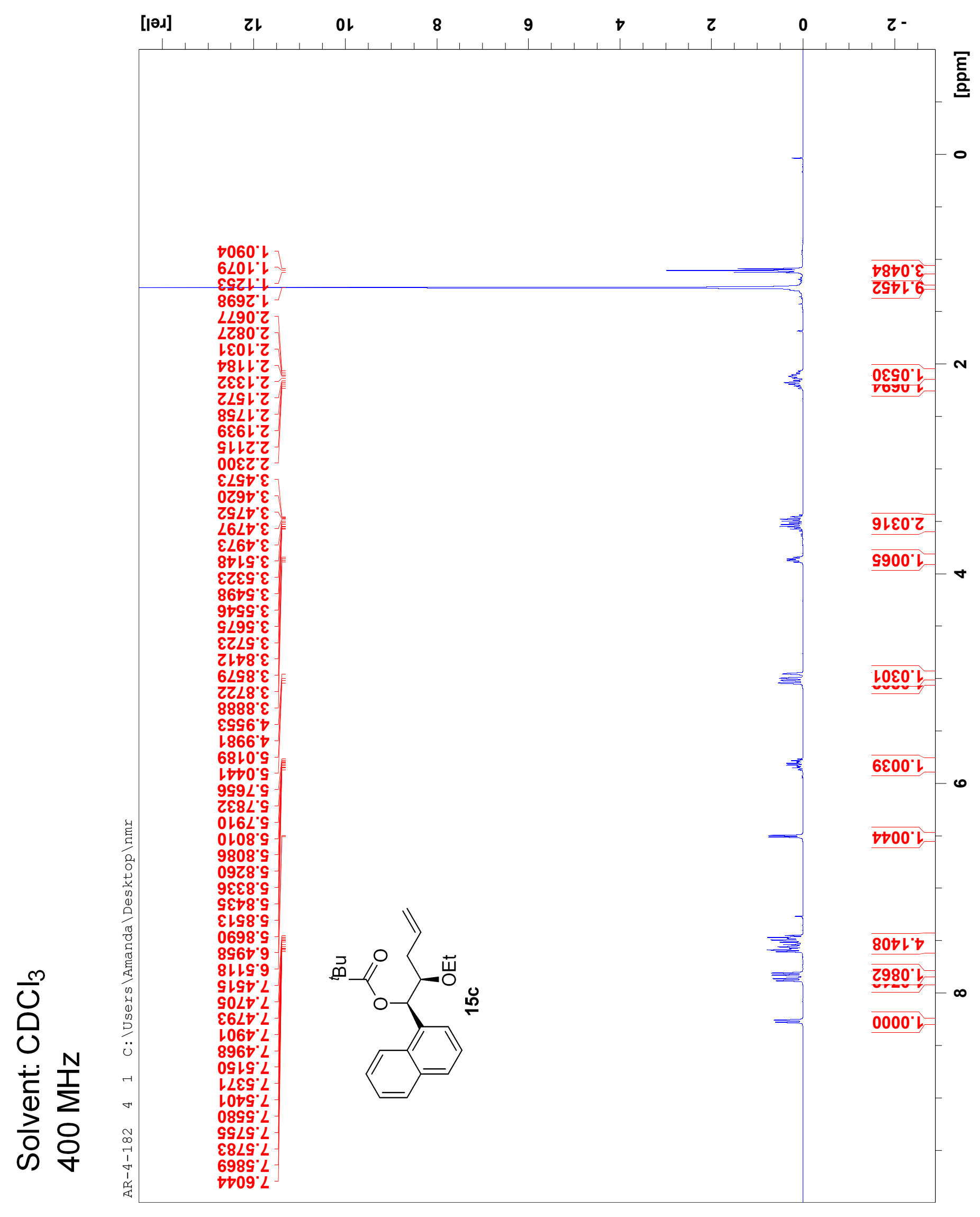




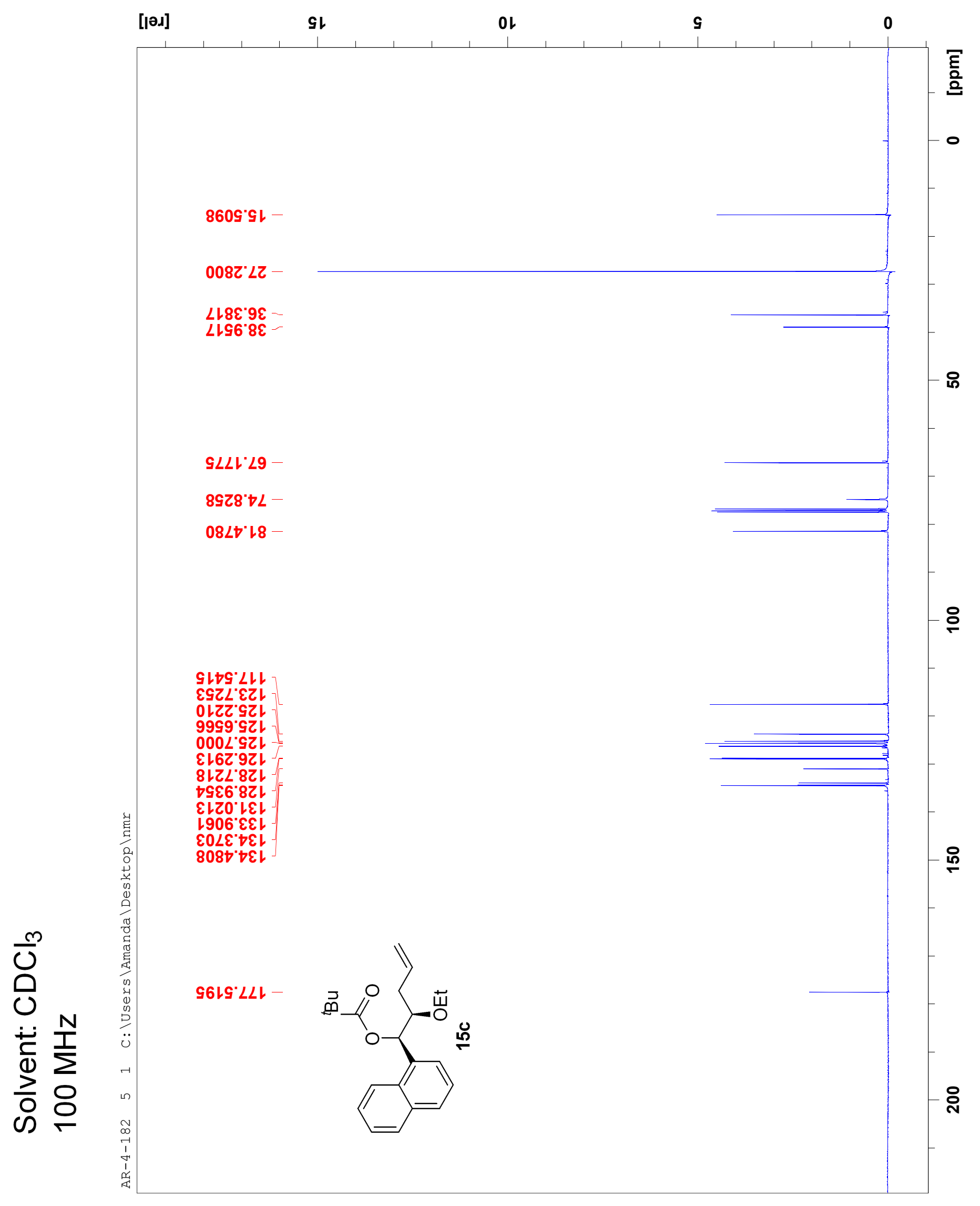









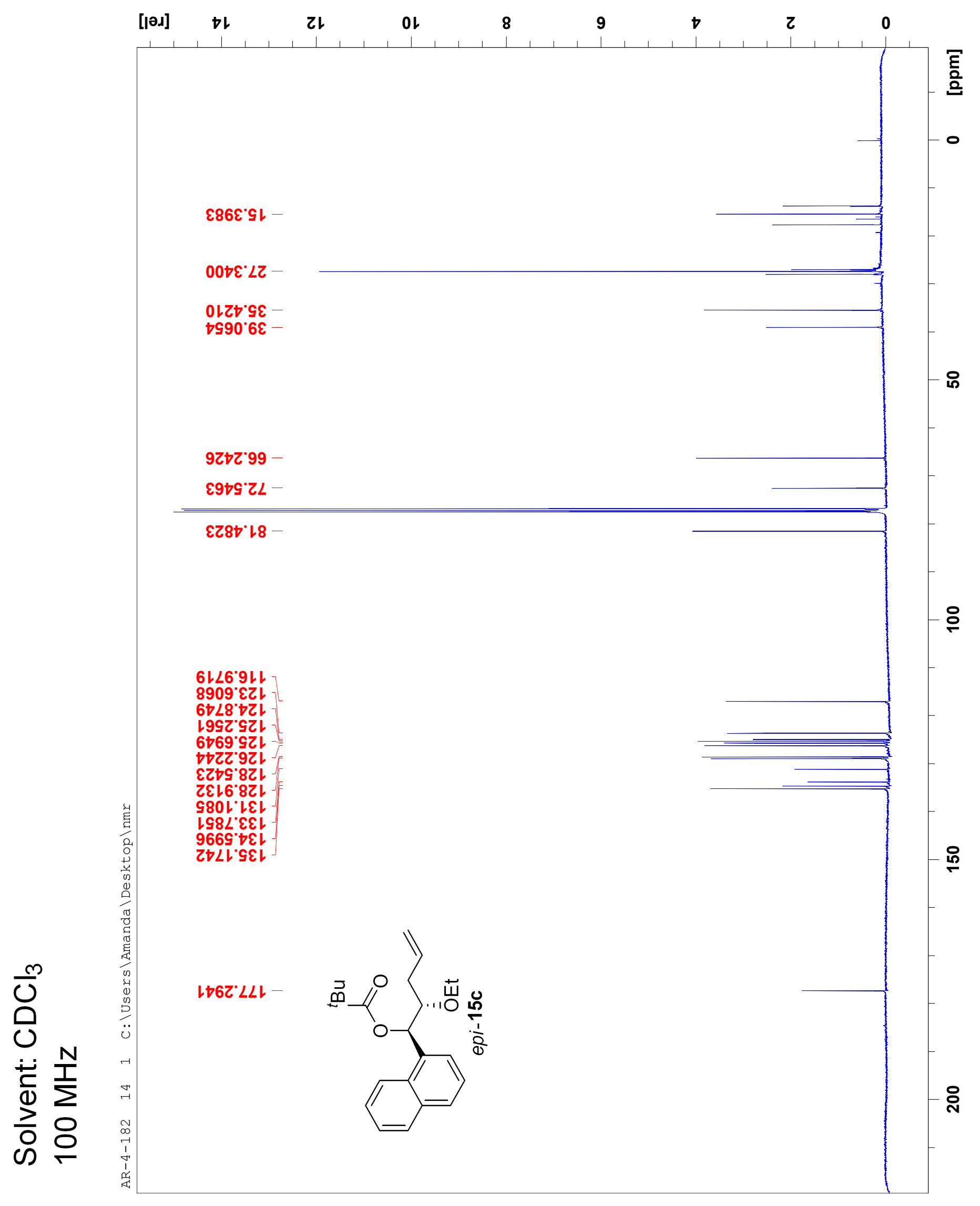




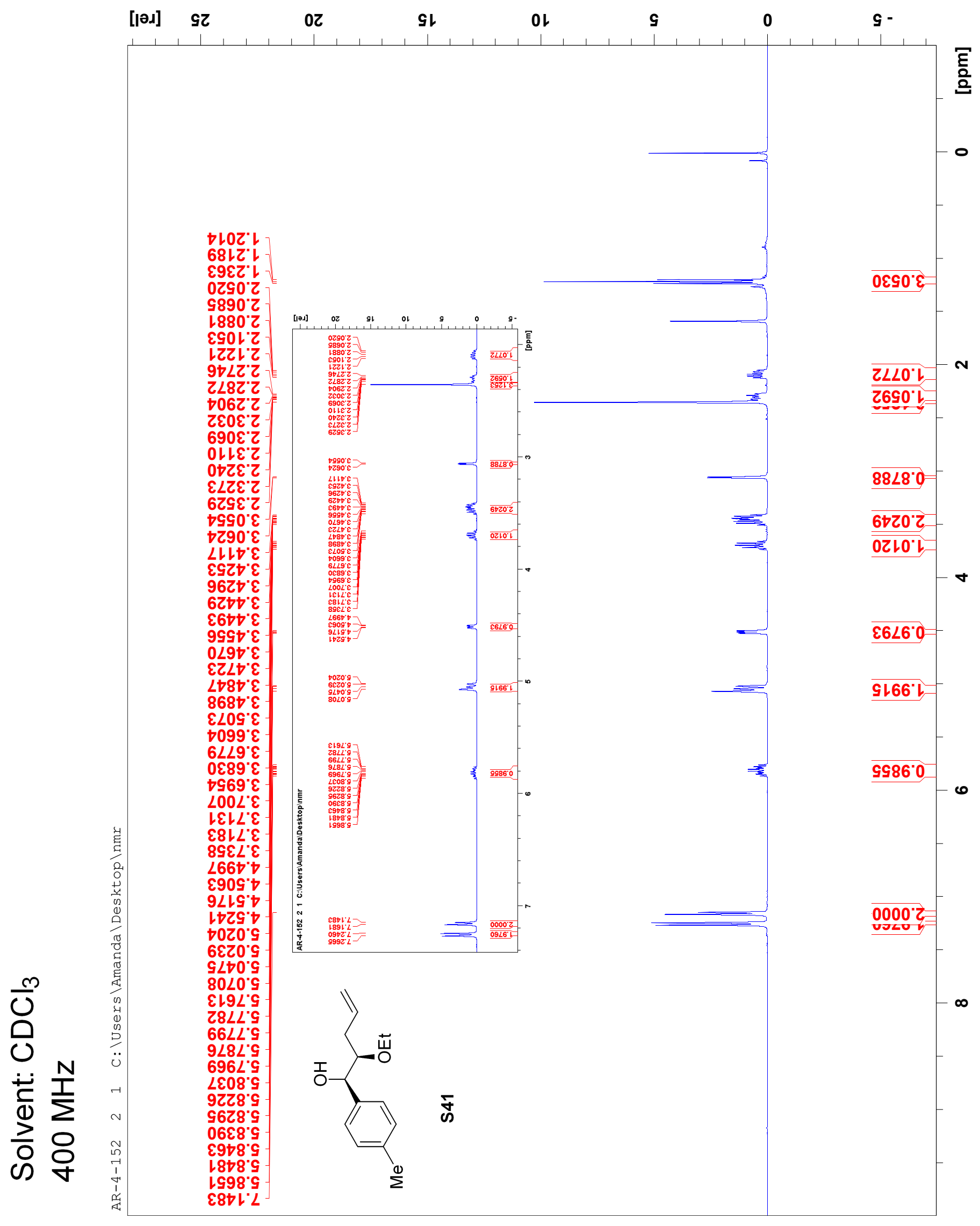




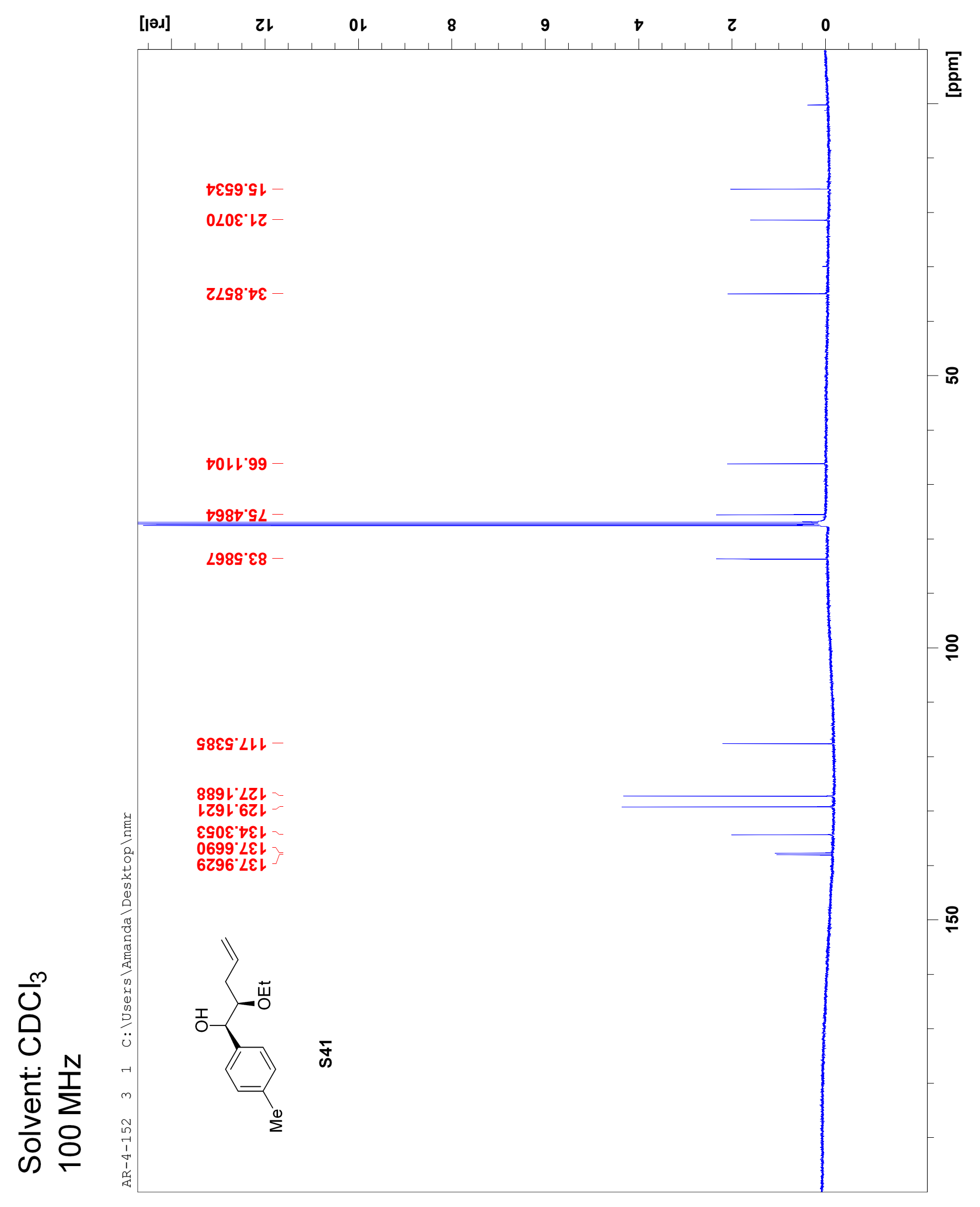




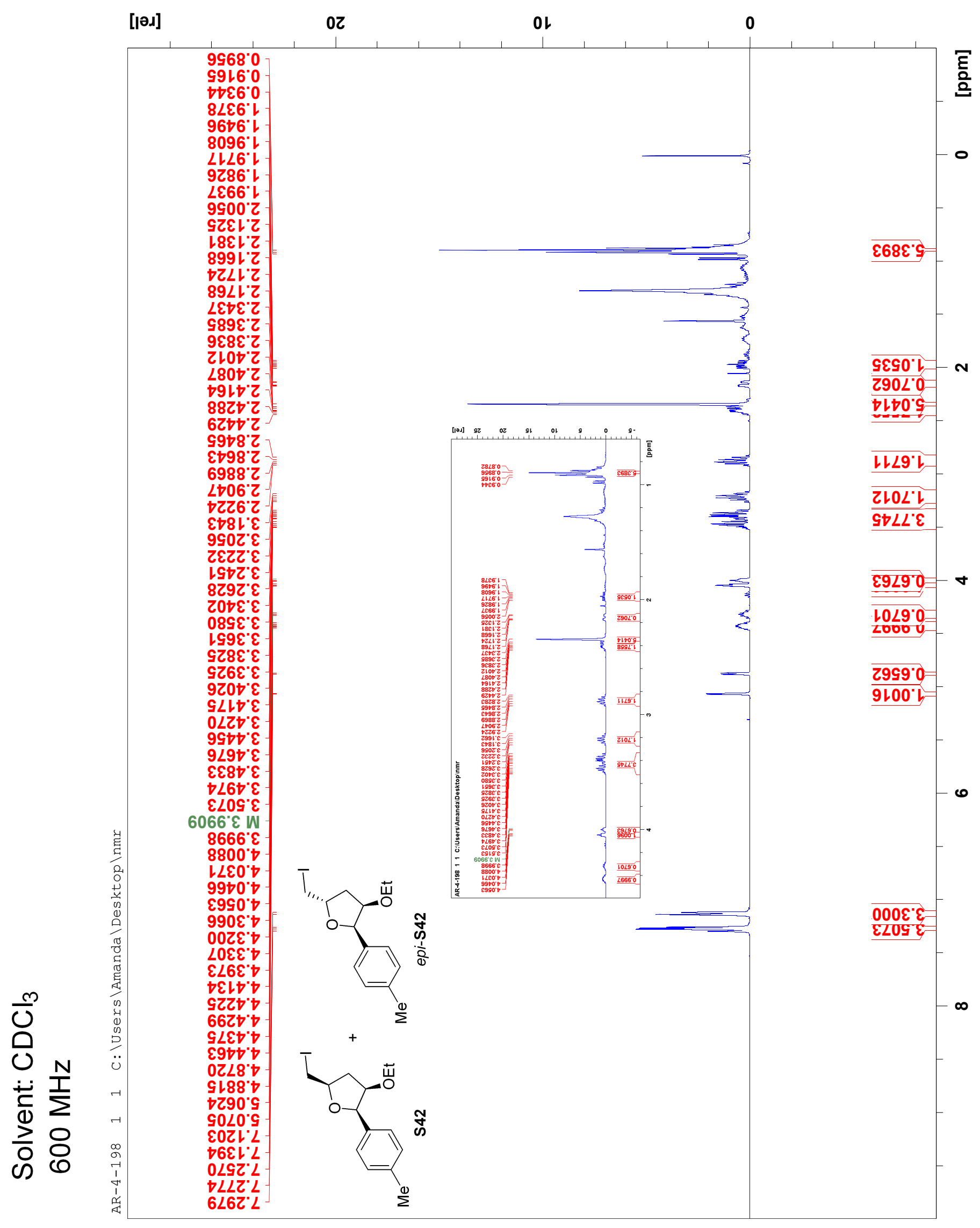









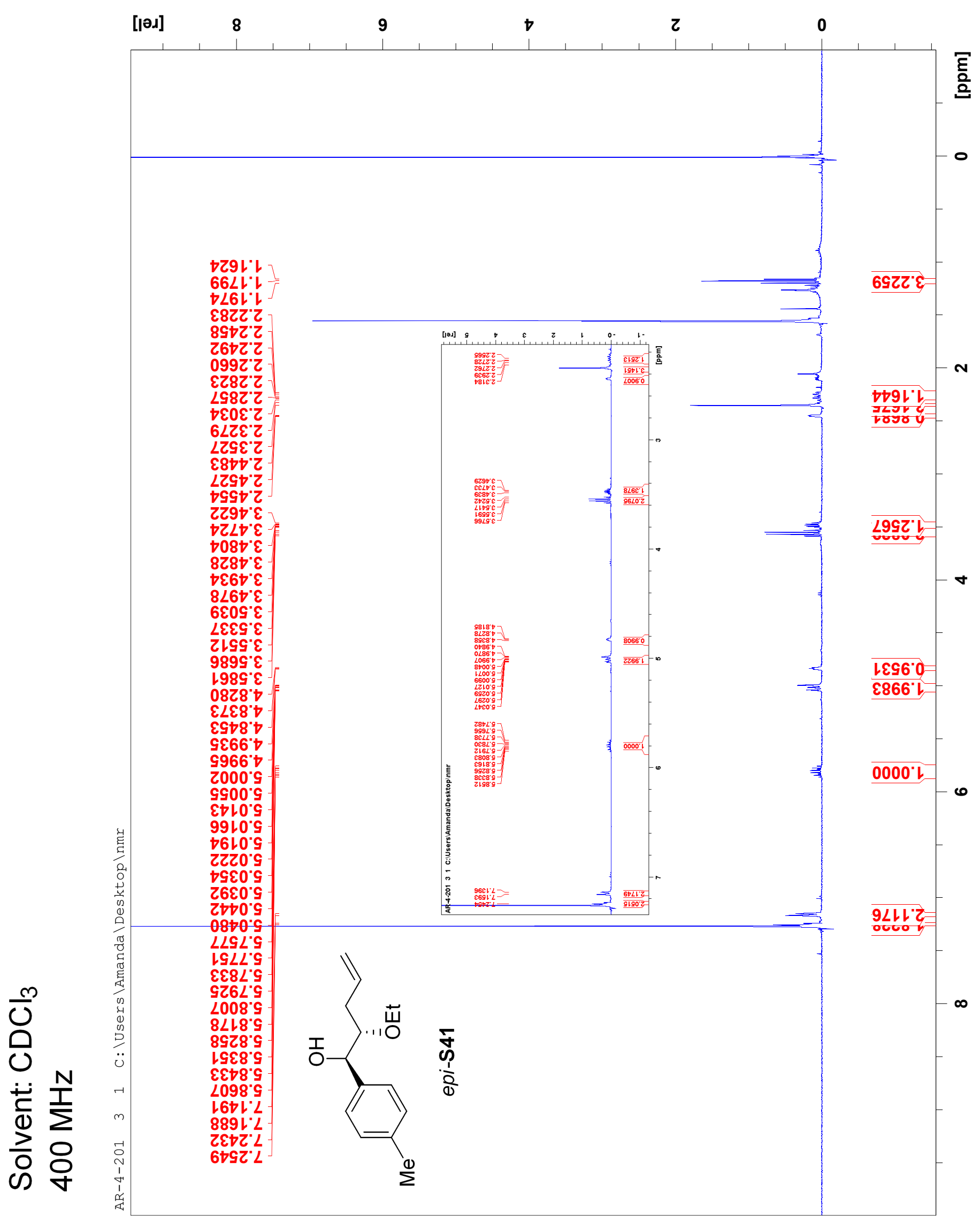









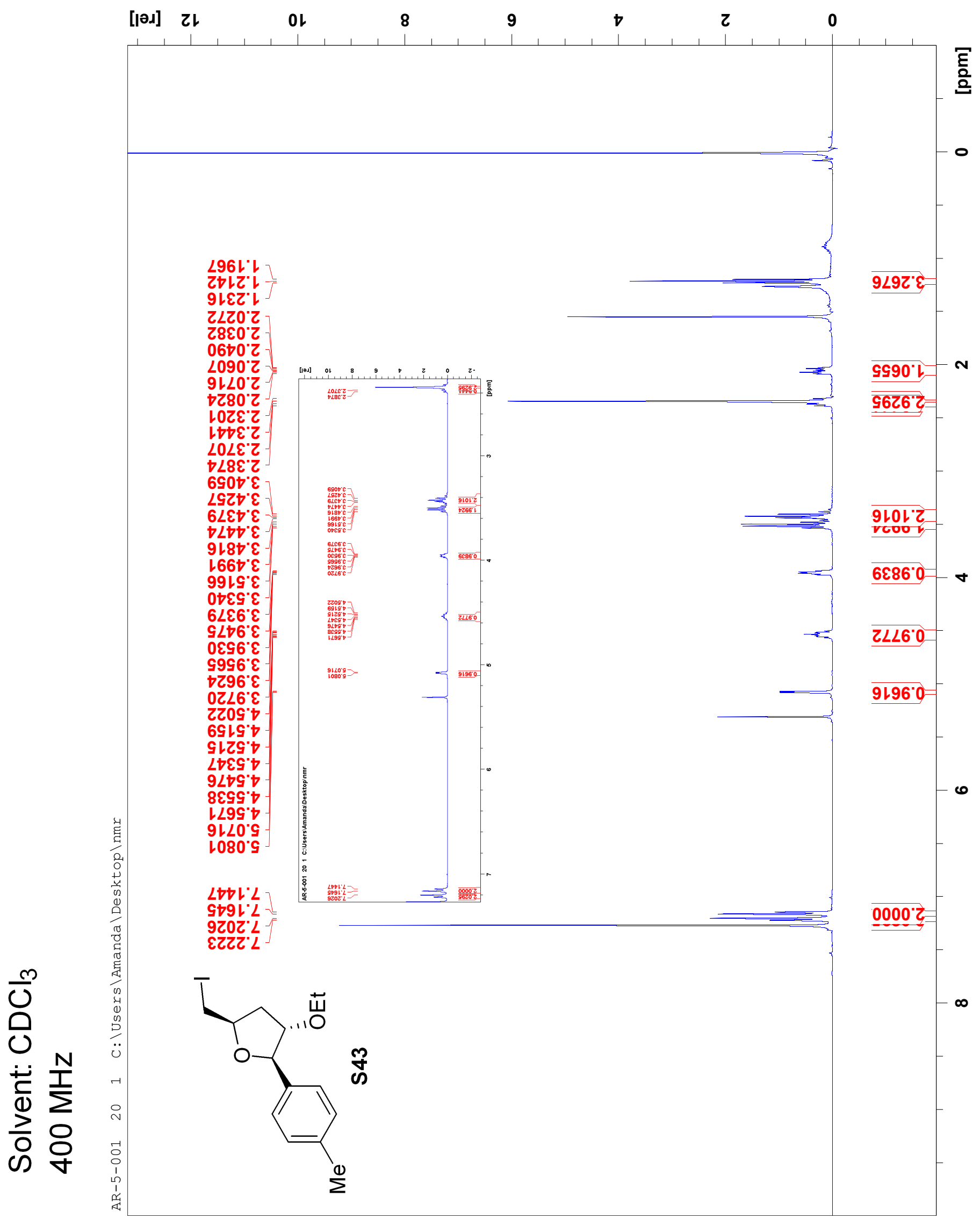









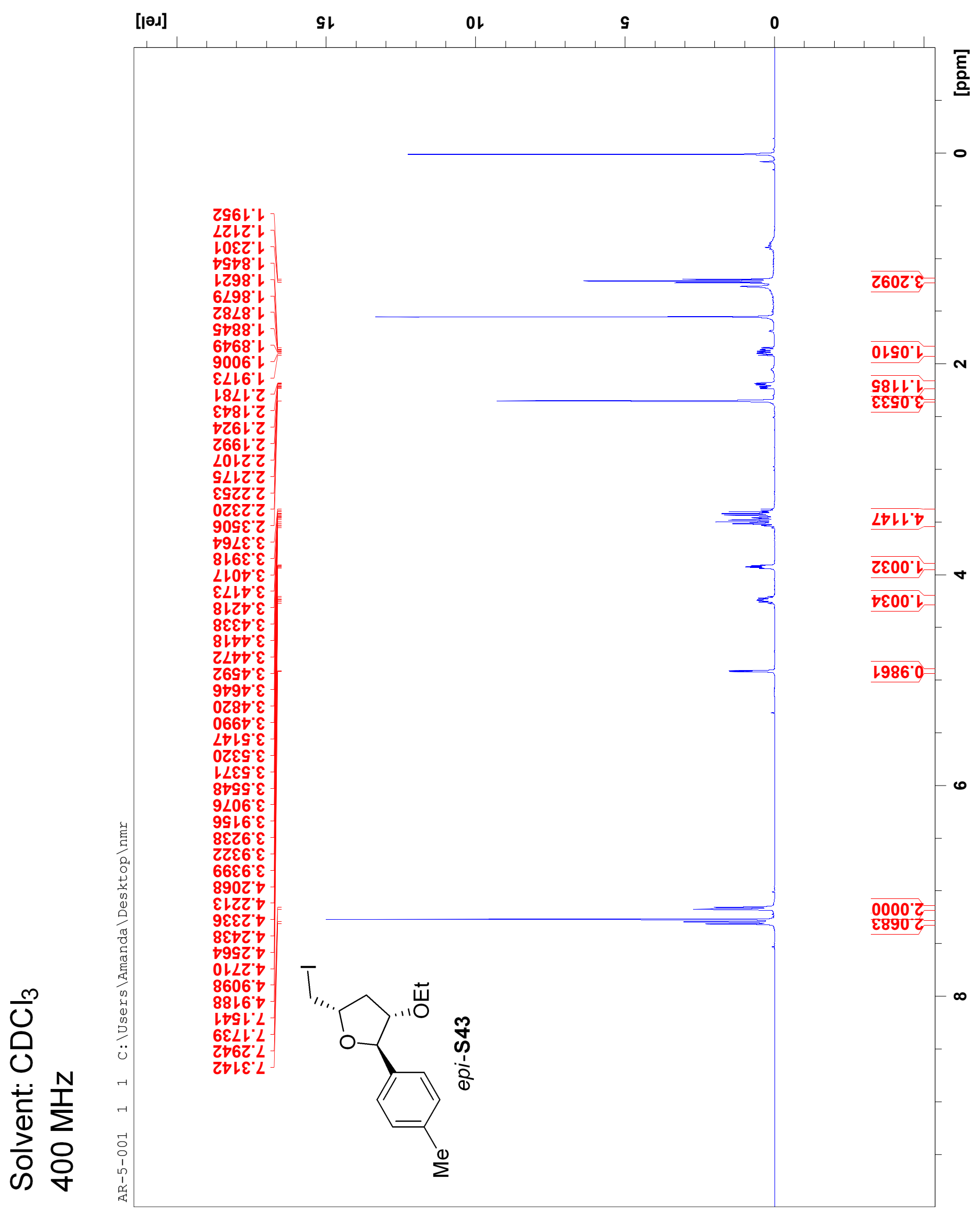




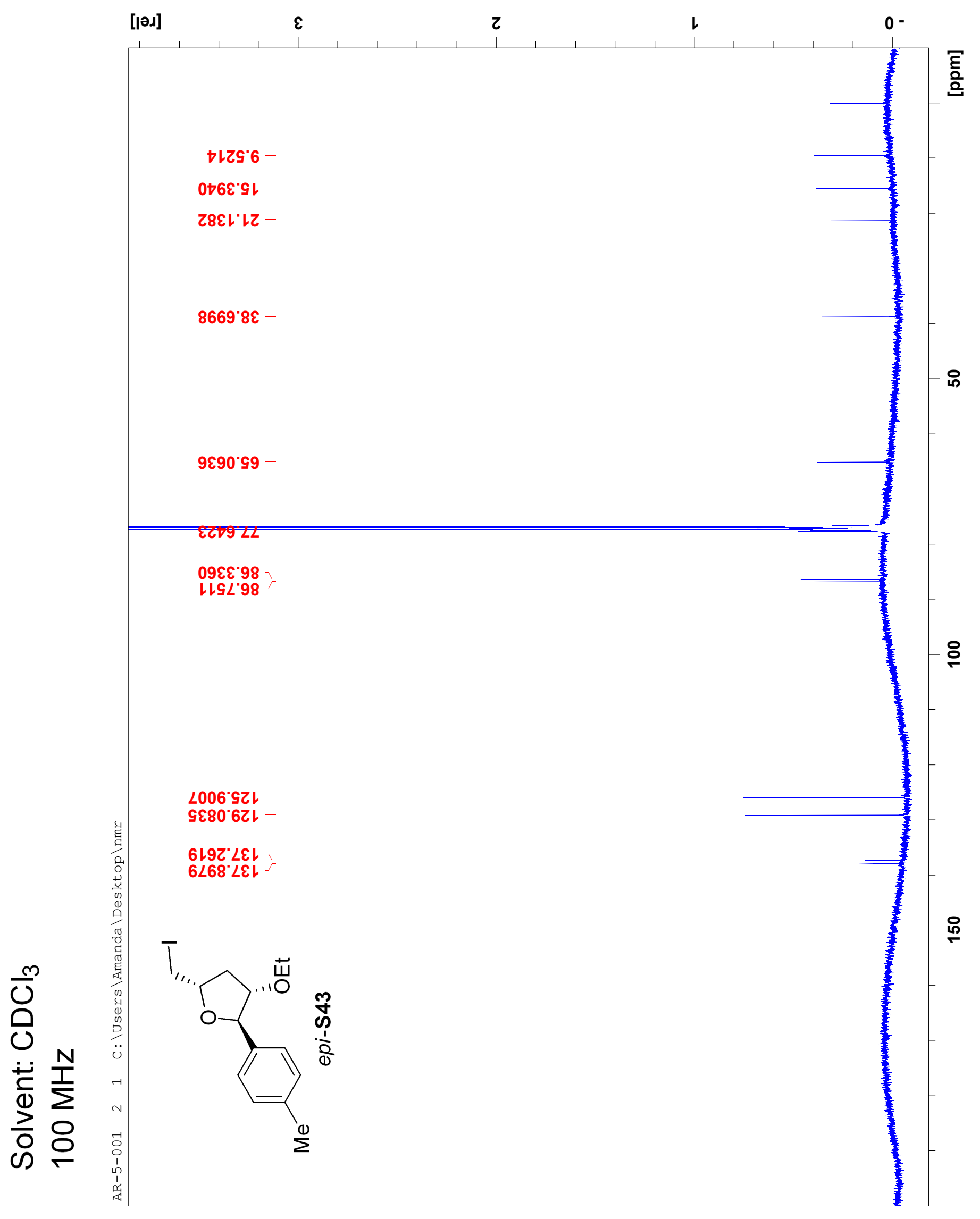




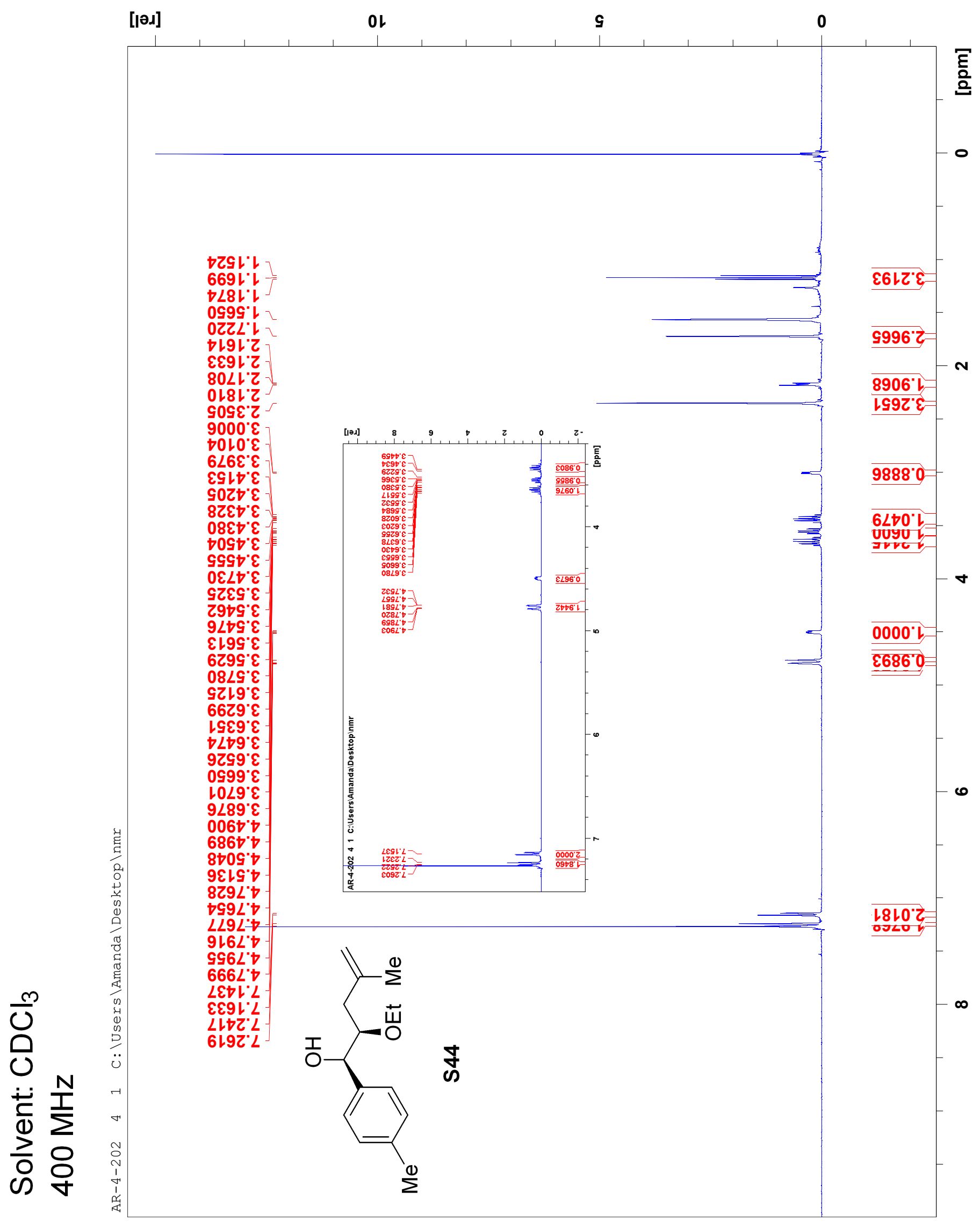




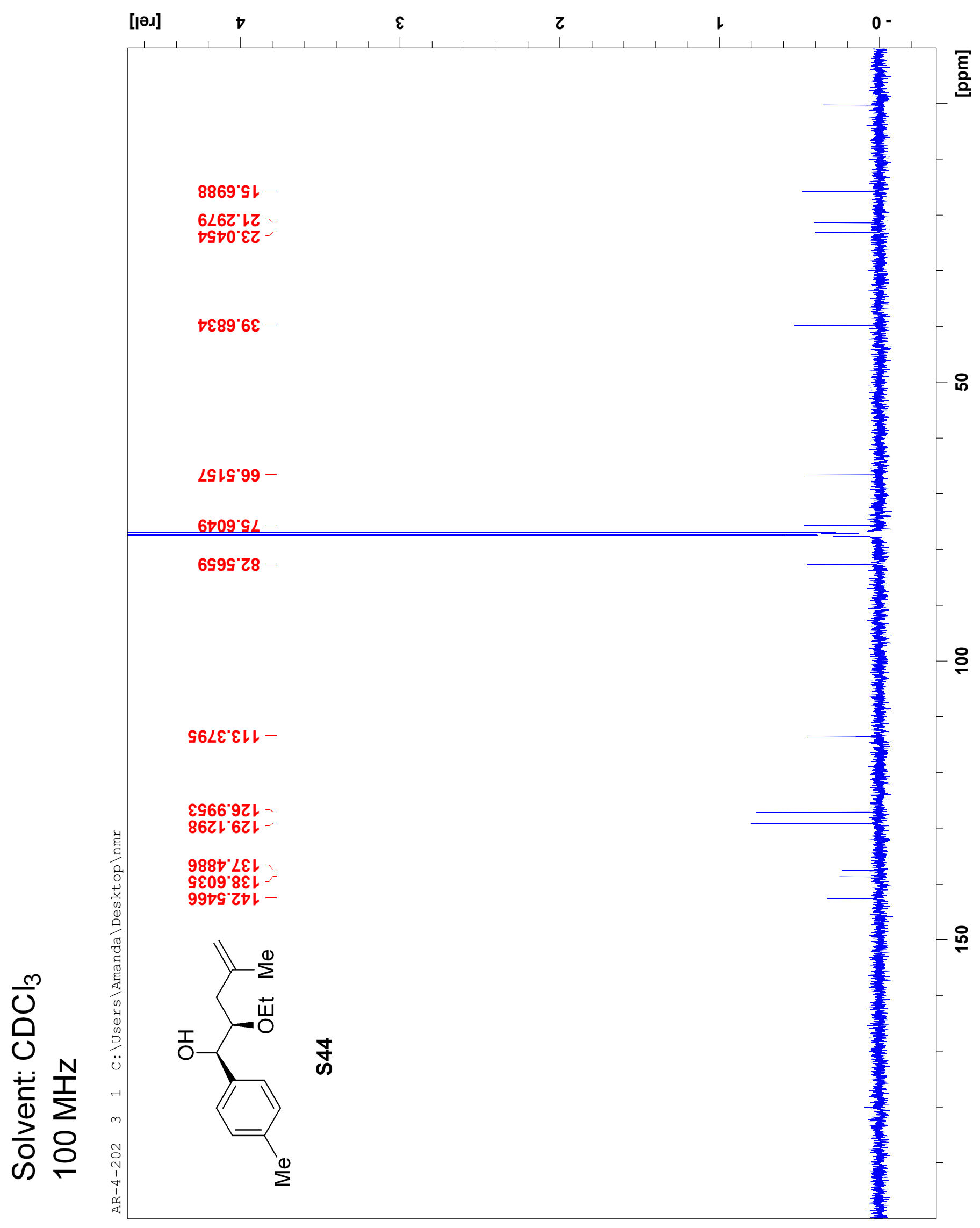









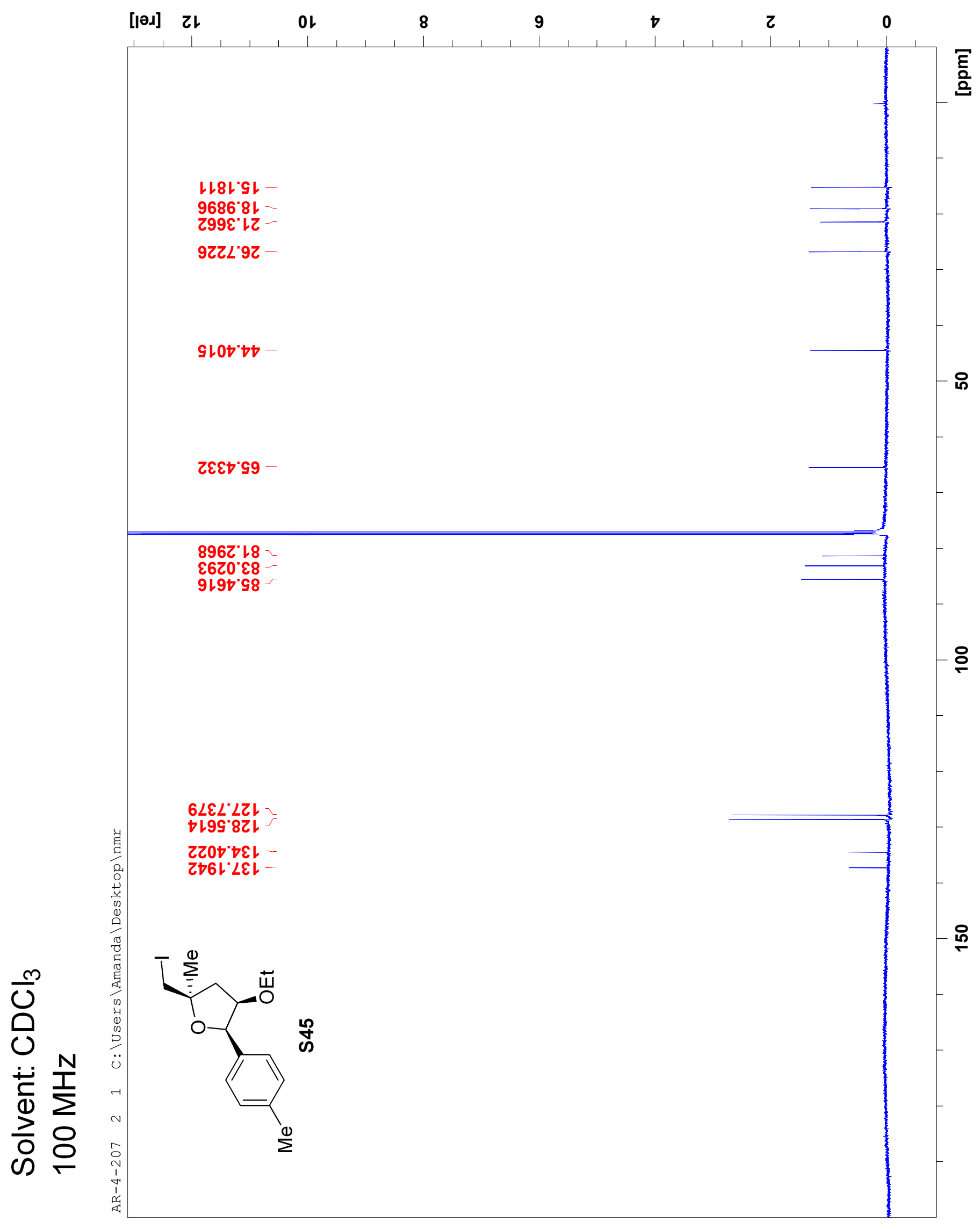




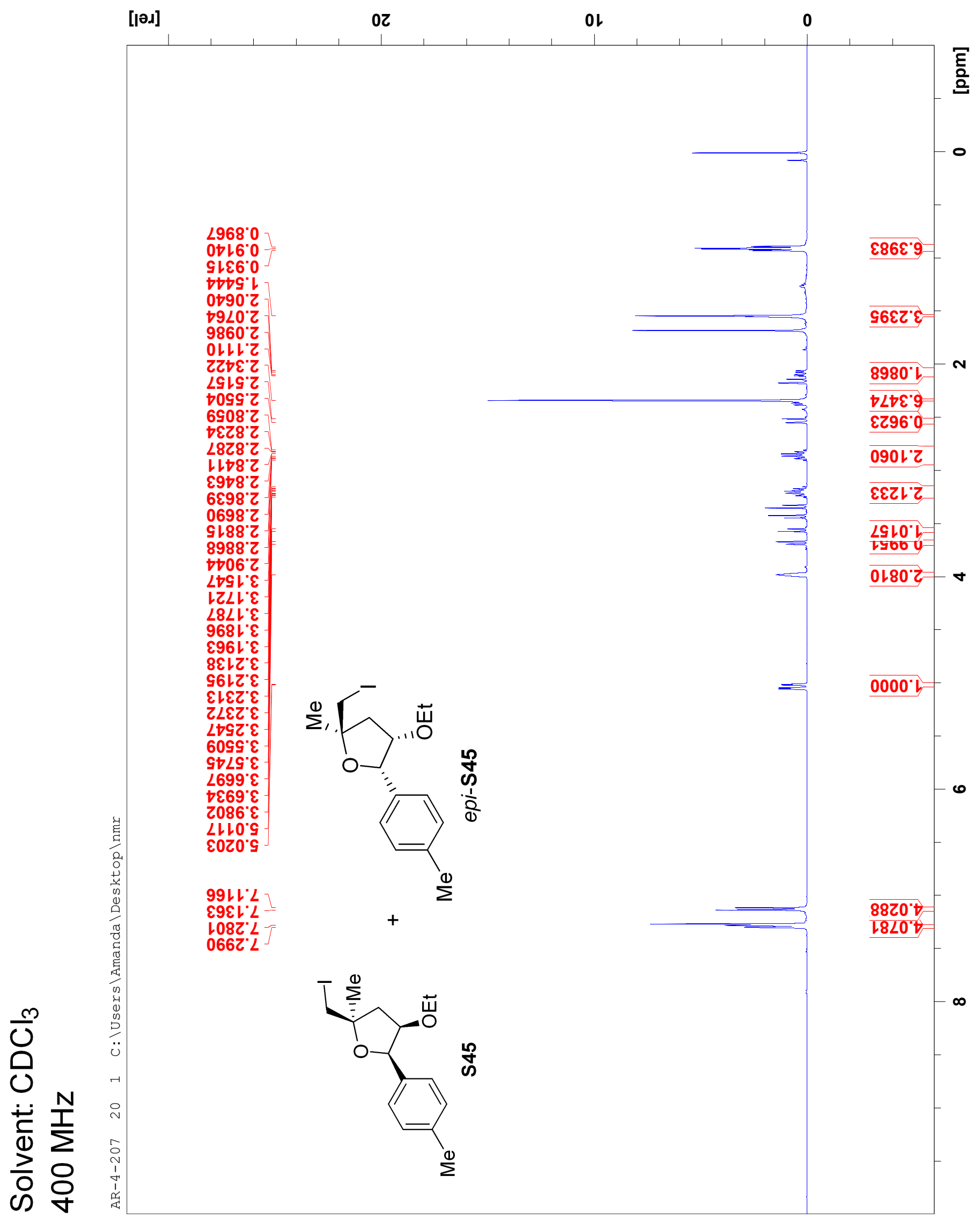




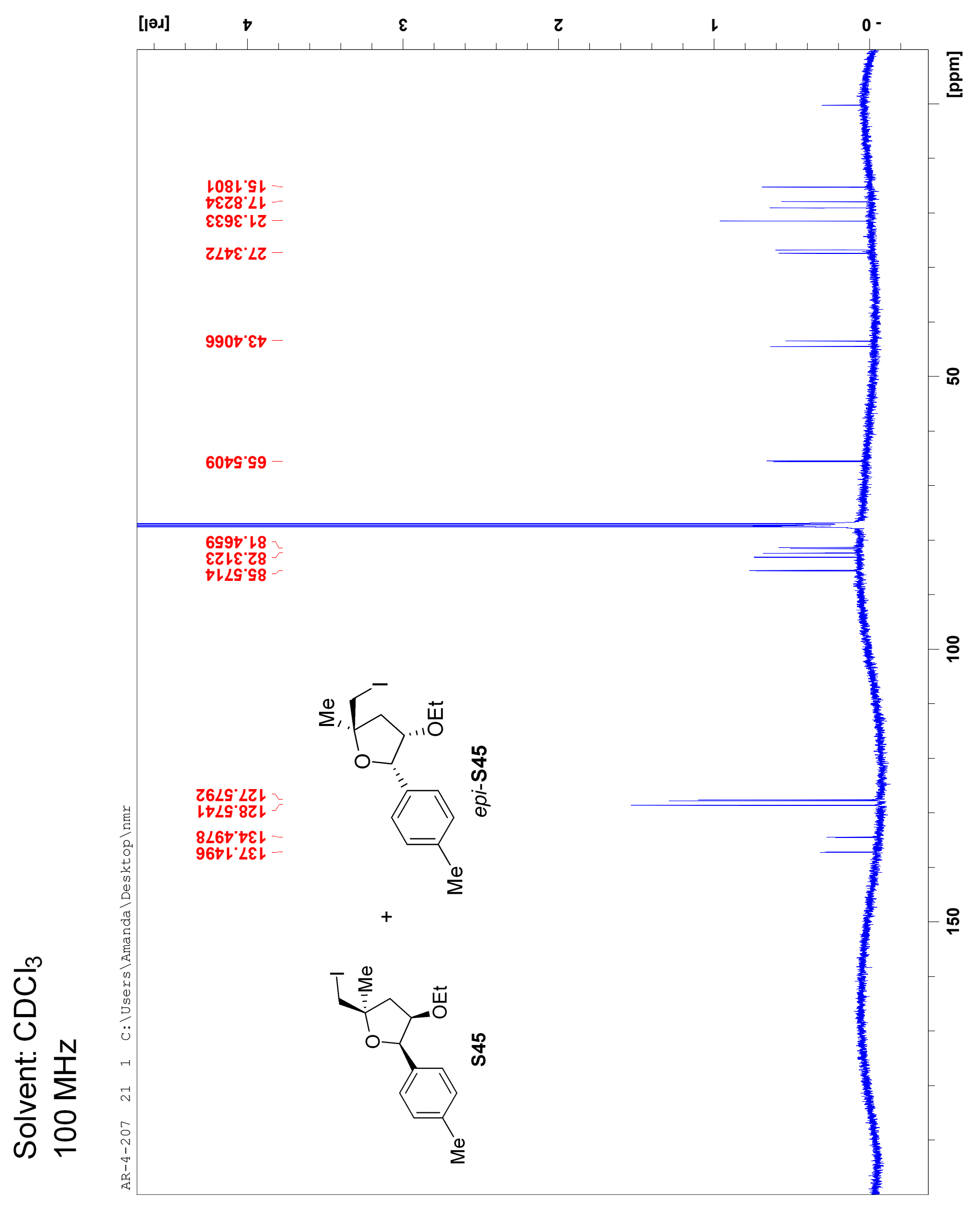




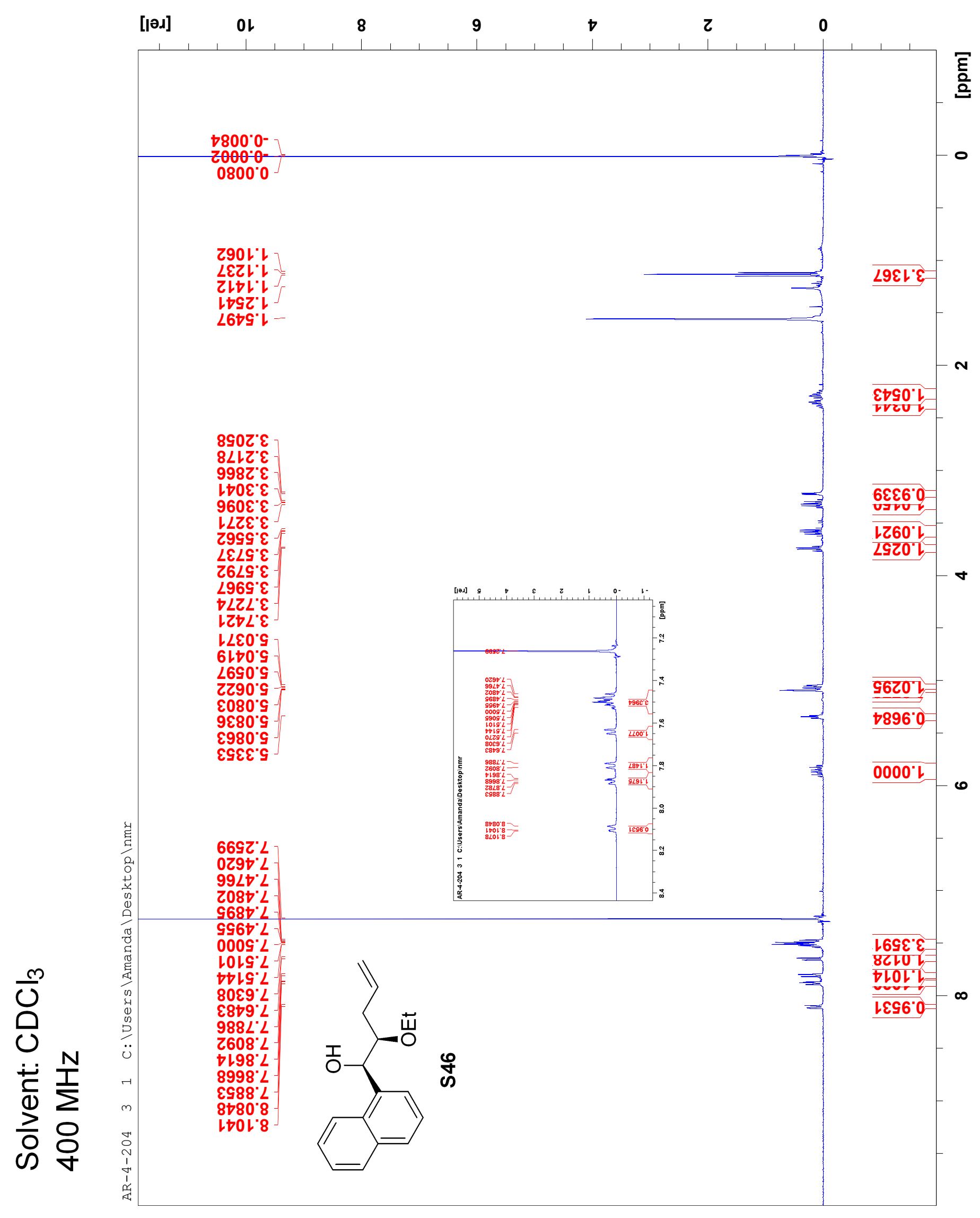




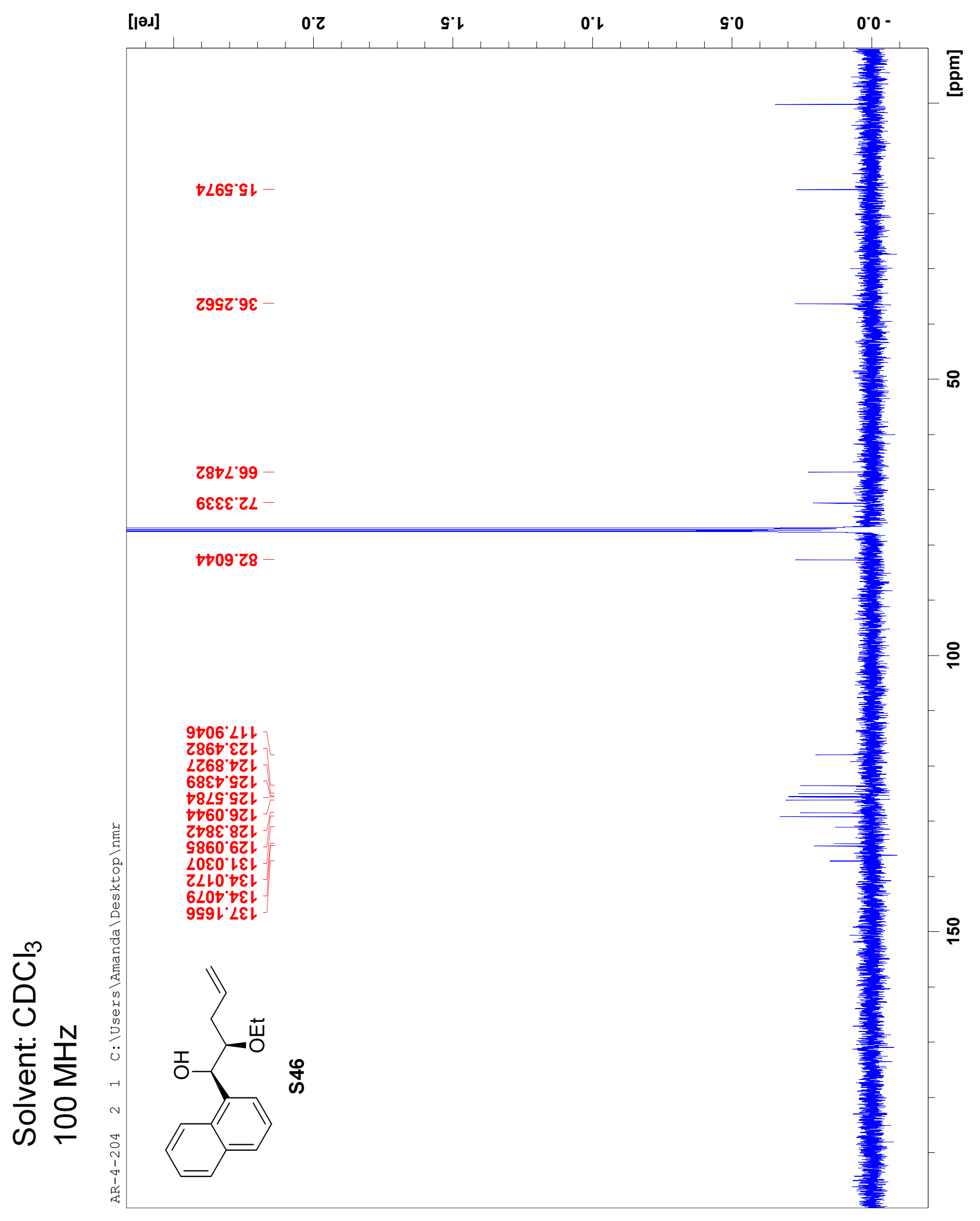









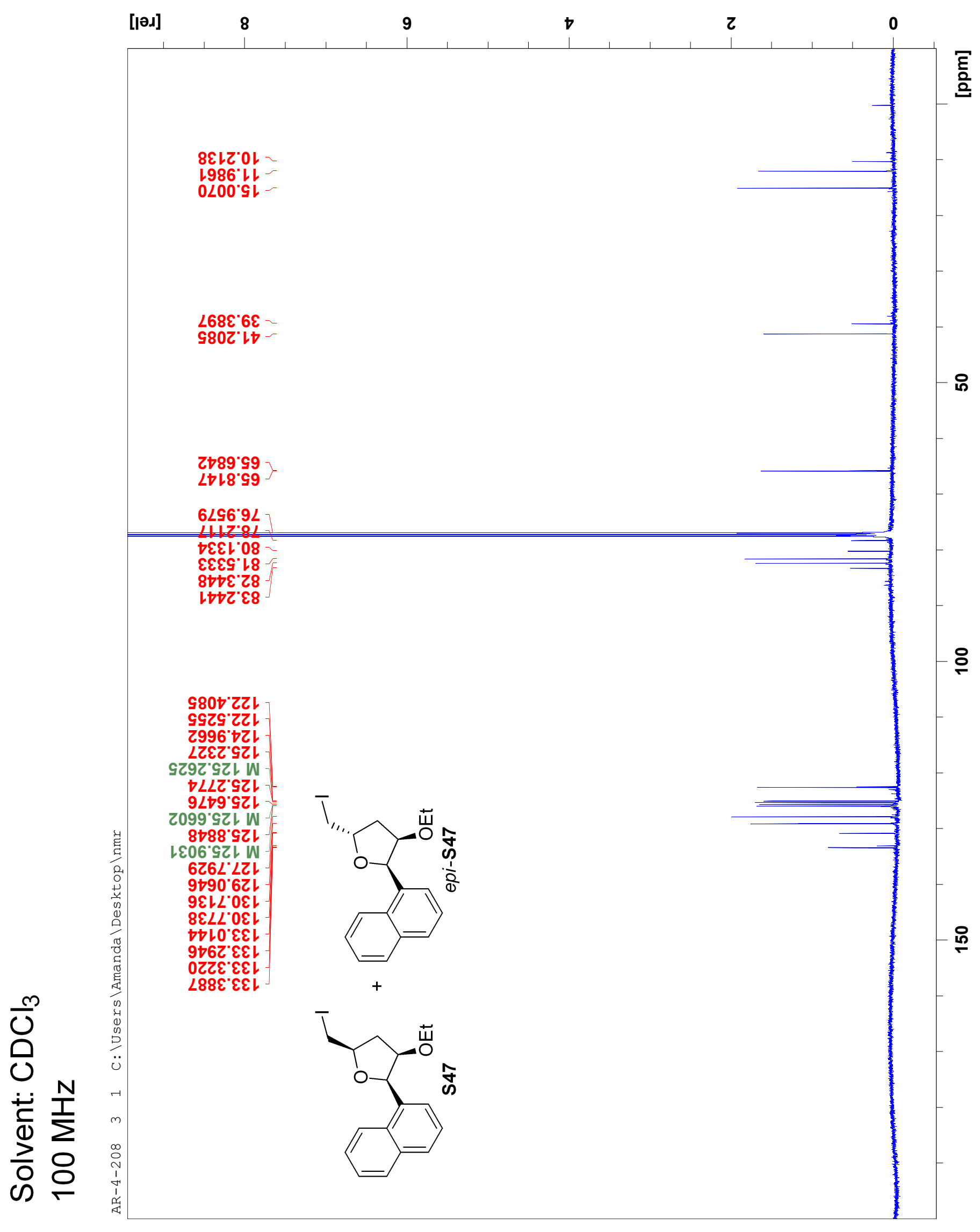




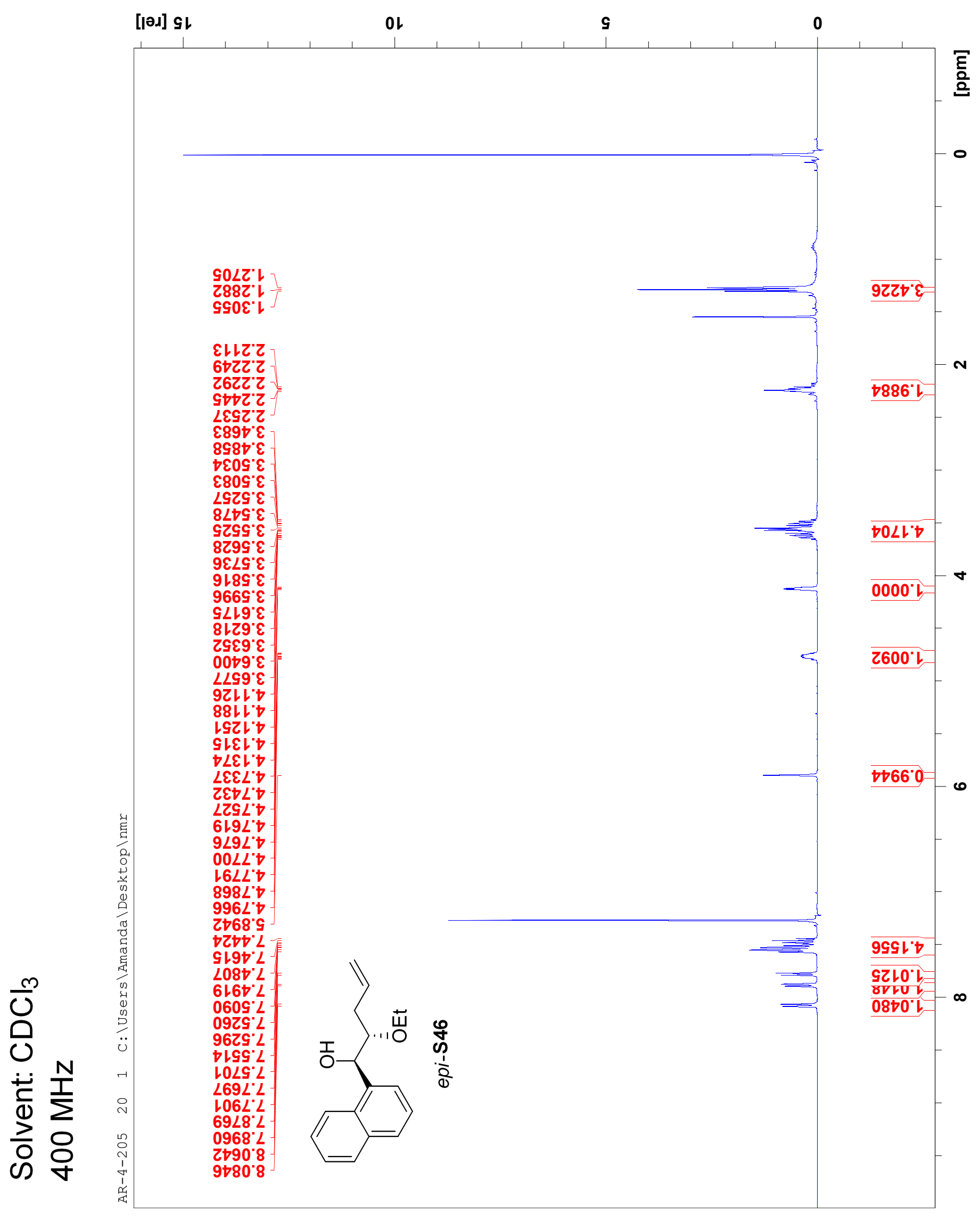




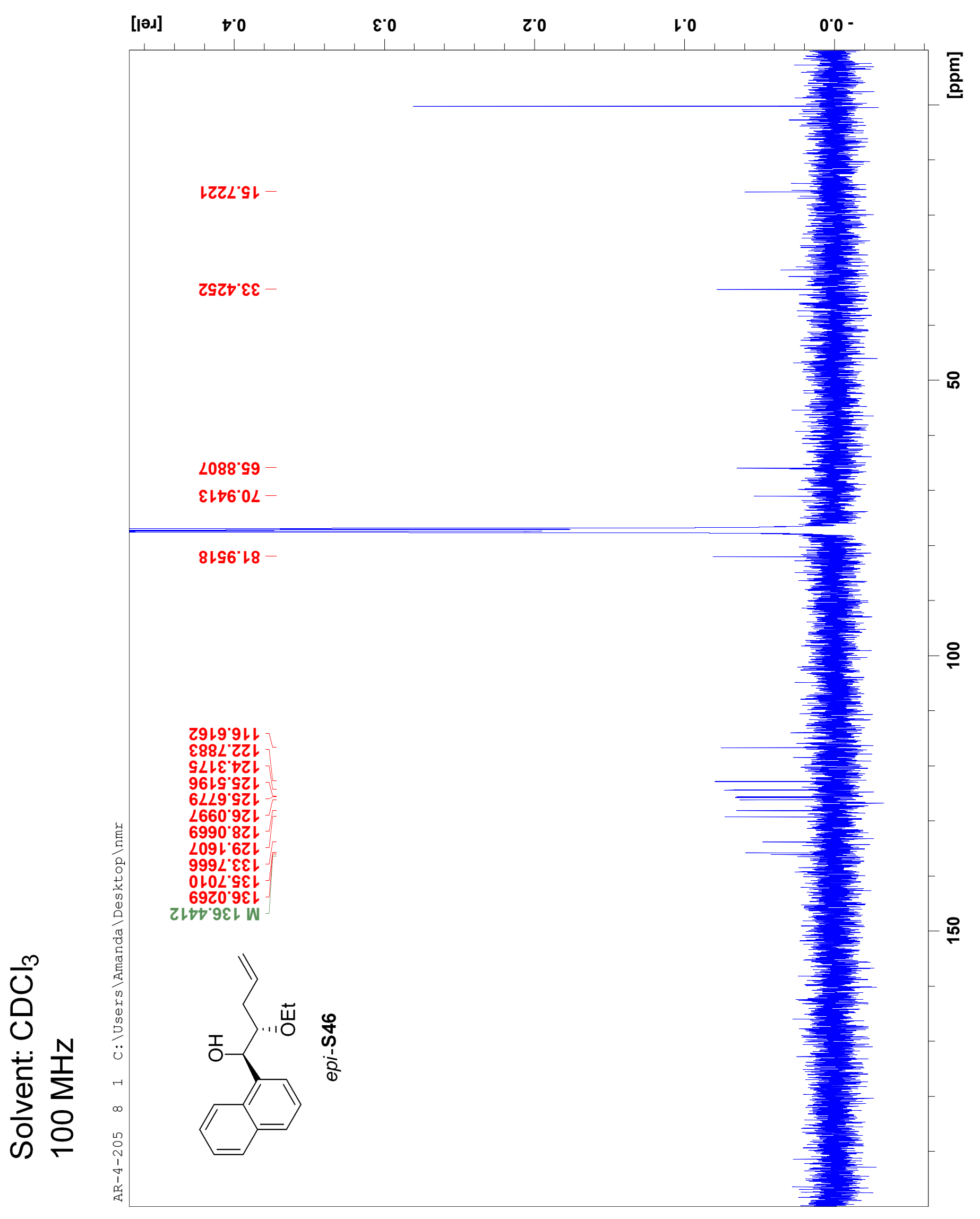




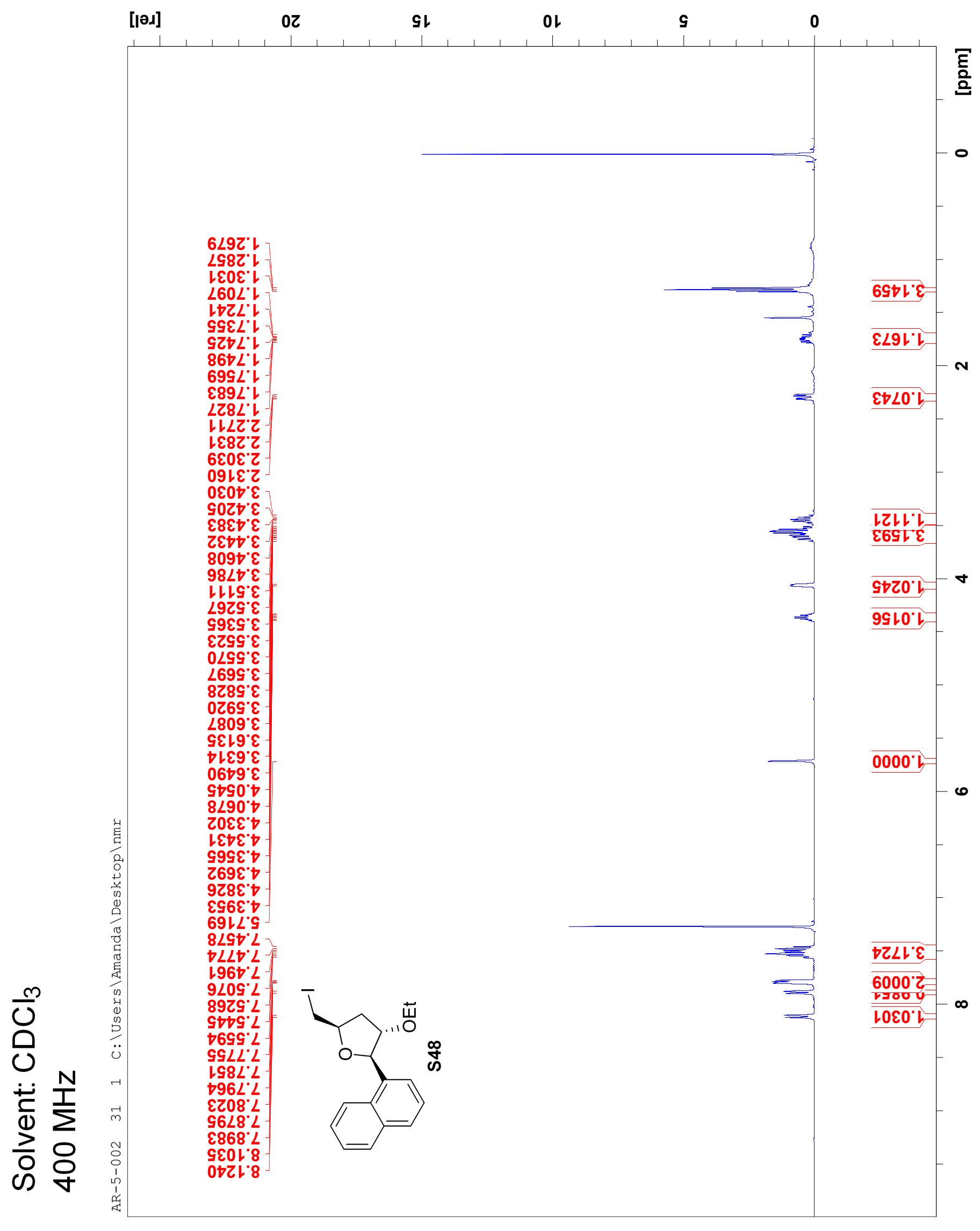









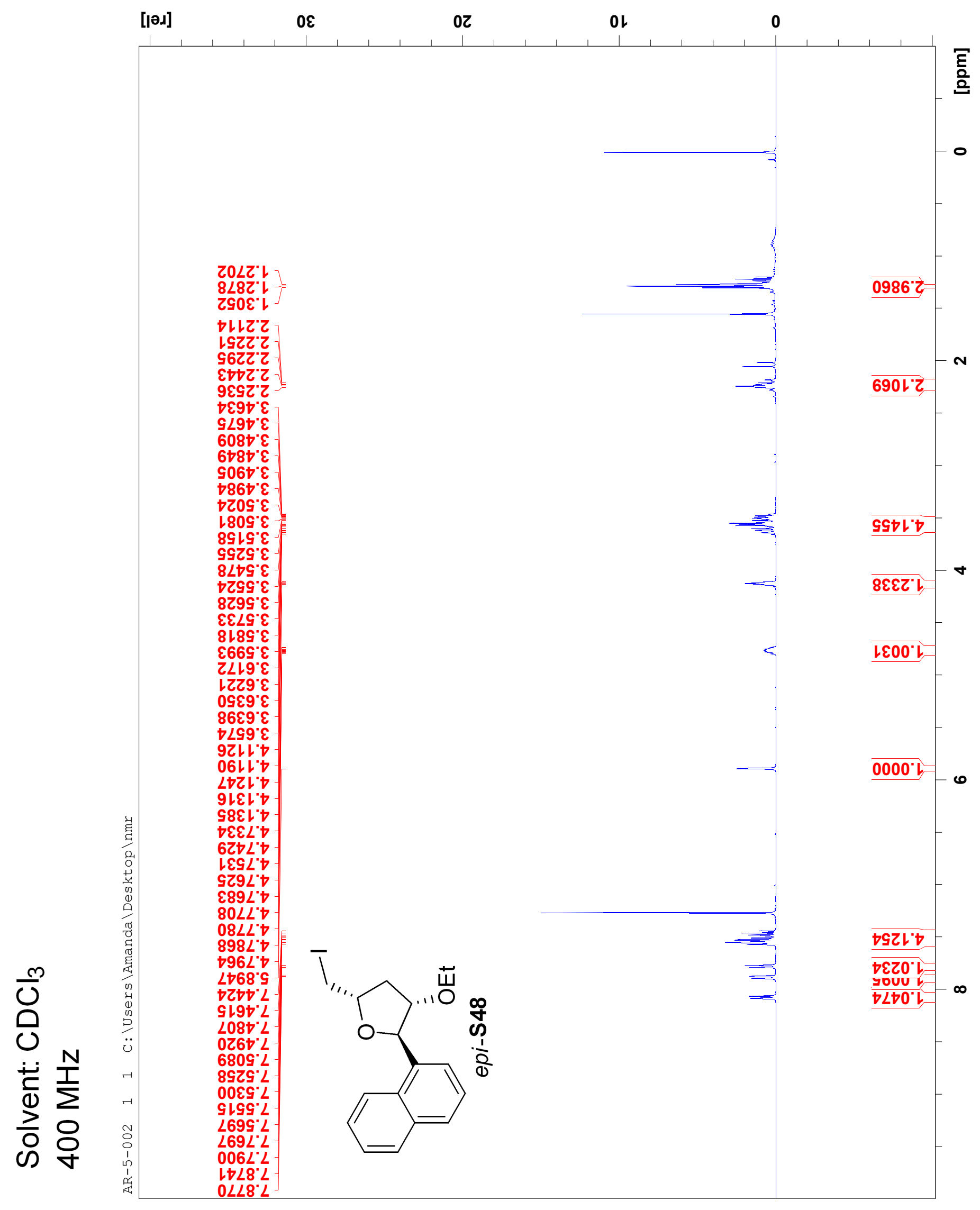




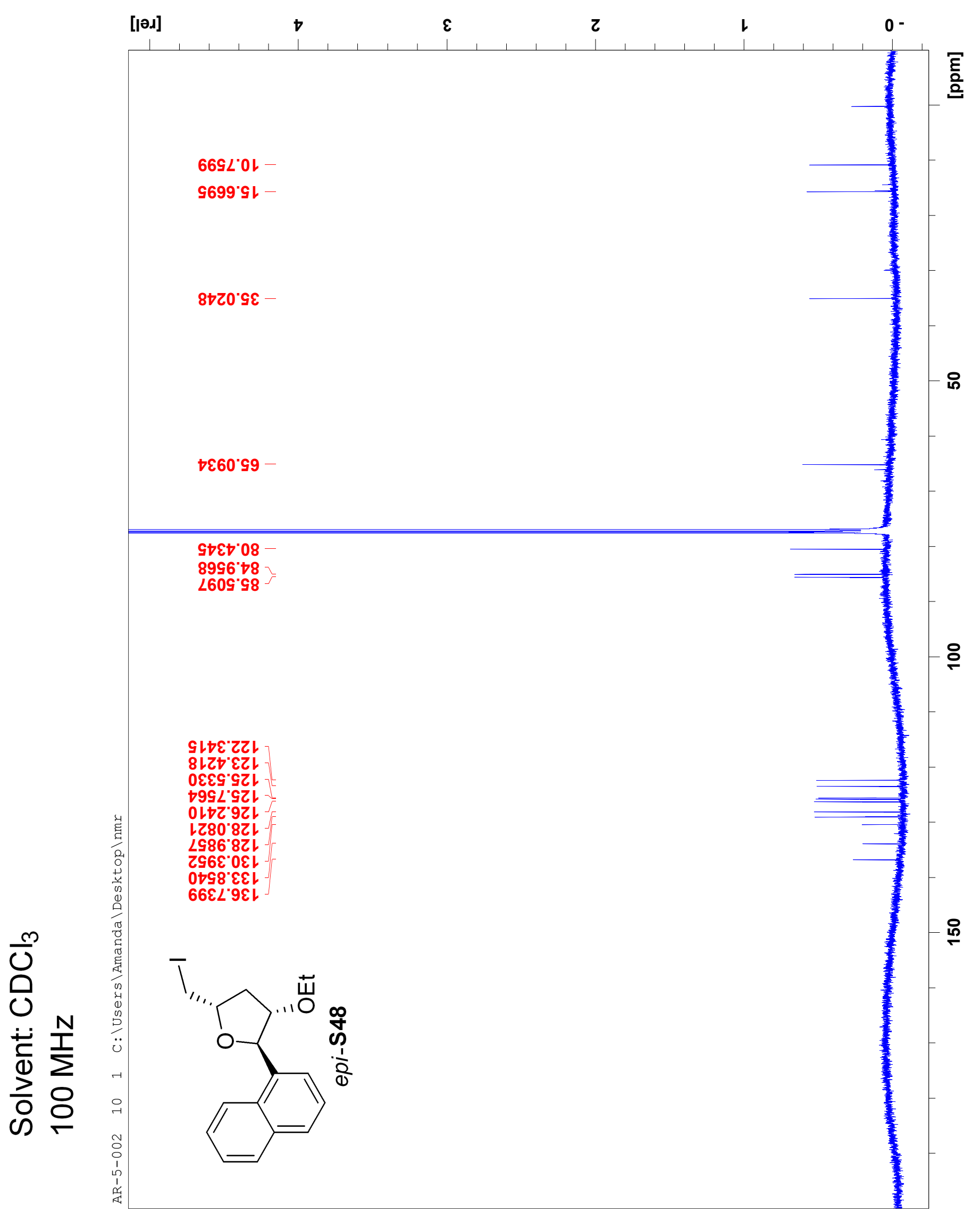




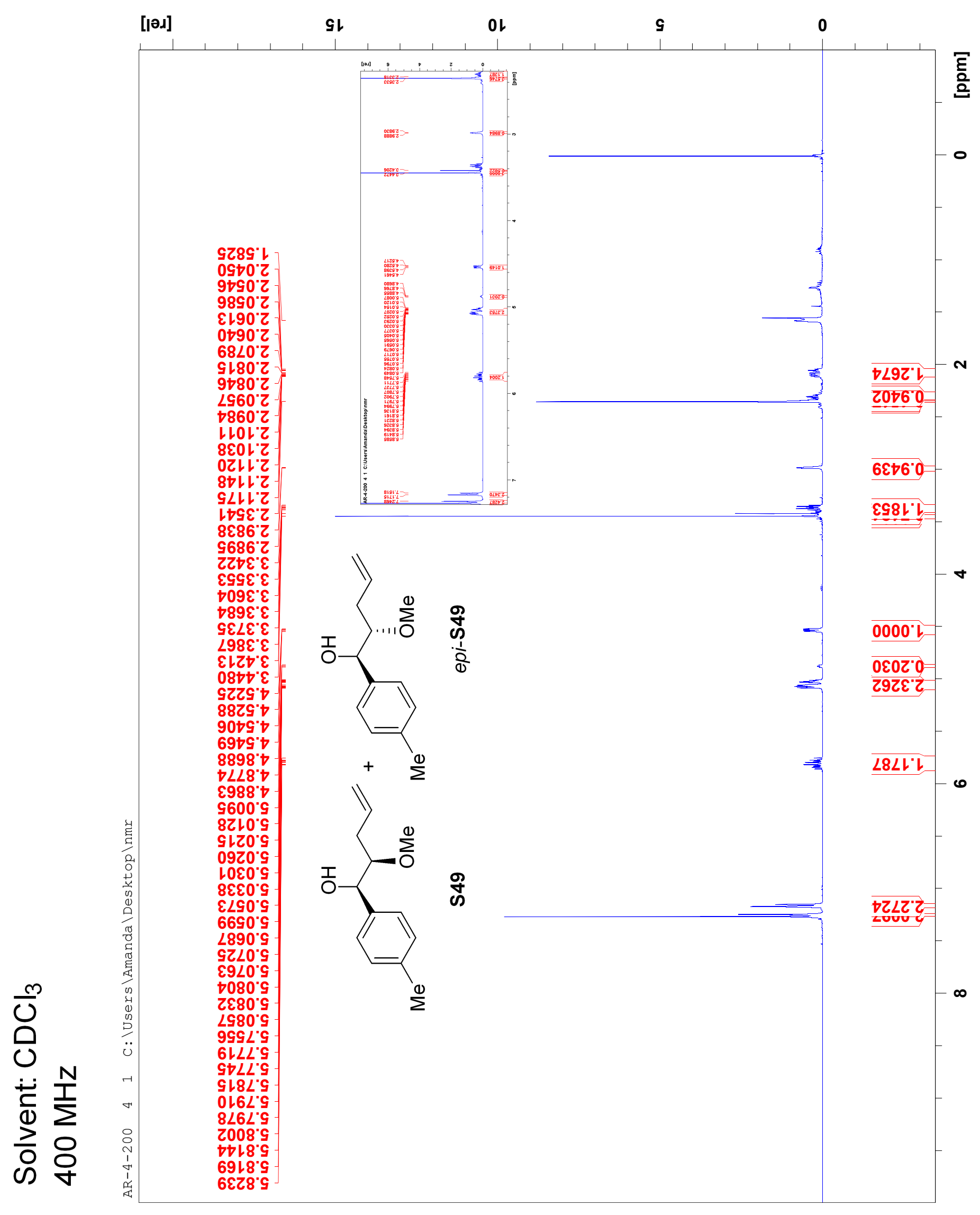









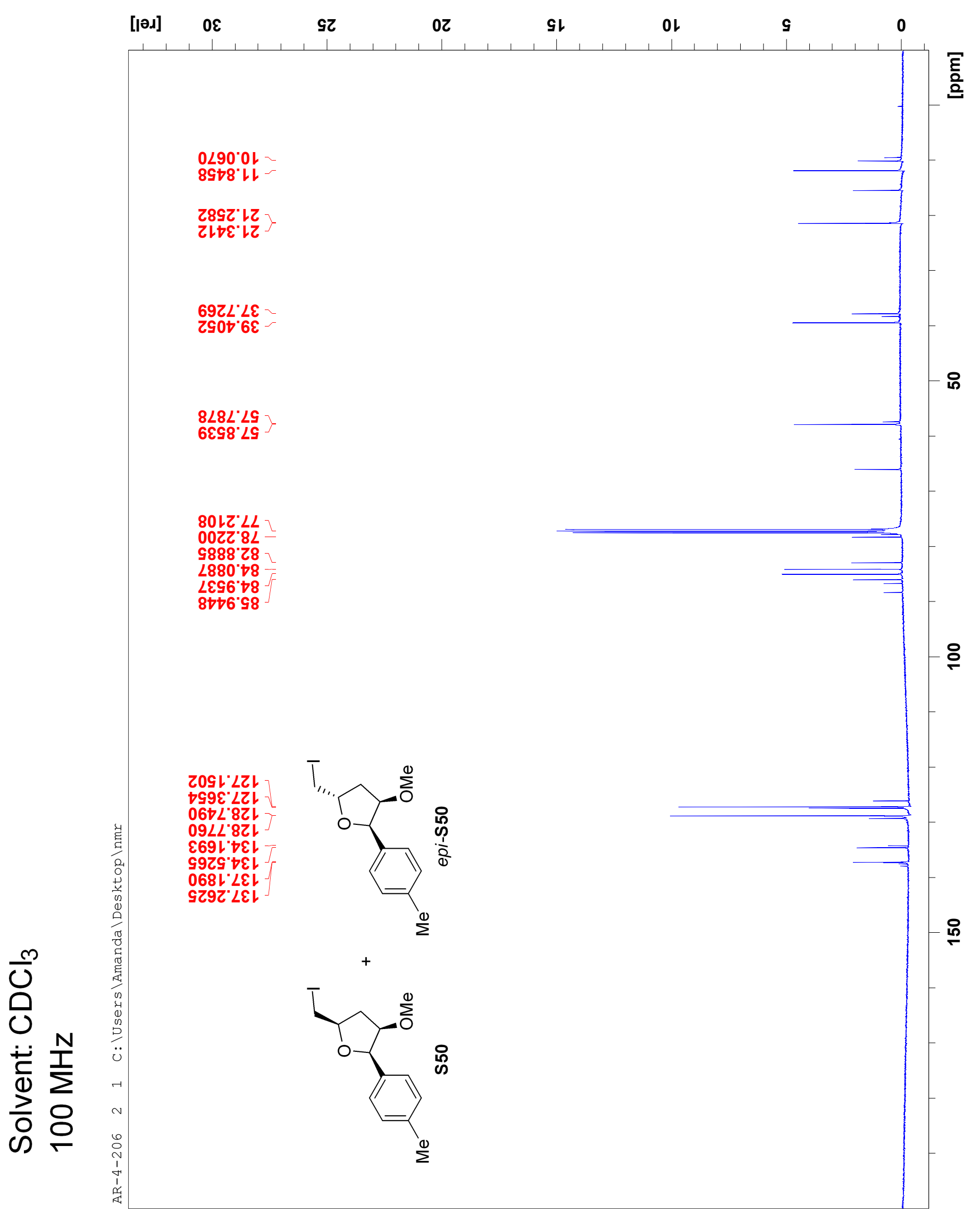




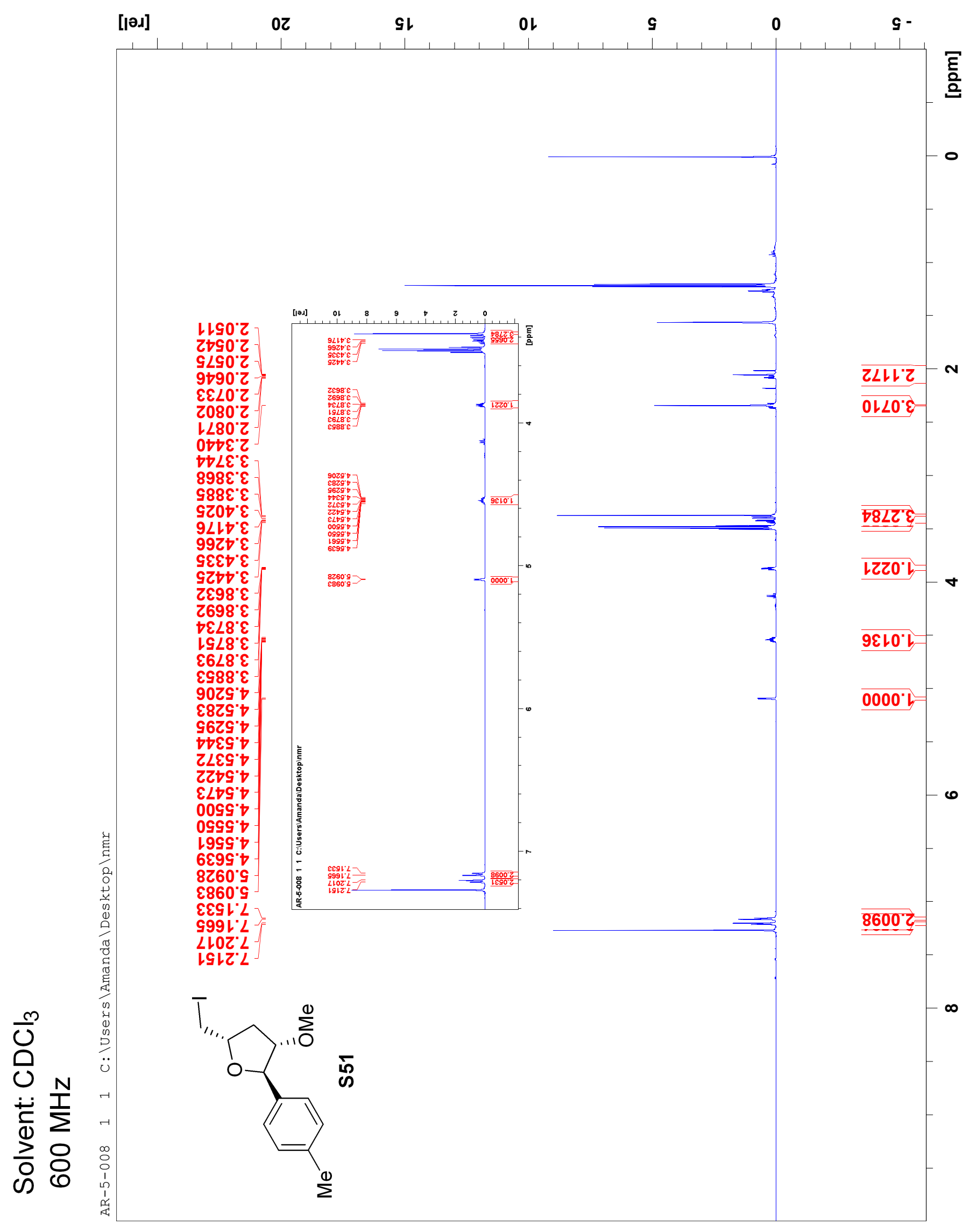




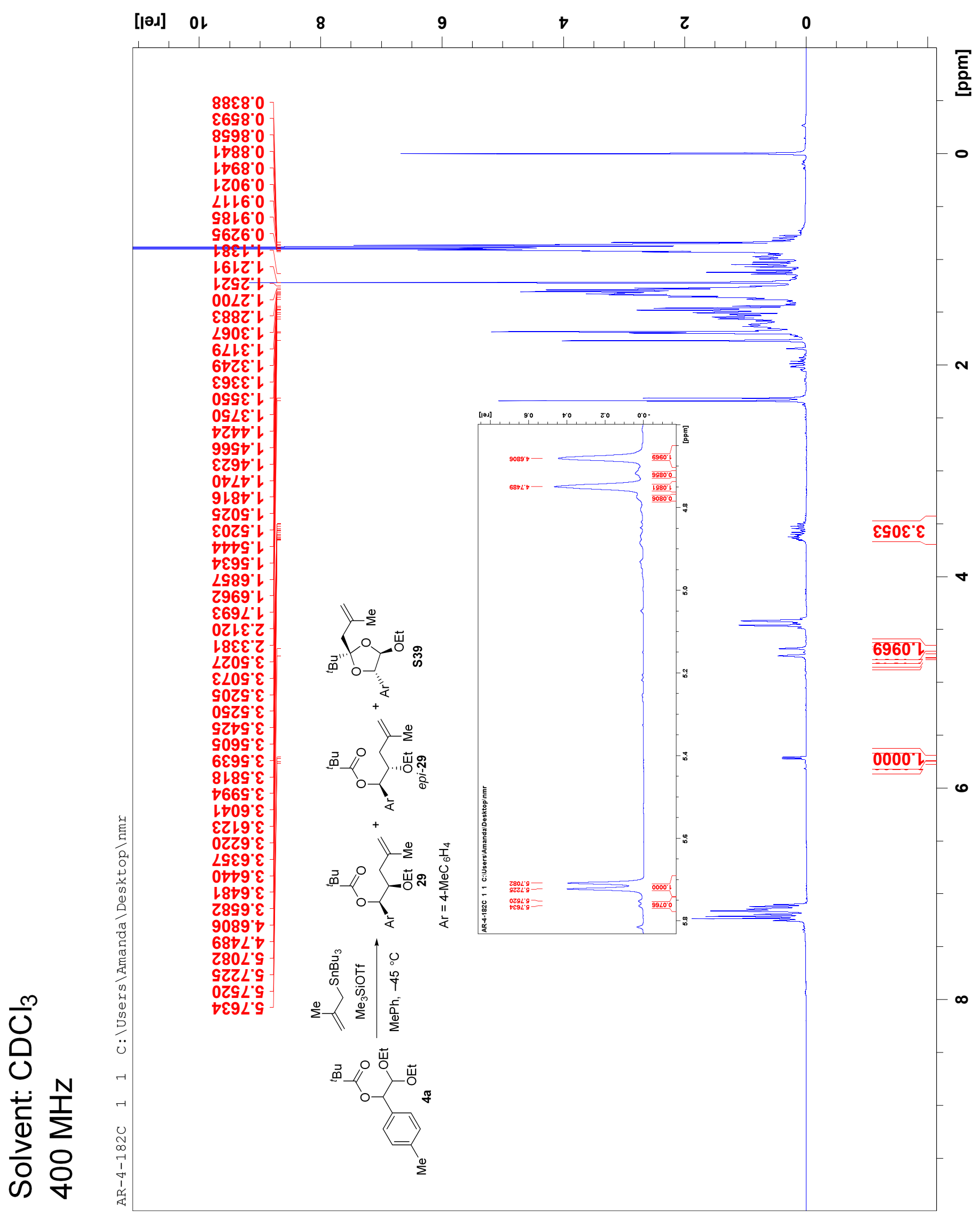




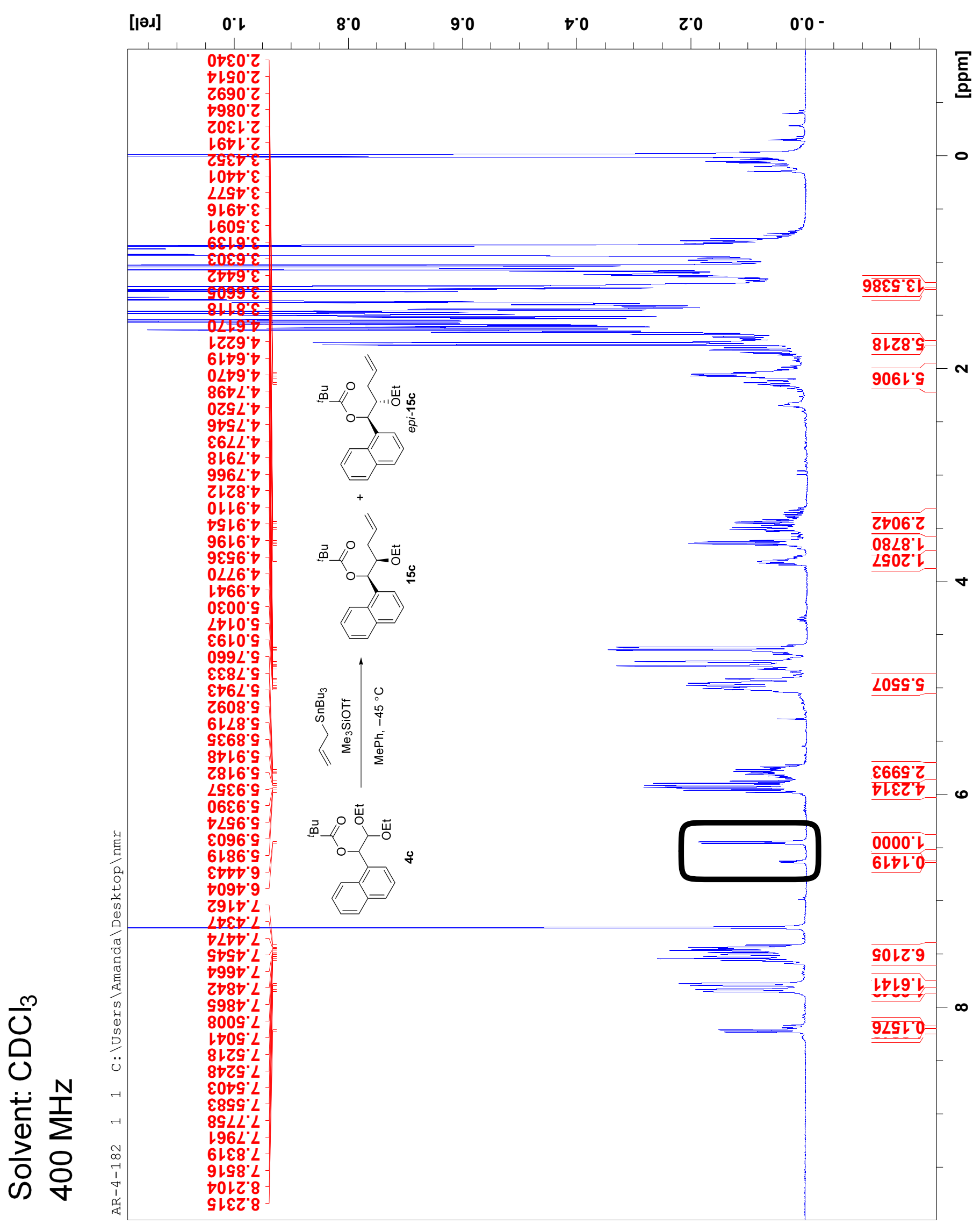




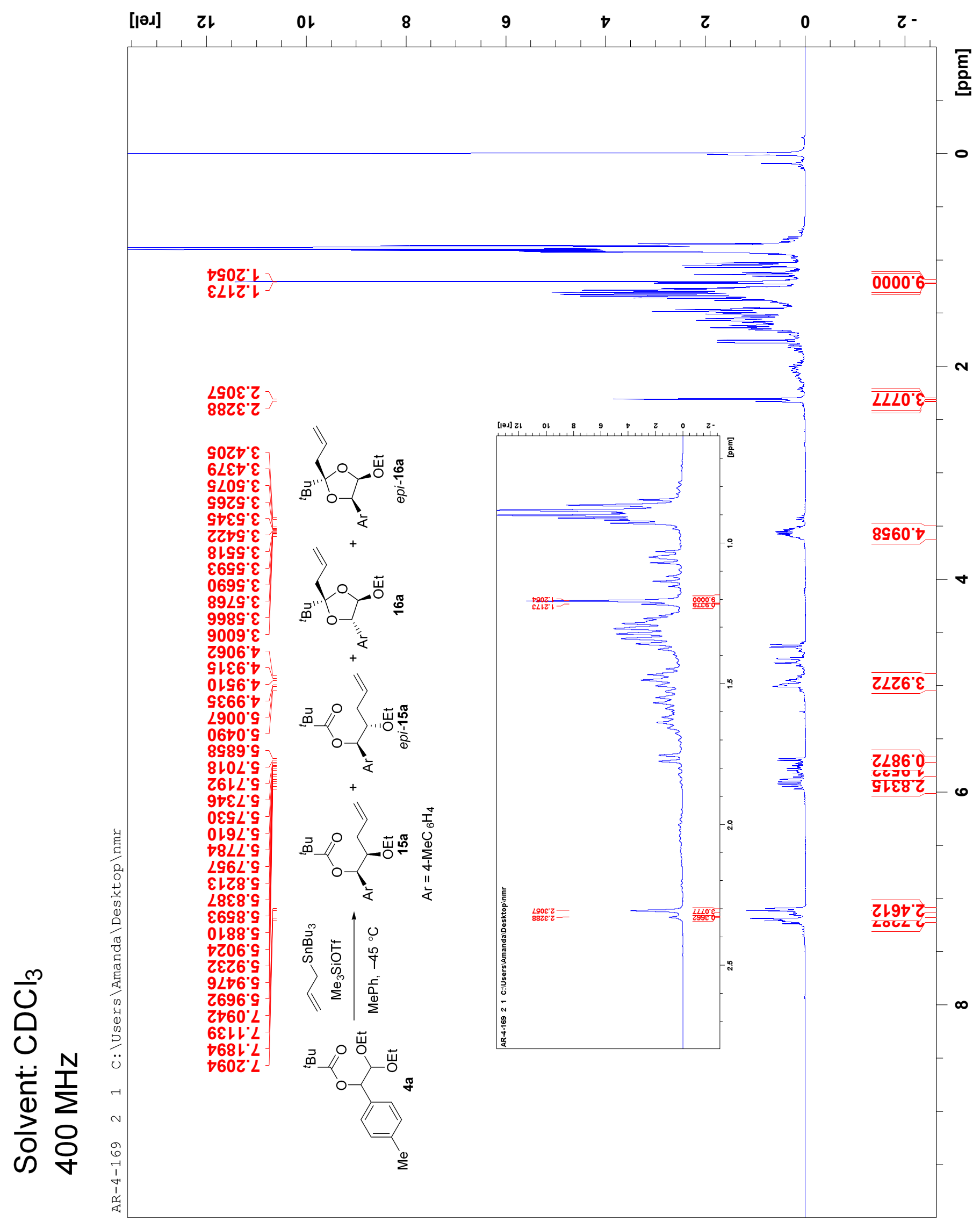




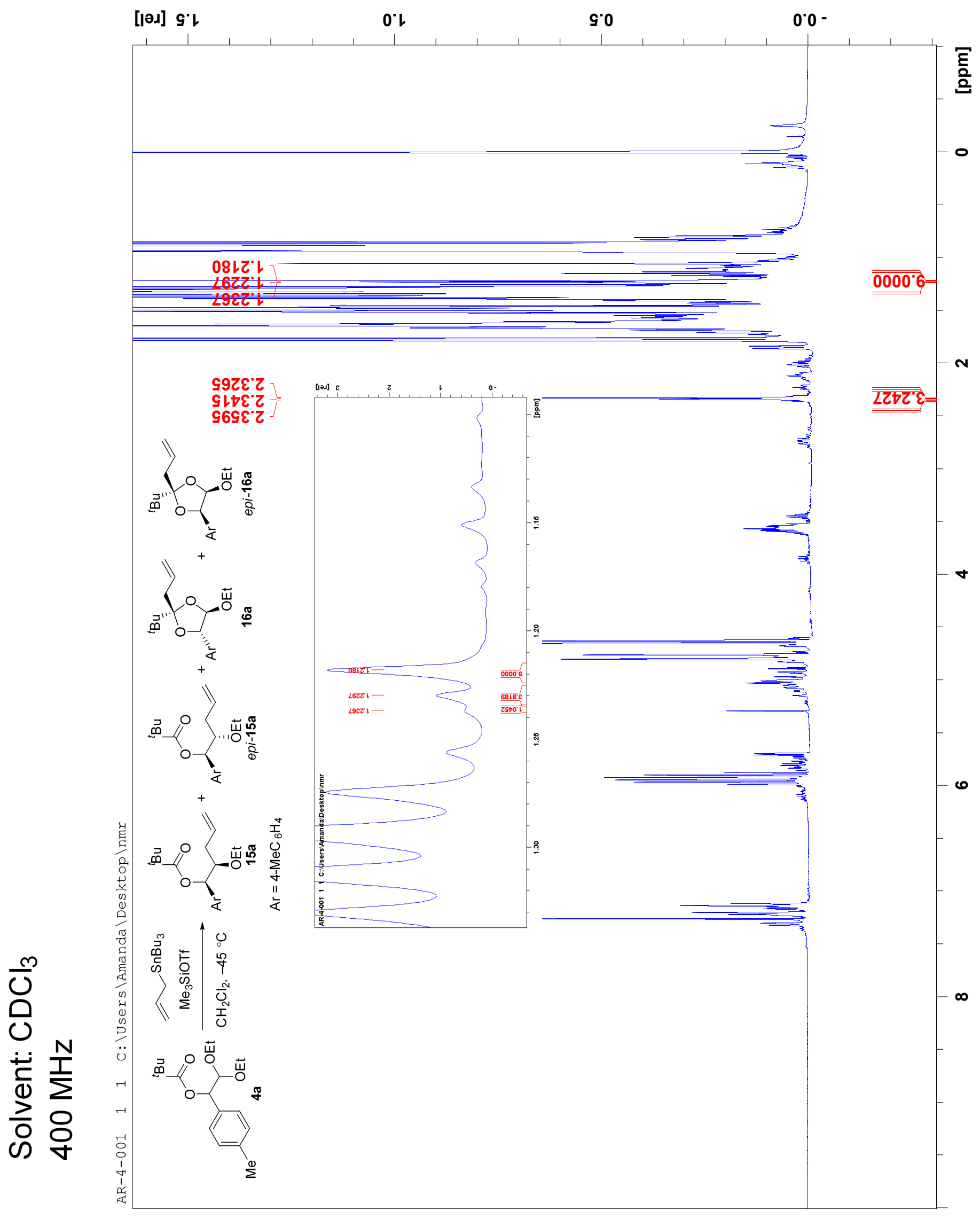




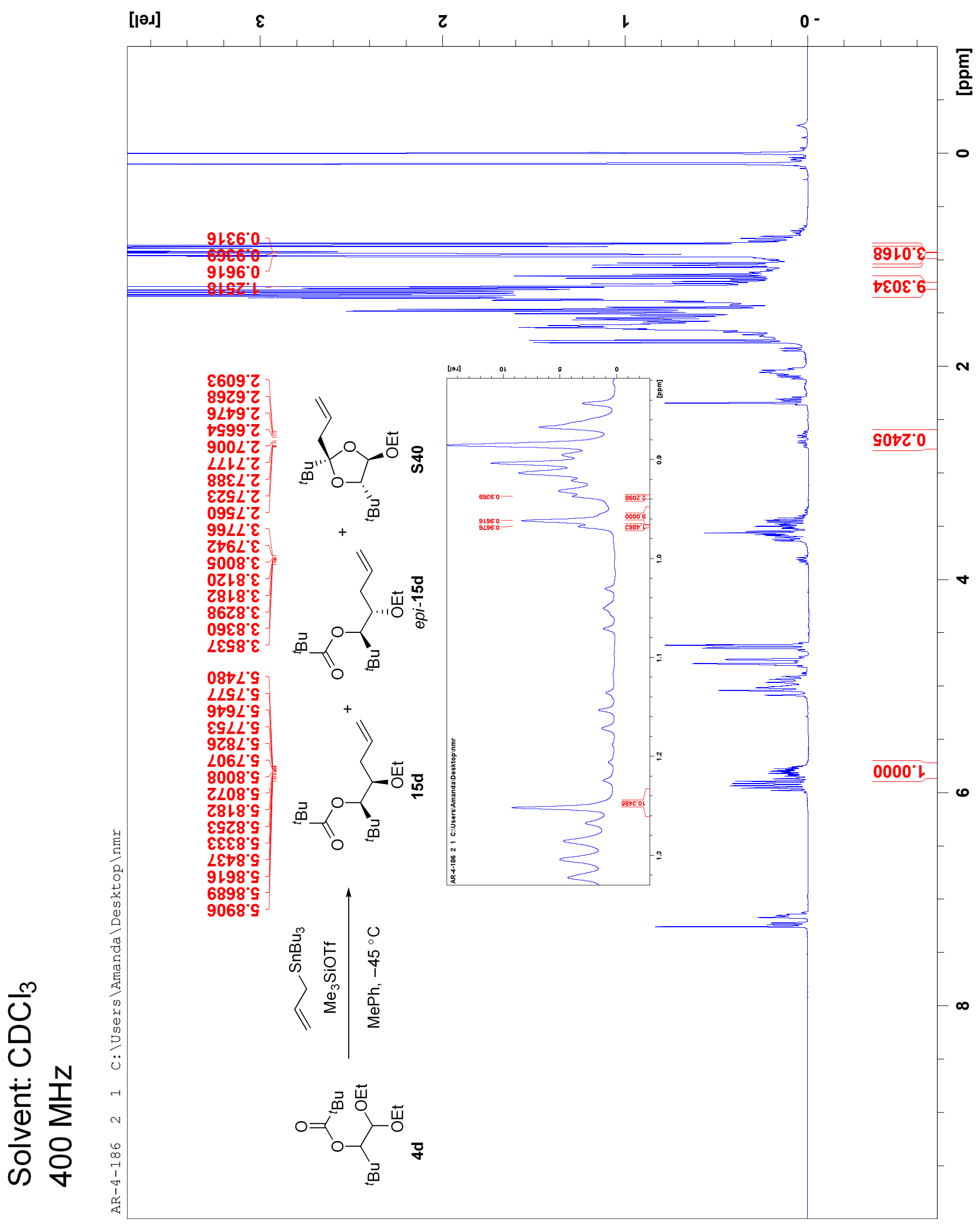




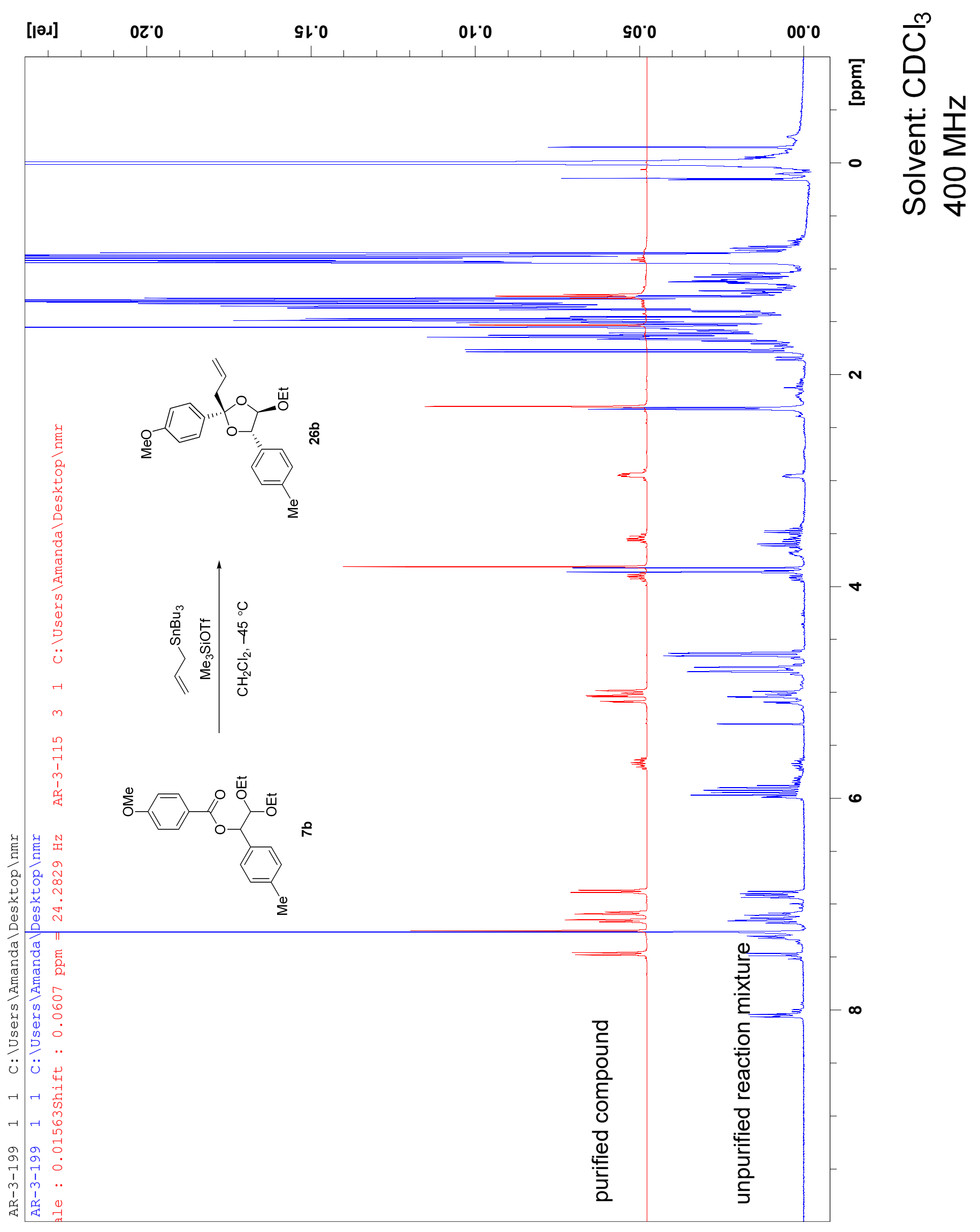




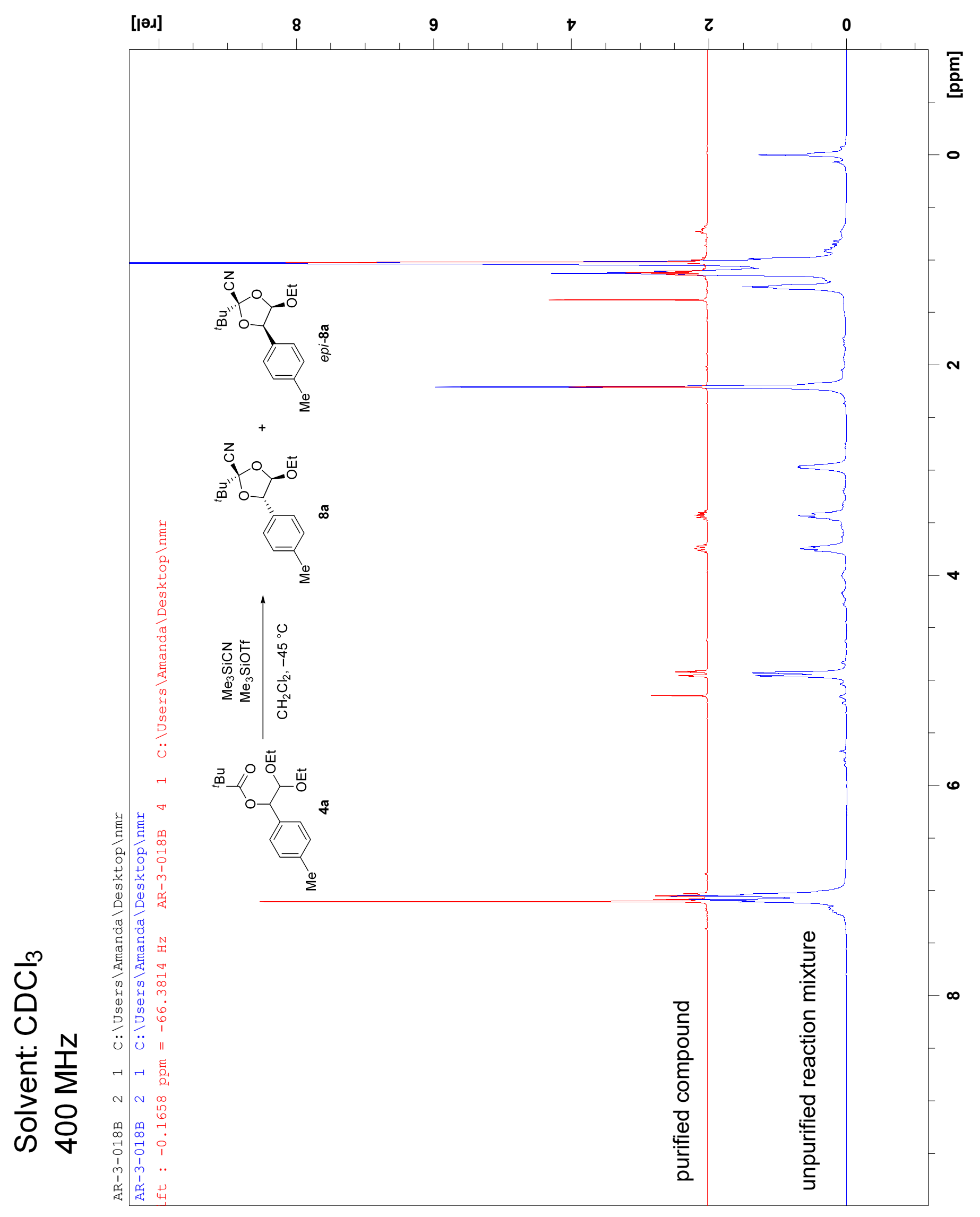




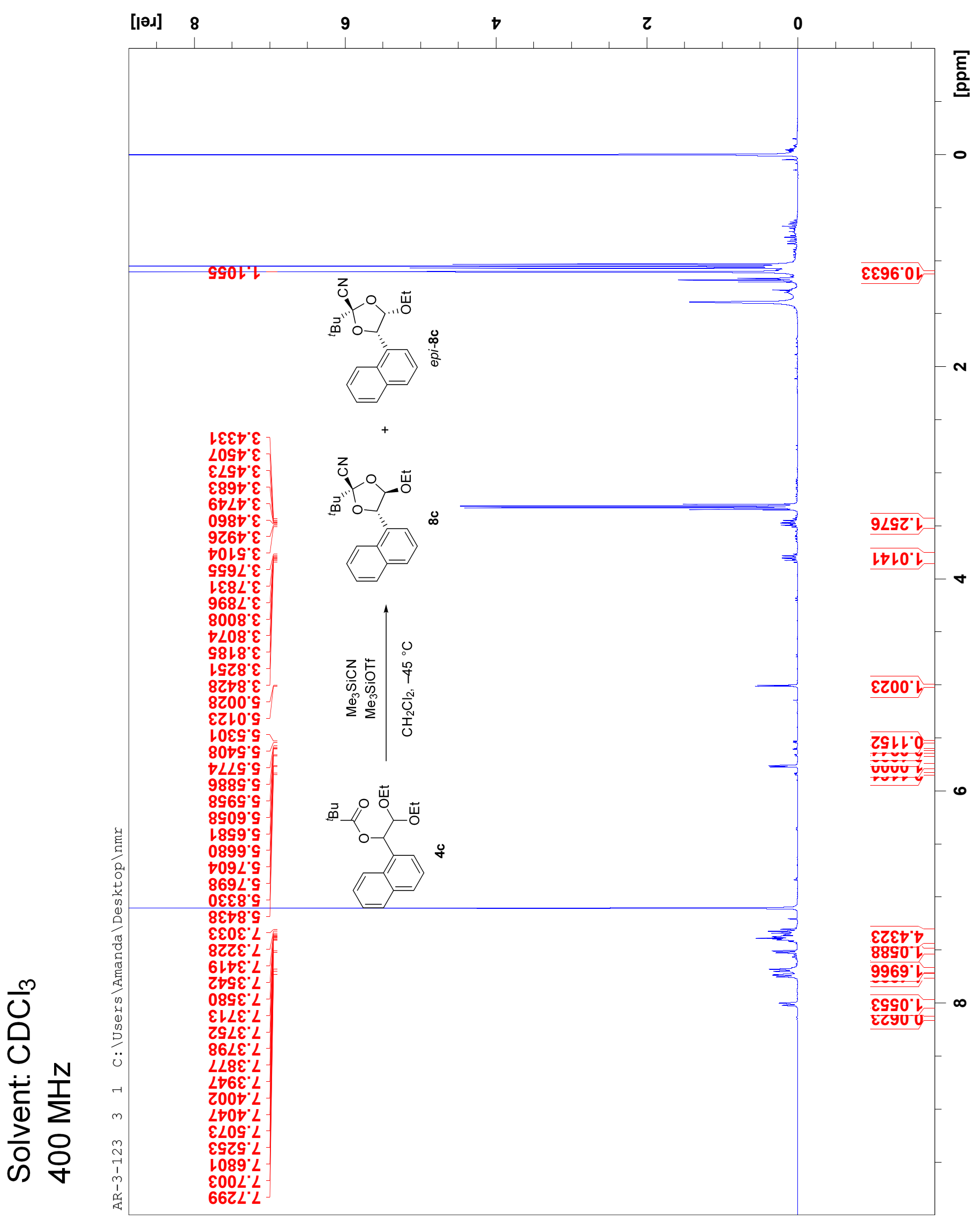




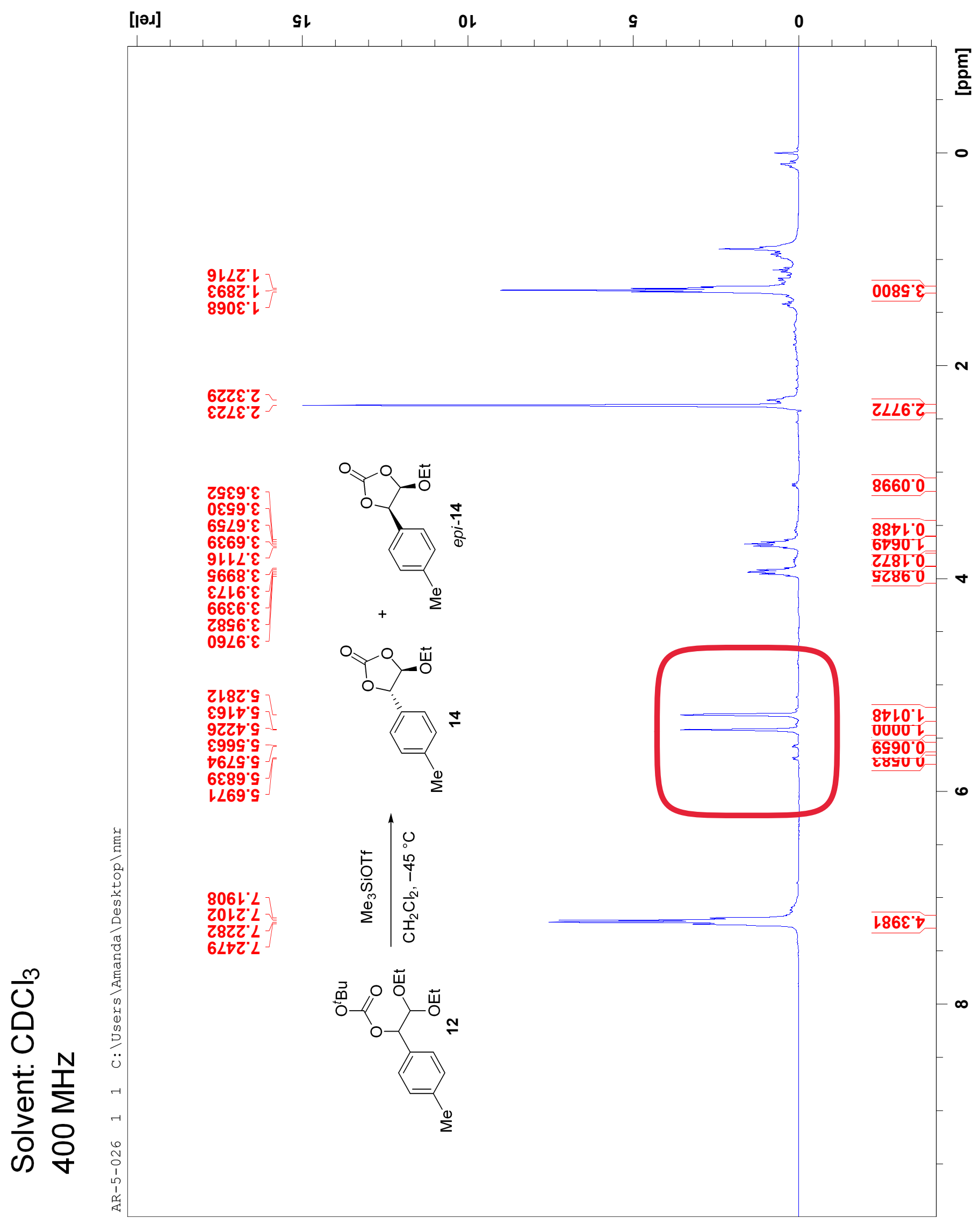




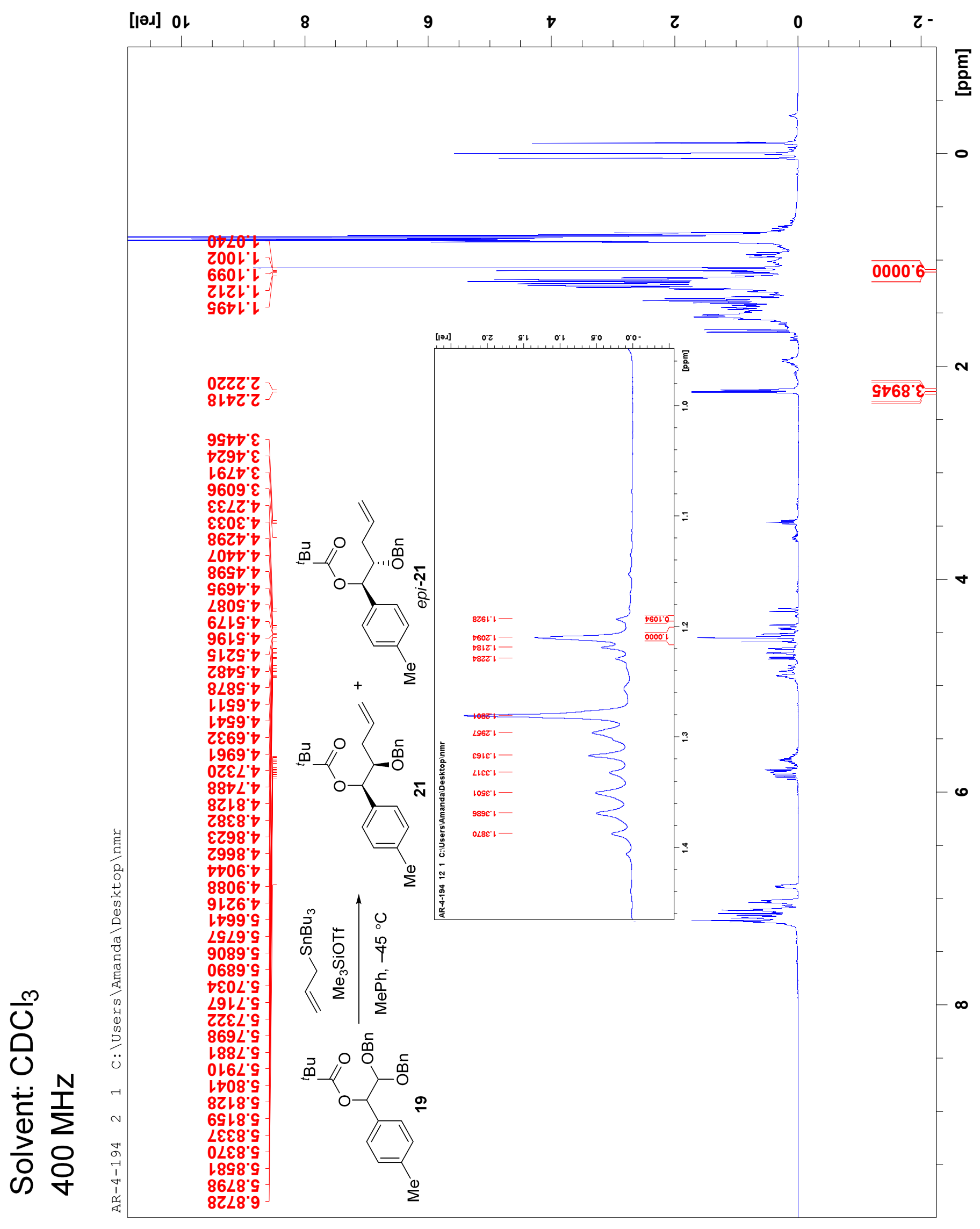

